\title{
ENDF/B-V, LIB-V, and the CSEWG Benchmarks
}

\author{
R. B. Kidman
}

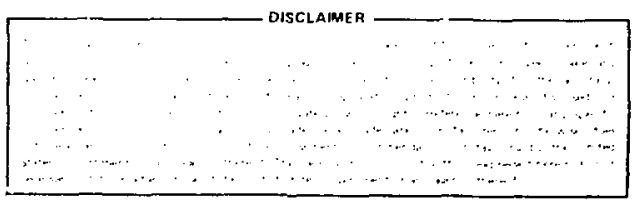

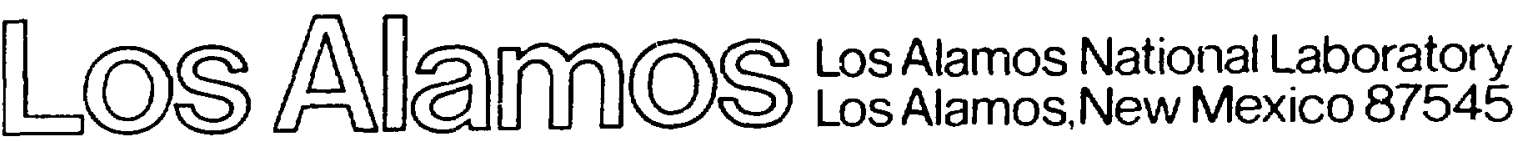


ENDF/B-V, LIB-V, AND THE CSEWG BENCHMARKS

by

R. B. Kidman

\section{ABSTRACT}

A 70-group 1ibrary, LIB-V, generated with the NJOY processing code from ENDF/B-V, is tested on most of the Cross Section Evaluation Working Group (CSEWG) fast reactor benchmarks. Every experimental measurement reported in the benchmark specifications is compared to both diffusion theory and transport theory calculations. Several comparisons with prior benchmark calculations atterpt to assess the effects of data and code improvements.

\section{INTRODUCTION}

Periodically, accumulated reactor physics improvements need to be tested on standard nroblems (benchmarks) to mark progress and establish a set of results that can be referred to in the future.

This report documents the use of 17 benchmarks to test the combined effects of several improvements: ENDF/B-V data, ${ }^{2}$ NJOY ${ }^{3}$ processing, and SUPERX, ONETRAN, ${ }^{4}$ and SUPERB computations. This report can be considered a sequel to an earlier report ${ }^{5}$ in which calculations were based on ENDF/B-IV data, ${ }^{2}$ MINX $^{6}$ preparation, and IDX, 7 ONETRAN, and PERTV ${ }^{8}$ computations .

ENDF/B-V is, of course, a more recent version of evaluated nuclear data than is ENDF/B-IV. NNOY is a more comprehensive, updated version of the MINX processing system. SUPERX and SUPERB are unpublished, overhauled versions of $1 D X$ and PERTV, respectively, that incorporate the following changes.

1. Improved $f$-factor interpoiation

2. Improved elastic downscatter iteration

3. Elastic scattering transfer matrices

4. Elastic downscatter f-factors

5. A new transport cross section computation

6. Isotope and region-dependent fission source matrices 
To study or compare results of this document, Refs. 1 and 5 would be helpful.

II. PROCEDURE

The NJOY code was used to generate 70-group cross sections in the CCCC-IV ${ }^{9}$ format for 93 of the materials in the ENDF/B-V data tapes. The LINX 10 code was then used to form a composite 93-isotope CCCC-IV library from the separate materials. This library is called LIB-V ${ }^{1}$ and its documentation contains the detalls of its generation. The group structure is shown in many of the tables of Appendix B. LIB-V was processed with the weight function (10g-log interpolation between points) shown in Table I. The weight function is intended to model the spectra encountered in fast-reactor benchmark testing and the $14-M e V$ fusion peak. The resulting group-wise function is also shown in Table I.

The computer code $\mathrm{CINX}^{12}$ (modified to pass along elastic removal f-factors, elastic scattering matrices, and isotope chi matrices) was then used to put LIB- $V$ into the format required by SUPERX. The elastic and inelastic transfer cross sections from CINX were arbitrarily limited to 32 downseattering terms (32 includes the in-group term). Composition- and temperature-dependent effective cross sections for each benchmark were ther generated by using SUPERX.

Finally, effective cross sections, fluxes, and adjoints from SUPERX were fed Into SUPERB for the calculation of diffusion theory material worths. The effective cross sections from SUPERX were also fed into ONETRAN to produce $P_{1 / 2}, S_{16}$ transport theory fluxes and adjoints that in turn were fed into SUPERis for the calculation of transport theory material worths. The group velocities and ENDF/B-V delayed neutron data used by SUPERB are presented in Appendix A.

This description of procedures and data should enable one to reproduce any of the results reported here.

\section{RESULTS}

All of the computed results and comparisons with every experimental measurement are compiled in Appendix B. This compilation contains some additional tratisport theory results not included with the previous calculations. 5 The 70group central flux, edge flux, central adjoint, and edge adjoint are shown. The $70 \times 70$ region-dependent chi matrices have been appropriately collapsed (weighted by volume, flux, and neutron production) to 70-group vector chi's for the reactor and each region. These vector chi's allow a better understanding of the 
TABLE I

LIB-V WEIGHTING FUNCTION

Definition

\begin{tabular}{|c|c|c|c|c|c|}
\hline Point & Energy (ev) & Weight & Point & Energy (eV) & Weight \\
\hline 1 & $1.0000 \mathrm{E}-5$ & $0.000 \mathrm{E}+0$ & 13 & $1.0000 \mathrm{E}+7$ & $6.480 \mathrm{E}-1$ \\
\hline 2 & $2.6126 E-3$ & $1.320 \mathrm{E}-10$ & 14 & $1.2790 \mathrm{E}+7$ & $1.360 \mathrm{E}-1$ \\
\hline 3 & $8.7642 \mathrm{E}-1$ & $2.670 E-8$ & 15 & $1.2900 \mathrm{E}+7$ & $1.420 \mathrm{E}-1$ \\
\hline 4 & $7.8893 \mathrm{E}+1$ & $1.940 \mathrm{E}-2$ & 16 & $1.3550 \mathrm{E}+7$ & $1.509 \mathrm{E}+0$ \\
\hline 5 & $3.1203 \mathrm{E}+2$ & $4.490 \mathrm{E}-1$ & 17 & $1.3750 \mathrm{E}+7$ & $2.742 \mathrm{E}+0$ \\
\hline 6 & $1.7956 \mathrm{E}+3$ & $4.802 E+0$ & 18 & $1.3950 \mathrm{E}+7$ & $3.782 \mathrm{E}+0$ \\
\hline 7 & $8.0473 E+3$ & $1.040 \mathrm{E}+1$ & 19 & $1.4070 \mathrm{E}+7$ & $4.008 E+0$ \\
\hline 8 & $4.6309 \mathrm{E}+4$ & $2.030 \mathrm{E}+1$ & 20 & $1.4190 \mathrm{E}+7$ & $3.8395+0$ \\
\hline 9 & $1.6163 E+5$ & $2.290 \mathrm{E}+1$ & 21 & $1.4390 E+7$ & $2.867 \mathrm{E}+0$ \\
\hline 10 & $6.3928 E+5$ & $1.260 \mathrm{E}+1$ & 22 & $1.4590 \mathrm{E}+7$ & $1.637 \mathrm{E}+0$ \\
\hline 11 & $2.8650 \mathrm{E}+6$ & $2.470 \mathrm{E}+0$ & 23 & $1.5550 \mathrm{E}+7$ & $2.650 E-2$ \\
\hline 12 & $4.7237 E+6$ & $9.110 \mathrm{E}-1$ & 24 & $2.0000 \mathrm{E}+7$ & $1.450 \mathrm{E}-3$ \\
\hline
\end{tabular}

Group-Wise Results

\begin{tabular}{rlllll} 
Group & Weight & Group & Weight & Group & Weight \\
\cline { 2 - 4 } 1 & $1.8 E 5 \mathrm{E}+4$ & 25 & $9.118 \mathrm{E}+5$ & 49 & $3.059 \mathrm{E}+3$ \\
2 & $3.927 \mathrm{E}+6$ & 26 & $7.057 \mathrm{E}+5$ & 50 & $2.531 \mathrm{E}+3$ \\
3 & $9.317 \mathrm{E}+5$ & 27 & $5.365 \mathrm{E}+5$ & 51 & $2.094 \mathrm{E}+3$ \\
4 & $1.514 \mathrm{E}+6$ & 28 & $4.079 \mathrm{E}+5$ & 52 & $1.733 \mathrm{E}+3$ \\
5 & $1.321 \mathrm{E}+6$ & 29 & $3.101 \mathrm{E}+5$ & 53 & $1.434 \mathrm{E}+3$ \\
6 & $1.153 \mathrm{E}+6$ & 30 & $2.338 \mathrm{E}+5$ & 54 & $1.187 \mathrm{E}+3$ \\
7 & $1.222 \mathrm{E}+6$ & 31 & $1.671 \mathrm{E}+5$ & 55 & $9.337 \mathrm{E}+2$ \\
8 & $1.566 \mathrm{E}+6$ & 32 & $6.424 \mathrm{E}+4$ & 56 & $6.957 \mathrm{E}+2$ \\
9 & $1.788 \mathrm{E}+6$ & 33 & $5.403 \mathrm{E}+4$ & 57 & $5.183 \mathrm{E}+2$ \\
10 & $1.828 \mathrm{E}+6$ & 34 & $4.544 \mathrm{E}+4$ & 58 & $3.862 \mathrm{E}+2$ \\
11 & $1.868 \mathrm{E}+6$ & 35 & $3.825 \mathrm{E}+4$ & 59 & $2.878 \mathrm{E}+2$ \\
12 & $9.489 \mathrm{E}+5$ & 36 & $3.219 \mathrm{E}+4$ & 60 & $3.741 \mathrm{E}+2$ \\
13 & $9.592 \mathrm{E}+5$ & 37 & $2.708 \mathrm{E}+4$ & 61 & $2.077 \mathrm{E}+2$ \\
14 & $9.696 \mathrm{E}+5$ & 38 & $2.77 \mathrm{E}+4$ & 62 & $1.153 \mathrm{E}+2$ \\
15 & $9.802 \mathrm{E}+5$ & 39 & $1.916 \mathrm{E}+4$ & 63 & $6.401 \mathrm{E}+1$ \\
16 & $9.909 \mathrm{E}+5$ & 40 & $1.612 \mathrm{E}+4$ & 64 & $3.472 \mathrm{E}+1$ \\
17 & $1.002 \mathrm{E}+6$ & 41 & $1.357 \mathrm{E}+4$ & 65 & $1.584 \mathrm{E}+1$ \\
18 & $9.721 \mathrm{E}+5$ & 42 & $1.141 \mathrm{E}+4$ & 66 & $6.970 \mathrm{E}+0$ \\
19 & $9.061 \mathrm{E}+5$ & 43 & $9.516 \mathrm{E}+3$ & 67 & $3.066 \mathrm{E}+0$ \\
20 & $8.440 \mathrm{E}+5$ & 44 & $7.883 \mathrm{E}+3$ & 68 & $1.349 \mathrm{E}+0$ \\
21 & $7.864 \mathrm{E}+5$ & 45 & $6.524 \mathrm{E}+3$ & 69 & $8.352 \mathrm{E}-1$ \\
22 & $1.416 \mathrm{E}+6$ & 46 & $5.398 \mathrm{E}+3$ & 70 & $1.407 \mathrm{E}-1$ \\
23 & $1.231 \mathrm{E}+6$ & 47 & $4.465 \mathrm{E}+3$ & & \\
24 & $1.069 \mathrm{E}+6$ & 48 & $3.697 \mathrm{E}+3$ & &
\end{tabular}


effect of the matrix chi Improvement. As an aid to comparison of ha" c-lethargy width group results to this report, the flux, adjoint, and chi data are also presented in a collapsed 25-group structure. As a further aid to intercomparison, centrally averaged 1-group cross sections are presented for the fission, capture, fission neutrons, and transport reactions of each material.

It is not feasible to show how every data and code change affects the reactor parameters of every critical assembly. What is more amenable to compilation is to lump many changes together and show their combined effect on a few parameters. Therefore, in an effort to separate data associated changes from physics code associated changes, all of the ENDF/B and processing code changes have been lumped together to form the "data" changes, and all of the reactor physics code changes have been Iumped together to form the "code" changes.

Table II compares the present and past ${ }^{5}$ uncorrected diffusion theory eigenvalues and also displays the relative effects of the data and code changes. (Although transport theory results are probably more valid, diffusion theory results must be used to separate the data and code effects because intermediate results were obtained only with diffusion theory.) The eigenvalue effects of the code changes are generally smaller than the eigenvalue effects of the data changes, and the Pu-fueled assemblies generally exhibit larjer data effects than do the U-fueled assemblies. VERA-11A, VERA-1B and ZEBRA-2 stand out as exceptions to these trends. Table III compares the present and past, ${ }^{5}$ uncorrected and corrected transport theory eigenvalues. Only 6 out of 16 of the present corrected eigenvalues are improvements over the past corrected eigenvalues. Most of the improvements are limited to the $U$-fueled assemblies, whereas practically every Pu-fueled assembly is worse.

Tables IV through XI compare present and past transport theory results for several central reaction rate ratios and several central reactivity worths. A1so shown are the relative effects on these central parameters of the data and code changes. (The relative effects do not precisely progress from the past to the present transport theory results because the relative effects were determined using diffusion theory.) Only about $40 \%$ of the present central parameters are improvements over the past. However, thers werz some hot spots of improvement: the $239_{\mathrm{Pu}}$ to $235 \mathrm{U}$ central fission ratio, the $235_{\mathrm{U}}$ and $238_{\mathrm{U}}$ central worths, and $2 P R-3-56 B, Z P R-6-7$ and ZPR-6-6A. 
TABLE II

PAST AND PRESENT UNCORRECTED DIFFUSION THEORY EIGENVALUES

Assembly

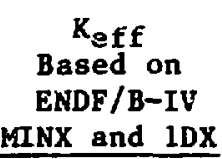

Changes due to ENDF/B-V and NJOY
Changes

due to SUPERX
$K_{\text {eff }}$

Based on ENDF / B-V NJOY and SUPERX

Pu Fueled

\section{JEZEBEL \\ VERA-11A \\ ZEBRA-3 \\ SNEAK-7A \\ SNEAK-7B \\ ZPR-3-48 \\ ZPR-3-56B \\ 2PR-9-31 \\ ZPR-6-7}

ZPPR-2

U Fueled

GODIVA

ZPR-3-6F

VERA-1B

ZPR-3-12

ZPR-3-11

ZE BRA-2

2PR-6-6A

\subsection{5}

0.9449

0.9946

0.9863

0.9892

0.9758

0.9557

0.9708

0.9729

0.9744

0.9976

0.9741

0.9976

1.0089

0.9908

0.9834
$0.0: 30$

0.0094

0.0091

0.0115

0.0162

0.0104

0.0502

0.0127

0.0106

$-0.0058$

$-0.0007$

$-0.0162$

$-0.0008$

0.0026

$-0.0137$

$-0.0026$
$-0.0015$

$-0.0102$

$-0.0078$

$-0.0034$

$-0.0053$

$-0.0033$

$-0.0020$

$-0.0058$

$-0.0011$

$-0.0018$

$-0.0020$

$-0.0054$

0.0126

$-0.0005$

$-0.0053$

0.0185

0.0035
0.9600

0.9441

0.9959

0.9944

1.0001

0.9829

1.0039

0.9908

0.9824

0.9817

0.9666

0.9915

0.9705

0.9963

1.0062

0.9956

0.9843 
TABLE III

PAST AND PRESENT TRANSPORT THEORY EIGENVALUES

\begin{tabular}{|c|c|c|c|c|c|c|}
\hline \multirow{2}{*}{$\begin{array}{l}\text { Critica] } \\
\text { Assembly } \\
\end{array}$} & \multicolumn{2}{|c|}{ Uncorrected $K_{\text {eff }}$} & \multirow[b]{2}{*}{ Correction $^{a}$} & \multicolumn{2}{|c|}{ Corrected $K_{\text {eff }}$} & \multirow{2}{*}{$\begin{array}{l}\text { Present } \\
- \text { Past } \\
\end{array}$} \\
\hline & Past & Present & & Past & Present & \\
\hline \multicolumn{7}{|l|}{ Pu Fueled } \\
\hline JEZEBEL & 0.9998 & 1.0111 & $-0.0053^{b}$ & 0.9945 & 1.0058 & 0.0113 \\
\hline VERA-1 IA & 0.9923 & 0.9884 & & 0.9923 & 0.9884 & -0.0039 \\
\hline ZEBRA-3 & 1.0080 & 1.0090 & & 1.0080 & 1.0090 & 0.0010 \\
\hline SNEAK-7A & 1.0016 & 1.0056 & & 1.0016 & 1.0056 & 0.0040 \\
\hline SNEAK-7B & 0.9968 & 1.0048 & & 0.9968 & 1.0048 & 0.0080 \\
\hline$Z P R-3-48$ & 0.9850 & 0.9883 & 0.0183 & 1.0033 & 1.0066 & 0.0033 \\
\hline$Z P R-3-56 B$ & 1.0086 & 1.0106 & $-0.0046^{c}$ & 1.0040 & 1.0060 & 0.0020 \\
\hline $2 P R-9-31$ & & 0.9955 & 0.0128 & & 1.0083 & \\
\hline $2 P R-6-7$ & 0.9762 & 0.9841 & 0.0166 & 0.9928 & 1.0007 & 0.0079 \\
\hline ZPPR-2 & 0.9967 & 1.0017 & 0.0175 & 1.0142 & 1.0192 & 0.0050 \\
\hline \multicolumn{7}{|l|}{ U Fueled } \\
\hline GODIVA & 1.0110 & 1.0028 & $-0.0047^{b}$ & 1.0063 & $0.998 i$ & -0.0082 \\
\hline$Z P R-3-6 F$ & 1.0178 & 1.0101 & & 1.0178 & 1.0101 & -0.0077 \\
\hline VERA-1 B & 0.9994 & 0.9923 & & 0.9994 & 0.9923 & -0.0071 \\
\hline $2 P R-3-12$ & 1.0090 & 1.0061 & & 1.0090 & 1.0061 & -0.0029 \\
\hline $2 P R-3-11$ & 1.0153 & 1.0121 & & 1.0153 & 1.0121 & -0.0032 \\
\hline ZEBRA-2 & 0.9963 & 0.9988 & & 0.9963 & 0.9988 & 0.0025 \\
\hline $2 P R-6-6 A$ & 0.9883 & 0.9854 & 0.0073 & 0.9956 & 0.9927 & -0.0029 \\
\hline
\end{tabular}

af no correction factor is listed, then the specifications simply do not give one, or the specified model was already adjusted to account for heterogeneities.

$\mathrm{b}_{\text {This is }}: \mathrm{i} \%$ Los Alamos result for $\mathrm{S}_{16} \rightarrow \mathrm{S}_{\infty}$ corrections as reported in Ref. 5.

${ }^{C}$ The $1 D+2 D$ correction from Ref. 5 was added to the heterogeneity correction of Ref. 1 because the I-D ZPR-3-56B model is not an equivalent K-1.0 model. 
TABLE IV

PAST AND PRESENT 239 Pu TO 235 CENTRAL FISSION RATIOS (C/E)

$$
\sigma_{\mathrm{f}}^{239} / \sigma_{\mathrm{f}}^{235}
$$

Critical

Assembly

Based on ENDF/B-IV, MINX, IDX, ONETRAN
Changes due to ENDF/B-V and NJOY

$$
\sigma_{\mathrm{f}}^{239} / \sigma_{\mathrm{f}}^{235}
$$

Changes

due to

SUPERX
Based on ENDF/B-V, NJOY, SUPERX, ONETRAN

\section{Pu Fueled}

\begin{tabular}{lllrl} 
JEZEBEL & 0.9364 & 0.0349 & 0.0006 & 0.9724 \\
YERA-11A & 1.0836 & 0.0442 & -0.0263 & 1.1035 \\
ZEBRA-3 & 0.9913 & 0.0176 & -0.0003 & 1.0092 \\
SNEAK-7A & 0.9606 & 0.0540 & -0.0328 & 0.9835 \\
SNEAK-7B & 0.9861 & 0.0385 & -0.0155 & 1.0098 \\
ZPR-3-56B & 0.9382 & 0.0298 & -0.0114 & 0.9656 \\
ZPR-9-31 & & & -0.0184 & 1.0630 \\
ZPR-6-7 & 0.9612 & 0.0307 & -0.0085 & 0.9845 \\
ZPPR-2 & 0.9771 & 0.0305 & -0.0087 & 0.9999 \\
& & & & \\
U Fueled & & & & \\
\hline & & & & \\
GODIVA & 0.9728 & 0.0203 & 0.0010 & 1.0343 \\
ZPR-3-6F & 1.0229 & 0.0151 & 0.0002 & 1.0813 \\
VERA-1B & 1.0648 & 0.0481 & -0.0323 & 1.0129 \\
ZPR-3-12 & 0.9969 & 0.0330 & -0.0177 & 1.0007 \\
ZPR-3-11 & 0.9843 & 0.0160 & 0.0002 & 1.0157 \\
ZEBRA-2 & 0.9941 & 0.0558 & -0.0344 &
\end{tabular}


TABLE V

PAST AND PRESENT $238_{\mathrm{U}}$ TO $235_{\mathrm{U}}$ CENTRAL FISSION RATIOS (C/E)

\begin{tabular}{|c|c|c|c|c|}
\hline & $\sigma_{f}^{238 / \sigma_{f}^{235}}$ & & & $\sigma_{f}^{238} / \sigma_{f}^{235}$ \\
\hline $\begin{array}{l}\text { Critical } \\
\text { Assembly } \\
\end{array}$ & $\begin{array}{l}\quad \text { Based on } \\
\text { ENDF/B-IV,MINX, } \\
\text { 1DX, ONETRAN }\end{array}$ & $\begin{array}{l}\text { Changes due } \\
\text { to ENDE/B-V } \\
\text { and NJOY } \\
\end{array}$ & $\begin{array}{l}\text { Changes } \\
\text { due to } \\
\text { SUPaRX }\end{array}$ & $\begin{array}{l}\text { Based on } \\
\text { ENDE/B-V, NJOY, } \\
\text { SUPERX, ONETRAN }\end{array}$ \\
\hline
\end{tabular}

Pu Fueled

$\begin{array}{lllll}\text { JEZEBEL } & 0.9218 & 0.0017 & -0.0063 & 0.9169 \\ \text { VERA-11A } & 1.1532 & 0.0696 & -0.0372 & 1.1916 \\ \text { ZEBRA-3 } & 1.0062 & 0.0261 & -0.0044 & 1.0293 \\ \text { SNEAK-7A } & 0.9255 & 0.0974 & -0.0523 & 0.9767 \\ \text { SNEAK-7B } & 0.9803 & 0.0692 & -0.0194 & 1.0341 \\ \text { ZPR-3-48 } & 1.0350 & 0.0818 & -0.0520 & 1.0714 \\ \text { ZPR-3-56B } & 0.8672 & 0.1077 & -0.0209 & 0.9972 \\ \text { ZPR-9-31 } & & & -0.0323 & 1.0576 \\ \text { ZPR-6-7 } & 0.9315 & 0.0697 & -0.0134 & 0.9934 \\ \text { ZPPR-2 } & 1.0528 & 0.0703 & -0.0173 & 1.1102\end{array}$

U Fueled

$\begin{array}{lrrrr}\text { GODIVA } & 1.0526 & -r .0177 & 0.0024 & 1.0365 \\ \text { ZPR-3-6F } & 1.0172 & 0.0093 & 0.0033 & 1.0342 \\ \text { VERA-1B } & 1.2147 & 0.0768 & -0.0613 & 1.2331 \\ \text { ZPR-3-12 } & 1.0691 & 0.0511 & -0.0259 & 1.0917 \\ \text { ZPR-3-11 } & 1.0562 & 0.0175 & -0.0003 & 1.0745 \\ \text { ZEBRA-2 } & 1.0436 & 0.0800 & -0.0660 & 1.06 C 3 \\ \text { ZPR-6-6A } & 0.9420 & 0.0401 & -0.0007 & 0.9855\end{array}$




\section{TABLE VI}

PAST AND PRESENT $238_{\mathrm{U}}$ CAPTURE TO $235 \mathrm{U}$ FISSION CENTRAL RATIOS (C/E)

\begin{tabular}{|c|c|c|c|c|}
\hline & $\sigma_{f}^{238} / \sigma_{f}^{235}$ & & & $\sigma_{f}^{258} / \sigma_{f}^{235}$ \\
\hline $\begin{array}{l}\text { Critlcal } \\
\text { Assenbly }\end{array}$ & $\begin{array}{l}\text { Based on } \\
\text { ENDF/B-IV,MINX, } \\
\text { 1DX, ONETRAN }\end{array}$ & $\begin{array}{l}\text { Changes due } \\
\text { to ENDF/B-V } \\
\text { and NJOY }\end{array}$ & $\begin{array}{l}\text { Changes } \\
\text { due to } \\
\text { SUPERX }\end{array}$ & $\begin{array}{l}\text { Based on } \\
\text { ENDF/B-V, NJOY, } \\
\text { SUPERX, ONETRAN }\end{array}$ \\
\hline
\end{tabular}

Pu Fueled

$\begin{array}{llrll}\text { SNEAK-7A } & 0.9962 & 0.0066 & -0.0109 & 0.9914 \\ \text { SNEAK-7B } & 1.0395 & -0.0039 & -0.0072 & 1.0279 \\ \text { ZPR-3-48 } & 0.9768 & 0.0137 & -0.0158 & 0.9733 \\ \text { ZPK-9-31 } & & & -0.0116 & 1.1001 \\ \text { ZPR-6-7 } & 1.0542 & 0.0168 & -0.0132 & 1.0569\end{array}$

U Fueled

$\begin{array}{llrrr}\text { ZPR-3-6F } & 0.9344 & -0.0087 & 0.0017 & 0.9258 \\ \text { VERA-1B } & 0.9468 & -0.0505 & 0.0484 & 0.9438 \\ \text { ZPR-3-12 } & 0.9697 & -0.0122 & 0.0089 & 0.9657 \\ \text { ZPR-3-11 } & 0.9690 & -0.0006 & 0.0015 & 0.9692 \\ \text { ZEBRA-2 } & 0.9697 & 0.0277 & -0.0422 & 0.9548 \\ \text { ZPR-6-6A } & 1.0318 & 0.0138 & -0.0086 & 1.0363\end{array}$


TABLE VII

PAST AND PRESENT 239 Pu CENTRAL WORTHS (C/E)

\begin{tabular}{|c|c|c|c|c|}
\hline & $\begin{array}{l}2{ }^{39} \mathrm{Pu} \text { Worths } \\
\text { Based on } \\
\text { ENDF/B-IV, }\end{array}$ & Changes due & Changes due & $\begin{array}{l}239 \mathrm{Pu} \text { Worths } \\
\text { Based on } \\
\text { ENDF/B-V }\end{array}$ \\
\hline $\begin{array}{l}\text { Critical } \\
\text { Assembly }\end{array}$ & $\begin{array}{l}\text { MINX, IDX, } \\
\text { ONETRAN, PERTV }\end{array}$ & $\begin{array}{l}\text { to ENDF/B-V } \\
\text { and NJOY }\end{array}$ & $\begin{array}{l}\text { to SUPERX, } \\
\text { SUPERB }\end{array}$ & $\begin{array}{l}\text { NJOY, SUPERX, } \\
\text { ONETRAN, SUPER B }\end{array}$ \\
\hline
\end{tabular}

Pu Fueled

$\begin{array}{llrrr}\text { JEZEBEL } & 1.0353 & -0.0143 & -0.0015 & 1.0156 \\ \text { ZEBRA-3 } & 1.1377 & 0.0354 & 0.0024 & 1.1831 \\ \text { SNEAK-7A } & 1.0360 & 0.0261 & -0.0208 & 1.0525 \\ \text { SNEAK-7B } & 1.0851 & 0.0107 & -0.0098 & 1.0877 \\ \text { ZPR-3-48 } & 1.1437 & 0.0435 & -0.0238 & 1.1806 \\ \text { ZPR-3-56B } & 1.3551 & -0.1714 & -0.0189 & 1.1410 \\ \text { ZPR-9-31 } & & & -0.0154 & 1.1607 \\ \text { ZPR-6-7 } & 1.1662 & 0.0229 & -0.0170 & 1.1731 \\ \text { ZPPR-2 } & 1.0784 & 0.0268 & -0.0097 & 1.1005\end{array}$

U Fueled

$\begin{array}{lrrrr}\text { GODIVA } & 1.0021 & -0.0104 & -0.0015 & 0.9990 \\ \text { ZPR-3-6F } & 1.0600 & 0.0244 & 0.0045 & 1.0959 \\ \text { VERA-1B } & 1.0142 & 0.0760 & -0.0632 & 1.0400 \\ \text { ZPR-3-12 } & 0.9725 & 0.0524 & -0.0188 & 1.0161 \\ \text { ZPR-3-11 } & 1.0279 & 0.0392 & 0.0136 & 1.0895 \\ \text { ZEBRA-2 } & 1.0506 & 0.1501 & -0.1127 & 1.1008 \\ \text { ZPR-6-6A } & 1.0674 & 0.0447 & -0.0193 & 1.0943\end{array}$


TABLE VIII

PAST AND PRESENT $235 \mathrm{U}$ CENTRAL WORTHS (C/E)

\begin{tabular}{|c|c|c|c|c|}
\hline & $\begin{array}{l}235_{\mathrm{U}} \text { Worths } \\
\text { Based on } \\
\text { ENDE/B-IV, }\end{array}$ & Changes due & Changes due & $\begin{array}{l}235_{\mathrm{U}} \text { Worths } \\
\text { Based on } \\
\text { ENDF/B-V }\end{array}$ \\
\hline $\begin{array}{l}\text { Critical } \\
\text { Assembly }\end{array}$ & $\begin{array}{l}\text { MINX, 1DX, } \\
\text { ONETRAN, PERTV }\end{array}$ & $\begin{array}{l}\text { to ENDF/B-V } \\
\text { and NJOY }\end{array}$ & $\begin{array}{l}\text { to SUPERX, } \\
\text { SUPERB }\end{array}$ & $\begin{array}{l}\text { NJOY, SUPERX, } \\
\text { ONETRAY, SUPER B }\end{array}$ \\
\hline
\end{tabular}

Pu Fueled

\begin{tabular}{llrrr} 
JEZEBEL & 1.0737 & -0.0295 & -0.0056 & 1.0212 \\
ZEBRA-3 & 1.1457 & 0.0108 & -0.0156 & 1.1464 \\
SNEAK-7A & 1.0389 & -0.0278 & -0.0030 & 1.0176 \\
SNEAK-7B & 1.0597 & -0.0331 & -0.0091 & 1.0185 \\
ZPR-3-48 & 1.1569 & -0.0071 & -0.0122 & 1.1533 \\
ZPR-3-56B & 1.3296 & -0.2222 & -0.0184 & 1.0582 \\
ZPR-9-31 & & & -0.0144 & 1.1413 \\
ZPR-6-7 & 1.1607 & -0.0217 & -0.0227 & 1.1157 \\
ZPPR-2 & 1.1925 & -0.0062 & -0.0169 & 1.1739 \\
& & & & \\
U Fueled & & & & \\
\hline & & & & \\
GODIVA & 1.0079 & -0.0272 & -0.0022 & 0.9798 \\
ZPR-3-6F & 0.8701 & 0.0045 & 0.0007 & 0.8800 \\
VEIA-1B & 0.9948 & 0.0491 & -0.0485 & 1.0074 \\
ZPR-3-12 & 0.9733 & 0.0217 & -0.0162 & 0.9885 \\
ZPR-3-11 & 1.0822 & 0.0219 & -0.0028 & 1.1103 \\
ZEBRA-2 & 1.0808 & 0.1673 & -0.0962 & 1.0926 \\
ZPR-6-6A & 1.1222 & 0.0179 & -0.0223 & 1.1191
\end{tabular}


TABLE IX

PAST AND PRESENT 238 CENTRAL WORTHS (C/E)

\begin{tabular}{|c|c|c|c|c|}
\hline & $\begin{array}{l}238_{\mathrm{y}} \text { Worths } \\
\text { Based on } \\
\text { ENDF/B-IV, }\end{array}$ & Changes due & Changes due & $\begin{array}{l}23 B_{U} \text { Worths } \\
\text { Based on } \\
\text { ENDF/B-V }\end{array}$ \\
\hline $\begin{array}{l}\text { Critical } \\
\text { Assembly }\end{array}$ & $\begin{array}{l}\text { MINX, 1DX, } \\
\text { ONETRAN, PERTV }\end{array}$ & $\begin{array}{l}\text { to ENDF/B-V } \\
\text { and NJOY }\end{array}$ & $\begin{array}{l}\text { to SUPERX, } \\
\text { SUPERB } \\
\end{array}$ & $\begin{array}{l}\text { NJOY, SUPERX, } \\
\text { ONETRAN, SUPERB }\end{array}$ \\
\hline
\end{tabular}

Pu Fueled

JEZEBEL

ZEBRA-3

SNEAK-7A

SNEAK-7B

ZPR-3-48

ZPR-3-56B

$2 P_{K}-9-31$

2PR-6-7

0.9826

0.0104

$-0.0264$

0.9437

0.0253

$-0.0021$

1.0785

1.1315

$-0.1270$

0.0962

1.0898

1.0510

0.0213

1.0361

1.1550

0.0700

1.1643

1.3969

0.0274

1.1487

1.0415

0.0261

1.0308

0.0191

1.0388

U Fueled

GODIVA

ZPR-3-6F

VERA-1B

ZPR $-3-12$

ZPR-3-11

ZF,BRA-2

2PR-6-6A
0.9857

2.7361

1.3545

0.9534

1.0011

1.0451

1.1897
0.0047

0.1870

0.7632

$-0.0931$

$-0.0081$

$-0.0657$

$-0.0188$
$-0.0038$

0.0519

$-0.6123$

0.0750

$-0.0066$

0.0517

0.0098
1.0028

2.9267

1.5535

0.9337

i. 0096

1.0332

1.1739 
TABLE $X$

PAST AND PRESENT $10^{1}$ B CENTRAL WORTHS (C/E)

$\begin{array}{cc} & 10 \mathrm{~B} \text { Worths } \\ & \text { Based on } \\ & \text { ENDF/B-IV, } \\ \text { Critical } & \text { MINX, 10X, } \\ \text { Assembly } & \text { ONETRAN,PERTV }\end{array}$

Pu Fueled
0.9800

0.9818

0.9253

0.9124

1.0492

1.0227

1.1334

0.9649

2PR-6-7

ZPR-2

\section{U Fueled}

GODIVA

ZPR-3-6F

VERA-1B

ZPR-3-11

ZEBRA-2

ZPR-6-6A
0.8685
0.9973
1.0469
0.9438
0.8529
0.9535

$-0.0238$

$-0.0228$

$-0.1373$

$-0.0723$

$-0.1181$

$-0.0853$

$-0.0700$

$-0.0550$

$-0.0376$

$-0.0177$

$-0.1516$

$-0.0167$

$-0.1438$

$-0.0365$
10 Borths Based on ENDF / B- V V Changes due to SUPERX, SUPERB ONETRAN, SUPER B

$\begin{array}{ll}0.0311 & 0.9988 \\ 0.0083 & 0.9678 \\ 0.1255 & 0.9086 \\ 0.0641 & 0.8962 \\ 0.1299 & 1.0602 \\ 0.0486 & 0.8967 \\ 0.0838 & 1.0287 \\ 0.0639 & 1.1175 \\ 0.0595 & 0.9648\end{array}$

0.0053

0.8345

0.9659

1.0298

0.9308

0.8730

0.9436 


\section{TABLE XI}

PAST AND PRESENT 23 Na CENTRAL WORTHS (C/E)

\begin{tabular}{cc} 
& 23 Na Worths \\
& Based on \\
& ENDF/B-IV, \\
Critical & MINX, 1DX, \\
Assembly & ONETRAN,PERTV \\
\hline
\end{tabular}

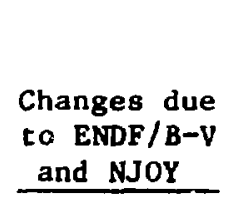

$23 \mathrm{Na}$ Worths Based on ENDF / B-V NJOY, SUPERX, ONETRAN, SUPERB

\section{Pu Fueled}

\section{ZEBRA-3 \\ ZPR-3-48 \\ ZPR-3-56B \\ 2PR-9-31 \\ 2PR-6-7 \\ ZPPR -2}

U Fueled

$$
\begin{aligned}
& 1.3487 \\
& 2.4266 \\
& 3.5395 \\
& 1.2483 \\
& 1.2389
\end{aligned}
$$

0.2746

0.3612

2.0580

2.5187

$-3.8754$

$$
\begin{array}{r}
0.0885 \\
1.0028 \\
-0.4607 \\
0.1832
\end{array}
$$

0.1671

0.0300

$-0.0065$

0.1373

$-1.9958$

$-0.0297$

$$
\begin{aligned}
& -0.0564 \\
& -1.0345 \\
& -0.3131 \\
& -0.2169 \\
& -0.1752 \\
& -0.1566
\end{aligned}
$$

$$
\begin{aligned}
& 1.4147 \\
& 2.4811 \\
& 2.2878 \\
& 1.3477 \\
& 1.2525 \\
& 1.2593
\end{aligned}
$$

2EBRA-2

2PR-6-6A

$$
\begin{array}{r}
0.0940 \\
-0.0186 \\
-0.1324 \\
2.2097 \\
3.5976
\end{array}
$$

0.3627

0.3372

2.1176

2.6904

$-0.0817$ 
The computer run times for the various code submittals of this study are shown in Table XII. The run times are longer than in the past mainly because 70-groups and fission source matrices are being used.

TABLE XII

COMPUTER RUN TIMES

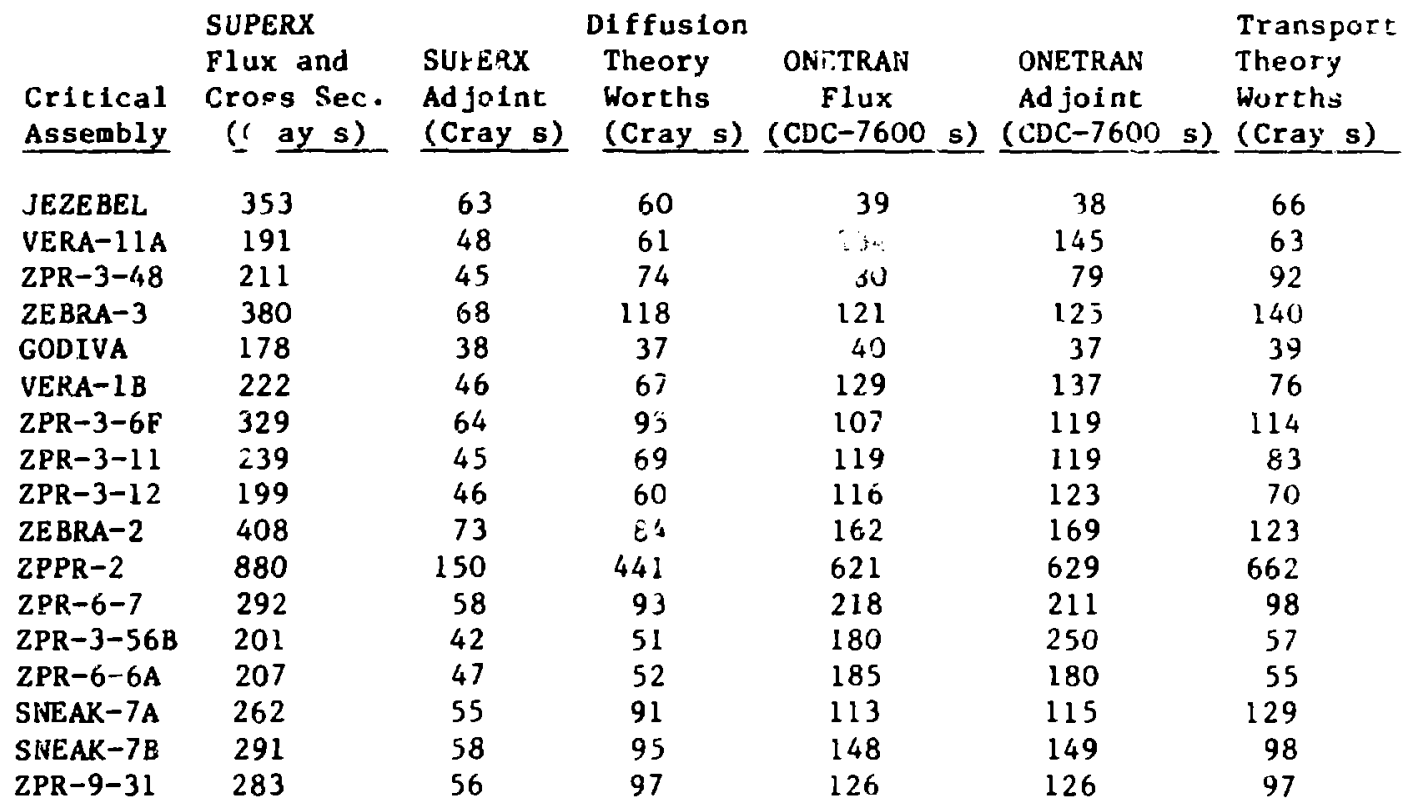

IV. CONCLUSIONS

Considering all of the reactor parameters of all the critical assemblies, present transport theory results represent an improvement over pas transport theory results in only about $40 \%$ of the cases. According to the data and code change effects set forth in Tables II through $X I$, a better improvenent record could perhaps be compiled if the code changes were applied to ENDF/B-IV data. 


\section{REFERENCES}

1. H. Alter, R. B. Kidman, R. LaBauve, R. Protsik, and B. A. Zolator, "ENDF-202 Cross Section Evaluation Working Group Benchmark Specifications," Brookhaven National Laboratory report BNL-19302 (1974).

2. D. Garber, Ed., "Data Formats and Procedures for the ENDF Neutron Cross Section Library," Brookhaven National Laboratory report BNL 50274 (1976).

3. R. E. MacFarlane, R. J. Barrett, D. W. Muir, and R. M. Bolcourt, "The NJOY Nuclear Data Processing System: User's Manual," Los Alamos Scientific Laboratory report LA-7584-M (1978).

4. T. R. Hill, "ONETRAN: A Discrete Ordinates Finite Element Code for the Solution of the One-Dimensional Multigroup Transport Equation," Los Alamos Scientific Laboratory report LA-5990-MS (1975).

5. R. B. Kidman, "ENDF/B-IV, LIB-IV, and the CSEWG Benchmarks," Los Alamos Scientiflc Laboratory report LA-7355-MS (1978).

6. C. R. Weisbin, P. D. Soran, R. E. MacFarlane, D. R. Harris, R. J. LaBauve, J. S. Hendricks, J. E. White, and R. B. Kidman, "MINX, A Multigroup Interpretation of Nuclear X-Sections from ENDF/B," Los Alamos Scietific Laboratory report LA-6486-MS (1976).

7. R. W. Hardie and W. W. Little, JE., "1DX, A One-Dimensional Diffusion Code for Generating Effective Nuclear Cross Sections," Battelle Northwest Laboratory report BNWL-954 (1969).

8. R. W. Hardie and W. W. Little, Jr., "PERT-V, A Two-Dimenstonal Perturbation Code for Fast Reactor Analysis," Battelle Northwest Laboratory report BNWL-1162 (1969).

9. R. Douglas O'De11, "Standard Interface F1les and Procedures for Reactor Physics Codes, Version IV," Los Alamos Scientific Laboratory report LA-6941-MS (1977).

10. R. E. MacFarlane and R. B. KIdman, "LINX and BINX: CCCC Ut1lity Codes for the MINX Multigroup Processing Code," Los Alanos Scientific Laboratory report LA-6219-MS (1976).

11. R. B. Kidman and R. E. MacFarlane, "LIB-V, A Processing of ENDF/B-V Data," Los Alamos Sclentific Laboratory report to be published.

12. R. B. KIdman and R. E. MacFarlane, "CINX, Collapsed Interpretaclon of Nuclear X-Sections," Los Alamos Sclentific Laboratory report LA-6287-MS (1976). 
DELAYED NEUTRON DATA

This appendix contalns the processed delayed neutron data used in this study. The basic source of daca was ENDF/B-V.

Delayed neutron data for $234 \mathrm{U}$ and $236 \mathrm{U}$ was fabricated. The absolute delayed neutron yields are estimates, whereas the rest of the data for $234 \mathrm{U}$ was assumed to be the same as $235 \mathrm{U}$ data, and the rest of the data for $236 \mathrm{U}$ was assumed to be the same as $23 B_{U}$ data.

The delayed fission spectra for $240 \mathrm{Pu}$ and $24 \mathrm{l}^{\mathrm{Pu}}$ are not shown because they are the same as the ${ }^{23} \mathrm{Pu}$ delayed fission spectra. Similarly, ${ }^{23 j} \mathrm{U}$ spectra are not shown because they are the same as $235 \mathrm{U}$ data.

The group velocities, which are used in the calculation of neutron generation times, are shown in Tzbie A-VIII. 
TABLE A-I

ABSOLUTE DELAYED NEUTRON YIELD PER FISSION

\begin{tabular}{ll} 
Isotope & Yleld \\
\hline Th-232 & 0.05270 \\
U-233 & 0.00740 \\
$U-234$ & $0.01000^{a}$ \\
$U-235$ & 0.01670 \\
$U-236$ & $0.02600^{a}$ \\
$U-238$ & 0.04400 \\
Pu-239 & 0.00645 \\
Pu-240 & 0.00900 \\
Pu-241 & 0.01620 \\
\hline
\end{tabular}

Estimates.

TABLE A-II

FRACTIONAL DELAYED NEUTRON YIELD IN PRECURSOR GROUPS

\begin{tabular}{|c|c|c|c|c|c|c|}
\hline \multirow[b]{2}{*}{ Isotope } & \multicolumn{6}{|c|}{ Precursor Groups } \\
\hline & 1 & 2 & 3 & 4 & 5 & 6 \\
\hline $\begin{array}{l}T h=232 \\
U-233 \\
U-235 \\
U-238 \\
P u-239 \\
P u=240 \\
\text { Pu-24I }\end{array}$ & $\begin{array}{l}0.034 \\
0.086 \\
0.038 \\
0.013 \\
0.038 \\
0.028 \\
0.010\end{array}$ & $\begin{array}{l}0.150 \\
0.274 \\
0.213 \\
0.137 \\
0.280 \\
0.273 \\
0.229\end{array}$ & $\begin{array}{l}0.155 \\
0.227 \\
0.188 \\
0.162 \\
0.216 \\
0.192 \\
0.173\end{array}$ & $\begin{array}{l}0.440^{\circ} \\
0.317 \\
0.407 \\
0.388 \\
0.328 \\
0.350 \\
0.390\end{array}$ & $\begin{array}{l}0.172 \\
0.073 \\
0.128 \\
0.225 \\
0.103 \\
0.128 \\
0.182\end{array}$ & $\begin{array}{l}0.043 \\
0.023 \\
0.026 \\
0.075 \\
0.035 \\
0.029 \\
0.016\end{array}$ \\
\hline
\end{tabular}

TABLE A-III

PRECURSOR GROUP DECAY CONSTANTS $\left(s^{-1}\right)$

Precirsor Groups

\begin{tabular}{|c|c|c|c|c|c|c|}
\hline & \multirow{2}{*}{\multicolumn{3}{|c|}{3}} & \multirow[b]{2}{*}{4} & \multirow[b]{2}{*}{5} & \multirow[b]{2}{*}{6} \\
\hline Isotope & & & & & & \\
\hline $\begin{array}{l}\text { Ih-232 } \\
U-233 \\
\delta-235 \\
U-238 \\
P u=239 \\
P u-240 \\
\text { Pu-241 }\end{array}$ & $\begin{array}{l}1.237-2 \\
1.258-2 \\
1.272-2 \\
1.323-2 \\
1.290-2 \\
1.294-2 \\
1.280-2\end{array}$ & $\begin{array}{l}3.340-2 \\
3.342-2 \\
3.174-2 \\
3.212-2 \\
3.110-2 \\
3.131-2 \\
2.990-2\end{array}$ & $\begin{array}{l}1.210-1 \\
1.310-1 \\
1.160-1 \\
1.390-1 \\
1.340-1 \\
1.350-1 \\
1.240-1\end{array}$ & $\begin{array}{l}3.210-1 \\
3.030-1 \\
3.110-1 \\
3.590-1 \\
3.320-1 \\
3.330-1 \\
3.520-1\end{array}$ & $\begin{array}{l}1.210 \\
1.270 \\
1.400 \\
1.410 \\
1.260 \\
1.360 \\
1.610\end{array}$ & $\begin{array}{l}3.290 \\
3.140 \\
3.870 \\
4.030 \\
3.210 \\
4.030 \\
3.470\end{array}$ \\
\hline
\end{tabular}


TABLE ค-IV

TH-232 OELAYED FISSION SPECTRA

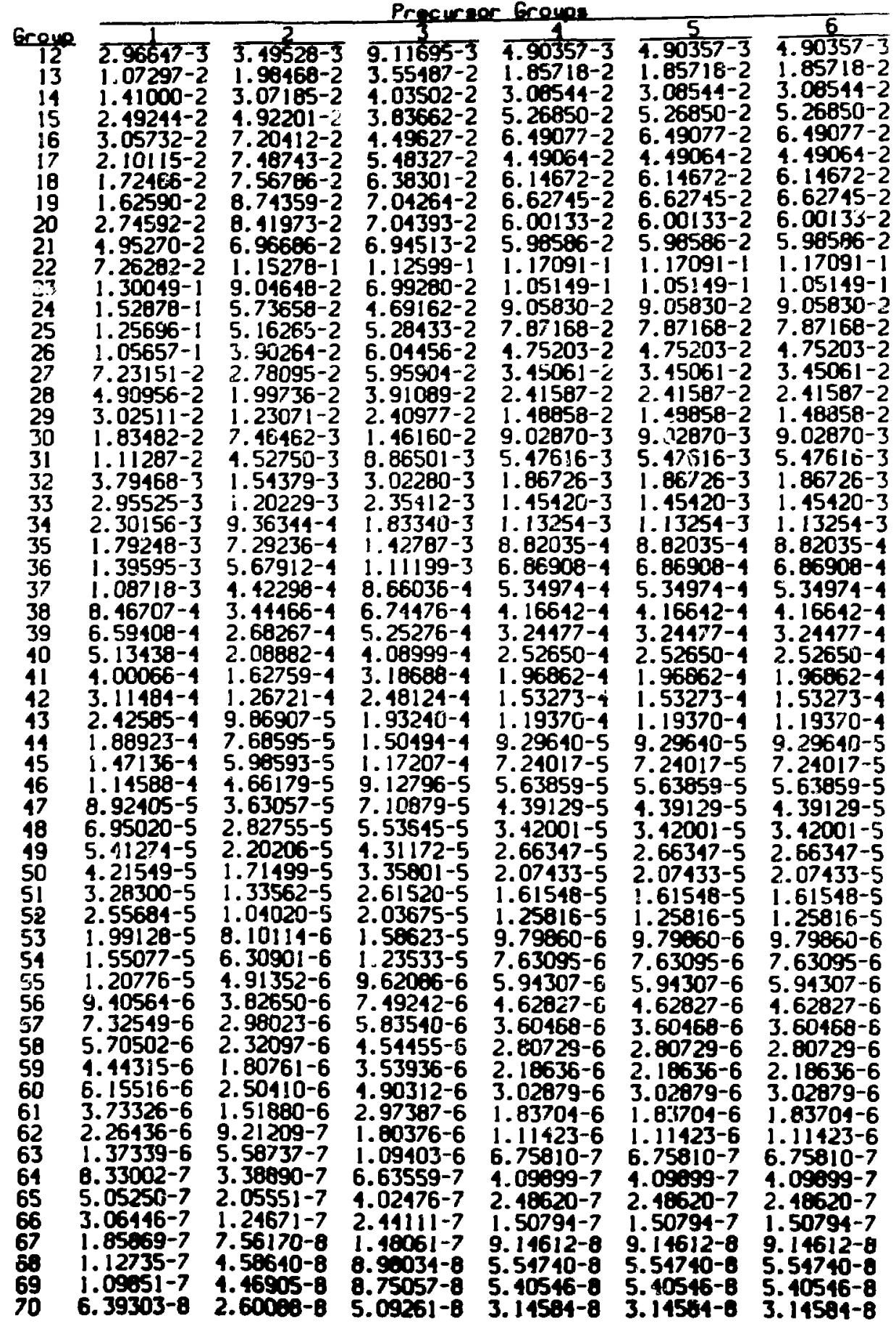


TRQLE $A-V$

\section{U-235 OELAYED FISSION SPECTRA}

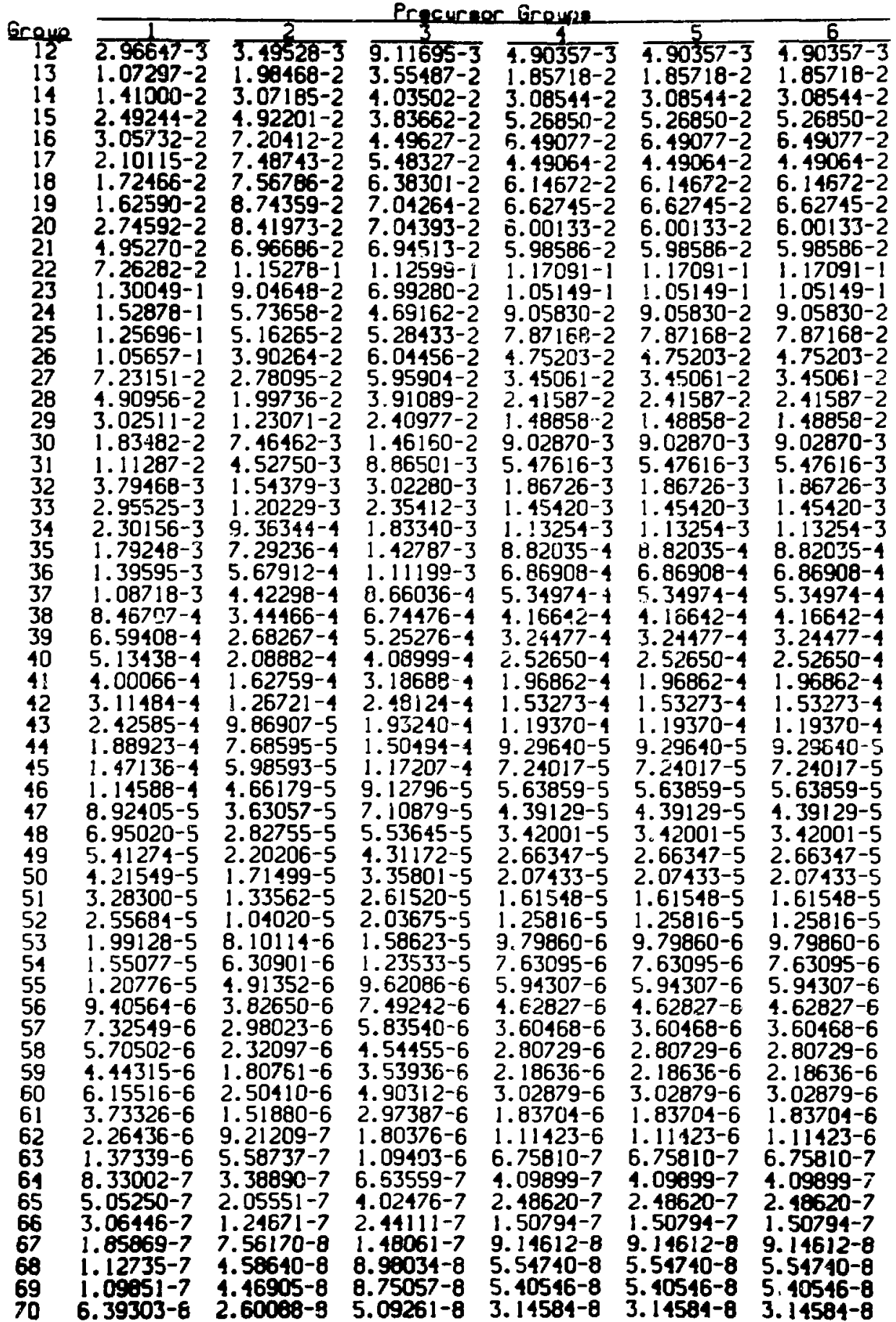


Ifou. A-VI

U-238 DELAYEO FISSION SPECTRA

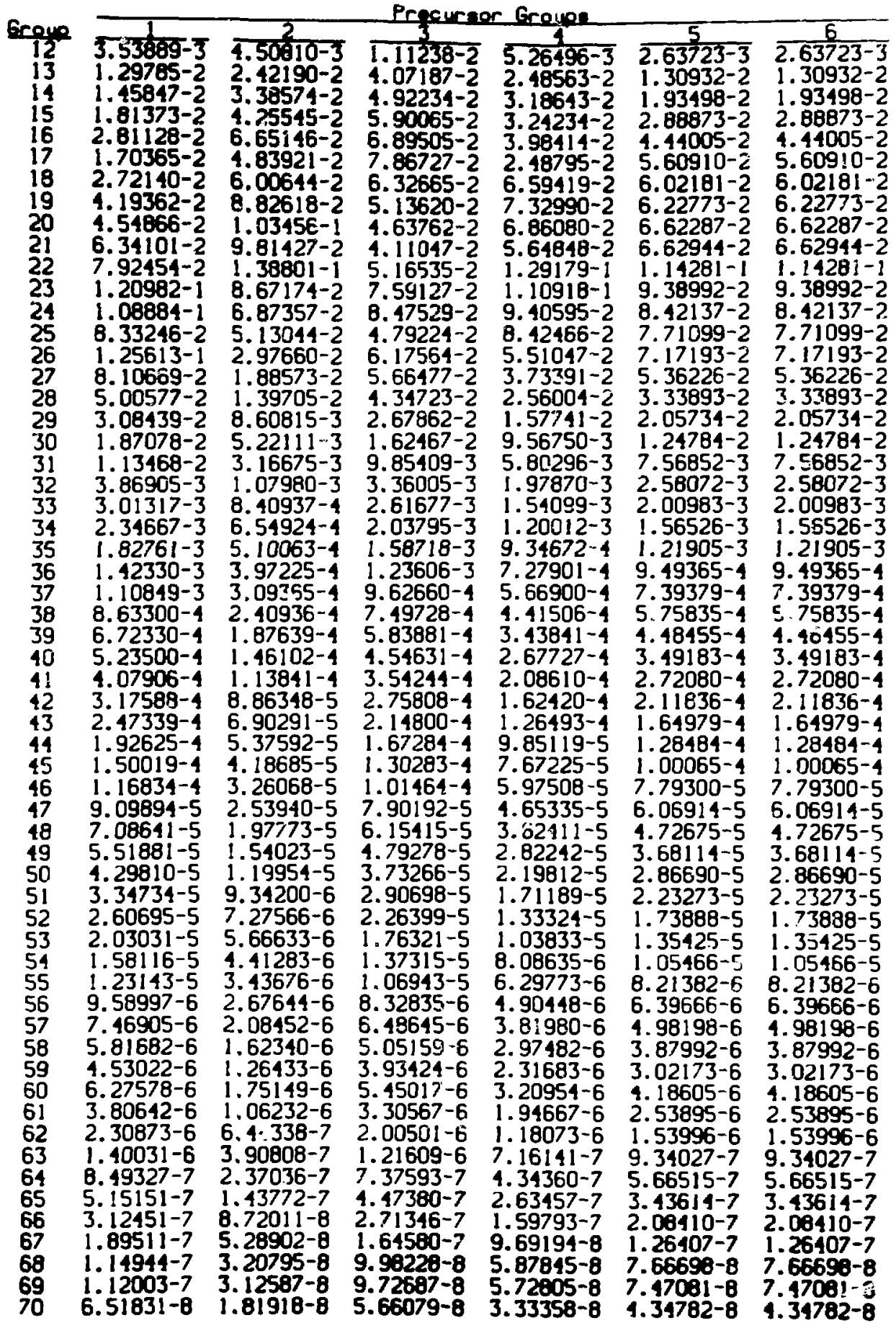


TABLE A-VII

PU-239 DELAYED FISSION SPECTRA

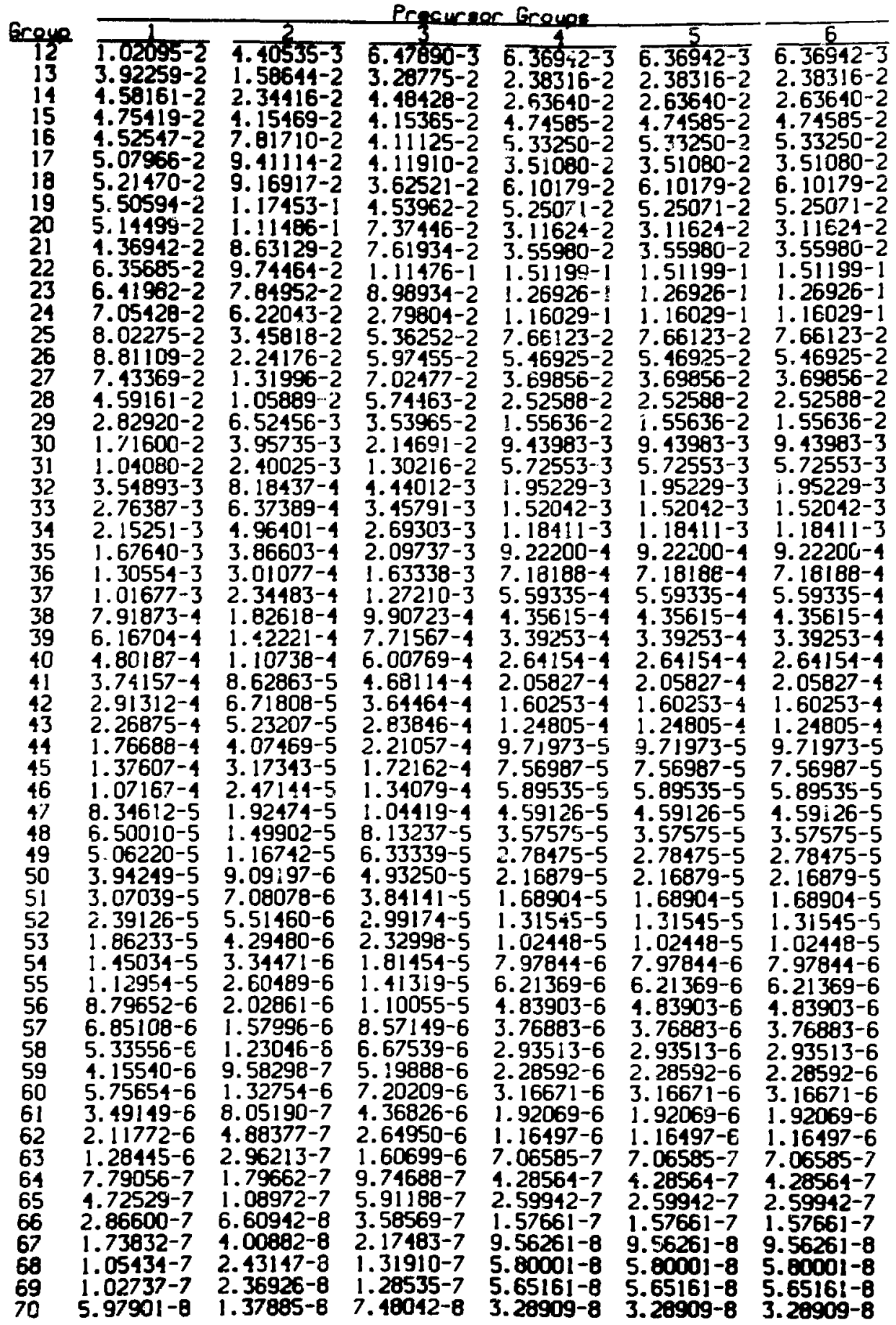




\begin{tabular}{|c|c|c|c|c|c|}
\hline & & P VELO & CITIES $1 \mathrm{~cm}$ & & \\
\hline roup & Yolocity & Grour & Yolocity & Group & Velocil \\
\hline $\begin{array}{l}1 \\
2 \\
3 \\
4 \\
5 \\
6 \\
7 \\
8 \\
9 \\
10 \\
11 \\
12 \\
13 \\
14 \\
15 \\
10 \\
17 \\
18 \\
19 \\
20 \\
21 \\
22 \\
23\end{array}$ & $\begin{array}{l}5.81061+9 \\
5.19350+9 \\
4.59828+9 \\
4.11836+9 \\
3.63445+9 \\
3.20735+9 \\
2.81917+9 \\
2.48793+9 \\
2.20072+9 \\
1.94216+9 \\
1.71395+9 \\
1.56160+9 \\
1.46700+9 \\
1.37811+9 \\
1.29451+9 \\
1.21618+9 \\
1.14250+9 \\
1.07373+9 \\
1.00868+9 \\
9.47568+8 \\
8.90156+8 \\
8.10996+8 \\
7.15701+8\end{array}$ & $\begin{array}{l}24 \\
25 \\
26 \\
27 \\
28 \\
29 \\
30 \\
31 \\
32 \\
33 \\
34 \\
35 \\
36 \\
37 \\
38 \\
39 \\
40 \\
41 \\
42 \\
43 \\
44 \\
45\end{array}$ & $\begin{array}{l}6.31603+8 \\
5.57769+8 \\
4.92576+8 \\
4.34694+8 \\
3.83618+8 \\
3.38542+8 \\
2.98870+8 \\
2.63853+8 \\
2.39711+8 \\
2.25187+8 \\
2.11544+8 \\
1.98727+8 \\
1.86687+8 \\
1.75376+8 \\
1.64752+8 \\
1.51769+8 \\
1.45393+8 \\
1.36584+8 \\
1.28308+8 \\
1.20545+8 \\
1.13241+8 \\
1.06380+8 \\
9.99353+7\end{array}$ & $\begin{array}{l}47 \\
48 \\
49 \\
50 \\
51 \\
52 \\
53 \\
54 \\
55 \\
56 \\
57 \\
58 \\
59 \\
60 \\
6 ! \\
62 \\
63 \\
64 \\
65 \\
66 \\
67 \\
68 \\
69 \\
70\end{array}$ & $\begin{array}{l}9.38804+7 \\
8.81926+7 \\
8.28492+7 \\
7.78296+7 \\
7.31142+7 \\
6.86845+7 \\
6.45229+7 \\
6.06137+7 \\
5.69723+7 \\
5.35209+7 \\
5.02783+7 \\
4.72319+7 \\
4.43706+7 \\
405651+7 \\
3.7986+7 \\
3.5922+7 \\
2.78800+7 \\
2.46310+7 \\
2.17645+7 \\
1.92071+7 \\
1.69502+7 \\
1.49585+7 \\
1.27178+7 \\
9.51308+6\end{array}$ \\
\hline
\end{tabular}




\section{APPENDIX B}

\section{BENCHMARK RESULTS}

This appendix contains the one-dimensional diffusion theory and transport theory results for each benchmark. Tables B-I through B-XVII compare calculated results to the available experimental results.

Tables B-XVIII through B-LXXXI show the 70-group and collapsed 25-group transport theory central fluxes, central adjoints, edge fluxes, edge adjoints, and region fission fractions. The fluxes are normalized so that the central flux sum is 1.0. The adjoints are normalized so that the sum of the product of the central adjoint and the central effective fission source is 1.0 . The reactor fission fraction vectors were obtained by computing the total number of vof neutrons born into each group and then normalizing so that the sum over all groups would be 1.0 . The 25 -group adjoints were obtained by a simple addition of the original fine group adjoints in each coarse group.

Table B-LXXXII through B-XCVIII contain several centrally averaged onegroup cross sections. The fission and "capture" cross sections are simply flux averaged. [Actually, "capture" = capture $-(n, 2 n)-2 \star(n, 3 n)$. However, if the central reaction rate ratios called for an isotope's capture or $(n, \gamma)$ reaction rate, then efforts were taken to make "capture" = capture.] Nu is $\emptyset \sigma_{f}$ averaged, and the transport cross section is $\left(\emptyset_{1}-\phi_{2}\right) / \sum_{t r}$ averaged (where $\phi_{1}$ is the flux at mesh point $i$, and $\Sigma_{t r}$ is the macroscopic transport cross section).

Several symbols used in the tables are defined below.

$\%$ Uncer $=$ per cent error on measured values

A $\quad=$ Rossi Alpha

s $\quad=$ seconds

Beta = effective delayed neutron fraction

E $=$ exponent to the base 10 follows

$\mathrm{L}=$ neutron generation time

$\mathrm{R} / \mathrm{IH}=$ reactivity/in-hour conversion factor

$\$ \quad=$ dollar, another unit of reactivity

$\mathrm{kg}=\mathrm{kil} \log \mathrm{ram}$

$\mathrm{C} / \mathrm{E}=\mathrm{calculated} / \mathrm{experimental}$ ratio 


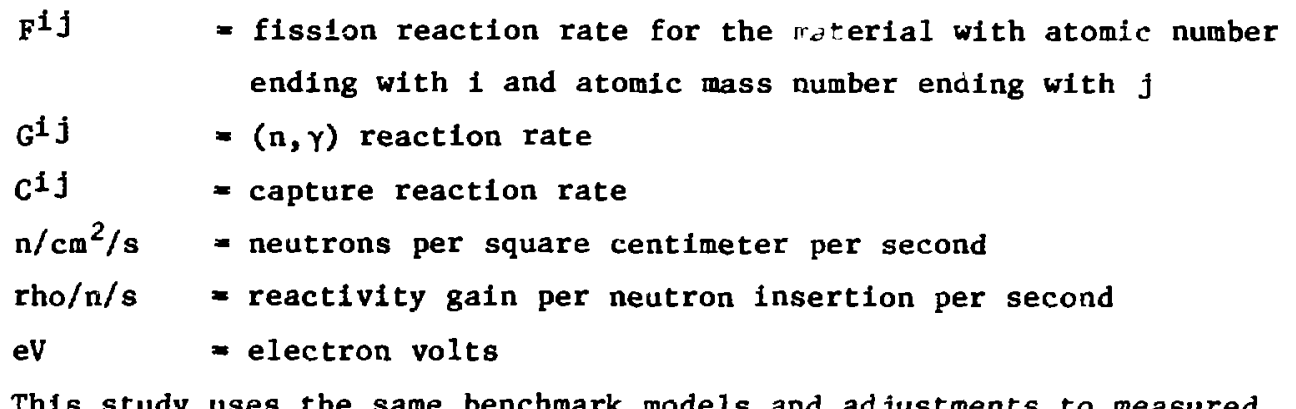

This study uses the same benchmark models and adjustments to measured results as spelled out in Appendix $B$ of $\operatorname{Ref} .5$. 
IFELE B-I

JE2EELL RESULTS

\begin{tabular}{|c|c|c|c|c|c|c|}
\hline \multirow[b]{2}{*}{ Poranler } & \multirow[b]{2}{*}{$\begin{array}{c}\text { Mooeured } \\
\text { Yalue }\end{array}$} & \multirow[b]{2}{*}{ Uncer. } & \multicolumn{2}{|c|}{ Diffueion Theory } & \multicolumn{2}{|c|}{ Ironnoort Theory } \\
\hline & & & $\begin{array}{l}\text { Colculoted } \\
\text { Volwe }\end{array}$ & $C / E$ & $\begin{array}{r}\text { Colculoled } \\
\text { Yalue }\end{array}$ & $c / \varepsilon$ \\
\hline \multicolumn{7}{|c|}{ Minen thomene Pocontere } \\
\hline $\begin{array}{l}\text { Lgenvalue } \\
(/ 5) \\
\text { og }(E-3) \\
\text { (E-9 } 5) \\
\text { / IH (E-6) }\end{array}$ & $\begin{array}{r}1.000 \varepsilon+00 \\
-6.100 \varepsilon+05\end{array}$ & $\begin{array}{l}0.20 \\
1.56\end{array}$ & $\begin{array}{r}9.600 \tau-01 \\
-5.224 E+05 \\
1.87 z E+00 \\
3.583 E+00 \\
7.489 E+00\end{array}$ & $\begin{array}{l}0.9600 \\
0.8163\end{array}$ & $\begin{array}{r}1.011 E+\infty 0 \\
-5.204 E+\infty 5 \\
1.651 E+\infty 0 \\
3.582 E+\infty 0 \\
7.161 E+\infty 0\end{array}$ & $\begin{array}{l}1.0111 \\
0.8131\end{array}$ \\
\hline
\end{tabular}

Caniral Ragetion Rote Rotion

\begin{tabular}{|c|c|c|c|c|c|c|}
\hline $\begin{array}{l}\text { F28/F25 } \\
F 23 / F 25 \\
F 37 / F 25 \\
F 49 / F 25 \\
\text { GV/F25 } \\
\text { GWw55/F25 } \\
\text { GCU/F25 } \\
\text { GNG93/F25 } \\
\text { GAU197/F25 }\end{array}$ & $\begin{array}{l}2.137 \varepsilon-01 \\
1.579 \varepsilon+00 \\
9.620 \tau-01 \\
1.448 \varepsilon+00 \\
2.300 \tau-03 \\
2.400 \tau-03 \\
1.000 \tau-02 \\
2.300 \tau-02 \\
8.300 \tau-02\end{array}$ & $\begin{array}{r}1.08 \\
1.71 \\
1.66 \\
2.00 \\
13.04 \\
12.50 \\
6.00 \\
8.70 \\
2.41\end{array}$ & $\begin{array}{l}1.925 E-01 \\
1.557 E+00 \\
9.402 E-01 \\
1.404 E+00 \\
1.816 E-03 \\
2.895 E-03 \\
0.498 E-03 \\
2.767 E-02 \\
1.059 E-02\end{array}$ & $\begin{array}{l}0.9010 \\
0.9669 \\
0.9774 \\
0.9698 \\
0.7902 \\
1.2064 \\
0.8498 \\
1.203 ! \\
0.9709\end{array}$ & $\begin{array}{l}1.959 E-01 \\
1.557 \mathrm{E}+00 \\
9.515 \mathrm{E}-01 \\
1.408 \mathrm{E}+00 \\
1.782 E-03 \\
2.638 \mathrm{E}-03 \\
8.379 E-03 \\
2.712 \mathrm{E}-02 \\
7.910 E-02\end{array}$ & $\begin{array}{l}0.9169 \\
0.9866 \\
0.9891 \\
0.9724 \\
0.7750 \\
1.1826 \\
0.8379 \\
1.1791 \\
0.9530\end{array}$ \\
\hline
\end{tabular}

Centrol Reactivily Worthe (f/kgl

\begin{tabular}{|c|c|c|c|c|c|c|}
\hline $\begin{array}{l}H-1 \\
B E-9 \\
B-10 \\
C \\
N-14 \\
0-16 \\
A L-27 \\
T 1 \\
V \\
F E \\
C D-59 \\
N I \\
Z R \\
M O \\
A G \\
T A-181 \\
W \\
A U-197 \\
T H-232 \\
U-233 \\
U-235 \\
U-238 \\
N P-237 \\
P U-239 \\
P U-240 \\
A M-241\end{array}$ & $\begin{array}{r}2.089 E+02 \\
1.752 E+01 \\
-2.576 E+02 \\
-6.135 E+00 \\
-1.651 E+01 \\
-6.252 E+00 \\
-5.462 E+00 \\
-5.604 E+00 \\
-3.100 E+00 \\
-3.956 E+00 \\
-4.287 E+00 \\
-8.428 E+00 \\
-4.039 E+00 \\
-4.718 E+00 \\
-8.929 E+00 \\
-5.730 E+00 \\
-4.094 E+00 \\
-4.569 E+00 \\
-2.881 E+00 \\
6.069 E+01 \\
3.538 E+01 \\
4.864 E+00 \\
3.619 E+01 \\
6.944 E+01 \\
4.495 E+01 \\
4.520 E+01\end{array}$ & $\begin{array}{r}50.00 \\
6.67 \\
2.04 \\
14.29 \\
4.55 \\
10.53 \\
7.14 \\
3.92 \\
6.67 \\
4.76 \\
4.17 \\
2.13 \\
2.86 \\
2.33 \\
1.64 \\
1.52 \\
2.10 \\
1.75 \\
1.57 \\
1.00 \\
1.01 \\
1.82 \\
6.13 \\
0.98 \\
4.88 \\
8.21\end{array}$ & $\begin{array}{r}1.111 E+02 \\
1.936 E+01 \\
-1.686 E+02 \\
-1.270 E+00 \\
-2.238 E+01 \\
-2.118 E+00 \\
-1.833 E+00 \\
-2.040 E+00 \\
-1.315 E+00 \\
-1.866 E+00 \\
-1.909 E+00 \\
-4.557 E+00 \\
-1.350 E+00 \\
-2.343 E+00 \\
-4.864 E+00 \\
-3.251 E+00 \\
-1.906 E+00 \\
-2.579 E+00 \\
-1.057 E+00 \\
4.287 E+01 \\
2.539 E+01 \\
3.924 E+00 \\
2.793 E+01 \\
4.862 E+01 \\
3.119 E+01 \\
3.453 E+01\end{array}$ & $\begin{array}{l}1.9680 \\
1.1051 \\
0.6544 \\
0.2070 \\
1.3530 \\
0.3368 \\
0.3356 \\
0.3640 \\
0.1242 \\
0.4715 \\
0.1451 \\
0.5407 \\
0.3343 \\
0.1966 \\
0.5448 \\
0.5674 \\
0.4661 \\
0.5645 \\
0.3670 \\
0.7064 \\
0.7176 \\
0.8068 \\
0.7718 \\
0.7001 \\
0.6938 \\
0.7639\end{array}$ & $\begin{array}{r}8.789 E+00 \\
1.775 E+01 \\
-2.573 E+02 \\
-7.128 E+00 \\
-3.905 E+01 \\
-6.961 E+00 \\
-5.073 E+00 \\
-4.751 E+00 \\
-3.751 E+00 \\
-4.447 E+00 \\
-4.440 E+00 \\
-8.597 E+00 \\
-3.165 E+00 \\
-5.124 E+00 \\
-8.868 E+00 \\
-6.349 E+00 \\
-4.336 E+00 \\
-5.266 E+00 \\
-3.123 E+00 \\
6.178 E+01 \\
3.613 E+01 \\
4.590 E+00 \\
3.964 E+01 \\
7.052 E+01 \\
4.500 E+01 \\
4.968 E+01\end{array}$ & $\begin{array}{l}0.0421 \\
1.0133 \\
0.9988 \\
1.1619 \\
2.3609 \\
1.1134 \\
0.9288 \\
0.8478 \\
1.2101 \\
1.1235 \\
1.0358 \\
1.0200 \\
0.7835 \\
1.0860 \\
0.9931 \\
1.1080 \\
1.0590 \\
1.1530 \\
1.0840 \\
1.0160 \\
1.0212 \\
0.9437 \\
1.0954 \\
1.0156 \\
1.0010 \\
1.0992\end{array}$ \\
\hline
\end{tabular}

Jerebel Leotoge Flux Comporisen

\begin{tabular}{|c|c|c|c|c|c|c|c|c|}
\hline $\begin{array}{r}\text { Fine } \\
\text { Grougs }\end{array}$ & $\begin{array}{r}\text { Enorgy } \\
\text { Boundory } \\
\text { lev } \\
\end{array}$ & $\begin{array}{l}\text { Lelh. } \\
\text { Nidih }\end{array}$ & $\begin{array}{c}\text { Moosured } \\
\text { Flux } \\
\left(\mathrm{n} / \mathrm{cm}^{2} / \mathrm{s}\right) \\
\end{array}$ & $\begin{array}{r}\% \\
\text { Uncer. }\end{array}$ & $\begin{array}{c}\text { Diffurion } \\
\text { Colculoted } \\
\text { Flux } \\
\text { in/cans }\end{array}$ & heory & $\begin{array}{c}\text { Tronsport } \\
\text { Colculoted } \\
\text { flux } \\
\text { (n/cmes }\end{array}$ & heory \\
\hline $\begin{array}{r}4-5 \\
6-7 \\
8-9 \\
10-11 \\
12-15 \\
16-19 \\
20-22\end{array}$ & 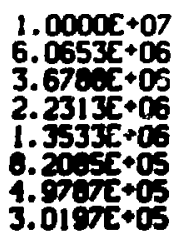 & $\begin{array}{l}0.5 \\
0.5 \\
0.5 \\
0.5 \\
0.5 \\
0.5 \\
0.5\end{array}$ & $\begin{array}{r}3.1 \\
11.7 \\
17.7 \\
20.0 \\
16.5 \\
13.6 \\
9.7\end{array}$ & $\begin{array}{r}16.1 \\
6.0 \\
3.9 \\
4.0 \\
4.2 \\
5.1 \\
7.2\end{array}$ & $\begin{array}{r}2.4795 \\
9.9945 \\
17.7660 \\
20.0000 \\
18.1752 \\
13.0272 \\
8.9477\end{array}$ & $\begin{array}{l}0.7999 \\
0.8542 \\
1.0037 \\
1.0000 \\
1.1015 \\
1.0167 \\
0.9224\end{array}$ & $\begin{array}{r}2.1174 \\
9.8366 \\
17.6194 \\
20.0000 \\
18.3097 \\
14.2556 \\
9.4061\end{array}$ & $\begin{array}{l}0.7798 \\
0.8407 \\
0.9954 \\
1.0000 \\
1.1145 \\
1.0482 \\
0.9697\end{array}$ \\
\hline
\end{tabular}


TABLE B-I!

VERA-IIA RESULIS

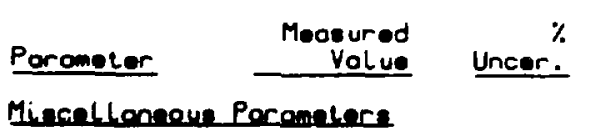

tomeaus Poramelers

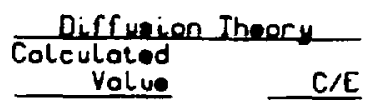

0.30

$$
\begin{array}{r}
9.411 E-01 \\
-4.591 E+00 \\
3.317 E+00 \\
7.225 E+00 \\
1.059 E+00
\end{array}
$$

Centrab Reaction Bale Ratwer

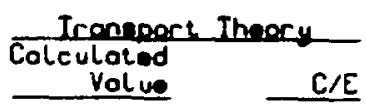

$$
\begin{array}{rr}
0.9411 \quad & 9.884 E-01 \\
& -4.557 E+00 \\
3.047 E+00 \\
6.685 E+00 \\
1.005 E+00
\end{array}
$$

0.9884

\begin{abstract}
8. 748E-C2
1. $169 E+00$

5. $1992-01$

5. $072 E-01$

$1.517 E+00$
\end{abstract}
1.1361
1.0922
1.0945
1.1795
1.0182

$1.490 E+00$

2.01
1.1916

1.1035

1.1373

1.228
TABLE B-111

ZPR-3-48 RESULTS

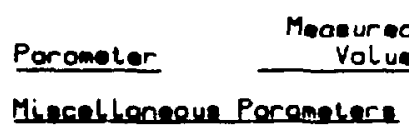

Eisanual $1.0005+0$

$A \mid E 4 / S)$

Bota $(E-3)$

$L(E-7)$

$R / I H(E-5)$

Gentral Reaction Rote Rotion

$\begin{array}{lllllll}C 28 / F 25 & 1.385 E-01 & 5.34 & 1.348 E-01 & 0.9733 & 1.348 E-01 & 0.9733 \\ \text { F28/F25 } & 3.265 E-02 & 4.98 & 3.501 E-02 & 1.0723 & 3.498 E-02 & 1.0714\end{array}$

Cencral Reaclivity Horthe linhoura/tal

$\begin{array}{lrrrrrr}P U-239 & 4.503 E+02 & 0.92 & 5.185 E+02 & 1.1513 & 5.316 E+02 & 1.1806 \\ U-235 & 3.361 E+02 & 1.50 & 3.786 E+02 & 1.1254 & 3.880 E+02 & 1.1533 \\ U-238 & -2.106 E+01 & 2.97 & -2.353 E+01 & 1.1173 & -2.152 E+01 & 1.1643 \\ N A-23 & -6.368 E+00 & 1.73 & -1.431 E+01 & 2.2472 & -1.580 E+01 & 2.4811 \\ B-10 & -8.051 E+03 & 0.67 & -8.336 E+03 & 1.0350 & -8.539 E+03 & 1.0602 \\ F E & -1.230 E+01 & 3.29 & -1.606 E+01 & 1.3057 & -1.695 E+01 & 1.3781 \\ C R & -1.230 E+01 & 12.12 & -2.070 E+01 & 1.6832 & -2.167 E+01 & 1.7617 \\ N I & -1.821 E+01 & 0.92 & -2.463 E+01 & 1.3527 & -2.569 E+01 & 1.4108 \\ W N-55 & -2.286 E+01 & 1.69 & -2.978 E+01 & 1.3025 & -3.095 E+01 & 1.3539 \\ A K-27 & -1.571 E+01 & 5.09 & -2.214 E+01 & 1.4095 & -2.347 E+01 & 1.4938 \\ T A-181 & -1.640 E+02 & 3.04 & -1.658 E+02 & 1.0109 & -1.701 E+02 & 1.0373 \\ M O & -4.335 E+01 & 0.94 & -5.650 E+01 & 1.3033 & -5.837 E+01 & 1.3464 \\ C & -4.496 E+00 & 27.27 & -2.428 E+01 & 5.4006 & -2.690 E+01 & 5.9927\end{array}$


TABLE B-IV

ZEBRA-3 RESULIS

\begin{tabular}{|c|c|}
\hline Parameter & $\begin{array}{l}\text { Meosured } \\
\text { Vol ue }\end{array}$ \\
\hline \multicolumn{2}{|c|}{ Milentlonneus Porameters } \\
\hline $\begin{array}{l}\text { ELgenvol ue } \\
\text { A PES/S) } \\
\text { Beto (E-3) } \\
L(E-8 S] \\
R /] H(E-5)\end{array}$ & $1.000 E+00$ \\
\hline
\end{tabular}
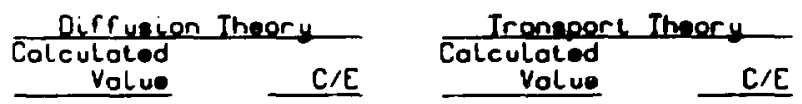

Central Reoction Role Rotwes

$\begin{array}{lllllll}F 28 / F 25 & 4.610 E-02 & 1.74 & 4.777 E-02 & 1.0363 & 4.745 E-02 & 1.0293 \\ \text { F23/F25 } & 1.542 E+00 & 1.23 & 1.558 E+00 & 1.0104 & 1.558 E+00 & 1.0106 \\ \text { F24/F25 } & 3.460 E-01 & 2.60 & 3.888 E-01 & 1.1236 & 3.882 E-01 & 1.1220 \\ F 26 / F 25 & 9.900 E-02 & 5.05 & 1.142 E-01 & 1.1538 & 1.136 E-01 & 1.1478 \\ F 49 / F 25 & 1.190 E+00 & 1.18 & 1.201 E+00 & 1.0091 & 1.201 E+00 & 1.0092 \\ F 40 / F 25 & 3.730 E-01 & 1.34 & 1.068 E-01 & 1.0907 & 1.060 E-01 & 1.0885 \\ F 37 / F 25 & 3.530 E-01 & 1.13 & 3.953 E-01 & 1.1198 & 3.945 E-01 & 1.1175\end{array}$

Centrol Reacleyity Horthe lynheurs/kgl

$\begin{array}{lrrrrrr}U-235 & 7.208 E+02 & 2.03 & 8.121 E+02 & 1.1267 & 8.263 E+02 & 1.1464 \\ P U-239 & 1.144 E+03 & 2.52 & 1.328 E+03 & 1.1607 & 1.353 E+03 & 1.1831 \\ U-238 & -3.595 E+01 & 4.82 & -3.582 E+01 & 0.9964 & -3.877 E+01 & 1.0785 \\ B-10 & -9.018 E+03 & 4.76 & -8.472 E+03 & 0.9395 & -8.728 E+03 & 0.9678 \\ B & -2.127 E+03 & 2.25 & -6.968 E+03 & 3.2758 & -7.181 E+03 & 3.3759 \\ T A-181 & 1.426 E+02 & 3.33 & -1.475 E+02 & -1.0342 & -1.534 E+02 & -1.0755 \\ L I-6 & -1.215 E+04 & 4.71 & -1.066 E+04 & 0.8770 & -1.101 E+04 & 0.9062 \\ A U-197 & -1.135 E+02 & 3.85 & -1.206 E+02 & 1.0626 & -1.256 E+02 & 1.1064 \\ C U & -8.661 E+01 & 4.69 & -9.606 E+01 & 1.1091 & -1.004 E+02 & 1.1590 \\ C & -2.723 E+02 & 5.26 & -2.908 E+02 & 1.0679 & -3.146 E+02 & 1.1554 \\ N A-23 & -1.047 E+02 & 10.70 & -1.375 E+02 & 1.3136 & -1.481 E+02 & 1.4114 \\ \text { AL-27 } & -1.084 E+02 & 11.76 & -1.140 E+02 & 1.0519 & -1.212 E+02 & 1.1180 \\ P B & -1.619 E+01 & 10.26 & -1.885 E+01 & 1.1645 & -1.976 E+01 & 1.2208 \\ H-1 & -2.731 E+04 & 3.13 & -4.045 E+04 & 1.9814 & -4.300 E+04 & 1.5749\end{array}$


IABLE B-V

GODIVA RESULTS

\begin{tabular}{|c|c|c|c|c|c|c|}
\hline \multirow[b]{2}{*}{ Porometer } & \multirow[b]{2}{*}{$\begin{array}{r}\text { Moosured } \\
\text { Volue }\end{array}$} & \multirow[b]{2}{*}{$\begin{array}{r}\% \\
\text { Uncer. }\end{array}$} & \multicolumn{2}{|c|}{ Diffuruen Theory } & \multicolumn{2}{|c|}{ Ironeport Theory } \\
\hline & & & $\begin{array}{r}\text { Colculoted } \\
\text { Volve } \\
\end{array}$ & $C / E$ & $\begin{array}{r}\text { Colculoled } \\
\text { volue }\end{array}$ & $C / E$ \\
\hline \multicolumn{7}{|c|}{ Mincaltonequs Poromere } \\
\hline $\begin{array}{l}\text { Eiganvol ue } \\
\text { A }(15) \\
\theta \text { oto }(E-3) \\
L(E-9 S) \\
R / I H \text { (E) }\end{array}$ & $\begin{array}{r}1.000 E+00 \\
-1.110 E+06\end{array}$ & $\begin{array}{l}0.10 \\
1.80\end{array}$ & $\begin{array}{r}9.666 E-01 \\
-1.132 \mathrm{E}+06 \\
6.613 \mathrm{0}+00 \\
5.842 \mathrm{E}+00 \\
2.303 \mathrm{0}+00\end{array}$ & $\begin{array}{l}0.9666 \\
1.0197\end{array}$ & $\begin{array}{r}. .003 \mathrm{E}+00 \\
-1.129 \mathrm{E}+06 \\
6.555 \mathrm{E}+00 \\
5.808 \mathrm{E}+00 \\
2.282 \mathrm{E}+00\end{array}$ & $\begin{array}{l}1.3028 \\
1.5167\end{array}$ \\
\hline \multicolumn{7}{|c|}{ Central Reaction Role Roter } \\
\hline $\begin{array}{l}\text { F28/F } 25 \\
\text { F23/F25 } \\
F 37 / F 25 \\
F 49 / F 25 \\
\text { GHN55/F25 } \\
\text { GCOS9/F25 } \\
\text { GCU/F25 } \\
\text { GHB93/F25 } \\
\text { GRU197/F25 }\end{array}$ & $\begin{array}{l}1.647 E-01 \\
1.590 E+00 \\
8.370 E-01 \\
1.402 E+00 \\
2.700 E-03 \\
3.800 E-02 \\
1.170 E-02 \\
3.000 E-02 \\
1.000 E-01\end{array}$ & $\begin{array}{r}1.09 \\
1.89 \\
1.55 \\
1.78 \\
7.41 \\
7.89 \\
5.13 \\
10.00 \\
2.00\end{array}$ & $\begin{array}{l}1.677 E-01 \\
1.568 E+00 \\
8.807 E-01 \\
1.39 ! E+00 \\
3.049 E-03 \\
5.923 E-03 \\
8.881 E-03 \\
3.003 E-02 \\
8.672 E-02\end{array}$ & $\begin{array}{l}1.0181 \\
0.9861 \\
1.0522 \\
0.9921 \\
1.1292 \\
0.1559 \\
0.7591 \\
1.0010 \\
0.8672\end{array}$ & $\begin{array}{l}1.707 E-01 \\
1.567 E+00 \\
8.906 E-01 \\
1.391 E+00 \\
3.006 E-03 \\
5.670 E-03 \\
8.790 E-03 \\
2.958 E-02 \\
8.551 E-02\end{array}$ & $\begin{array}{l}1.0365 \\
0.9857 \\
1.0641 \\
0.9943 \\
1.1132 \\
0.1545 \\
0.7513 \\
0.9860 \\
0.8551\end{array}$ \\
\hline \multicolumn{7}{|c|}{ Central Reoctively Worthe (S/kg) } \\
\hline $\begin{array}{l}H-1 \\
B E-9 \\
B-10 \\
C \\
A L-27 \\
F E \\
C D-59 \\
M I \\
C U \\
R U-197 \\
T H-232 \\
U-235 \\
U-238 \\
P U-239 \\
P U-240\end{array}$ & $\begin{array}{r}3.692 E+02 \\
8.086 E+00 \\
-5.884 E+01 \\
2.065 E+00 \\
1.724 E-01 \\
-2.776 E-02 \\
-1.052 E-01 \\
-7.924 E-01 \\
-2.928 E-01 \\
-3.936 E-01 \\
-6.013 E-02 \\
6.662 E+00 \\
1.055 E+00 \\
1.254 E+01 \\
7.427 E+00\end{array}$ & $\begin{array}{r}12.50 \\
14.89 \\
5.26 \\
12.50 \\
66.67 \\
200.00 \\
50.00 \\
6.67 \\
16.67 \\
4.00 \\
22.22 \\
0.99 \\
1.85 \\
1.03 \\
10.43\end{array}$ & $\begin{array}{r}3.473 E+02 \\
7.539 E+00 \\
-3.746 E+01 \\
1.520 E+00 \\
2.392 E-01 \\
8.531 E-03 \\
4.522 E-03 \\
-4.983 E-01 \\
-4.471 E-02 \\
-1.532 E-01 \\
1.731 E-01 \\
5.175 E+00 \\
9.490 E-01 \\
9.801 E+00 \\
6.113 E+00\end{array}$ & $\begin{array}{r}0.9408 \\
0.9323 \\
0.6366 \\
0.7362 \\
1.3874 \\
-0.3073 \\
-0.0430 \\
0.6289 \\
0.1527 \\
0.3893 \\
-2.8793 \\
0.7768 \\
0.8996 \\
0.7816 \\
0.8231\end{array}$ & $\begin{array}{r}3.638 E+02 \\
7.960 E+00 \\
-4.910 E+01 \\
1.089 E+00 \\
-5.683 E-02 \\
-2.378 E-01 \\
-2.351 E-01 \\
-9.074 E-01 \\
-3.553 E-01 \\
-4.077 E-01 \\
3.086 E-03 \\
6.527 E+00 \\
1.058 E+00 \\
1.253 E+01 \\
7.777 E+00\end{array}$ & $\begin{array}{r}0.9853 \\
0.9844 \\
0.8345 \\
0.5273 \\
-0.3296 \\
8.5671 \\
2.2345 \\
1.1451 \\
1.2133 \\
1.0358 \\
-0.0513 \\
0.9798 \\
1.0028 \\
0.9990 \\
1.0472\end{array}$ \\
\hline
\end{tabular}

Godivo Leakege $\mathrm{Flux}$ Comperuson

\begin{tabular}{|c|c|c|c|c|c|c|c|c|}
\hline $\begin{array}{r}\text { Fine } \\
\text { Groups } \\
\end{array}$ & $\begin{array}{r}\text { Energy } \\
\text { Boundory } \\
\text { levl } \\
\end{array}$ & $\begin{array}{l}\text { Loth. } \\
\text { WLdih }\end{array}$ & $\begin{array}{c}\text { Mensured } \\
r(\text { Lux } \\
\left|\mathrm{n} / \mathrm{cm}^{2} / \mathrm{s}\right| \\
\end{array}$ & $\begin{array}{r}\% \\
\text { Uncer }\end{array}$ & $\begin{array}{c}\text { Defrusion } \\
\text { Colculoted } \\
\text { rlux } \\
{\left[\mathrm{n} / \mathrm{cm}^{2 / s} \text { ) }\right.}\end{array}$ & eer $y$ & $\begin{array}{c}\text { Tronspert } \\
\text { Colculoted } \\
\text { Plux } \\
\left(\mathrm{n} / \mathrm{cm}^{2} / \mathrm{s}\right)\end{array}$ & heory \\
\hline $\begin{array}{r}4-5 \\
6-7 \\
8-9 \\
10-11 \\
12-15 \\
16-19 \\
20-22 \\
23-24\end{array}$ & $\begin{array}{l}1.0000 E+07 \\
6.0653 E+06 \\
3.6788 E+06 \\
2.2313 E+06 \\
1.3533 E+06 \\
8.2085 E+05 \\
1.9787 E+05 \\
3.0197 E+05 \\
1.8316 E+05\end{array}$ & $\begin{array}{l}0.5 \\
0.5 \\
0.5 \\
0.5 \\
0.5 \\
0.5 \\
0.5 \\
0.5\end{array}$ & $\begin{array}{r}2.0 \\
7.8 \\
13.6 \\
16.8 \\
18.0 \\
17.5 \\
12.0 \\
7.2\end{array}$ & $\begin{array}{l}15.0 \\
5.1 \\
3.7 \\
3.6 \\
3.3 \\
4.0 \\
5.8 \\
9.7\end{array}$ & $\begin{array}{r}1.9232 \\
8.0060 \\
15.5820 \\
18.9112 \\
18.0000 \\
14.7528 \\
10.2845 \\
5.7404\end{array}$ & $\begin{array}{l}0.9616 \\
1.0264 \\
1.1457 \\
1.1257 \\
1.0000 \\
0.8430 \\
0.8570 \\
0.7973\end{array}$ & $\begin{array}{r}1.8255 \\
7.7217 \\
15.2334 \\
18.6916 \\
18.0000 \\
15.6717 \\
10.7501 \\
6.1062\end{array}$ & $\begin{array}{l}0.9127 \\
0.9900 \\
1.1201 \\
1.1126 \\
1.0000 \\
0.8612 \\
0.8958 \\
0.8481\end{array}$ \\
\hline
\end{tabular}


TABLE B-VI

VERA-1B RESULTS

\begin{tabular}{|c|c|c|c|c|c|c|}
\hline \multirow[b]{2}{*}{ Porometer } & \multirow[b]{2}{*}{$\begin{array}{r}\text { Moosur od } \\
\text { Volue }\end{array}$} & \multirow[b]{2}{*}{$\begin{array}{r}\% \\
\text { Uncer. }\end{array}$} & \multicolumn{2}{|c|}{ Diffureon Iheory } & \\
\hline & & & $\begin{array}{r}\text { Colculoted } \\
\text { Volue } \\
\end{array}$ & $C / E$ & $\begin{array}{r}\text { Colculated } \\
\text { Yolue } \\
\end{array}$ & $C / E$ \\
\hline \multicolumn{7}{|c|}{ Mincelboneves Porometers } \\
\hline $\begin{array}{l}\text { Eiganval ue } \\
\text { A }(1 / S) \\
\text { Boto (E-3) } \\
L / E-7 S \text { ] } \\
R / I H \text { (E-5) }\end{array}$ & $\begin{array}{r}1.000 E+00 \\
-6.900 E+04\end{array}$ & $\begin{array}{l}0.28 \\
2.98\end{array}$ & $\begin{array}{r}9.705 E-01 \\
-7.192 E+04 \\
7.666 E+00 \\
1.066 E+00 \\
2.509 E+00\end{array}$ & $\begin{array}{l}0.9705 \\
1.0423\end{array}$ & $\begin{array}{r}9.923 E-01 \\
-7.311 E+04 \\
7.537 E+00 \\
1.031 E+00 \\
2.489 E+00\end{array}$ & $\begin{array}{l}0.9923 \\
1.0595\end{array}$ \\
\hline \multicolumn{7}{|c|}{ Central Reaction Rote Ratuos } \\
\hline $\begin{array}{l}F 28 / F 25 \\
F 23 / F 25 \\
F 26 / F 25 \\
F 49 / F 25 \\
F 40 / F 25 \\
F 97 / F 25 \\
C 28 / F 25\end{array}$ & $\begin{array}{l}5.650 \varepsilon-02 \\
1.433 E+00 \\
1.340 E-01 \\
1.070 E+00 \\
3.990 E-01 \\
3.800 E-01 \\
1.310 E-01\end{array}$ & $\begin{array}{l}1.50 \\
3.78 \\
7.46 \\
2.43 \\
8.02 \\
3.16 \\
4.58\end{array}$ & $\begin{array}{l}8.042 E-02 \\
1.508 E+00 \\
1.761 E-01 \\
1.151 E+00 \\
1.932 E-01 \\
4.797 E-01 \\
1.249 E-01\end{array}$ & $\begin{array}{l}1.2094 \\
1.0527 \\
1.3145 \\
1.0759 \\
1.2362 \\
1.2623 \\
0.9534\end{array}$ & $\begin{array}{l}\text { 8. } 200 E-02 \\
1.510 E+00 \\
1.795 E-01 \\
1.157 \mathrm{E}+00 \\
5.016 E-01 \\
1.883 \mathrm{E}-01 \\
1.236 E-01\end{array}$ & $\begin{array}{l}1.2331 \\
1.0537 \\
1.3397 \\
1.0813 \\
1.2571 \\
1.2850 \\
0.9438\end{array}$ \\
\hline \multicolumn{7}{|c|}{ 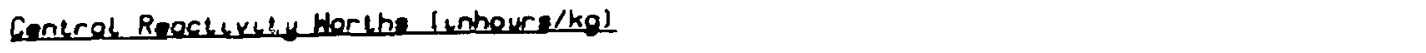 } \\
\hline $\begin{array}{l}U-235 \\
U-238 \\
P U-239 \\
U-233 \\
N P-237 \\
B-10 \\
\text { AU-197 } \\
S . \quad \text { STEEL } \\
A L-27 \\
N A-23 \\
C \\
H-1\end{array}$ & $\begin{array}{r}3.911 E+02 \\
1.311 E+01 \\
6.735 E+02 \\
6.748 E+02 \\
4.911 E+01 \\
-9.846 E+03 \\
-5.191 E+01 \\
1.579 E+01 \\
5.396 E+01 \\
2.352 E+02 \\
2.063 E+C 2 \\
3.715 E+04\end{array}$ & $\begin{array}{r}1.36 \\
4.00 \\
1.29 \\
1.32 \\
10.71 \\
21.10 \\
3.85 \\
1.29 \\
1.43 \\
7.59 \\
2.02 \\
2.22\end{array}$ & $\begin{array}{r}3.714 E+02 \\
2.086 E+01 \\
6.566 E+02 \\
6.716 E+02 \\
9.025 E+01 \\
-9.685 E+03 \\
-7.333 E+01 \\
1.627 E+01 \\
5.624 E+01 \\
8.238 E+01 \\
2.147 E+02 \\
3.726 E+04\end{array}$ & $\begin{array}{l}0.9496 \\
1.5914 \\
0.9749 \\
0.9953 \\
1.8367 \\
0.9836 \\
1.3355 \\
1.0301 \\
1.0423 \\
0.3503 \\
1.0409 \\
1.0029\end{array}$ & $\begin{array}{r}3.940 E+02 \\
2.037 E+01 \\
7.004 E+02 \\
7.132 E+02 \\
1.007 E+02 \\
-1.014 E+04 \\
-7.938 E+01 \\
1.339 E+01 \\
5.339 E+01 \\
7.931 E+01 \\
2.120 E+02 \\
3.807 E+04\end{array}$ & $\begin{array}{l}1.0074 \\
1.5535 \\
1.0400 \\
1.0569 \\
2.0492 \\
1.0298 \\
1.1457 \\
0.8482 \\
0.9895 \\
0.3372 \\
1.0276 \\
1.0248\end{array}$ \\
\hline
\end{tabular}


TABLE $B-V I I$

ZPR-3-6F RESULTS
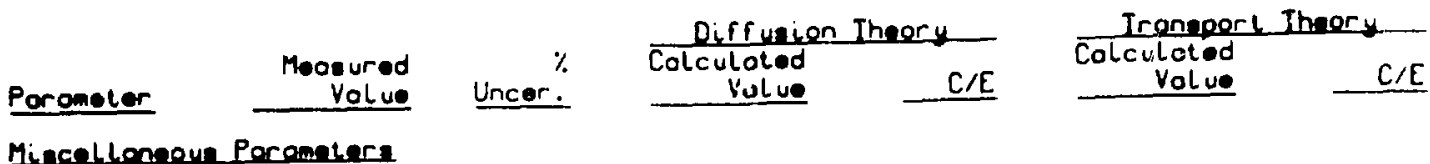

Minaelloneme Poranetera

\begin{tabular}{|c|c|c|c|c|c|c|}
\hline $\begin{array}{l}\text { Eigenvalue } \\
\text { A (1S) } \\
\text { Beto (E-3) } \\
L \text { (E-QS) } \\
R / I H(E-5)\end{array}$ & $\begin{array}{r}1.000 E+00 \\
-9.850 E+04\end{array}$ & $\begin{array}{r}0.15 \\
10.00\end{array}$ & $\begin{array}{r}9.915 E-01 \\
-1.012 E+05 \\
7.411 E+00 \\
7.351 E+00 \\
2.315 E+00\end{array}$ & $\begin{array}{l}0.9915 \\
1.0277\end{array}$ & $\begin{array}{r}1.010 E+00 \\
-1.033 E+05 \\
7.365 E+00 \\
7.131 E+00 \\
2.303 E+00\end{array}$ & $\begin{array}{l}1.0101 \\
1.0485\end{array}$ \\
\hline \multicolumn{7}{|c|}{ Centrol Reaclion Rate Rotwes } \\
\hline $\begin{array}{l}F 28 / F 25 \\
F 24 / F 25 \\
F 23 / F 25 \\
F 49 / F 25 \\
F 40 / F 25 \\
628 / F 25\end{array}$ & $\begin{array}{l}7.800 E-02 \\
4.510 E-01 \\
1.530 E+00 \\
1.220 E+00 \\
5.300 E-01 \\
1.040 E-01\end{array}$ & $\begin{array}{l}2.56 \\
4.43 \\
1.96 \\
2.46 \\
3.77 \\
2.88\end{array}$ & $\begin{array}{l}8.012 E-02 \\
5.240 E-01 \\
1.564 E+00 \\
1.264 E+00 \\
5.590 E-01 \\
9.670 E-02\end{array}$ & $\begin{array}{l}1.0271 \\
1.1619 \\
1.0222 \\
1.0362 \\
1.0547 \\
0.9298\end{array}$ & $\begin{array}{l}\text { 8. } 067 E-02 \\
5.287 E-01 \\
1.565 E+00 \\
1.267 E+00 \\
5.639 E-01 \\
9.628 E-02\end{array}$ & $\begin{array}{l}1.0342 \\
1.1724 \\
1.0229 \\
1.0384 \\
1.0639 \\
0.9258\end{array}$ \\
\hline
\end{tabular}

Centrel Regcturely Worths lenhours/kgl

\begin{tabular}{|c|c|c|c|c|c|c|}
\hline $\begin{array}{l}U-235 \\
U-238 \\
P U \\
U-233 \\
T H-232 \\
B-10 \\
H F \\
T A-181 \\
W \\
M D \\
Z R \\
F E \\
C R \\
N I \\
M N-55 \\
Y \\
N B-93 \\
\text { PL-27 } \\
N A-23 \\
C \\
B E-9 \\
H-1\end{array}$ & $\begin{array}{r}3.202 E+02 \\
2.710 E+00 \\
4.514 E+02 \\
4.502 E+02 \\
-2.224 E+01 \\
-3.693 E+03 \\
-3.373 E+01 \\
-4.040 E+01 \\
-1.731 E+01 \\
1.614 E+01 \\
-4.714 E-01 \\
-6.930 E+00 \\
-4.584 E+00 \\
-1.245 E+01 \\
-5.088 E+00 \\
1.013 E+01 \\
-2.684 E+01 \\
3.665 E+00 \\
5.985 E+01 \\
1.003 E+02 \\
2.338 E+02 \\
2.389 E+04\end{array}$ & $\begin{array}{r}2.86 \\
33.33 \\
4.78 \\
9.84 \\
16.67 \\
5.81 \\
35.71 \\
17.65 \\
21.62 \\
16.67 \\
200.00 \\
33.33 \\
34.55 \\
29.41 \\
36.92 \\
33.33 \\
20.69 \\
152.17 \\
28.13 \\
42.86 \\
32.65 \\
41.07\end{array}$ & $\begin{array}{r}2.692 E+02 \\
8.999 E+00 \\
4.703 E+02 \\
4.718 E+02 \\
-1.657 E+01 \\
-3.388 E+03 \\
-4.575 E+01 \\
-4.157 E+01 \\
-1.488 E+01 \\
-1.974 E+01 \\
-7.173 E+00 \\
-9.160 E+00 \\
-1.144 E+01 \\
-2.125 E+01 \\
-2.843 E-01 \\
3.979 E+00 \\
-2.719 E+01 \\
2.293 E+00 \\
2.650 E+01 \\
8.295 E+01 \\
2.622 E+02 \\
1.562 E+04\end{array}$ & $\begin{array}{r}0.8408 \\
3.3207 \\
1.0419 \\
1.0481 \\
0.7452 \\
0.9173 \\
1.3562 \\
1.0290 \\
0.8598 \\
-1.2231 \\
15.2169 \\
1.3218 \\
2.4947 \\
1.7066 \\
0.0559 \\
0.3928 \\
1.0132 \\
0.6257 \\
0.4428 \\
0.8270 \\
1.1215 \\
0.6537\end{array}$ & $\begin{array}{r}2.818 E+02 \\
7.931 E+00 \\
4.947 E+02 \\
4.952 E+02 \\
-1.943 E+01 \\
-3.567 E+03 \\
-4.985 E+01 \\
-1.577 E+01 \\
-1.757 E+01 \\
-2.294 E+01 \\
-9.029 E+00 \\
-1.158 E+01 \\
-1.400 E+01 \\
-2.462 E+01 \\
-2.737 E+00 \\
1.419 E+00 \\
-3.129 E+01 \\
-1.004 E+00 \\
2.171 E+01 \\
7.487 E+01 \\
2.509 E+02 \\
1.526 E+04\end{array}$ & $\begin{array}{r}0.8800 \\
2.9267 \\
1.0959 \\
1.1000 \\
0.8736 \\
0.9659 \\
1.4778 \\
1.1330 \\
1.0150 \\
-1.4212 \\
19.1536 \\
1.6704 \\
3.0545 \\
1.9776 \\
0.5378 \\
0.1401 \\
1.1658 \\
-0.2739 \\
0.3627 \\
0.7464 \\
1.0731 \\
0.6388\end{array}$ \\
\hline
\end{tabular}


TABLE B-VIII

ZPR-3-11 RESULTS

\begin{tabular}{|c|c|c|c|c|c|c|}
\hline \multirow[b]{2}{*}{ Poromeler } & \multirow[b]{2}{*}{$\begin{array}{l}\text { Meosurad } \\
\text { Yal ue }\end{array}$} & \multirow[b]{2}{*}{$\begin{aligned} \% \\
\text { Uncor. }\end{aligned}$} & \multicolumn{2}{|l|}{ Diff yeion } & \\
\hline & & & $\begin{array}{r}\text { Colculoted } \\
\text { Yolue } \\
\end{array}$ & $C / E$ & $\begin{array}{r}\text { Colculoted } \\
\text { Vol we } \\
\end{array}$ & $C / E$ \\
\hline \multicolumn{7}{|c|}{ Miscellonewe Poromelers } \\
\hline $\begin{array}{l}\text { Eigonval uo } \\
\text { A Y/S) } \\
\text { Beto (E-3) } \\
L \text { (E-a } 5) \\
R / I H \text { (E-5) }\end{array}$ & $\begin{array}{r}1.000 E+00 \\
-1.040 E+05\end{array}$ & $\begin{array}{l}0.25 \\
2.88\end{array}$ & $\begin{array}{r}1.006 E+00 \\
-1.107 E+05 \\
7.268 E+00 \\
6.567 E+00 \\
2.087 E+00\end{array}$ & $\begin{array}{l}1.0062 \\
1.0643\end{array}$ & $\begin{array}{r}1.012 E+00 \\
-1.112 E+05 \\
7.251 E+00 \\
6.519 E+00 \\
2.083 E+00\end{array}$ & $\begin{array}{l}1.0121 \\
1.0695\end{array}$ \\
\hline \multicolumn{7}{|c|}{ Gentral Reachion Role Rolies } \\
\hline $\begin{array}{l}F 28 / F 25 \\
F 24 / F 25 \\
F 23 / F 25 \\
F 49 / F 25 \\
F 40 / F 25 \\
F 26 / F 25 \\
F 97 / F 25 \\
628 / F 25\end{array}$ & $\begin{array}{l}3.800 E-02 \\
3.100 E-01 \\
1.520 E+00 \\
1.190 E+00 \\
3.400 E-01 \\
1.200 E-01 \\
3.300 E-01 \\
1.120 E-01\end{array}$ & $\begin{array}{r}2.63 \\
9.68 \\
1.32 \\
1.68 \\
5.88 \\
16.67 \\
6.06 \\
4.46\end{array}$ & $\begin{array}{l}4.099 E-02 \\
3.621 \mathrm{E}-01 \\
1.558 \mathrm{E}+00 \\
1.191 \mathrm{E}+00 \\
3.772 \mathrm{E}-01 \\
1.006 \mathrm{E}-01 \\
3.619 \mathrm{E}-01 \\
1.085 \mathrm{E}-01\end{array}$ & $\begin{array}{l}1.0786 \\
1.1679 \\
1.0247 \\
1.0009 \\
1.1095 \\
0.8382 \\
1.1057 \\
0.9689\end{array}$ & $\begin{array}{l}4.083 E-02 \\
3.615 E-01 \\
1.558 E+00 \\
1.191 E+00 \\
3.765 E-01 \\
1.003 E-01 \\
3.642 E-01 \\
1.066 E-01\end{array}$ & $\begin{array}{l}1.0745 \\
1.1660 \\
1.0248 \\
1.0007 \\
1.1075 \\
0.8355 \\
1.1035 \\
0.9692\end{array}$ \\
\hline \multicolumn{7}{|c|}{ Central Reactivity Werthe linheurs/kal } \\
\hline $\begin{array}{l}U-235 \\
U-238 \\
U-233 \\
P U-239 \\
B-10 \\
T A-181 \\
M O \\
M N-55 \\
F E \\
C R \\
N I \\
N A-23 \\
\text { AL-27 } \\
O-16 \\
C\end{array}$ & $\begin{array}{r}2.160 E+02 \\
-1.303 E+01 \\
4.457 E+02 \\
4.109 E+02 \\
-3.360 E+03 \\
-5.091 E+01 \\
-2.498 E+01 \\
-1.540 E+01 \\
-1.431 E+01 \\
-1.537 E+01 \\
-1.921 E+01 \\
-1.431 E+01 \\
-1.742 E+01 \\
-2.556 E+01 \\
-3.329 E+01\end{array}$ & $\begin{array}{l}2.44 \\
3.03 \\
1.31 \\
2.39 \\
2.78 \\
3.06 \\
3.92 \\
5.56 \\
5.88 \\
5.88 \\
4.17 \\
4.29 \\
10.00 \\
17.24 \\
15.29\end{array}$ & $\begin{array}{r}2.720 E+02 \\
-1.278 E+01 \\
4.770 E+02 \\
4.456 E+02 \\
-3.115 E+03 \\
-4.899 E+01 \\
-3.124 E+01 \\
-2.180 E+01 \\
-2.054 E+01 \\
-2.278 E+01 \\
-2.725 E+01 \\
-2.908 E+01 \\
-2.607 E+01 \\
-3.102 E+01 \\
-5.323 E+01\end{array}$ & $\begin{array}{l}1.1059 \\
0.9809 \\
1.0701 \\
1.0845 \\
0.9215 \\
0.9622 \\
1.2506 \\
1.4158 \\
1.4354 \\
1.4823 \\
1.4187 \\
2.0321 \\
1.4967 \\
1.2135 \\
1.5990\end{array}$ & $\begin{array}{r}2.731 E+02 \\
-1.315 E+01 \\
4.792 E+02 \\
4.477 E+02 \\
-3.146 E+03 \\
-4.966 E+01 \\
-3.172 E+01 \\
-2.230 E+01 \\
-2.090 E+01 \\
-2.315 E+01 \\
-2.767 E+01 \\
-3.030 E+01 \\
-2.686 E+01 \\
-3.289 E+01 \\
-5.604 E+01\end{array}$ & $\begin{array}{l}1.1103 \\
1.0096 \\
1.0751 \\
1.0895 \\
0.9308 \\
0.9754 \\
1.2699 \\
1.4478 \\
1.4607 \\
1.5065 \\
1.4405 \\
2.1176 \\
1.5418 \\
1.2667 \\
1.6832\end{array}$ \\
\hline
\end{tabular}


IfBLE $B-I X$

2PR-3-12 RESULTS
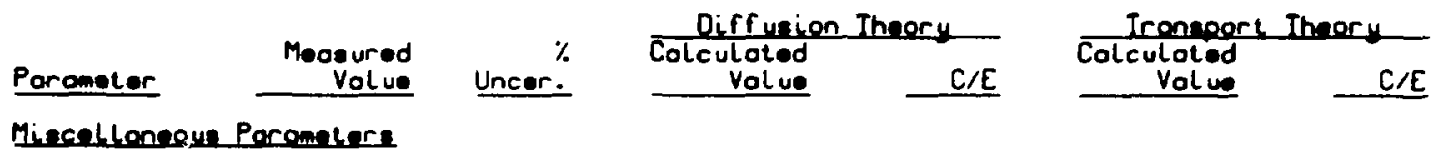

Eigenvalue $\quad 1.0005+00$

$A(/ \mathrm{S}) \quad-6.840 E+04$

0.25
2.92

9.963E-01

$-7.471 E+04$

$7.466 E+00$

Loto $(E-85)$
L $(E-3)$

$R /] H$ (E-5)

$9.993 E+00$

2. $239 E+00$

$\begin{array}{rrr}0.9963 & 1.006 \varepsilon+00 & 1.0061 \\ 1.0923 & -7.528 \varepsilon+04 & 1.1005 \\ & 7.430 E+00 & \\ & 9.871 E+00 & \\ & 2.231 E+00\end{array}$

Gentral Reaction Rale Rotios

$\begin{array}{lllllll}\text { F28/F25 } & 4.700 E-02 & 4.26 & 5.143 E-02 & 1.0943 & 5.131 E-02 & 1.0917 \\ F 24 / F 25 & 3.050 E-01 & 3.93 & 3.564 E-01 & 1.1684 & 3.561 E-01 & 1.1675 \\ F 23 / F 25 & 1.480 E+00 & 2.03 & 1.521 E+00 & 1.0274 & 1.521 E+00 & 1.0275 \\ F 49 / / 25 & 1.120 E+00 & 1.79 & 1.134 E+00 & 1.0128 & 1.134 E+00 & 1.0129 \\ 628 / F 25 & 1.230 E-01 & 4.07 & 1.188 E-01 & 0.9658 & 1.188 E-01 & 0.9657\end{array}$

Centrel Reactivily Herthe Lnhoura/kgl.

$\begin{array}{lrrrrrr}U-235 & 2.852 E+02 & 0.32 & 2.766 E+02 & 0.9697 & 2.819 E+02 & 0.9885 \\ U-238 & -1.202 E+01 & 14.93 & -1.028 E+01 & 0.8550 & -1.122 E+01 & 0.9337 \\ P U & 4.358 E+02 & 2.87 & 4.339 E+02 & 0.995 E & 4.428 E+02 & 1.0161 \\ U-233 & 4.929 E+02 & 2.60 & 1.894 E+02 & 0.9929 & 4.995 E+02 & 1.0135 \\ T A-181 & -7.315 E+01 & 3.23 & -7.209 E+01 & 0.9855 & -7.445 E+01 & 1.0178 \\ N B-93 & -4.596 E+01 & 10.00 & -4.880 E+01 & 1.0618 & -5.090 E+01 & 1.1075 \\ M O & -3.115 E+01 & 7.14 & -3.473 E+01 & 1.1149 & -3.632 E+01 & 1.1661 \\ N I & -1.964 E+01 & 18.52 & -2.309 E+01 & 1.1756 & -2.440 E+01 & 1.2422 \\ F E & -1.147 E+01 & 26.67 & -1.460 E+01 & 1.2728 & -1.574 E+01 & 1.3721 \\ \text { FL-27 } & -6.330 E+00 & 25.00 & -9.035 E+00 & 1.5536 & -1.141 E+01 & 1.8029 \\ C & 7.117 E+01 & 15.00 & 2.170 E+01 & 0.3049 & 1.837 E+01 & 0.2582\end{array}$


TABLE $B-x$

ZEBRA-2 RESULTS

\begin{tabular}{|c|c|c|}
\hline orameler & $\begin{array}{r}\text { Moosured } \\
\text { Yolue } \\
\end{array}$ & $\begin{array}{r}\% \\
\text { Uncer. }\end{array}$ \\
\hline \multicolumn{3}{|c|}{ Miecelloneous Poromelere } \\
\hline $\begin{array}{l}\text { Eigenvalue } \\
\text { A }(/ / S) \\
\text { Boto }(E-3) \\
L(E-7 S) \\
R /(H \text { (E-5) }\end{array}$ & $\begin{array}{r}1.000 E+00 \\
-2.820 E+04\end{array}$ & \\
\hline \multicolumn{3}{|c|}{ Conlral Reaclion Rate Retion } \\
\hline $\begin{array}{l}F 28 / F 25 \\
F 23 / F 25 \\
F 24 / F 25 \\
F 26 / F 25 \\
F 49 / F 25 \\
F 40 / F 25 \\
F 37 / F 25 \\
C 28 / F 25 \\
C 97 / F 25 \\
C 55 / F 25 \\
C 31 / F 25 \\
C 13 / F 25 \\
C 50 / F 25\end{array}$ & $\begin{array}{l}3.2 C 0 E-02 \\
1.453 E+00 \\
1.530 E-01 \\
9.300 E-02 \\
9.870 E-01 \\
2.370 E-01 \\
2.140 E-01 \\
1.360 E-01 \\
2.980 E-01 \\
2.600 E-02 \\
4.010 E-01 \\
1.300 E-03 \\
1.580 E+C 0\end{array}$ & $\begin{array}{r}1.56 \\
0.96 \\
10.46 \\
15.05 \\
1.01 \\
1.69 \\
0.93 \\
0.74 \\
5.03 \\
7.69 \\
9.98 \\
7.69 \\
4.43\end{array}$ \\
\hline
\end{tabular}
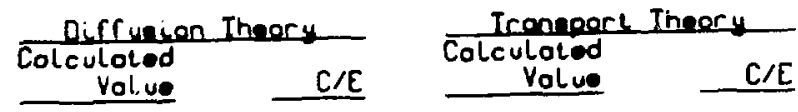

$\begin{array}{rr}9.956 E-01 & 0.9956 \\ -3.427 E+04 & 1.2154 \\ 7.388 E+00 & \\ 2.155 E+00 & \\ 2.207 E & \end{array}$

$\begin{array}{rl}9.988 E-01 & 0.9988 \\ -3.430 E+04 & 1.2164 \\ 7.375 E+00 & \\ 2.150 E+00 & \\ 2.204 E+00 & \end{array}$

Central Reactivity Horthe (inhours/kg)

$\begin{array}{lrrrrrr}U-235 & 1.397 E+02 & 1.02 & 1.514 E+02 & 1.0838 & 1.526 E+02 & 1.0926 \\ U-238 & -1.069 E+01 & 5.66 & -1.085 E+01 & 1.0150 & -1.104 E+01 & 1.0332 \\ P U-239 & 1.954 E+02 & 1.23 & 2.133 E+02 & 1.0918 & 2.151 E+02 & 1.1008 \\ P U-240 & 4.399 E+01 & 18.18 & 2.998 E+01 & 0.6818 & 3.008 E+01 & 0.6837 \\ B-10 & -4.505 E+03 & 3.19 & -3.896 E+03 & 0.8645 & -3.934 E+03 & 0.8730 \\ B & -8.692 E+02 & 5.00 & -7.127 E+02 & 0.8015 & -7.200 E+02 & 0.8097 \\ L 1-6 & -3.112 E+03 & 5.13 & -2.567 E+03 & 0.8248 & -2.592 E+03 & 0.8330 \\ L I & -2.909 E+02 & 9.52 & -1.846 E+02 & 0.6345 & -1.872 E+02 & 0.6435 \\ T A-181 & -6.154 E+01 & 1.72 & -7.626 E+01 & 1.2392 & -7.709 E+01 & 1.2527 \\ H F & -5.620 E+01 & 1.91 & -6.547 E+01 & 1.1650 & -E .619 E+01 & 1.1778 \\ A U-197 & -3.655 E+01 & 6.67 & -5.404 E+01 & 1.4786 & -5.464 E+01 & 1.1951 \\ C U & -1.360 E+01 & 5.56 & -2.016 E+01 & 1.4825 & -2.048 E+01 & 1.5055 \\ F E & -5.157 E+00 & 16.67 & -7.185 E+00 & 1.3932 & -7.361 E+00 & 1.1273 \\ C R & -5.539 E+00 & 16.67 & -9.198 E+00 & 1.6606 & -9.387 E+00 & 1.6947 \\ N 1 & -9.811 E+00 & 8.33 & -9.807 E+00 & 0.9996 & -1.000 E+01 & 1.0194 \\ M N-55 & -7.427 E+00 & 11.76 & -1.472 E+01 & 1.9824 & -1.497 E+01 & 2.0156 \\ N A-23 & 2.923 E+00 & 71.43 & 8.018 E+00 & 2.7429 & 7.864 E+00 & 2.6904 \\ \text { AL-27 } & -4.803 E+00 & 37.04 & -6.493 E+00 & 1.3519 & -6.715 E+00 & 1.3980 \\ C & 1.240 E+01 & 16.13 & 7.711 E+00 & 0.6218 & 7.402 E+00 & 0.5969 \\ M-1 & 7.144 E+03 & 6.67 & 7.454 E+03 & 1.0433 & 7.497 E+03 & 1.0495\end{array}$


IAELE B-XI

ZPPR-Z RESULTS
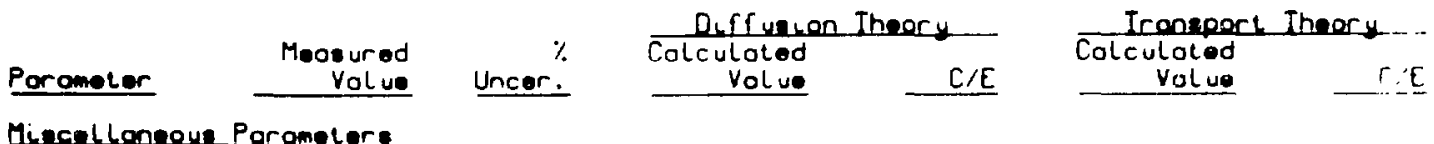

\begin{tabular}{|c|c|c|c|}
\hline 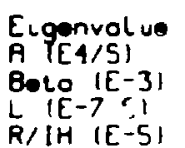 & $1.000 E+00$ & 0.06 & $\begin{array}{r}9.817 \mathrm{E}-01 \\
-7.352 \mathrm{E}-01 \\
3.402 \mathrm{E}+00 \\
4.627 \mathrm{E}+00 \\
1.040 \mathrm{O}+00\end{array}$ \\
\hline
\end{tabular}

0.9817

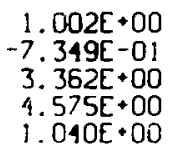

1.0017

Gentral Reaction Rote Rolver

$\begin{array}{lllllll}F 23 / F 25 & 1.446 E+00 & 1.52 & 1.433 E-00 & 0.9913 & 1.432 E+00 & 0.9906 \\ F 24 / F 25 & 1.492 E-01 & 1.54 & 1.751 E-01 & 1.1734 & 1.730 E-01 & 1.1593 \\ F 26 / F 25 & 4.430 E-02 & 1.58 & 5.543 E-02 & 1.2512 & 5.469 E-02 & 1.2344 \\ F 28 / F 25 & 2.010 E-02 & 1.99 & 2.266 E-02 & 1.1271 & 2.232 E-02 & 1.1162 \\ F 49 / F 25 & 9.372 E-01 & 1.52 & 9.392 E-01 & 1.0022 & 9.371 E-01 & 0.9599 \\ F 40 / F 25 & 1.704 E-01 & 1.53 & 1.964 E-01 & 1.1524 & 1.942 E-01 & 1.1396\end{array}$

Cencrab Reacliybly Worthe Ientioure/kgl

$\begin{array}{lrrrrrr}P U-239 & 1.202 E+02 & 0.67 & 1.340 E+02 & 1.1147 & 1.323 E+02 & 1.1005 \\ P U-241 & 1.631 E+02 & 12.66 & 1.975 E+02 & 1.2110 & 1.958 E+02 & 1.2004 \\ U-235 & 9.014 E+01 & 2.30 & 1.070 E+02 & 1.1873 & 1.058 E+02 & 1.1739 \\ C & -1.141 E+01 & 6.90 & -1.388 E+01 & 1.2165 & -1.521 E+01 & 1.3331 \\ N R-23 & -5.178 E+00 & 4.78 & -6.041 E+00 & 1.1666 & -5.520 E+00 & 1.2533 \\ I R-181 & -5.108 E+01 & 1.98 & -4.418 E+C 1 & 0.8650 & -4.452 E+01 & 0.8715 \\ B-10 & -2.269 E+03 & 1.83 & -2.178 E+03 & 0.9598 & -2.189 E+03 & 0.9648 \\ F E & -3.157 E+00 & 2.59 & -3.705 E+00 & 1.1735 & -3.815 E+00 & 1.2084 \\ C R & -3.399 E+00 & 6.90 & -5.089 E+00 & 1.4972 & -5.220 E+00 & 1.5359 \\ N 1 & -4.766 E+00 & 3.45 & -5.642 E+00 & 1.1838 & -5.807 E+00 & 1.2185 \\ R L-27 & -6.023 E+00 & 8.75 & -7.082 E+00 & 1.1759 & -7.417 E+00 & 1.2315 \\ M N-55 & -7.654 E+00 & 4.35 & -8.199 E+00 & 1.0713 & -8.431 E+00 & 1.10 .5 \\ H & -1.099 E+01 & 0.46 & -1.560 E+01 & 1.1195 & -1.57 B E+01 & 1.4355 \\ M O & -1.295 E+01 & 4.25 & -1.443 E+01 & 1.1140 & -1.460 E+01 & 1.1275 \\ N B-93 & -1.756 E+01 & 3.74 & -2.2285+01 & 1.2687 & -2.250 E+01 & 1.2815\end{array}$


IABLE $\theta-x I 1$

2PR-6-7 RESULTS

\begin{tabular}{|c|c|c|c|c|c|c|}
\hline \multirow[b]{2}{*}{ Porameter } & \multirow[b]{2}{*}{$\begin{array}{r}\text { Measured } \\
\text { Volue } \\
\end{array}$} & \multirow[b]{2}{*}{ Uncer: } & \multicolumn{2}{|c|}{ OLfrueion Iheory } & \multicolumn{2}{|c|}{ Iranupere Theory } \\
\hline & & & $\begin{array}{r}\text { Colculoled } \\
\text { volue } \\
\end{array}$ & $\mathrm{C} / \mathrm{E}$ & $\begin{array}{r}\text { Colculoted } \\
\text { Yolue }\end{array}$ & C/E \\
\hline \multicolumn{7}{|c|}{ Misaceloneeve Poramelers } \\
\hline $\begin{array}{l}\text { Eigenval ve } \\
\text { A YE4/S) } \\
\text { Bela (E-3) } \\
L(E-7 \text { S) } \\
R / I H \text { (E-5) }\end{array}$ & $1.000 E+00$ & 0.10 & $\begin{array}{r}9.824 E-01 \\
-6.972 E-01 \\
3.450 E+00 \\
4.949 E+00 \\
1.051 E+00\end{array}$ & 0.9824 & $\begin{array}{r}9.841 E-01 \\
-6.960 E-01 \\
3.442 E+00 \\
4.945 E+00 \\
1.049 t+00\end{array}$ & 0.9841 \\
\hline \multicolumn{7}{|c|}{ Centrak Reoction Rote Roltes } \\
\hline $\begin{array}{l}C 28 / \Gamma 49 \\
F 28 / F 49 \\
F 25 / F 49\end{array}$ & $\begin{array}{l}1.432 E-01 \\
2.406 E-02 \\
1.049 E+00\end{array}$ & $\begin{array}{l}2.00 \\
2.00 \\
2.00\end{array}$ & $\begin{array}{l}1.537 \mathrm{E}-01 \\
2.129 \mathrm{E}-02 \\
1.066 \mathrm{E}+00\end{array}$ & $\begin{array}{l}1.0733 \\
1.0096 \\
1.0156\end{array}$ & $\begin{array}{l}1.537 E-01 \\
2.426 E-02 \\
1.066 E+00\end{array}$ & $\begin{array}{l}1.0735 \\
1.0090 \\
1.0157\end{array}$ \\
\hline \multicolumn{7}{|c|}{ Central Reaclively Hor the (unhoure/kg) } \\
\hline $\begin{array}{l}P U-239 \\
U-235 \\
U-238 \\
U-10 \\
N A-23 \\
T A-181 \\
C \\
\text { AL-27 } \\
\text { FE } \\
N ! \\
C R \\
M O\end{array}$ & $\begin{array}{r}1.586 E+02 \\
1.332 E+02 \\
-1.022 E+01 \\
-2.735 E+03 \\
-6.648 E+00 \\
-4.307 E+01 \\
-1.220 E+01 \\
-6.718 E+00 \\
-4.270 E+00 \\
-6.466 E+00 \\
-1.538 E+00 \\
-1.539 E+01\end{array}$ & $\begin{array}{r}1.06 \\
1.51 \\
4.19 \\
2.15 \\
5.16 \\
10.08 \\
1.72 \\
2.50 \\
3.63 \\
2.86 \\
8.15 \\
0.68\end{array}$ & $\begin{array}{r}1.850 E+02 \\
1.478 E+02 \\
-1.053 E+01 \\
-3.037 E+03 \\
-8.210 E+00 \\
-6.181 E+01 \\
-1.866 E+01 \\
-3.711 E+00 \\
-5.128 E+00 \\
-7.806 E+00 \\
-7.019 E+00 \\
-2.011 E+01\end{array}$ & $\begin{array}{l}1.1668 \\
1.1097 \\
1.0299 \\
1.1105 \\
1.2350 \\
1.4358 \\
1.5296 \\
1.4456 \\
1.2010 \\
1.2072 \\
1.5532 \\
1.3070\end{array}$ & $\begin{array}{r}1.861 E+02 \\
1.486 E+02 \\
-1.062 E+01 \\
-3.056 E+03 \\
-8.326 E+00 \\
-6.225 E+01 \\
-1.892 E+01 \\
-9.811 E+00 \\
-5.183 E+00 \\
-7.880 E+00 \\
-7.116 E+00 \\
-2.026 E+01\end{array}$ & $\begin{array}{l}1.1731 \\
1.1157 \\
1.0388 \\
1.1175 \\
1.2525 \\
1.4453 \\
1.5505 \\
1.4609 \\
1.2139 \\
1.2187 \\
1.5682 \\
1.3167\end{array}$ \\
\hline
\end{tabular}


TABLE B-XIII

ZPR-3-56E RESULTS

\begin{tabular}{|c|c|}
\hline - Omoter & $\begin{array}{l}\text { Meosured } \\
\text { Volue }\end{array}$ \\
\hline \multicolumn{2}{|c|}{ Miscallonnous Paramoters } \\
\hline $\begin{array}{l}\text { Eigenvol ue } \\
A \text { (E4/S) } \\
\text { Belo (E-3) } \\
L / E-7 S) \\
R / I H(E-S)\end{array}$ & $1.000 E+00$ \\
\hline
\end{tabular}

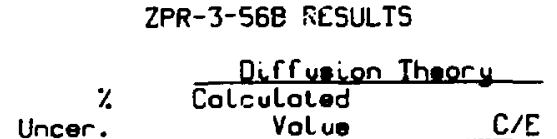

Central Ronction Rote Rotios

$\begin{array}{lllllll}F 23 / F 25 & 1.478 E+00 & 1.01 & 1.463 E+00 & 0.9897 & 1.463 E+00 & 0.9895 \\ F 24 / F 25 & 1.950 E-01 & 1.03 & 3.260 E-01 & 1.1589 & 2.253 E-01 & 1.1553 \\ F 26 / F 25 & 6.390 E-02 & 0.94 & 7.293 E-02 & 1.1414 & 7.268 E-02 & 1.1373 \\ F 28 / F 25 & 3.080 E-02 & 0.97 & 3.083 E-02 & 1.0010 & 3.071 E-02 & 0.9972 \\ F 49 / F 25 & 1.028 E+00 & 0.97 & 9.933 E-01 & 0.9662 & 9.927 E-01 & 0.5656 \\ F 40 / F 25 & 2.820 E-01 & 1.06 & 2.492 E-01 & 0.8837 & 2.485 E-01 & 0.8811\end{array}$

Centrol Reoctivily Herths linhours $/ \mathrm{kgl}$

$\begin{array}{lrrrrrr}U-235 & 2.948 E+02 & 2.81 & 3.129 E+02 & 1.0613 & 3.120 E+02 & 1.0582 \\ U-238 & -1.840 E+01 & 4.44 & -2.081 E+01 & 1.1310 & -2.1114 E+01 & 1.1487 \\ P U-239 & 3.709 E+02 & 1.99 & 4.244 E+02 & 1.1444 & 4.232 E+02 & 1.1410 \\ C & -2.493 E+01 & 10.06 & -4.469 E+01 & 1.7928 & -4.618 E+01 & 1.8523 \\ N A-23 & -6.930 E+00 & 46.98 & -1.976 E+01 & 2.2127 & -2.043 E+01 & 2.2878 \\ C R & -1.275 E+01 & 3.75 & -1.684 E+01 & 1.3211 & -1.709 E+01 & 1.3407 \\ F E & -1.230 E+01 & 3.74 & -1.272 E+01 & 1.0345 & -1.235 E+01 & 1.0531 \\ N I & -1.681 E+01 & 3.32 & -2.037 E+01 & 1.2119 & -2.065 E+01 & 1.2287 \\ B-10 & -7.000 E+03 & 1.39 & -6.259 E+03 & 0.8911 & -6.277 E+03 & 0.8967 \\ T A-1 B 1 & -1.200 E+02 & 3.71 & -1.163 E+02 & 0.9696 & -1.169 E+02 & 0.9738\end{array}$

TABLE B-XIV

ZPR-6-6R RESULIS

\begin{tabular}{|c|c|}
\hline Poromeler & $\begin{array}{r}\text { Moosured } \\
\text { Vol ue } \\
\end{array}$ \\
\hline \multicolumn{2}{|c|}{ Mincelloneous Porometers } \\
\hline $\begin{array}{l}\text { Eigenval ue } \\
\text { A }(E 4 / S) \\
\text { Eoto }(E-3) \\
L \text { (E-7 S) } \\
R / I H(E-5)\end{array}$ & $1.000 E+00$ \\
\hline
\end{tabular}
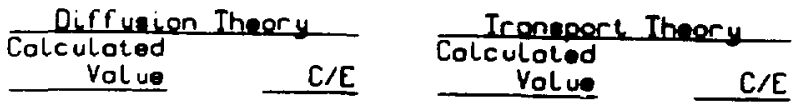

Central Reaction Rote Rotion

$\begin{array}{lllllll}\text { F28/F25 } & 2.446 E-02 & 2.98 & 2.411 E-02 & 0.9858 & 2.410 E-02 & 0.9855 \\ \text { C26/F25 } & 1.393 E-01 & 2.94 & 1.443 E-01 & 1.0362 & 1.44 \mathrm{E}-01 & 1.0363\end{array}$

Centrol Reeclivity Worthe linhoure/kgl

$\begin{array}{lrrrrrr}P U-239 & 5.630 E+01 & 3.48 & 6.132 E+01 & 1.0891 & 6.161 E+01 & 1.0943 \\ U-235 & 4.050 E+01 & 1.16 & 4.511 E+01 & 1.1138 & 4.532 E+01 & 1.1191 \\ U-230 & -3.343 E+00 & 0.27 & -3.898 E+00 & 1.1662 & -3.924 E+00 & 1.1739 \\ M A-23 & 6.535 E-02 & 25.60 & 1.281 E-02 & 0.1960 & -5.340 E-03 & 0.0817 \\ B-10 & -1.250 E+03 & 4.40 & -1.174 E+03 & 0.9389 & -1.180 E+03 & 0.9436 \\ T R-181 & -1.250 E+01 & 0.08 & -2.161 E+01 & 1.7285 & -2.172 E+01 & 1.7377 \\ C & 3.865 E+00 & 0.49 & 2.390 E+00 & 0.6184 & 2.363 E+00 & 0.6114\end{array}$


IABLE B-XV

SNEAK-7A RESULTS

\begin{tabular}{|c|c|c|c|c|c|c|}
\hline \multirow[b]{2}{*}{ Porometer } & \multirow[b]{2}{*}{$\begin{array}{r}\text { Moosured } \\
\text { Yolue } \\
\end{array}$} & \multirow[b]{2}{*}{ Uncer." } & \multicolumn{2}{|c|}{ Diffunion Theory } & \multicolumn{2}{|c|}{ Troniport Thoory } \\
\hline & & & $\begin{array}{r}\text { Colculated } \\
\text { Yolve }\end{array}$ & $\mathrm{C} / \mathrm{E}$ & $\begin{array}{r}\text { Colculoted } \\
\text { Yolue }\end{array}$ & $C / E$ \\
\hline \multicolumn{7}{|c|}{ Minenllomeus Porometere } \\
\hline $\begin{array}{l}\text { Eigenvolue } \\
\text { A (E4/S) } \\
\text { Boto }(E-3) \\
L(E-7 S) \\
R / I H(E-5)\end{array}$ & $1.000 E+00$ & 0.10 & $\begin{array}{r}9.944 E-01 \\
-2.137 E+00 \\
3.650 E+00 \\
1.708 E+00 \\
1.100 E+00\end{array}$ & 0.9944 & $\begin{array}{r}1.006 E+\infty 0 \\
-2.128 E+00 \\
3.595 E+00 \\
1.690 E+00 \\
1.089 E+\infty 0\end{array}$ & 1.0056 \\
\hline
\end{tabular}

Central Reaction Rote Rotion

$\begin{array}{lllllll}\text { F28/F25 } & 4.480 E-02 & 3.00 & 4.358 E-02 & 0.9727 & 4.375 E-22 & 0.9767 \\ F 4 J / F 25 & 1.016 E+00 & 3.00 & 9.981 E-01 & 0.9824 & 9.992 E-01 & 0.9835 \\ \text { C28/F25 } & 1.376 E-01 & 3.00 & 1.365 E-01 & 0.9923 & 1.364 E-01 & 0.9914\end{array}$

Central Regetivity Herthe ( $\mathbf{k g}$ )

\begin{tabular}{|c|c|c|c|c|c|c|}
\hline $\begin{array}{l}U-235 \\
U-238 \\
P U-239 \\
P U-240 \\
B-10 \\
T A-181 \\
E U 203 \\
F E\end{array}$ & $\begin{array}{r}2.528 E+00 \\
-1.385 E-01 \\
3.434 E+00 \\
8.587 E-01 \\
-6.696 E+01 \\
-1.755 E+00 \\
-4.053 E+00 \\
-1.137 E-01\end{array}$ & $\begin{array}{r}3.00 \\
3.00 \\
3.00 \\
8.00 \\
2.00 \\
2.00 \\
2.00 \\
10.00\end{array}$ & $\begin{array}{r}2.180 E+00 \\
-1.393 E-01 \\
3.478 E+00 \\
6.856 E-01 \\
-5.866 E+01 \\
-1.117 E+00 \\
-4.041 E+00 \\
-1.008 E-01\end{array}$ & $\begin{array}{l}0.9812 \\
1.0061 \\
1.0128 \\
0.7984 \\
0.8760 \\
0.6365 \\
0.9969 \\
0.8863\end{array}$ & $\begin{array}{r}2.573 E+00 \\
-1.509 E-01 \\
3.614 E+00 \\
7.085 E-01 \\
-6.084 E+01 \\
-1.164 E+00 \\
-4.193 E+00 \\
-1.140 E-01\end{array}$ & $\begin{array}{l}1.0176 \\
1.0898 \\
1.0525 \\
0.8251 \\
0.9086 \\
0.6633 \\
1.0346 \\
1.0024\end{array}$ \\
\hline
\end{tabular}

IABLE B-XVI

SNEFK-7B RESULTS

\section{Porometer Mincelloneus Porometere}

Eigenval ue $\quad 1.000 \mathrm{E}+00$

\section{A $(E 4 / S)$}

Belo (E-3)

$L(E-7$ s)

$R /$ IH (E-5)

\section{Centrol Reaction Rote Rotios}

$\begin{array}{lll}\text { F28/F25 } & 3.300 E-02 & 2.00 \\ \text { F49/F25 } & 1.012 E+00 & 2.00 \\ \text { C28/F25 } & 1.312 E-01 & 3.00\end{array}$

Centrol Reactivity Horthe (Skg)

$\begin{array}{lrr}U-235 & 1.278 E+00 & 2.00 \\ U-238 & -7.336 E-02 & 2.00 \\ P U-239 & 1.651 E+00 & 2.00 \\ P U-240 & 3.275 E-01 & 10.00 \\ B-10 & -2.369 E+01 & 2.00 \\ \text { TA-18! } & -4.946 E-01 & 2.00 \\ \text { EU203 } & -1.264 E+00 & 3.00 \\ \text { FE } & -6.491 E-02 & 5.00\end{array}$

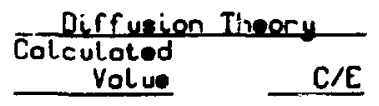

1.0001

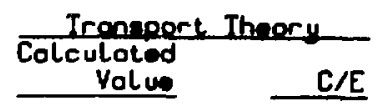

i. $005 E \cdot 00$

$-2.638 E+00$

4.173E+00

$1.582[+00$

$1.200 E+00$

1.0048

$4.191 \mathrm{E}+00$

(1)

3. $416 \mathrm{E}-02$

$1.022 E+00$

1.0351

1.0100

3.112E-02

$1.022 E+00$

1.0341

$1.348 E-01$

1.0276

i. $349 \mathrm{C}-01$

1.0279

$1.287 E+00$
$-7.611 E-02$
$1.776 E+00$
$3.012 E-01$
$-2.095 E+01$
$-3.675 E-01$
$-1.358 E+00$
$-7.043 E-02$

1.0073

$1.302 E+\infty 0$

1.0185

1. 0100

$-7.806 E-02$

1.0361

$1.796 E+00$

1.0677

0.9197

0.8842

$3.029 E-41$

0.9250

$-2.123 E+01$

$0.7430-3.733 E-01$

0.8962

1.0745

1.0851

$-1.377 E+00$

0.7547

i. 0096

$-7.212 E-02$

1.1157 
TABLE 8-XVII

2PR-9-31 RESLLIS

\begin{tabular}{|c|c|c|c|c|c|c|}
\hline \multirow[b]{2}{*}{ Poromeler } & \multirow[b]{2}{*}{$\begin{array}{r}\text { Meosured } \\
\text { Yolue } \\
\end{array}$} & \multirow[b]{2}{*}{ Uncer. } & \multicolumn{2}{|c|}{ Diffurion Theory } & \multicolumn{2}{|c|}{ Ironport Theor: } \\
\hline & & & $\begin{array}{r}\text { Colculoted } \\
\text { Volue } \\
\end{array}$ & C/E & $\begin{array}{r}\text { Colculated } \\
\text { Volue }\end{array}$ & $C / E$ \\
\hline \multicolumn{7}{|c|}{ Misenltomenus Porometers } \\
\hline $\begin{array}{l}\text { Eigenvolue } \\
\text { A (EA/S) } \\
\text { Selo (E-3) } \\
L \text { (E-7S) } \\
R /(H \text { (E-S) }\end{array}$ & $1.000 E+00$ & 0.15 & $\begin{array}{r}9.908 E-01 \\
-1.255 E+00 \\
3.748 E+00 \\
2.986 E+00 \\
1.094 E+00\end{array}$ & 0.9908 & $\begin{array}{r}9.955 E-01 \\
-1.262 E+00 \\
3.735 E+00 \\
2.960 E+00 \\
1.091 E+00\end{array}$ & 0.9955 \\
\hline
\end{tabular}

Contral Reoction Rote Hotios

$\begin{array}{lllllll}F 28 / \mathrm{F} 49 & 3.000 E-02 & 1.33 & 2.992 E-02 & 0.9973 & 2.985 E-02 & 0.9949 \\ F 25 / F 49 & 1.052 E+00 & 1.32 & 9.80 \mathrm{E}-01 & 0.9404 & 9.892 E-01 & 0.9407 \\ \mathrm{C} 28 / \mathrm{F} 49 & 1.274 E-01 & 1.46 & 1.317 E-01 & 1.0311 & 1.318 E-01 & 1.0349\end{array}$

Central Reaclixily Horthe linhours/kgl

$\begin{array}{lrrrrrr}P U-239 & 2.867 E+02 & 1.05 & 3.317 E+02 & 1.1568 & 3.328 E+02 & 1.1607 \\ P U-240 & 4.340 E+01 & 2.13 & 5.111 E+01 & 1.1815 & 5.123 E+01 & 1.1801 \\ P U-241 & 3.951 E+02 & 1.09 & 1.438 E+02 & 1.1231 & 4.455 E+02 & 1.1275 \\ U-233 & 3.473 E+02 & 1.36 & 4.159 E+02 & 1.1975 & 4.175 E+02 & 1.2020 \\ U-235 & 2.139 E+02 & 0.97 & 2.433 E+02 & 1.1374 & 2.441 E+02 & 1.1413 \\ U-238 & -1.445 E+01 & 3.82 & -1.466 E+01 & 1.0147 & -1.490 E+01 & 1.0308 \\ B-10 & -4.023 E+03 & 1.69 & -4.110 E+03 & 1.0217 & -4.138 E+03 & 1.0287 \\ L 1-6 & -2.842 E+03 & 1.86 & -3.145 E+03 & 1.1057 & -3.167 E+03 & 1.1145 \\ C & -3.610 E+01 & 2.57 & -4.740 E+01 & 1.3129 & -4.848 E+01 & 1.3430 \\ N A-23 & -1.552 E+01 & 7.22 & -2.045 E+01 & 1.3175 & -2.092 E+01 & 1.3477 \\ \text { PL-27 } & -1.557 E+01 & 1.09 & -2.179 E+01 & 1.3997 & -2.215 E+01 & 1.1227\end{array}$


[fBL[ $B-x y 1]]$

EZEECL 70-GROUP TRANSPORT THEORT SPECTRA

\begin{tabular}{|c|c|c|c|c|c|c|c|}
\hline rano & $\begin{array}{r}\text { Enargy } \\
\text { Boundory } \\
\text { InYII }\end{array}$ & $\begin{array}{r}\text { Lethorgy } \\
\text { Hiddh }\end{array}$ & $\begin{array}{r}\text { Reoctor } \\
\text { Fiseion } \\
\text { Frochionn }\end{array}$ & $\begin{array}{r}\text { Centrol } \\
\text { flux } \\
\left.\ln / \mathrm{an}^{2} / \mathrm{l}\right) \\
\end{array}$ & $\begin{array}{r}\text { Control } \\
\text { Ad jount } \\
\text { irhotniel }\end{array}$ & $\begin{array}{r}\text { Edge } \\
f \operatorname{lux} \\
\ln \left(\cos ^{2} / \Omega\right) \\
\end{array}$ & $\begin{array}{r}\text { Edge } \\
\text { Adjound } \\
\text { Icholotel }\end{array}$ \\
\hline $\begin{array}{l}1 \\
2 \\
3 \\
4 \\
5 \\
6 \\
7 \\
8 \\
9 \\
10 \\
11 \\
12 \\
13 \\
14 \\
15 \\
16 \\
17 \\
18 \\
19 \\
20 \\
21 \\
22 \\
23 \\
24 \\
25 \\
26 \\
27 \\
28 \\
29 \\
30 \\
31 \\
32 \\
33 \\
34 \\
35 \\
36 \\
37 \\
38 \\
39 \\
40 \\
41 \\
42 \\
43 \\
44 \\
45 \\
46 \\
47 \\
48 \\
49 \\
50 \\
51 \\
52 \\
53 \\
54 \\
55 \\
56 \\
57 \\
58 \\
59 \\
60 \\
61 \\
62 \\
63 \\
64 \\
65 \\
66 \\
69 \\
69 \\
70\end{array}$ & 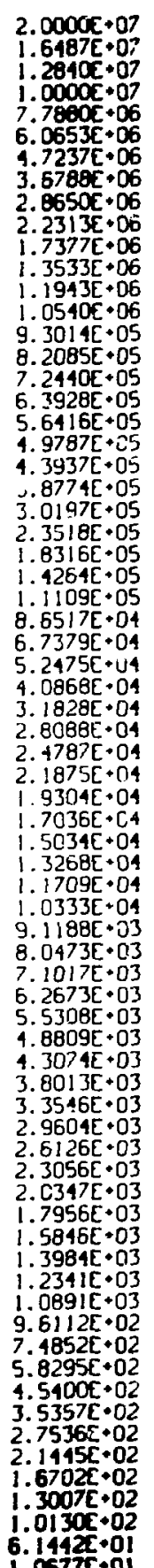 & $\begin{array}{l}1.9315 E-01 \\
2.5000 E-01 \\
2.5000 E-01 \\
2.5000 E-01 \\
2.5000 E-01 \\
2.5000 E-01 \\
2.5000 E-01 \\
2.5000 E-01 \\
2.5000 E-01 \\
2.5000 E-01 \\
2.5000 E-01 \\
1.2500 E-01 \\
1.25005-01 \\
1.2500 E-01 \\
1.2500 E-01 \\
1.2500 E-01 \\
1.2500 E-01 \\
1.2500 E-01 \\
1.2500 E-01 \\
1.2500 E-01 \\
1.2500 E-01 \\
2.5000 E-01 \\
2.5000 E-01 \\
2.5000 E-01 \\
2.5000 E-01 \\
2.5000 E-01 \\
2.5000 E-01 \\
2.5000 E-01 \\
2.5000 E-01 \\
2.5000 E-0 . \\
2.5000 E-01 \\
1.2500 E-01 \\
1.2500 E \\
1.2500 E-01 \\
1.2500 E-01 \\
1.2500 E-01 \\
1.2500 E-01 \\
1.2500 E-01 \\
1.2500 E-01 \\
1.2497 E-01 \\
1.2503 E-01 \\
1.2500 E-01 \\
1.2500 E-01 \\
1.2500 E-01 \\
1.2500 E-01 \\
1.2500 E-01 \\
1.2500 E-01 \\
1.2500 E-01 \\
1.2500 E-01 \\
1.2500 E-01 \\
1.2500 E-01 \\
1.2500 E-01 \\
1.2500 E-01 \\
1.2500 E-01 \\
1.2500 E-01 \\
1.2500 E-01 \\
1.2500 E-01 \\
1.2500 E-01 \\
1.2500 E-01 \\
2.5000 E-01 \\
2.5000 E-01 \\
2.5000 E-01 \\
2.5000 E-01 \\
2.5000 E-01 \\
2.5000 E-01 \\
2.5000 E-01 \\
2.5000 E-01 \\
2.5000 E-01 \\
5.0000 E-01 \\
1.7500 E-00\end{array}$ & 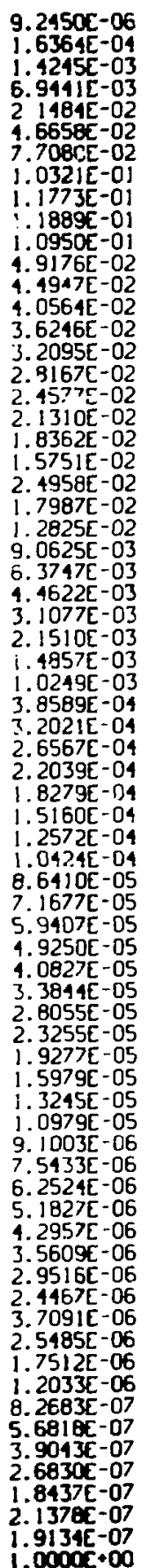 & $\begin{array}{l}\text { 6.7582E-06 } \\
1.2057 E-04 \\
1.0751 E-03 \\
5.3239 E-03 \\
1.6057 E-02 \\
3.3966 E-02 \\
5.6186 E-02 \\
7.5715 E-02 \\
8.9152 E-02 \\
9.4681 E-02 \\
9.4610 E-02 \\
4.6015 E-02 \\
4.4910 E-02 \\
4.3305 E-02 \\
4.1262 E-02 \\
3.8831 E-02 \\
3.6521 E-02 \\
3.3835 E-02 \\
3.1115 E-02 \\
2.8378 E-02 \\
2.5542 E-02 \\
4.3013 E-02 \\
3.3106 E-02 \\
2.4922 E-02 \\
1.8649 E-02 \\
1.3116 E-02 \\
9.6789 E-03 \\
6.7260 E-03 \\
4.5362 E-03 \\
3.1591 E-03 \\
2.1395 E-03 \\
7.8171 E-04 \\
6.3431 E-04 \\
5.0582 E-04 \\
4.1001 E-04 \\
3.3361 E-04 \\
2.7434 E-04 \\
2.2681 E-04 \\
1.8204 E-04 \\
1.4650 E-04 \\
1.1500 E-04 \\
8.6862 E-05 \\
6.7152 E-05 \\
5.4265 E-05 \\
4.1790 E-05 \\
3.3088 E-05 \\
2.7207 E-05 \\
2.1336 E-05 \\
1.6640 E-05 \\
1.3193 E-05 \\
1.1324 E-05 \\
8.7692 E-06 \\
6.8545 E-06 \\
5.4646 E-06 \\
4.3804 E-06 \\
3.8110 E-06 \\
2.6182 E-06 \\
2.0633 E-06 \\
1.5118 E-06 \\
2.5581 E-06 \\
1.7582 E-06 \\
8.3246 E-07 \\
7.6074 E-07 \\
4.1406 E-07 \\
1.7454 E-07 \\
1.6293 E-07 \\
1.0430 E-07 \\
5.9515 E-08 \\
3.9674 E-08 \\
4.1138 E-00 \\
1.0000 E-00\end{array}$ & 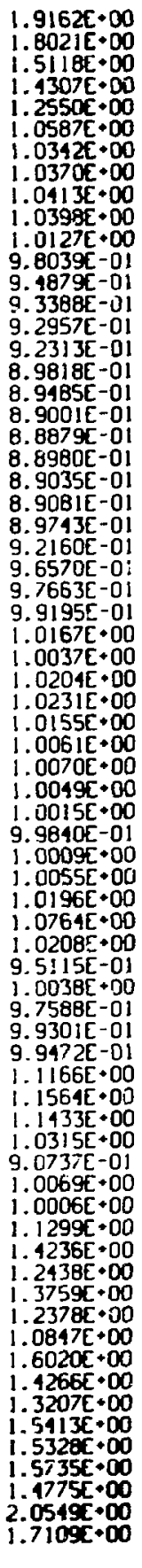 & 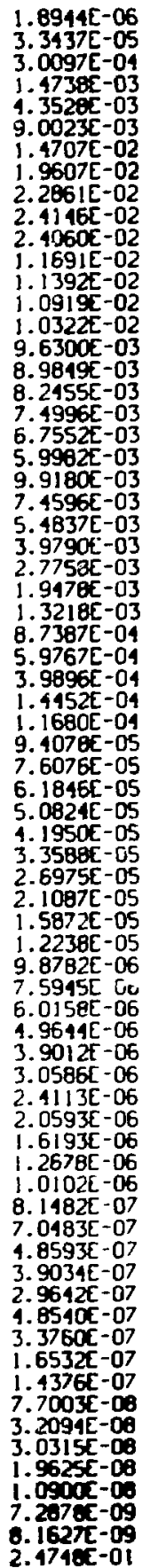 & 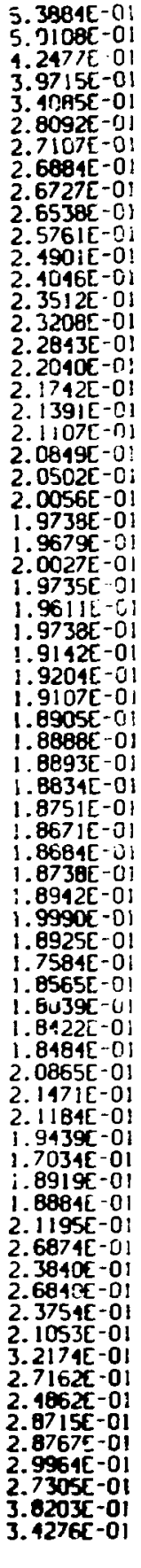 \\
\hline
\end{tabular}


TPBLE $8-x \mid X$

\begin{tabular}{|c|c|c|c|c|c|c|c|}
\hline - ovo & $\begin{array}{r}\text { Energy } \\
\text { Boundory } \\
\text { inty) }\end{array}$ & $\begin{array}{r}\text { Lothergy } \\
\text { Hedih }\end{array}$ & $\begin{array}{r}\text { Reoclor } \\
\text { Flescon } \\
\text { Fractions }\end{array}$ & $\begin{array}{r}\text { Contral } \\
\text { Flux } \\
\ln / \mathrm{em}^{2} / \mathrm{s} l \\
\end{array}$ & $\begin{array}{r}\text { Cenerol } \\
\text { Ad joune } \\
\text { Irholnol } \\
\end{array}$ & $\begin{array}{r}\text { Ldge } \\
f(u x \\
\left.\ln / c e^{2} / c\right) \\
\end{array}$ & $\begin{array}{r}\text { Edgo } \\
\text { Ad jount } \\
\text { Irhotols } \\
\end{array}$ \\
\hline $\begin{array}{l}1 \\
2 \\
3 \\
4 \\
5 \\
6 \\
7 \\
8 \\
9 \\
10 \\
11 \\
12 \\
13 \\
14 \\
15 \\
16 \\
17 \\
18 \\
19 \\
7 \\
22 \\
22 \\
23 \\
24\end{array}$ & 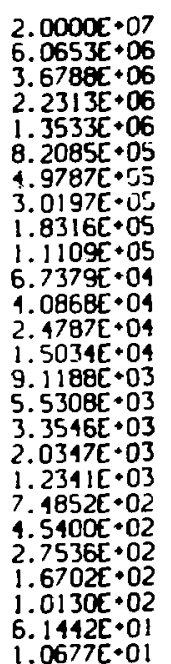 & $\begin{array}{l}\text { 1. } 1931 E-00 \\
5.0000 E-01 \\
5.0000 E-01 \\
5.0000 E-01 \\
5.0000 E-01 \\
5.0000 E-01 \\
5.0000 E-01 \\
5.0000 E-01 \\
5.0000 E-01 \\
5.0000 E-01 \\
5.0000 E-01 \\
5.0000 E-01 \\
5.0000 E-01 \\
5.0000 E-01 \\
5.0000 E-01 \\
5.0000 E-01 \\
5.0000 E-01 \\
5.0000 E-01 \\
5.0000 E-01 \\
5.0000 E-01 \\
5.0000 E-01 \\
5.0000 E-01 \\
5.0000 E-01 \\
5.0000 E-01 \\
1.7500 E-00\end{array}$ & $\begin{array}{l}3.0026 E-02 \\
1.2374 E-01 \\
2.2095 E-01 \\
2.2839 E-01 \\
1.7093 E-01 \\
1.0615 E-01 \\
5.9072 E-02 \\
3.0812 E-02 \\
1.5437 E-02 \\
7.5699 E-03 \\
3.6368 E-03 \\
1.7310 E-03 \\
8.2045 E-04 \\
3.8804 E-04 \\
1.8333 E-04 \\
8.6565 E-05 \\
4.0867 E-05 \\
1.9292 E-05 \\
9.1074 E-06 \\
1.2957 E-06 \\
2.0301 E-06 \\
9.5861 E-07 \\
4.5267 E-07 \\
2.1378 E-07 \\
1.9134 E-07 \\
1.0000 E+00\end{array}$ & $\begin{array}{l}2.2583 E-02 \\
9.0173 E-02 \\
1.6487 E-01 \\
1.8929 E-01 \\
1.7549 E-01 \\
1.4030 E-01 \\
9.6533 E-02 \\
5.802 B E-02 \\
3.2066 E-02 \\
1.6405 E-02 \\
7.6954 E-03 \\
3.5555 E-03 \\
1.5248 E-03 \\
6.7035 E-04 \\
2.5007 E-04 \\
9.8272 E-05 \\
4.0141 E-05 \\
1.6274 E-05 \\
6.1633 E-06 \\
2.5907 E-06 \\
1.1748 E-06 \\
3.3748 E-07 \\
1.6382 E-07 \\
3.9674 E-08 \\
1.1138 E-0 B \\
1.0000 E-00\end{array}$ & 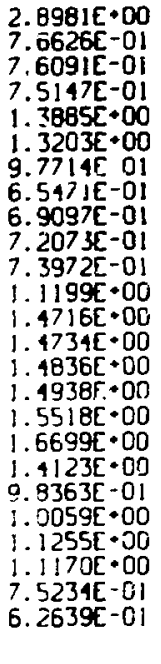 & $\begin{array}{l}6.1629 E-03 \\
2.3709 E-02 \\
4.2468 E-02 \\
1.8206 E-02 \\
1.4325 E-02 \\
3.4360 E-02 \\
2.2671 E-02 \\
1.2943 E-02 \\
6.7547 E-03 \\
3.2696 E-03 \\
1.4715 E-03 \\
6.6028 E-04 \\
2.82 B 2 E-04 \\
1.2360 E-04 \\
1.5582 E-05 \\
1.7940 E-05 \\
7.3577 E-06 \\
3.0158 E-06 \\
1.1722 E-06 \\
5.0293 E-07 \\
2.2076 E-07 \\
6.2409 E-06 \\
3.0526 E-08 \\
7.287 B E-09 \\
8.1627 E-09 \\
2.4748 E-01\end{array}$ & 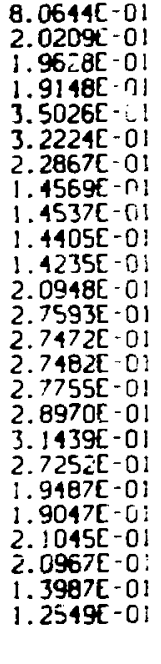 \\
\hline
\end{tabular}


TFat $\theta-x \times$

VERA-IIA 70-GROUP TRANSPORT THEOAY SPECTRA

\begin{tabular}{|c|c|c|c|c|c|c|c|}
\hline Growe & $\begin{array}{r}\text { Enorgy } \\
\text { Boundory } \\
\text { lall } \\
\end{array}$ & $\begin{array}{r}\text { Lothorgy } \\
\text { Hiwellh }\end{array}$ & $\begin{array}{l}\text { Reoctor } \\
\text { Fieseion } \\
\text { Frectivan }\end{array}$ & $\begin{array}{r}\text { Centrol } \\
r l u x \\
\left.\ln / \mathrm{an}^{2} / \mathrm{l}\right)\end{array}$ & $\begin{array}{r}\text { Centrot } \\
\text { Adjoint } \\
\text { Irhololal }\end{array}$ & $\begin{array}{r}\text { Edge } \\
F L \text { ux } \\
\left.\ln / \mathrm{en}^{2} / \mathrm{e}\right) \\
\end{array}$ & $\begin{array}{r}\text { Edge } \\
\text { Ad folnt } \\
\text { Irhotn/n! }\end{array}$ \\
\hline 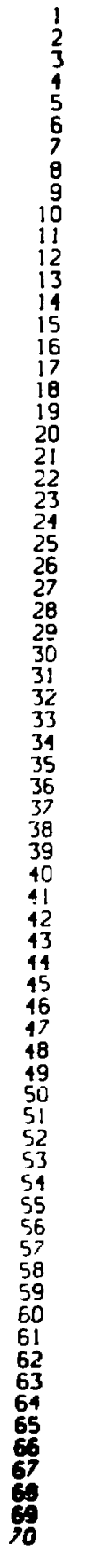 & 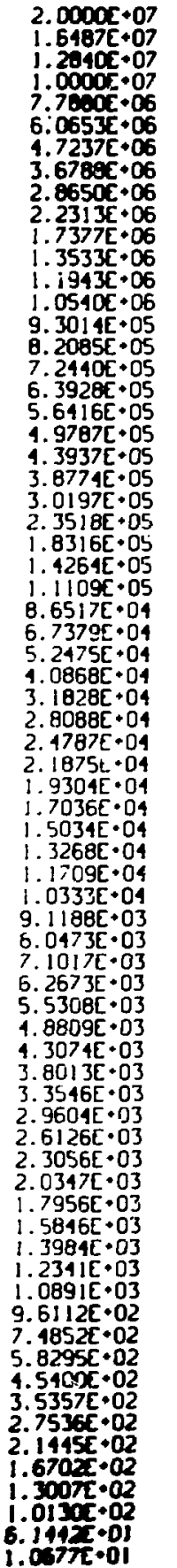 & 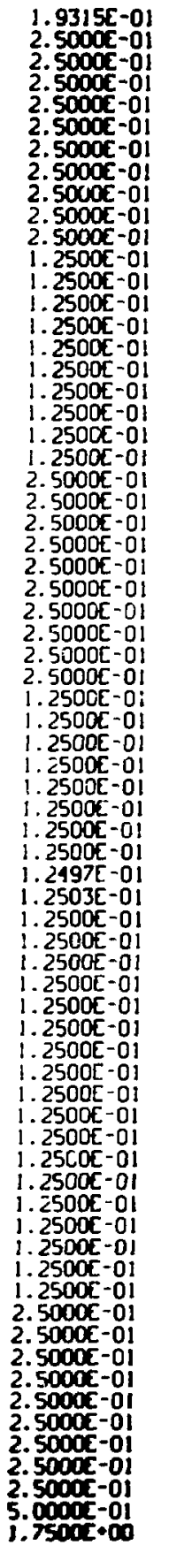 & 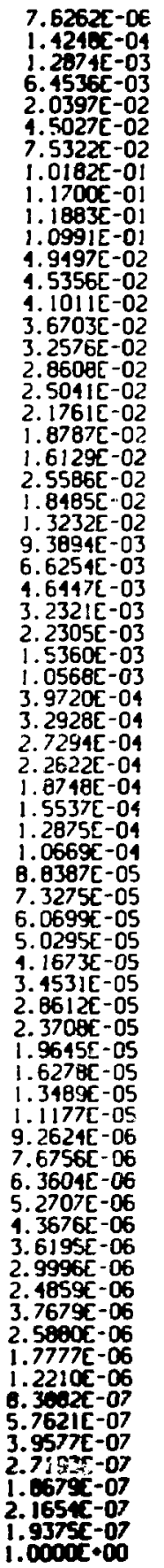 & 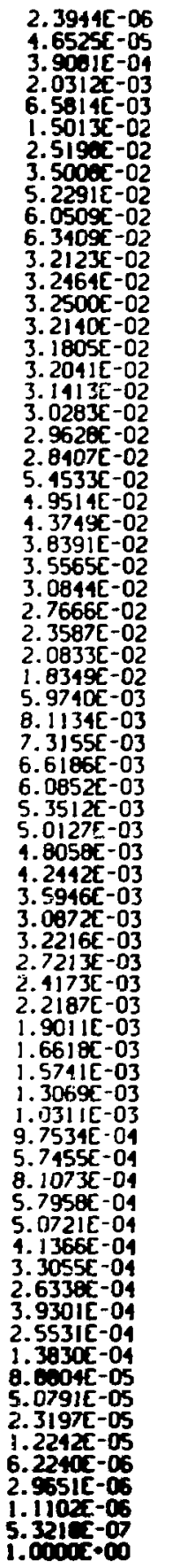 & 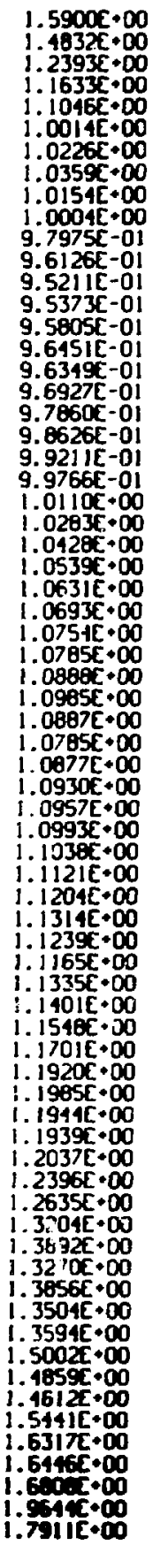 & 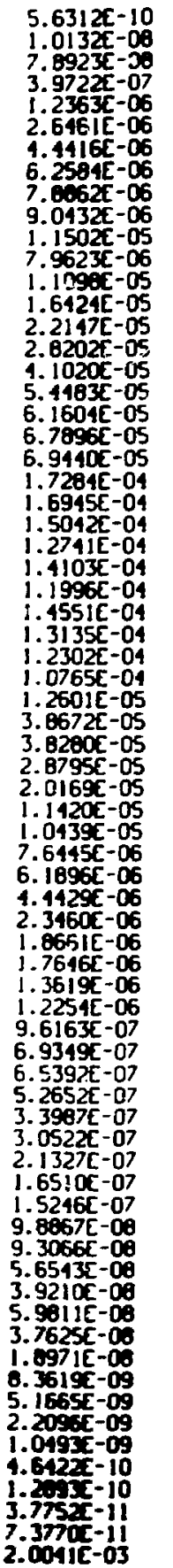 & 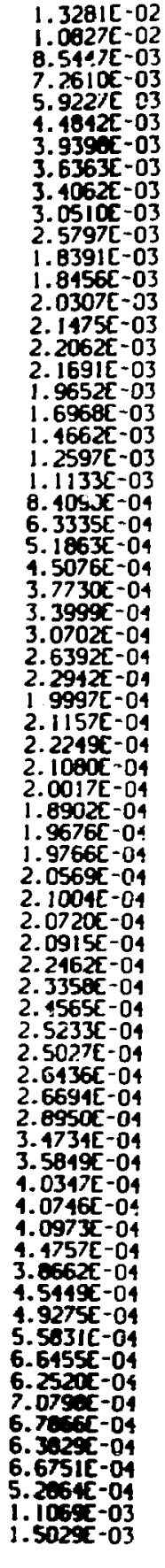 \\
\hline
\end{tabular}


TRBLE $\theta-x \times]$

VEFA-119 70-GROUP ZONE FISSION FRACIIONS

\begin{tabular}{|c|c|c|c|c|}
\hline irsup & $\begin{array}{r}\text { Energy } \\
\text { Boundory } \\
\text { lev } \\
\end{array}$ & $\begin{array}{r}\text { Lethorgy } \\
\text { Wudih }\end{array}$ & Zone 1 & Zene 1 \\
\hline $\begin{array}{l}1 \\
2 \\
3 \\
4 \\
5 \\
6 \\
7 \\
8 \\
9 \\
10 \\
11 \\
12 \\
13 \\
14 \\
15 \\
16 \\
17 \\
18 \\
19 \\
20 \\
21 \\
22 \\
23 \\
24 \\
25 \\
26 \\
4 \\
28 \\
29 \\
30 \\
31 \\
32 \\
33 \\
34 \\
35 \\
36 \\
37 \\
38 \\
39 \\
40 \\
41 \\
12 \\
43 \\
41 \\
45 \\
46 \\
47 \\
48 \\
49 \\
50 \\
51 \\
52 \\
53 \\
54 \\
55 \\
56 \\
65 \\
69 \\
58 \\
59 \\
60 \\
61 \\
70 \\
63 \\
69 \\
69\end{array}$ & 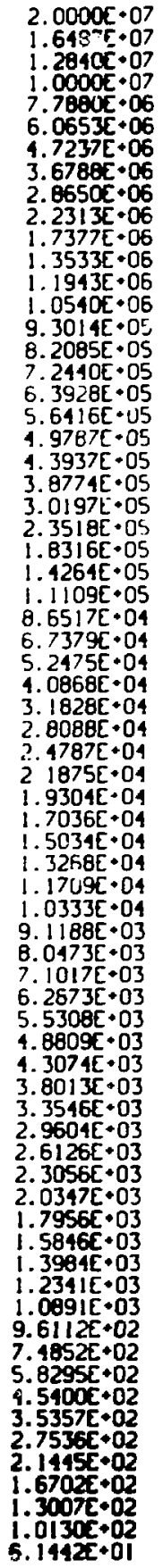 & 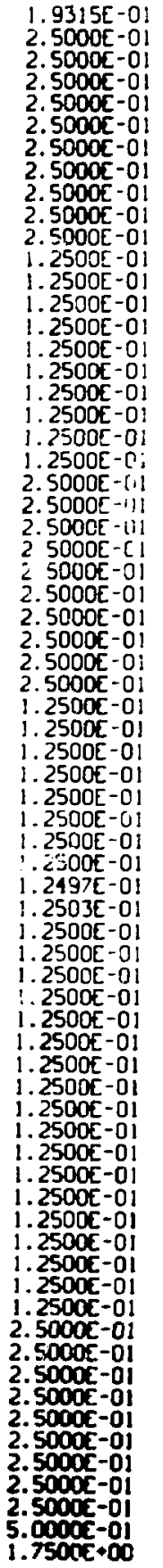 & 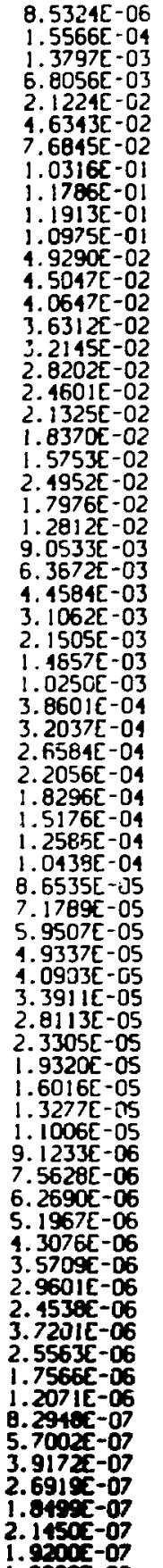 & 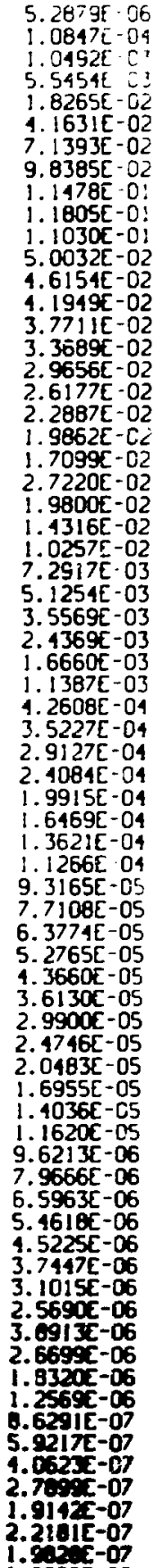 \\
\hline
\end{tabular}


TABLE $8-x \times 1]$

VERA-11A 25-GROUP IRANSPORT THEORY SPECIRA

\begin{tabular}{|c|c|}
\hline cona & $\begin{array}{r}\text { Energy } \\
\text { Boundory } \\
\text { [nv] }\end{array}$ \\
\hline $\begin{array}{l}1 \\
2 \\
3 \\
4 \\
5 \\
6 \\
7 \\
8 \\
9 \\
10 \\
11 \\
12 \\
13 \\
14 \\
15 \\
16 \\
17 \\
18 \\
19 \\
20 \\
21 \\
22 \\
23 \\
24 \\
25\end{array}$ & 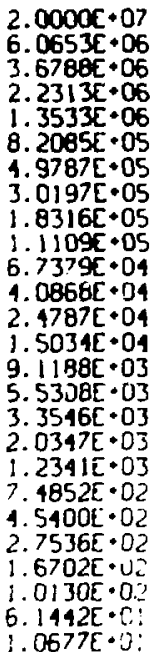 \\
\hline
\end{tabular}

\begin{tabular}{|c|c|}
\hline $\begin{array}{r}\text { Lethorgy } \\
\text { Hydih }\end{array}$ & $\begin{array}{r}\text { Reoctor } \\
\text { Fusscor } \\
\text { Fraction }\end{array}$ \\
\hline 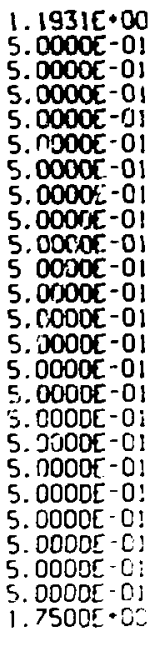 & $\begin{array}{l}2.6289 E-02 \\
1.2035 E-01 \\
2.1882 E-01 \\
2.2673 E-01 \\
1.7257 E-0 ! \\
1.0799 E-01 \\
6.0502 E-02 \\
3.1717 E-02 \\
1.6015 E-02 \\
7.8768 E-03 \\
3.7665 E-03 \\
1.7833 E-03 \\
8.4202 E-04 \\
3.9710 E-04 \\
1.8720 E-04 \\
8.8243 E-05 \\
4.1604 E-05 \\
1.9618 E-05 \\
9.2534 E-06 \\
4.3657 E-06 \\
2.0598 E-06 \\
9.7158 E-07 \\
4.5872 E-07 \\
2.1654 E-07 \\
1.9375 E-07 \\
1.0000 E-0 E\end{array}$ \\
\hline
\end{tabular}

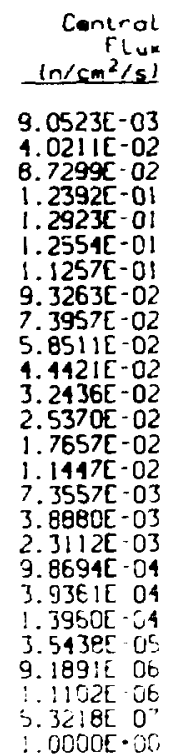

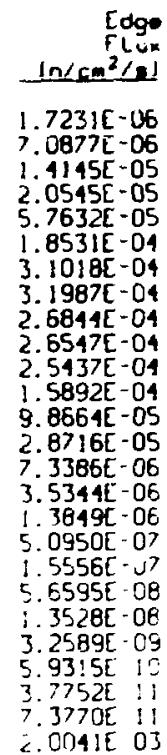

C. ON4IE 03
Edge
Ad foint
Icholnid

Cerit ol Irhotn|s|

2. $4146 E \cdot 00$

7. 4.269 -31

$7.5 .30 \%-01$

$7.2661 \mathrm{E}-01$

$1.1036 E \cdot 00$

$1.4222 \mathrm{E} \cdot 00$

1. $] 920[\cdot 00$

$7.4828 E-01$

$7.6938 \mathrm{E}-01$

$7.8246 \mathrm{E}-0 \mathrm{I}$

7. $9037 \mathrm{E}-0$ !

$1.2021 \mathrm{C}+00$

$1.5980 C \cdot 00$

$1.6276[\cdot 00$

1. $65325+00$

i. $70995 \cdot 00$

$1.7578[+00$

1. $9128 \mathrm{5} * 00$

1. $49095 \cdot 00$

$1.04935 \cdot 00$

$1.08140 \cdot 00$

$1.16535 \cdot 0$ ?

$\therefore 22025 \cdot 00$

$.2081: 0$ :

TABLE $8-x \times] / 1$

VEAA-11A 25 -GROUP ZONE FISSION FRACTIONS

\begin{tabular}{|c|c|c|c|c|}
\hline م- & $\begin{array}{r}\text { Enorgy } \\
\text { Boundory } \\
\text { lev }\end{array}$ & $\begin{array}{r}\text { Lethorgy } \\
\text { Hudif }\end{array}$ & Sore & Reflector \\
\hline $\begin{array}{l}1 \\
2 \\
3 \\
1 \\
5 \\
6 \\
7 \\
8 \\
9 \\
10 \\
11 \\
12 \\
13 \\
14 \\
15 \\
16 \\
17 \\
18 \\
19 \\
20 \\
21 \\
22 \\
23 \\
24 \\
25\end{array}$ & 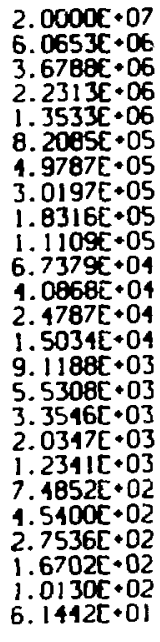 & $\begin{array}{l}\text { 1. } 1931 E-00 \\
5.0000 E-01 \\
5.0000 E-01 \\
5.0000 E-01 \\
5.0000 E-01 \\
5.0000 E-01 \\
5.0000 E-01 \\
5.0000 E-01 \\
5.0000 E-01 \\
5.0000 E-01 \\
5.0000 E-01 \\
5.0000 E-01 \\
5.0000 E-01 \\
5.0000 E-01 \\
5.0000 E-01 \\
5.0000 E-01 \\
5.0000 E-01 \\
5.0000 E-01 \\
5.0000 E-01 \\
5.0000 E-01 \\
5.0000 E-01 \\
5.0000 E-01 \\
5.0000 E-01 \\
5.0000 E-01 \\
1.7500 E-00\end{array}$ & $\begin{array}{l}2.9573[-02 \\
1.2319[-01 \\
2.2101[-01 \\
2.2888[-01 \\
1.7130[-01 \\
1.0627[-01 \\
5.9076[-02 \\
3.0788[-02 \\
1.5421[-02 \\
7.5646[-03 \\
3.6362[-03 \\
1.7314[-03 \\
6.2111[-04 \\
3.8857[-04 \\
1.8366[-04 \\
8.6754[-05 \\
1.0968[-05 \\
1.9344[-05 \\
9.1339[-06 \\
1.3129[-06 \\
2.0365[-06 \\
9.6174[-07 \\
1.5418[-07 \\
2.1450[-07 \\
1.9200[-07 \\
1.0000[+00\end{array}$ & $\begin{array}{l}2.4973 E-02 \\
1.1302 E-0 ! \\
2.1317 E-01 \\
2.2835 E-01 \\
1.7585 E-01 \\
1.1241 E-01 \\
6.4182 E-02 \\
3.4116 E-02 \\
1.7548 E-02 \\
8.6823 E-03 \\
1.1028 E-03 \\
1.9171 E-03 \\
8.9595 E-01 \\
1.1914 E-04 \\
1.9633 E-04 \\
9.2085 E-05 \\
4.3245 E-05 \\
2.0325 E-05 \\
9.5617 E-06 \\
4.5020 E-06 \\
2.1198 E-06 \\
9.9839 E-07 \\
4.7041 E-07 \\
2.2181 E-07 \\
1.9828 E-07 \\
1.0000 E-00\end{array}$ \\
\hline
\end{tabular}


Thate $\theta-x \times 1 y$

ZPR-3-48 70-GROUP IRAWSPORT IMEORY SPECTRA

\begin{tabular}{|c|c|c|c|c|c|c|c|}
\hline $\cos 9$ & $\begin{array}{r}\text { Energy } \\
\text { Boundory } \\
(\ln y)\end{array}$ & $\begin{array}{r}\text { Lethorgy } \\
\text { Hidll }\end{array}$ & $\begin{array}{r}\text { Reoclor } \\
\text { Fievion } \\
\text { frachions }\end{array}$ & $\begin{array}{r}\text { Centrol } \\
F l u x \\
\ln \left\langle\cos ^{2} / \mathrm{l}\right)\end{array}$ & $\begin{array}{r}\text { Central } \\
\text { Ad joint } \\
\text { irtop/n/nl }\end{array}$ & $\begin{array}{r}E \text { dg } \\
F \operatorname{lux} \\
\ln \left(a n^{2}(n)\right.\end{array}$ & $\begin{array}{r}\text { Edge } \\
\text { Ard jolnt } \\
\text { Irhololel }\end{array}$ \\
\hline $\begin{array}{l}1 \\
2 \\
3 \\
4 \\
5 \\
6 \\
7 \\
6 \\
9 \\
10 \\
11 \\
12 \\
13 \\
14 \\
15 \\
16 \\
17 \\
18 \\
19 \\
20 \\
21 \\
22 \\
23 \\
24 \\
25 \\
26 \\
27 \\
28 \\
29 \\
30 \\
31 \\
32 \\
33 \\
34 \\
35 \\
36 \\
37 \\
38 \\
39 \\
40 \\
41 \\
42 \\
13 \\
44 \\
45 \\
46 \\
17 \\
18 \\
49 \\
50 \\
51 \\
52 \\
53 \\
54 \\
55 \\
56 \\
57 \\
58 \\
59 \\
60 \\
61 \\
62 \\
63 \\
64 \\
65 \\
66 \\
67 \\
60 \\
69 \\
70\end{array}$ & 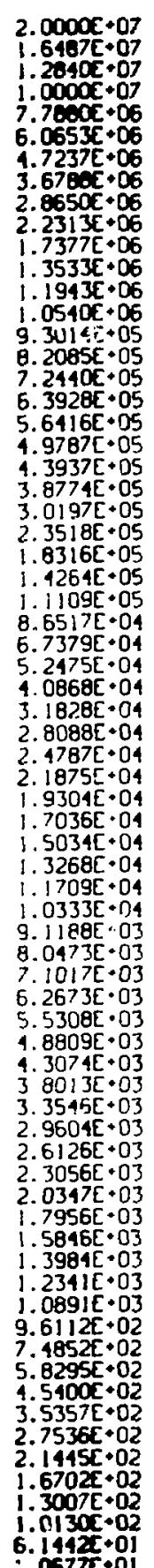 & $\begin{array}{l}1.9315 E-01 \\
2.5000 E-01 \\
2.5000 E-01 \\
2.5000 E-01 \\
2.5000 E-01 \\
2.5000 E-01 \\
2.5000 E-01 \\
2.5000 E-01 \\
2.5000 E-01 \\
2.5000 E-01 \\
2.5000 E-01 \\
1.2500 E-01 \\
1.2500 E-01 \\
1.2500 E-01 \\
1.2500 E-01 \\
1.2500 E-01 \\
1.2500 E-01 \\
1.2500 E-01 \\
1.2500 E-01 \\
1.2500 E-01 \\
1.2500 E-01 \\
2.5000 E-01 \\
2.5000 E-01 \\
2.5000 E-01 \\
2.5000 E-01 \\
2.5000 E-01 \\
2.5000 E-01 \\
2.5000 E-01 \\
2.5000 E-01 \\
2.5000 E-01 \\
2.5000 E-01 \\
1.2500 E-01 \\
1.2500 E-01 \\
1.2500 E-01 \\
1.2500 E-01 \\
1.2500 E-01 \\
1.2500 E-01 \\
1.2500 E-01 \\
1.2500 E-01 \\
1.2497 E-01 \\
1.2503 E-01 \\
1.2500 E-01 \\
1.2500 E-01 \\
1.2500 E-01 \\
1.2500 E-01 \\
1.2500 E-01 \\
1.2500 E-01 \\
1.2500 E-01 \\
1.2500 E-01 \\
1.2500 E-01 \\
1.2500 E-01 \\
1.2500 E-01 \\
1.2500 E-01 \\
1.2500 E-01 \\
1.2500 E-01 \\
1.2500 E-01 \\
1.2500 E-01 \\
1.2500 E-01 \\
1.2500 E-01 \\
2.5000 E-01 \\
2.5000 E-01 \\
2.5000 E-01 \\
2.5000 E-01 \\
2.5000 E-01 \\
2.5000 E-01 \\
2.5000 E-01 \\
2.5000 E-01 \\
2.5000 E-01 \\
5.0000 E-01 \\
1.501\end{array}$ & 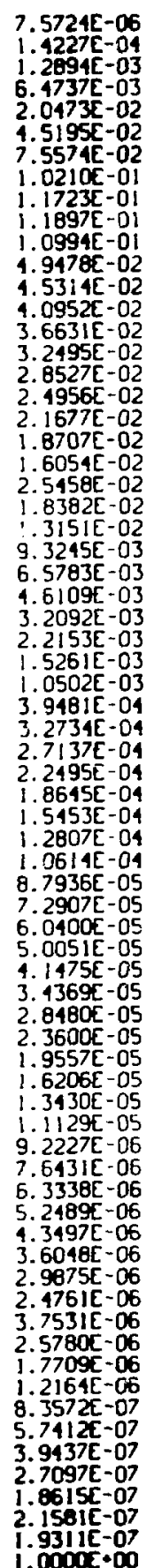 & 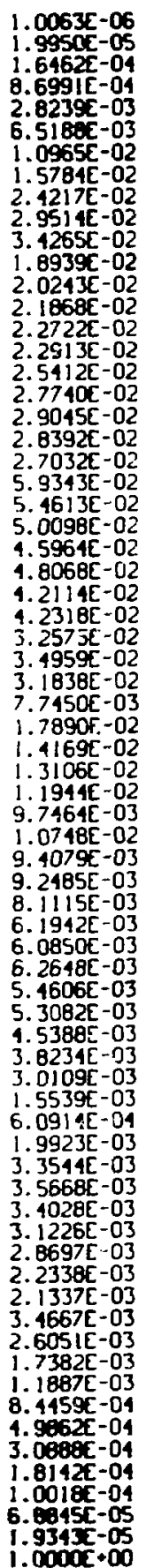 & $\begin{array}{l}1.9290 E+00 \\
1.7367 E+00 \\
1.4607 E+00 \\
1.3711 E+00 \\
1.2558 E+00 \\
1.1094 E+00 \\
1.0991 E+00 \\
1.0950 E+00 \\
1.0738 E+00 \\
1.0351 E+00 \\
9.7686 E-01 \\
9.1885 E-01 \\
9.0568 E-01 \\
9.0172 E-01 \\
9.0223 E-01 \\
9.0353 E-01 \\
9.0008 E-01 \\
8.9690 E-01 \\
8.9721 E-01 \\
8.9701 E-01 \\
8.9526 E-01 \\
8.8918 E-01 \\
8.8426 E-01 \\
8.8004 E-01 \\
8.7151 E-01 \\
8.6343 E-01 \\
8.5449 E-01 \\
8.4125 E-01 \\
8.3571 E-01 \\
8.2850 E-01 \\
8.2560 E-01 \\
8.2655 E-01 \\
8.2357 E-01 \\
8.2467 E-01 \\
8.3086 E-01 \\
8.3496 E-01 \\
8.4001 E-01 \\
8.4259 E-01 \\
8.5011 E-01 \\
8.5598 E-01 \\
8.6415 E-01 \\
8.7457 E-01 \\
8.7468 E-01 \\
8.7815 E-01 \\
8.8987 E-01 \\
8.9848 E-01 \\
9.1010 E-01 \\
9.1911 E-01 \\
9.2850 E-01 \\
9.3289 E-01 \\
9.3638 E-01 \\
9.3752 E-01 \\
9.4449 E-01 \\
9.6795 E-01 \\
9.7875 E-01 \\
1.0196 E+00 \\
1.0576 E+00 \\
1.0065 E+00 \\
1.0878 E+00 \\
1.0648 E+00 \\
1.0995 E+00 \\
1.2161 E+00 \\
1.1478 E+00 \\
1.1587 E+00 \\
1.2351 E+00 \\
1.2062 E+00 \\
1.2751 E+00 \\
1.3431 E+00 \\
1.6309 E+00 \\
1.5636 E+00\end{array}$ & 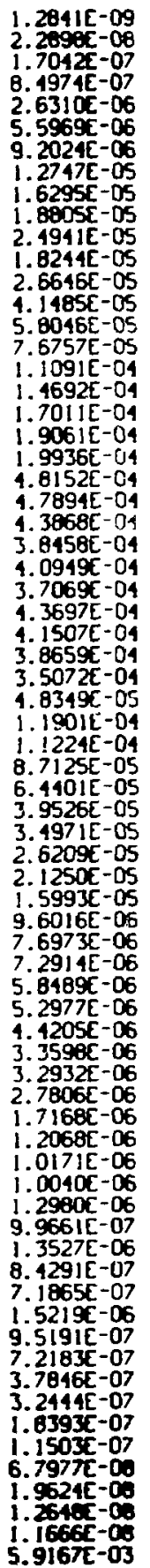 & 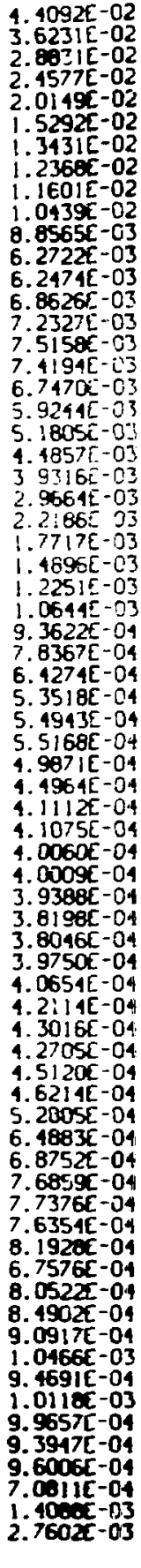 \\
\hline
\end{tabular}


TFaLe $B-X X Y$

2PR-3-48 70-GROUP ZONE FISSION FRACIIONS

\begin{tabular}{|c|c|c|c|c|}
\hline Cor & $\begin{array}{r}\text { Energy } \\
\text { Boundory } \\
\text { lnvII }\end{array}$ & $\begin{array}{l}\text { Lethorgy } \\
\text { Midlf }\end{array}$ & $\mathrm{Co}$ & Letor \\
\hline $\begin{array}{c}1 \\
2 \\
3 \\
1 \\
5 \\
6 \\
7 \\
6 \\
9 \\
10 \\
11 \\
12 \\
13 \\
14 \\
15 \\
16 \\
17 \\
18 \\
19 \\
20 \\
21 \\
22 \\
23 \\
24 \\
25 \\
26 \\
27 \\
28 \\
29 \\
30 \\
31 \\
32 \\
33 \\
34 \\
35 \\
36 \\
37 \\
38 \\
39 \\
67 \\
40 \\
65 \\
61 \\
63 \\
42 \\
43 \\
44 \\
45 \\
59 \\
46 \\
47 \\
48 \\
49 \\
50 \\
51 \\
52 \\
53 \\
54 \\
55 \\
56 \\
57 \\
65\end{array}$ & 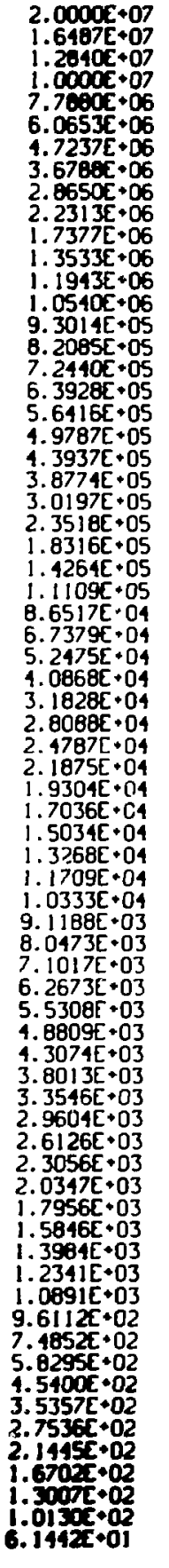 & 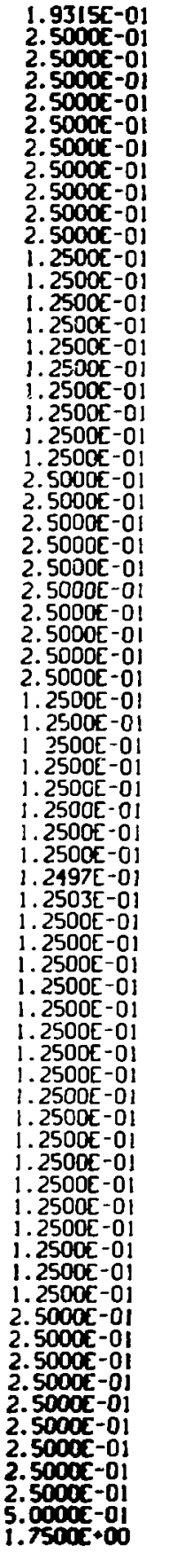 & $\begin{array}{l}7.8695 E-06 \\
1.1662 E-04 \\
1.3190 E-03 \\
6.5992 E-03 \\
2.0742 E-02 \\
4.5619 E-02 \\
7.6061 E-02 \\
1.0253 E-01 \\
1.1751 E-01 \\
1.1907 E-01 \\
1.0990 E-01 \\
4.9416 E-02 \\
1.5218 E-02 \\
4.0838 E-02 \\
3.6509 E-02 \\
3.2359 E-02 \\
2.8399 E-02 \\
2.4814 E-02 \\
2.1535 E-02 \\
1.8570 E-02 \\
1.5932 E-02 \\
2.5251 E-02 \\
1.8215 E-02 \\
1.3011 E-02 \\
9.2127 E-03 \\
6.1915 E-03 \\
1.5485 E-03 \\
3.1671 E-03 \\
2.1889 E-03 \\
1.5096 E-03 \\
1.0399 E-03 \\
3.9123 E-04 \\
3.2450 E-04 \\
2.6912 E-04 \\
2.2317 E-04 \\
1.8504 E-04 \\
1.5341 E-04 \\
1.2718 E-04 \\
1.0543 E-04 \\
8.7375 E-05 \\
7.2461 E-05 \\
6.0044 E-05 \\
4.9768 E-05 \\
1.1249 E-05 \\
3.4188 E-05 \\
2.8335 E-05 \\
2.3484 E-05 \\
1.9464 E-05 \\
1.6132 E-05 \\
1.3370 E-05 \\
1.1081 E-05 \\
9.1844 E-06 \\
7.6122 E-06 \\
6.3090 E-06 \\
5.2290 E-06 \\
4.3338 E-06 \\
3.5921 E-06 \\
2.9773 E-06 \\
2.4677 E-06 \\
3.7408 E-06 \\
2.5700 E-06 \\
1.7657 E-06 \\
1.2131 E-06 \\
8.3351 E-07 \\
5.7271 E-07 \\
3.9349 E-07 \\
2.7039 E-07 \\
1.8579 E-07 \\
2.1540 E-07 \\
1.9277 E-07 \\
\end{array}$ & 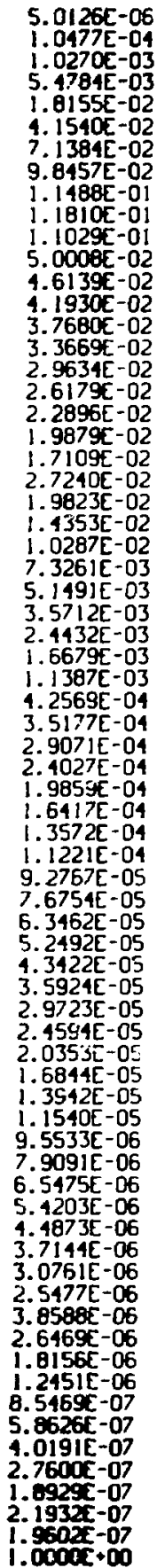 \\
\hline
\end{tabular}


TFBLE $B-x \times Y 1$

ZPR-3-48 25-GROUP TRANSPQRT THEORY SPECTRA

\begin{tabular}{|c|c|c|c|c|c|c|c|}
\hline cone & $\begin{array}{r}\text { Energy } \\
\text { Boundory } \\
\text { InYII }\end{array}$ & $\begin{array}{r}\text { Lethorgy } \\
\text { Hideh }\end{array}$ & $\begin{array}{r}\text { Reoctor } \\
\text { Fiovion } \\
\text { Frections }\end{array}$ & $\begin{array}{r}\text { Central } \\
\text { flux } \\
\left.\ln / \operatorname{cm}^{2} / \mathrm{s}\right) \\
\end{array}$ & $\begin{array}{r}\text { Central } \\
\text { Ad jount } \\
\text { Irhotn/el }\end{array}$ & $\begin{array}{r}\text { Edge } \\
\text { Flux } \\
\ln \left\langle\cos ^{2} / u l\right. \\
\end{array}$ & $\begin{array}{r}\text { Edge } \\
\text { Pdd joint } \\
\text { Irhoth/el }\end{array}$ \\
\hline $\begin{array}{r}1 \\
2 \\
3 \\
4 \\
5 \\
6 \\
7 \\
8 \\
9 \\
10 \\
11 \\
12 \\
13 \\
14 \\
15 \\
16 \\
17 \\
18 \\
19 \\
20 \\
21 \\
22 \\
23 \\
24 \\
25\end{array}$ & $\begin{array}{l}2.0000 E+07 \\
6.0553 E+06 \\
3.6786 E+06 \\
2.2313 E+06 \\
1.3533 E+06 \\
8.2085 E+05 \\
4.9787 E+05 \\
3.0197 E+05 \\
1.8316 E+05 \\
1.1109 E+05 \\
6.7379 E+04 \\
4.0868 E+04 \\
2.4787 E+04 \\
1.5034 E+04 \\
9.1188 E+03 \\
5.5308 E+03 \\
3.3546 E+03 \\
2.0347 E+03 \\
1.2341 E+03 \\
7.4852 E+02 \\
4.5400 E+02 \\
2.7536 E+02 \\
1.6702 E+02 \\
1.0130 E+02 \\
6.1442 E+01\end{array}$ & $\begin{array}{l}\text { 1. } 1931 E-00 \\
5.0000 E-01 \\
5.0000 E-01 \\
5.0000 E-01 \\
5.0000 E-01 \\
5.0000 E-01 \\
5.0000 E-01 \\
5.0000 E-01 \\
5.0000 E-01 \\
5.0000 E-01 \\
5.0000 E-01 \\
5.0000 E-01 \\
5.0000 E-01 \\
5.0000 E-01 \\
5.0000 E-01 \\
5.0000 E-01 \\
5.0000 E-01 \\
5.0000 E-01 \\
5.0000 E-01 \\
5.0000 E-01 \\
5.0000 E-01 \\
5.0000 E-01 \\
5.0000 E-01 \\
5.0000 E-01 \\
1.7500 E+00\end{array}$ & $\begin{array}{l}2.8386 E-02 \\
1.2077 E-01 \\
2.1934 E-01 \\
2.2891 E-01 \\
1.7237 E-01 \\
1.0765 E-01 \\
6.0219 E-02 \\
3.1533 E-02 \\
1.5903 E-02 \\
7.8201 E-03 \\
3.7414 E-03 \\
1.7723 E-03 \\
8.3730 E-04 \\
3.9505 E-04 \\
1.8629 E-04 \\
8.7842 E-05 \\
1.1424 E-05 \\
1.9537 E-05 \\
9.2166 E-06 \\
4.3499 E-06 \\
2.0521 E-06 \\
9.6848 E-07 \\
1.5712 E-07 \\
2.1581 E-07 \\
1.9311 \mathrm{~L}-07 \\
1.0000 E+00\end{array}$ & $\begin{array}{l}3.8794 E-03 \\
1.7484 E-02 \\
4.0001 E-02 \\
6.3778 E-02 \\
8.3772 E-02 \\
1.0511 E-01 \\
1.1477 E-01 \\
1.0471 E-01 \\
9.4032 E-02 \\
8.4432 E-02 \\
6.7534 E-02 \\
5.7473 E-02 \\
1.8965 E-02 \\
3.7516 E-02 \\
2.4005 E-02 \\
1.6681 E-02 \\
7.5096 E-03 \\
1.2962 E-02 \\
7.8341 E-03 \\
4.3433 E-03 \\
2.0333 E-03 \\
8.0749 E-04 \\
2.8159 E-04 \\
6.8845 E-05 \\
1.9343 E-05 \\
1.0500 E-00\end{array}$ & $\begin{array}{l}2.8602 E+00 \\
8.1468 E-01 \\
8.0007 E-01 \\
7.4221 E-01 \\
1.3385 E+00 \\
1.3272 E+00 \\
9.8917 E-01 \\
5.5084 E-01 \\
6.4001 E-01 \\
6.2665 E-01 \\
6.1392 E-01 \\
9.1328 E-01 \\
1.2286 E+00 \\
1.2590 E+00 \\
1.2975 E+00 \\
1.3487 E+00 \\
1.3838 E+00 \\
1.4844 E+00 \\
1.1654 E+00 \\
8.5424 E-01 \\
8.5083 E-01 \\
9.3009 E-01 \\
9.6585 E-01 \\
6.0164 E-01 \\
5.7679 E-01\end{array}$ & $\begin{array}{l}3.6753 E-06 \\
1.4799 E-05 \\
2.9042 E-05 \\
4.3746 E-05 \\
1.4442 E-04 \\
5.0469 E-04 \\
8.7148 E-04 \\
9.1762 E-04 \\
7.9408 E-04 \\
8.0766 E-04 \\
8.0166 E-04 \\
5.1807 E-04 \\
3.0329 E-04 \\
9.8423 E-05 \\
3.0439 E-05 \\
1.6371 E-05 \\
6.7213 E-06 \\
4.6514 E-06 \\
3.0834 E-06 \\
1.6737 E-06 \\
7.0290 E-07 \\
2.9895 E-07 \\
8.7602 E-08 \\
1.2648 E-08 \\
1.1566 E-08 \\
5.9167 E-03\end{array}$ & $\begin{array}{l}5.6766 E-02 \\
1.0596 E-02 \\
8.84 E I E-03 \\
7.1160 E-03 \\
9.8181 E-03 \\
1.0184 E-02 \\
5.0162 E-03 \\
1.9127 E-03 \\
1.2030 E-03 \\
\text { E. } 4456 E-04 \\
6.3445 E-04 \\
6.3721 E-04 \\
7.0501 E-04 \\
5.9219 E-04 \\
5.7787 E-04 \\
6.3802 E-04 \\
8.5824 E-04 \\
1.1529 E-03 \\
8.5952 E-04 \\
7.2148 E-04 \\
7.2257 E-04 \\
7.1419 E-04 \\
6.1538 E-04 \\
5.1970 E-04 \\
1.0182 E-03\end{array}$ \\
\hline
\end{tabular}

TABLE $B-x \times$ VII

ZPR-3-48 25-GROUP ZONE F:SSION FRACTIONS

\begin{tabular}{|c|c|c|c|c|}
\hline$w_{2}$ & $\begin{array}{r}\text { Enorgy } \\
\text { Boundory } \\
(\mathrm{e} Y) \\
\end{array}$ & $\begin{array}{r}\text { Lethorgy } \\
\text { Whdth }\end{array}$ & Core & Reflecter \\
\hline $\begin{array}{l}1 \\
2 \\
3 \\
1 \\
5 \\
6 \\
7 \\
6 \\
9 \\
10 \\
11 \\
12 \\
13 \\
14 \\
15 \\
16 \\
17 \\
18 \\
19 \\
20 \\
21 \\
22 \\
23 \\
24 \\
25\end{array}$ & $\begin{array}{l}2.0000 E+07 \\
6.0653 E+06 \\
3.6788 E+06 \\
2.2313 E+06 \\
1.3533 E+06 \\
8.2085 E+05 \\
4.9787 E+05 \\
3.0197 E+05 \\
1.8316 E+05 \\
1.1109 E+05 \\
6.7379 E+04 \\
4.0868 E+04 \\
2.4787 E+04 \\
1.5034 E+01 \\
9.1188 E+03 \\
5.5308 E+03 \\
3.3546 E+03 \\
2.0347 E+03 \\
1.2341 E+03 \\
7.4852 E+02 \\
4.5400 E+02 \\
2.7536 E+02 \\
1.6702 E+02 \\
1.0130 E+02 \\
6.1442 E+01\end{array}$ & $\begin{array}{l}\text { 1. } 1931 E+00 \\
5.0000 E-01 \\
5.0000 E-01 \\
5.0000 E-01 \\
5.0000 E-01 \\
5.0000 E-01 \\
5.0000 E-01 \\
5.0000 E-01 \\
5.0000 E-01 \\
5.0000 E-01 \\
5.0000 E-01 \\
5.0000 E-01 \\
5.0000 E-01 \\
5.0000 E-01 \\
5.0000 E-01 \\
5.0000 E-01 \\
5.0000 E-01 \\
5.0000 E-01 \\
5.0000 E-01 \\
5.0000 E-01 \\
5.0000 E-01 \\
5.0000 E-01 \\
5.0000 E-01 \\
5.0000 E-01 \\
1.7500 E-00\end{array}$ & $\begin{array}{l}2.8806 E-02 \\
1.2168 E-01 \\
2.2003 E-01 \\
2.2697 E-01 \\
1.7198 E-01 \\
1.0711 E-01 \\
5.9753 E-02 \\
3.1226 E-02 \\
1.5704 E-02 \\
7.7156 E-03 \\
3.6965 E-03 \\
1.7557 E-03 \\
8.3074 E-04 \\
3.9245 E-04 \\
1.8525 E-04 \\
8.7416 E-05 \\
4.1248 E-05 \\
1.9464 E-05 \\
9.1858 E-06 \\
1.3357 E-06 \\
2.0466 E-06 \\
9.6620 E-07 \\
4.5618 E-07 \\
2.1540 E-07 \\
1.9277 E-07 \\
1.0000 E-00\end{array}$ & $\begin{array}{l}2.4770 E-02 \\
1.1292 E-01 \\
2.1333 E-01 \\
2.2840 E-01 \\
1.7575 E-01 \\
1.1238 E-01 \\
6.4228 E-02 \\
3.4176 E-02 \\
1.7613 E-02 \\
8.7203 E-03 \\
4.1111 E-03 \\
1.9161 E-03 \\
8.9374 E-04 \\
4.1745 E-04 \\
1.9530 E-04 \\
9.1514 E-05 \\
4.2944 E-05 \\
2.0170 E-05 \\
9.4825 E-06 \\
4.4626 E-06 \\
2.0997 E-06 \\
9.8817 E-07 \\
4.6529 E-07 \\
2.1932 E-07 \\
1.9602 E-07 \\
1.0000 E+00\end{array}$ \\
\hline
\end{tabular}


TFBLE $B-x \times V] \|$

2EGRA-3 70-GROUP TRAWSPORT THEORY SPECTRA

\begin{tabular}{|c|c|c|c|c|c|c|c|}
\hline oun & $\begin{array}{r}\text { Enargy } \\
\text { Boundory } \\
\text { levI }\end{array}$ & $\begin{array}{r}\text { Lethorgy } \\
\text { Hidth }\end{array}$ & $\begin{array}{r}\text { Reoctor } \\
\text { Fiseion } \\
\text { Iroctionn }\end{array}$ & 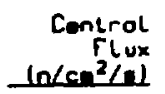 & $\begin{array}{r}\text { Centrol } \\
\text { Rd joint } \\
\text { Irholn } / \mathrm{l} \text {. }\end{array}$ & $\begin{array}{r}E d \\
\ln / a^{2}\end{array}$ & $\begin{array}{r}\text { Edge } \\
\text { Ad joint } \\
\text { Irholn/nl }\end{array}$ \\
\hline $\begin{array}{l}1 \\
2 \\
3 \\
1 \\
5 \\
6 \\
7 \\
6 \\
9 \\
10 \\
11 \\
12 \\
13 \\
14 \\
15 \\
16 \\
17 \\
18 \\
19 \\
20 \\
21 \\
22 \\
23 \\
24 \\
25 \\
26 \\
27 \\
28 \\
29 \\
30 \\
31 \\
32 \\
33 \\
31 \\
35 \\
36 \\
37 \\
38 \\
39 \\
40 \\
41 \\
42 \\
43 \\
44 \\
45 \\
46 \\
47 \\
48 \\
49 \\
50 \\
51 \\
52 \\
53 \\
54 \\
55 \\
56 \\
57 \\
58 \\
59 \\
60 \\
61 \\
62 \\
63 \\
64 \\
65 \\
66 \\
67 \\
68 \\
69 \\
70\end{array}$ & $\begin{array}{l}2.0000 E+07 \\
1.6487 E+07 \\
1.2640 E+07 \\
1.0000 E+07 \\
7.7600 E+06 \\
6.0653 E+06 \\
1.7237 E+05 \\
3.6788 E+06 \\
2.8650 E+06 \\
2.2313 E+06 \\
1.7377 E+06 \\
1.3533 E+06 \\
1.1943 E+06 \\
1.0540 E+06 \\
9.3014 E+05 \\
8.2085 E+05 \\
7.2440 E+05 \\
6.3928 E+05 \\
5.6416 E+05 \\
4.9787 E+05 \\
1.3937 E+05 \\
3.8774 E+05 \\
3.0197 E+05 \\
2.3518 E+05 \\
1.8316 E+05 \\
1.4264 E+05 \\
1.1109 E+05 \\
8.6517 E+04 \\
6.7379 E+04 \\
5.2475 E+04 \\
4.0868 E+04 \\
3.1828 E+04 \\
2.8088 E+04 \\
2.4787 E+04 \\
2.1875 E+04 \\
1.9304 E+04 \\
1.7036 E+04 \\
1.5034 E+04 \\
1.3268 E+04 \\
1.1709 E+04 \\
1.0333 E+04 \\
9.1188 E+03 \\
8.0473 E+03 \\
7.1017 E+03 \\
6.2673 E+03 \\
5.5308 E+03 \\
4.8809 E+03 \\
4.3074 E+03 \\
3.8013 E+03 \\
3.3546 E+03 \\
2.9604 E+03 \\
2.6126 E+03 \\
2.3056 E+03 \\
2.0347 E+03 \\
1.7956 E+03 \\
1.5846 E+03 \\
1.3984 E+03 \\
1.2341 E+03 \\
1.0891 E+03 \\
9.6112 E+02 \\
7.4652 E+02 \\
5.8295 E+02 \\
4.5400 E+02 \\
3.5357 E+02 \\
2.7536 E+02 \\
2.1445 E+02 \\
1.6702 E+02 \\
1.3007 E+02 \\
1.0130 E+02\end{array}$ & 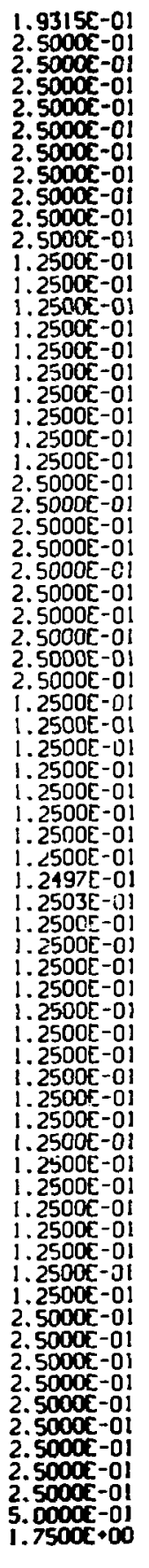 & $\begin{array}{l}7.1537 E-06 \\
1.3627 E-04 \\
1.2471 E-03 \\
6.3099 E-03 \\
2.0079 E-02 \\
1.4549 E-02 \\
7.1800 E-02 \\
1.0139 E-01 \\
1.1675 E-01 \\
1.1876 E-01 \\
1.0999 E-01 \\
4.9577 E-02 \\
4.5469 E-02 \\
4.1139 E-02 \\
3.6836 E-02 \\
3.2723 E-02 \\
2.6743 E-02 \\
2.5189 E-02 \\
2.1907 E-02 \\
1.8926 E-02 \\
1.6253 E-02 \\
2.5793 E-02 \\
1.8651 E-02 \\
1.3370 E-02 \\
9.4997 E-03 \\
6.7113 E-03 \\
1.7065 E-03 \\
3.2736 E-03 \\
2.2563 E-03 \\
1.5519 E-03 \\
1.0665 E-03 \\
1.0058 E-04 \\
3.3193 E-04 \\
2.7503 E-04 \\
2.2787 E-04 \\
1.8678 E-04 \\
1.5639 E-04 \\
1.2956 E-04 \\
1.0733 E-04 \\
8.8809 E-05 \\
7.3672 E-05 \\
6.1013 E-05 \\
5.0543 E-05 \\
4.1870 E-05 \\
3.4687 E-05 \\
2.8736 E-05 \\
2.3806 E-05 \\
1.9723 E-05 \\
1.6340 E-05 \\
1.3538 E-05 \\
1.1217 E-05 \\
9.2940 E-06 \\
7.7008 E-06 \\
6.3806 E-06 \\
5.2868 E-06 \\
1.3605 E-06 \\
3.6297 E-06 \\
3.0078 E-06 \\
2.4926 E-06 \\
3.7775 E-06 \\
2.5942 E-06 \\
1.7817 E-06 \\
1.2236 E-06 \\
8.1052 E-07 \\
5.7731 E-07 \\
3.9648 E-07 \\
2.7240 E-07 \\
1.8709 E-07 \\
2.1697 E-07 \\
1.9402 E-07 \\
1.0030 E+00\end{array}$ & 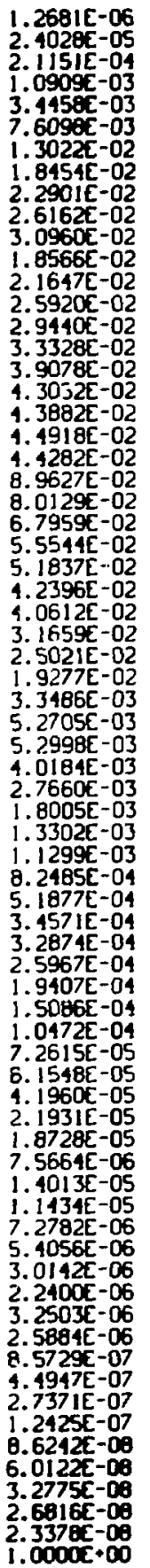 & 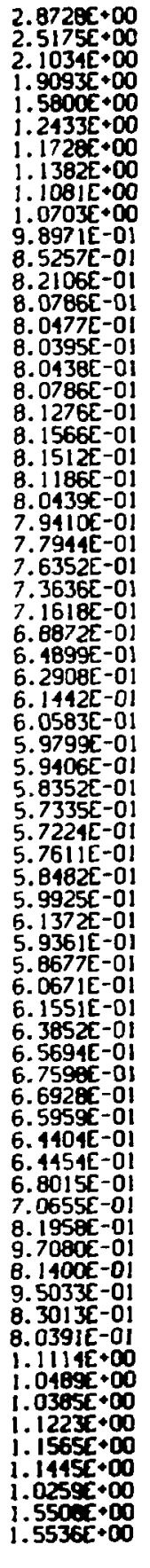 & 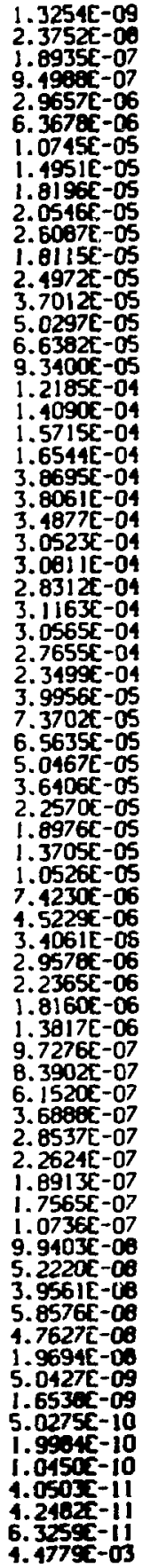 & 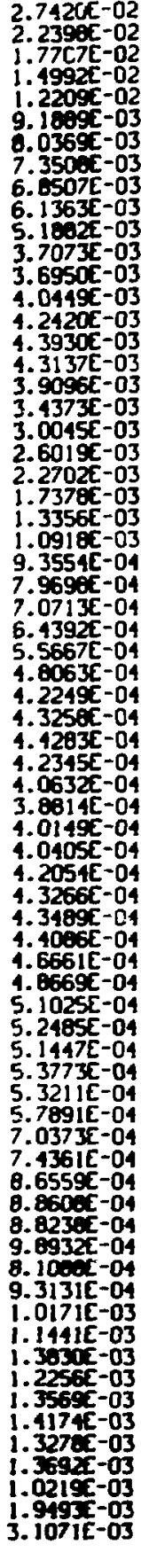 \\
\hline
\end{tabular}


TFBLe $0-x \times 1 \times$

ZEBRA-3 70-GROUP ZONE FISSION FRACTIONS

\begin{tabular}{|c|c|c|c|c|}
\hline ous & $\begin{array}{r}\text { Enwrgy } \\
\text { Boundory } \\
\text { InvI }\end{array}$ & $\begin{array}{l}\text { Lethor } \\
\text { Hindl }\end{array}$ & Core & inflector \\
\hline 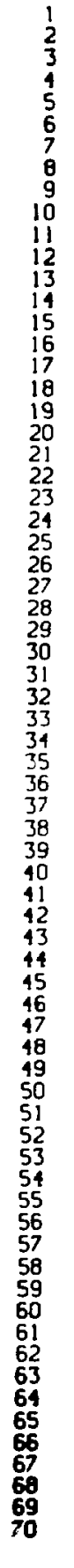 & 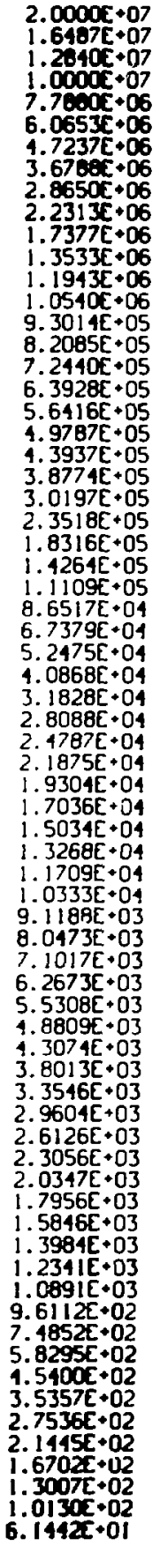 & 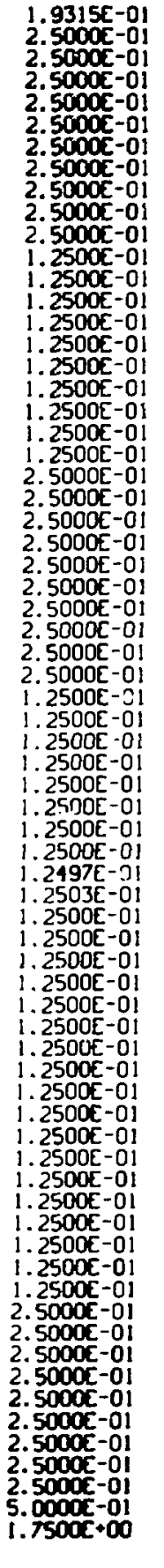 & 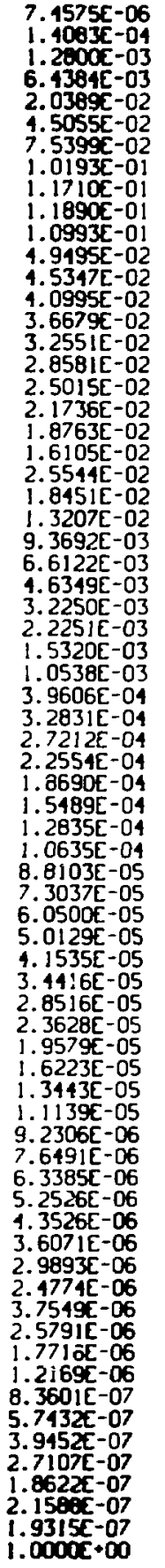 & $\begin{array}{l}5.4281 E-06 \\
1.1037 E-01 \\
1.0607 E-03 \\
5.5801 E-03 \\
1.8321 E-02 \\
4.1679 E-02 \\
7.1398 E-02 \\
9.8318 E-02 \\
1.1473 E-01 \\
1.1802 E-01 \\
1.1030 E-01 \\
5.0045 E-02 \\
4.6162 E-02 \\
4.1960 E-02 \\
3.7727 E-02 \\
3.3700 E-02 \\
2.9668 E-02 \\
2.6176 E-02 \\
2.2882 E-02 \\
1.9854 E-02 \\
1.7093 E-02 \\
2.7210 E-02 \\
1.9788 E-02 \\
1.4296 E-02 \\
1.0241 E-02 \\
7.2739 E-03 \\
5.1131 E-03 \\
3.5495 E-03 \\
2.4335 E-03 \\
1.6648 E-03 \\
1.1386 E-03 \\
4.2623 E-04 \\
3.5248 E-04 \\
2.9151 E-04 \\
2.4110 E-01 \\
1.9940 E-04 \\
1.6494 E-04 \\
1.3644 E-04 \\
1.1286 E-04 \\
9.3353 E-05 \\
7.7276 E-05 \\
6.3922 E-05 \\
5.2895 E-05 \\
4.3773 E-05 \\
3.6228 E-05 \\
2.9985 E-05 \\
2.4819 E-05 \\
2.0546 E-05 \\
1.7008 E-05 \\
1.4082 E-05 \\
1.1659 E-05 \\
9.6540 E-06 \\
7.9943 E-06 \\
6.6196 E-06 \\
5.4812 E-06 \\
4.5388 E-06 \\
3.7580 E-05 \\
3.1127 E-06 \\
2.5784 E-06 \\
3.9060 E-06 \\
2.6802 E-06 \\
1.8390 E-06 \\
1.2615 E-05 \\
0.6615 E-07 \\
5.9432 E-07 \\
4.0760 E-07 \\
2.7994 E-07 \\
1.92004 E-07 \\
2.2254 E-07 \\
1.9095 E-07 \\
1.0000 E+00\end{array}$ \\
\hline
\end{tabular}


TABLE $B-x \times x$

ZEBRA-3 25-GROUP TRANSPORT THEORY SPECTRA

\begin{tabular}{|c|c|}
\hline Ecomo & $\begin{array}{r}\text { Energy } \\
\text { Boundory } \\
\text { (eV) }\end{array}$ \\
\hline $\begin{array}{c}1 \\
2 \\
3 \\
4 \\
5 \\
6 \\
7 \\
8 \\
9 \\
10 \\
11 \\
12 \\
13 \\
14 \\
15 \\
16 \\
17 \\
18 \\
19 \\
20 \\
21 \\
22 \\
23 \\
21 \\
25\end{array}$ & $\begin{array}{l}2.0000 E+07 \\
6.0653 E+06 \\
3.6788 E+06 \\
2.2313 E+06 \\
1.3533 E+06 \\
8.2085 E+05 \\
4.9787 E+05 \\
3.0197 E+05 \\
1.8316 E+05 \\
1.1109 E+05 \\
6.7379 E+04 \\
4.0868 E+04 \\
2.1787 E+04 \\
1.5034 E+04 \\
9.1188 E+03 \\
5.5308 E+03 \\
3.3546 E+03 \\
2.0347 E+03 \\
1.2341 E+03 \\
7.2852 E+02 \\
4.5400 E+02 \\
2.75365+02 \\
1.6702 t+02 \\
1.0130 E+02 \\
6.1442 E+01 \\
1.0677 E+01\end{array}$ \\
\hline
\end{tabular}

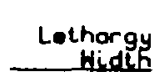

$1.1931 E+00$

5. $0000 \mathrm{E}-01$

5. $0000 \mathrm{C}-01$

5. $0000 \mathrm{E}-01$

5. $0000 \mathrm{c}-01$

5. $0000 \mathrm{E}-0 \mathrm{t}$

5. 0000L-01

5. $0000 \mathrm{C}-01$

5. $0000 \mathrm{r}-01$

5. $0000 \mathrm{E}-01$

5. $0000 \mathrm{c}-01$

5. 0000c-0!

5. $0000 \mathrm{E}-0 \mathrm{t}$

5. $0000 \mathrm{E}-01$

5. $0000 \mathrm{C}-01$

5. $0000 \mathrm{C}-01$

5. $0000 \mathrm{C}-01$

5. $0000 \mathrm{C}-01$

$5.0000 E-01$

5. $0000 \mathrm{t}-01$

5. O000E-01

5. O000E-01

5. 0000E-DI

5. 0000E-01

$1.7500 \mathrm{E}+00$

Reactor
Fiseion
rractions
$2.7780 E-02$
$1.1935 E-01$
$2.1814 E-01$
$2.2875 E-01$
$1.7302 E-01$
$1.0856 E-01$
$6.0973 E-02$
$3.2021 E-02$
$1.6211 E-02$
$7.9801 E-03$
$3.808 .2 E-03$
$1.7990 E-03$
$8.4806 E-04$
$3.9944 E-04$
$1.8811 E-04$
$6.8606 E-05$
$1.1750 E-05$
$1.9678 E-05$
$9.2779 E-06$
$4.3759 E-06$
$2.0611 E-06$
$9.7379 E-07$
$1.5949 E-07$
$2.1687 E-07$
$1.9402 E-07$
$1.0000 E-00$

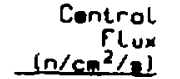

$4.7735 E-03$

$2.0631 \mathrm{E}-02$

4. 1355E-02

$5.7121 E-02$

9.5572E-02

1. $5932 \mathrm{E}-01$

j. $7883 t-01$

1. 4809 -0

i. $0738 E-01$

8. 3008E-02

$5.6679 \mathrm{E}-02$

2.7896E-02

1. 3885E-02

3. $8037 \mathrm{E}-03$

1. $1282 \mathrm{E}-03$

$3.8974 E-04$

9. $0: 85 \mathrm{E}-05$

3. $8131 \mathrm{E}-05$

8. 5044E-06

3. 4457E-06

$7.2318 E-07$

2. 1049E-07

9. 2897E-08

2. $6 \mathrm{~B} 16 \mathrm{E}-0 \mathrm{~B}$

2.3378E-OB

i. $0000 \mathrm{E} \cdot 00$

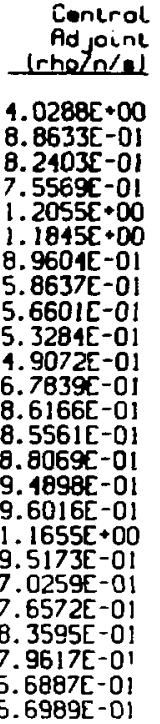

5. 6989 E-01

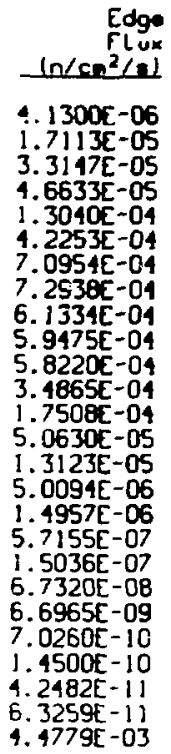

Fd JoLne Irhotn/sil

3. $1748 E-02$

6. $31905-03$

5. $2055 E-03$

4. $1542 E-03$

5. $7553 E-03$

5. $88905-03$

2. 88945-03

1. $12745-03$

$7.4367 E-04$

$5.5176 E-04$

$4.40425-04$

4.8997E-04

6.0921E-04

6. 0848E-04

6.7095E-04

7. $65695-04$

9. $384 \mathrm{EE}-04$

$1.3292[-03$

$1.0122 E-0.3$

$9.2703 \mathrm{E}-04$

9.47335 .04

1.0070E-03

Q.7713E-

$7.1506 \mathrm{E}-0$

$1.13986-03$

TABLE $B-x \times x \mid$

ZEBRA-3 25-GROUP ZONE FISSION FRACTIONS

\begin{tabular}{|c|c|c|c|c|}
\hline Oup & $\begin{array}{r}\text { Energy } \\
\text { Boundory } \\
\text { (nv) }\end{array}$ & $\begin{array}{l}\text { Lethorgy } \\
\text { Hidith }\end{array}$ & Core & Reflecto \\
\hline $\begin{array}{r}1 \\
2 \\
3 \\
1 \\
5 \\
6 \\
7 \\
8 \\
9 \\
10 \\
11 \\
12 \\
13 \\
14 \\
15 \\
16 \\
17 \\
18 \\
19 \\
20 \\
21 \\
22 \\
23 \\
24\end{array}$ & 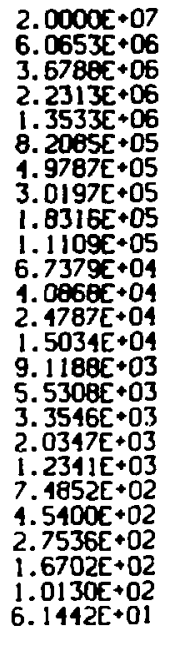 & $\begin{array}{l}1.1931 E+00 \\
5.0000 E-0 i \\
5.0000 E-01 \\
5.0000 E-01 \\
5.0000 E-01 \\
5.0000 E-01 \\
5.0000 E-01 \\
5.0000 E-01 \\
5.0000 E-01 \\
5.0000 E-01 \\
5.0000 E-01 \\
5.0000 E-01 \\
5.0000 E-01 \\
5.0000 E-01 \\
5.0000 E-01 \\
5.0000 E-01 \\
5.0000 E-01 \\
5.0000 E-01 \\
5.0000 E-01 \\
5.0000 E-01 \\
5.000 E-01 \\
5.0000 E-01 \\
5.0000 E-01 \\
5.0000 \\
1.7500 E+01 \\
\end{array}$ & $\begin{array}{l}2.8256 E-02 \\
1.2045 E-01 \\
2.1903 E-01 \\
2.2883 E-01 \\
1.7252 E-01 \\
1.0788 E-01 \\
6.0412 E-02 \\
3.1658 E-02 \\
1.5981 E-02 \\
7.8599 E-03 \\
3.7572 E-03 \\
1.7782 E-03 \\
8.3945 E-04 \\
3.3594 E-04 \\
1.8656 E-04 \\
8.7946 E-05 \\
4.1462 E-05 \\
1.9551 E-05 \\
9.2217 E-06 \\
4.3507 E-06 \\
2.0529 E-06 \\
9.6884 E-07 \\
4.5729 E-07 \\
2.1588 E-07 \\
1.9315 E-07 \\
1.0000 E+00\end{array}$ & $\begin{array}{l}2.5078 E-02 \\
1.130 E \mathrm{E}-01 \\
2.1308 \mathrm{E}-01 \\
2.2832 \mathrm{E}-01 \\
1.7589 \mathrm{E}-01 \\
1.1243 \mathrm{E}-01 \\
6.4157 \mathrm{E}-02 \\
3.4085 \mathrm{~L}-02 \\
1.7515 \mathrm{E}-02 \\
8.6626 \mathrm{E}-03 \\
4.0993 \mathrm{E}-03 \\
1.9173 \mathrm{E}-03 \\
8.9695 \mathrm{E}-04 \\
1.1993 \mathrm{E}-04 \\
1.9682 \mathrm{E}-04 \\
9.2358 \mathrm{E}-05 \\
4.3389 \mathrm{E}-05 \\
2.0398 \mathrm{E}-05 \\
9.5971 \mathrm{E}-06 \\
4.5191 \mathrm{E}-06 \\
2.1277 \mathrm{E}-06 \\
1.0019 \mathrm{E}-06 \\
4.7198 \mathrm{E}-07 \\
2.2254 \mathrm{E}-07 \\
1.9895 \mathrm{E}-07 \\
1.0000 \mathrm{E}-00\end{array}$ \\
\hline
\end{tabular}


TABLE $B-\times \times \times 11$

600IVA 70-EROUP TRRWSPORT THEORY SPECT ${ }^{-} A$

\begin{tabular}{|c|c|}
\hline froug & $\begin{array}{r}\text { Energy } \\
\text { Boundory } \\
\text { InVI }\end{array}$ \\
\hline 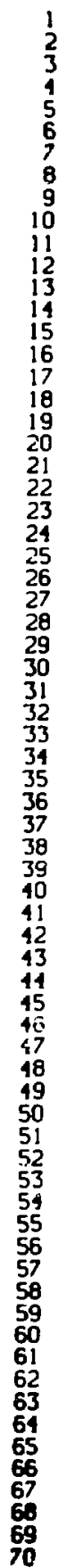 & 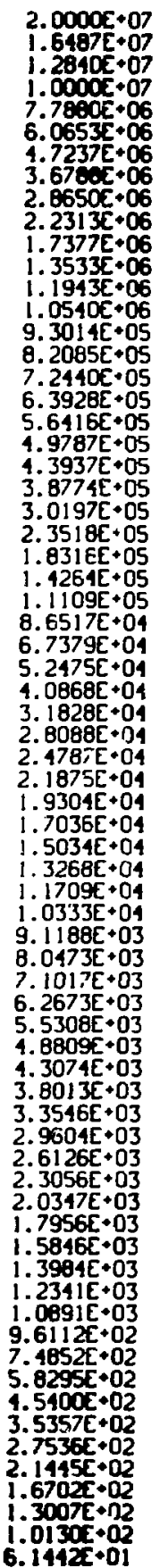 \\
\hline
\end{tabular}

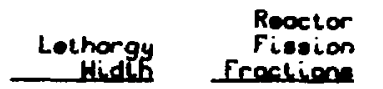

$1.9315 E-01$

2.5000E-0

2. $5000 \mathrm{~L}-01$

$2.5000 \mathrm{E}-01$

2. $5000 \mathrm{c}-01$

$2.5000 \mathrm{C}-0$

$2.5000 \mathrm{c}-01$

$2.5000 \bar{c}-0$

2. $5000 \mathrm{c}-01$

2. 5000E-01

2. $5000 E-01$

1. $2500 \mathrm{E}-01$

$1.250 r^{-5}-01$

$1.2500 \bar{\varepsilon}-0$

1. 2500E-01

$1.2500 \mathrm{E}-01$

$1.25005-01$

$1.25005-01$

$1.25005-01$

$1.2500 \mathrm{E}-01$

1. 2500E-01

$2.5000 \mathrm{C}-01$

$2.5000 \mathrm{E}-01$

2. 5000E-01

2. $5000 \mathrm{E}-01$

2. 5000E-01

2. 5000E-01

$2.5000 E-01$

2. 5000E-01

2. 5000E-01

2. 5000E-01

1. 2500E-01

$1.2500 \mathrm{E}-01$

$1.25005-01$

j. $2500 E-01$

1. 2500E-01

1. 2500 E -01

1. $2500 \mathrm{E}-01$

1. $25005-01$

1. $2497 E-01$

$1.2503 E-01$

$1.2500 E-0$

1.2500 -01

$1.2500 E-01$

$1.2500 E-01$

$1.2500 \mathrm{E}-01$

1. 2500E-0

1. $2500 \mathrm{E}-0$

1. $2500 \mathrm{t}-0$

$1.2500 t-01$

$1.2500 \mathrm{E}-01$

1. $2500 \mathrm{E}-0$

1 . 2500 -0

$1.25005-01$

$1.2500 \mathrm{E}-01$

1. $2500 \mathrm{E}-01$

1. $2500 \mathrm{E}-01$

i. 25005-01

1. $2500 \mathrm{C}-01$

2.50005-0

$2.5000 \mathrm{r}-01$

2. $5000 \mathrm{E}-01$

2. $5000 \mathrm{E}-01$

2.5000E-01

2. $5000 \mathrm{E}-01$

2. $5000 \mathrm{c}-01$

2. $5000 \mathrm{c}-0$

2. $5000 \mathrm{C}-01$

5.0000E-01

$1.7500 \mathrm{C} \cdot 00$

$7.51135-06$

1. 3537E-04

$1.2037 \varepsilon-03$

$6.0041 E-03$

$1.9026 E-02$

$4.2329 \mathrm{c}-02$

$7.1590-02$

9.8051E-02

1. |42IE-0|

1. $1014 E-01$

$4.6150 \mathrm{E}-02$

4. 1981E-02

3. $7822 \mathrm{E}-02$

$3.3754 E-02$

2. $9755 E-02$

2. $6155 \mathrm{E}-02$

2. $2835 \mathrm{E}-02$

$1.9779 E-02$

$1.7054 E-02$

$2.7146 \mathrm{E}-02$

1. $9708 \mathrm{E}-02$

$1.4138 E-02$

$1.0104 E-02$

$7.1135 E-03$

3. 481 OE-03

$2.4034 E-03$

1. $6556 \mathrm{E}-03$

1. $1391 \mathrm{E}-03$

$4.2816 \mathrm{E}-04$

$3.5494 \mathrm{E}-04$

2. $9421 \mathrm{E}-04$

$2.4385 \mathrm{E}-04$

2. 0208E-04

1. $6746 E-04$

1. 3877E-04

1. $14985-04$

9.5249E-05

7. $8959 \mathrm{E}-05$

$6.5403 E-05$

5. $41895-05$

4. 4898E-05

3. $7200 \mathrm{E}-05$

$3.0822 E-05$

$2.5537 \mathrm{E}-05$

2. $1160 \mathrm{r}-05$

1. $7532 \mathcal{E}-05$

$1.4527 \varepsilon-05$

$1.2037 \mathrm{E}-05$

9. $9742 \mathcal{E}-05$

8. $2650 E-06$

6. 8486E-06

5. 6752E-06

$4.7027 E-05$

3. $8973 \mathrm{E}-06$

3. $2297 \mathrm{E}-06$

2. $6766 E-06$

4.0564E-06

2.7661E-06

$1.91385-06$

1.3146E-06

9.0310E-07

$6.2044 E-07$

4.2626E-07

$2.9205 \mathrm{E}-07$

2.0122E-07

2. 3327 -07

2. 0065:-07

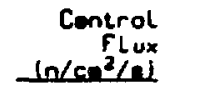

1. $1756 \mathrm{E}-01$

5.0064E-02

5. $0005 E-03$

1 . 0000 -00
. 73665-06

8. 3090 -05

7. $6020 \mathrm{~F}-04$

$3.8733 t-03$

$1.2082 E-02$

2.6124E-02

$4.4723 \mathrm{E}-02$

$6.40885-02$

7.9928E-0

8.8377 $\mathrm{E}-0$

9. $1587 \mathrm{E}-02$

4. $5580 \mathrm{E}-02$

4. $4699 E-02$

4. 3384E-02

4. $2222 \mathrm{E}-02$

4. 0821E-02

3. $9088 \mathrm{E}-02$

$3.8109 \mathrm{E}-02$

$3.6137 \mathrm{E}-02$

3. $3619 E-02$

3. $09+2 E-02$

$5.2726 \mathrm{E}-02$

4. $08205-02$

$3.0303 E-02$

2.1991E-02

1.55895-02

1. $1008 \mathrm{E}-02$

7. 5989 -03

5. 0071E-03

3. 3791E-03

2. $0980 \mathrm{E}-03$

7.051 3E-04

5.6507E-04

4. $2427 E-04$

3. $1081 E-04$

$2.7003 E-04$

$2.1795 \varepsilon-04$

1. 6529E-04

1. $3103 E-04$

9.936日E-05

$7.6688 \mathrm{E}-05$

5. 7805E-05

4. 3791E-05

3. 3774E-05

2. $4951 E-05$

1. $9964 E-05$

$1.5613 E-05$

$1.1947 E-05$

$1.0223 E-05$

$7.4913 E-06$

6. $0601 E-06$

4. $6769 \mathrm{C}-06$

3. $5714 \mathrm{E}-06$

2. 69695-06

2. $0668 \mathrm{E}-06$

1.8177E-06

1.2764E-06

9.3625E-07

8. 1954E-07

$1.12785-06$

6.5535 -07

3.7751E -07

2. 8996 -07

1. 8848c-07

$1.0295 \mathrm{E}-07$

$7.4411 \mathrm{E}-0 \mathrm{O}$

5.0942 -00

$3.460 x-00$

3. $85755-00$

$1.7697 \mathrm{E}-08$

1. $0000 \mathrm{C}+00$

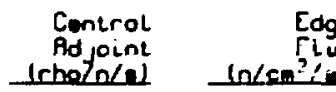

2. $1307 \mathrm{E}+00$

2. $0079 \varepsilon+00$

$1.6723 E+00$

$1.5675 E+00$

$1.3105 E+00$

$1.0052 E+00$

9.9921E-01

$1.0060 E+00$

$1.0126 E+00$

$1.0007 E+00$

9.6885E-01

9. $4479 \mathrm{c}-01$

9. 4008 $-\mathrm{Cl}$

9. $40795-01$

9. 2904E - al

9. 2965E-01

9. 340BE-0I

9. $3787 E-01$

9. $5271 E-01$

9. $7032 \mathrm{E}-0$ ।

9. $8874 E-01$

$1.0148 E+00$

$1.0475 E+00$

$1.0897 E+00$

$1.1356 \mathrm{E}+00$

i. $1759 \mathrm{E} \cdot 00$

$1.2066 E+60$

$1.2422 E+00$

$1.27345 \cdot 00$

$1.2747 E+00$

1. $2966 E+00$

1. $3225 \mathrm{E}+00$

1. $3251 E+00$

$1.33305+00$

1. $3322 \mathrm{E}+00$

$1.3765 \mathrm{E} \cdot 00$

$1.35895+0 \mathrm{~s}$

$1.4093 E+10$

$1.377 \theta^{\circ}+00$

$1.402 \%$ E 00

$1.4=005 \cdot 00$

1. $3821 E+00$

$\$ .4187 \mathrm{E}+00$

$1.4785+00$

$1.4626 E+00$

$1.5600 c+00$

$1.5466 E \cdot 00$

$1.59605 \cdot 00$

$1.6140 \mathrm{E}+00$

$1.6143 E+0 G$

$1.6533 E \cdot 00$

$1.6135 E+00$

1. $5105 \mathrm{E}+00$

$1.54625+00$

$1.6275 \mathrm{C} \cdot 00$

$1.5590 \mathrm{C} \bullet 00$

$1.63795 \cdot 00$

$1.6114 E+00$

$1.3904 E+00$

$1.4726 E+00$

1. $6007 \mathrm{E} \cdot 00$

1.7 is 4 E +00

$1.6235 \mathrm{E} \cdot 00$

$1.545 \mathrm{IE} \cdot 00$

$1.6239 \mathrm{r}+00$

$1.50055+00$

$1.47815+00$

$1.4545 E+00$

$1.5190=00$

$1.69255 \cdot 00$ 
IFBLE B-YXXI11

GOOIYA 25-GROUP TRANSPORT THEORY SPECTRA

\begin{tabular}{|c|c|}
\hline Acang & $\begin{array}{r}\text { Energy } \\
\text { Boundory } \\
\text { ley }\end{array}$ \\
\hline $\begin{array}{r}1 \\
2 \\
3 \\
4 \\
5 \\
6 \\
7 \\
8 \\
9 \\
10 \\
11 \\
12 \\
13 \\
14 \\
15 \\
16 \\
17 \\
18 \\
19 \\
20 \\
21 \\
22 \\
23 \\
24\end{array}$ & $\begin{array}{l}2.0000 E+07 \\
6.0653 E+06 \\
3.6788 E+06 \\
2.2313 E+06 \\
1.3533 E+05 \\
8.2085 E+05 \\
4.9787 E+05 \\
3.0197 E+05 \\
1.8316 E+05 \\
1.1109 E+05 \\
6.7379 E+04 \\
4.0868 E+04 \\
2.1787 E+04 \\
1.5034 E+04 \\
9.1188 E+03 \\
5.5308 E+03 \\
3.3546 E+03 \\
2.0317 E+03 \\
1.2311 E+03 \\
7.4852 E+02 \\
4.5400 E+02 \\
2.7536 E+02 \\
1.6702 E+02 \\
1.0130 E+02 \\
6.1442 E+01 \\
1.0677 E+01\end{array}$ \\
\hline
\end{tabular}

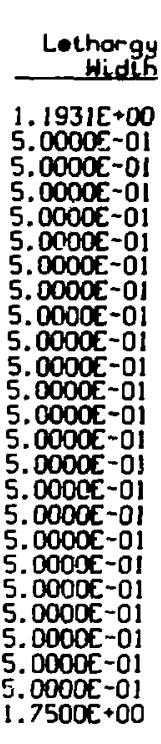

\begin{tabular}{r} 
Reactor \\
Fiseion \\
Froctione \\
\hline $2.6377 E-02$ \\
$1.1393 E-01$ \\
$2.1226 E-01$ \\
$2.2770 E-01$ \\
$1.7602 E-01$ \\
$1.1250 E-01$ \\
$6.3979 E-02$ \\
$3.3846 E-02$ \\
$1.7218 E-02$ \\
$8.4814 E-03$ \\
$4.0590 E-03$ \\
$1.9222 E-03$ \\
$9.0760 E-04$ \\
$4.2795 E-04$ \\
$2.0169 E-04$ \\
$9.5051 E-05$ \\
$4.4803 E-05$ \\
$2.1124 E-05$ \\
$9.9627 E-06$ \\
$1.6999 E-06$ \\
$2.2177 E-05$ \\
$1.0467 E-06$ \\
$4.9409 E-07$ \\
$2.3327 E-07$ \\
$2.0869 E-07$ \\
$1.0000 E+00$
\end{tabular}

$\begin{array}{r}\text { Centrol } \\ \text { Flux } \\ \text { In/cm } / \mathrm{c}^{2}\end{array}$
$1.6804 E-02$
$7.0846 E-02$
$1.4402 E-01$
$1.7996 E-01$
$1.7566 E-01$
$1.5415 E-01$
$1.1729 E-01$
$7.1123 E-02$
$3.7580 E-02$
$1.8607 E-02$
$8.3862 E-03$
$3.3662 E-03$
$1.2531 E-03$
$4.7237 E-04$
$1.6032 E-04$
$5.7746 E-05$
$2.1800 E-05$
$7.8477 E-06$
$2.8836 E-06$
$1.0329 E-06$
$4.7846 E-07$
$1.7736 E-07$
$8.5544 E-08$
$3.8575 E-08$
$1.7697 E-08$
$1.0000 E+00$

\begin{tabular}{|c|}
\hline $\begin{array}{r}\text { Conc } \\
\text { Rd jo } \\
\text { Irhoo }\end{array}$ \\
\hline $\begin{array}{l}3.1389 E+0 \\
7.2372 E-0 \\
7.2921 E-0 \\
7.1151 E-0 \\
1.3561 E+0 \\
1.3562 E+0 \\
1.0743 E+0 \\
7.7207 E-0 \\
8.3503 E-0 \\
8.8466 E-0 \\
9.2051 E-0 \\
1.4249 E+00 \\
1.9510 E+0 \\
2.0266 E+00 \\
2.0632 E+00 \\
2.2819 E+00 \\
2.3090 E+00 \\
2.3014 E+00 \\
1.6164 E+00\end{array}$ \\
\hline
\end{tabular}

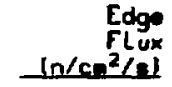

3. $8591 E-03$

$1.5475 \mathrm{E}-02$

$3.05295-02$

$3.7460 \mathrm{E}-02$

3. $6074 E-02$

3. 0205E-02

2. $1544 \mathrm{E}-02$

1. $2238 \mathrm{c}-02$

5. $9999 \mathrm{z}-03$

$2.7605 E-03$

i. $1505-03$

4. $63535-04$

1.7076E-GA

6. $4423 E-05$

2. $1923 \mathrm{t}-05$

$7.9694 E-06$

3. 0557E-06

1. $1326 \mathrm{E}-06$

4. 3067E-07

1. $6354 \mathrm{E}-07$

$7.6237 \mathrm{E}-08$

$3.0166 \mathrm{E}-08$

$1.4312 \mathrm{E}-08$

$6.25295-09$

2.7125E-09
Edge Ad joint Irhotolel

7. 4517E-01 1. 5924E-0! 1.554.4E-0: i. $4851 \mathrm{E}-0$ ! 2.7715 E 2.6347E-01 1.96685-01 1. 32265-01 $1.3352 E-01$ 1. 3347E-01 1. $3374 E-01$ 2. 0192E-01 $2.74425-01$ 2. $8559 \mathrm{E}-01$ 2. $9227 \mathrm{E}-01$ 3. $2684 \hat{E}-0$ ! 3. 3510t-0 3. $4332 \mathrm{C}-\mathrm{O}$ 2. $4845 \mathrm{E}-01$

$1.9605 E-01$

$1.8663 \bar{E}-0$ l

$1.9598 \mathrm{E}-01$

$1.8064 \hat{E}-01$

9. $0670 \mathrm{C}-02$

$9.6767 €-02$ 
TRELE $\theta-x \times x] V$

VERA-18 70-GROU TARASPOAT THEORY SPECTRA

\begin{tabular}{|c|c|c|c|c|c|c|c|}
\hline Grown & $\begin{array}{r}\text { Energy } \\
\text { Boundory } \\
\text { leYI }\end{array}$ & $\begin{array}{r}\text { Lothorgy } \\
\text { Mindih }\end{array}$ & $\begin{array}{r}\text { Reoctor } \\
\text { Fiseion } \\
\text { Iractione }\end{array}$ & 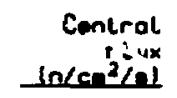 & $\begin{array}{r}\text { Centrol } \\
\text { Ad joint } \\
\text { tatentel }\end{array}$ & $\begin{array}{r}\text { F.dge } \\
F l \text { ux } \\
\ln \left\langle\mathrm{ces}^{2} /{ }^{2}\right|\end{array}$ & $\begin{array}{r}\text { Edge } \\
\text { Ad jount } \\
\text { lenotolal }\end{array}$ \\
\hline $\begin{array}{l}1 \\
2 \\
3 \\
4 \\
5 \\
6 \\
7 \\
8 \\
9 \\
10 \\
11 \\
12 \\
13 \\
14 \\
15 \\
16 \\
17 \\
18 \\
19 \\
20 \\
21 \\
22 \\
23 \\
21 \\
25 \\
26 \\
27 \\
28 \\
29 \\
30 \\
31 \\
32 \\
33 \\
34 \\
35 \\
36 \\
37 \\
38 \\
39 \\
40 \\
41 \\
42 \\
43 \\
41 \\
45 \\
46 \\
47 \\
48 \\
49 \\
50 \\
51 \\
52 \\
53 \\
54 \\
55 \\
56 \\
57 \\
58 \\
59 \\
60 \\
61 \\
62 \\
63 \\
67 \\
69 \\
70\end{array}$ & $\begin{array}{l}2.0000 E+07 \\
1.6487 E+07 \\
1.2840 E+07 \\
1.0000 E+07 \\
7.7880 E+06 \\
6.0653 E+06 \\
1.7237 E+06 \\
3.6788 E+06 \\
2.6650 E+06 \\
2.2313 E+06 \\
1.7377 E+05 \\
1.3533 E+06 \\
1.1943 E+06 \\
1.0540 E+06 \\
9.3014 E+05 \\
6.2085 E+05 \\
7.2440 E+05 \\
6.3928 E+05 \\
5.6416 E+05 \\
4.9787 E+05 \\
1.3937 E+05 \\
3.8774 E+05 \\
3.0197 E+05 \\
2.3518 E+05 \\
1.9316 E+05 \\
1.4264 E+05 \\
1.1109 E+05 \\
0.6517 E+04 \\
6.7379 E+C 4 \\
5.2475 E+04 \\
4.0868 E+04 \\
3.1828 E+04 \\
2.8088 E+04 \\
2.4787 E+01 \\
2.1875 E+04 \\
1.9304 E+04 \\
1.7036 E+04 \\
1.5034 E+04 \\
1.3268 E+04 \\
1.1709 E+04 \\
1.0333 E+04 \\
9.1188 E+03 \\
8.0473 E+03 \\
7.1017 E+03 \\
6.2673 E+03 \\
5.5308 E+03 \\
1.8809 E+03 \\
4.3074 E+03 \\
3.8013 E+03 \\
3.3546 E+03 \\
2.9604 E+03 \\
2.6126 E+03 \\
2.3056 E+03 \\
2.0347 E+03 \\
1.7956 E+03 \\
1.5846 E+03 \\
1.3964 E+03 \\
1.2341 E+03 \\
1.0891 E+03 \\
9.6112 E+02 \\
7.4852 E+02 \\
5.8295 E+02 \\
4.5400 E+02 \\
3.5357 E+02 \\
2.7536 E+02 \\
2.1445 E+02 \\
1.6702 E+02 \\
1.3007 E+02 \\
1.0130 E+02 \\
6.1442 E+01 \\
1.0577 E+01\end{array}$ & $\begin{array}{l}1.9315 E-01 \\
2.5000 E-01 \\
2.5000 E-01 \\
2.5000 E-01 \\
2.5000 E-01 \\
2.5000 E-01 \\
2.5000 E-01 \\
2.5000 E-01 \\
2.5000 E-01 \\
2.5000 E-01 \\
2.5000 E-01 \\
1.2500 E-01 \\
1.2500 E-01 \\
1.2500 E-01 \\
1.2500 E-01 \\
1.2500 E-01 \\
1.2500 E-01 \\
1.2500 E-01 \\
1.2500 E-01 \\
1.2500 E-01 \\
1.2500 E-01 \\
2.5000 E-01 \\
2.5000 E-01 \\
2.5000 E-01 \\
2.5000 E-01 \\
2.5000 E-01 \\
2.5000 E-01 \\
2.5000 E-01 \\
2.5000 E-01 \\
2.5000 E-01 \\
2.5000 E-01 \\
1.2500 E-01 \\
1.2500 E-01 \\
1.2500 E-01 \\
1.2500 E-01 \\
1.2500 E-01 \\
1.2500 E-01 \\
1.2500 E-01 \\
1.2500 E-01 \\
1.2497 E-01 \\
1.2503 E-01 \\
1.2500 E-01 \\
1.2500 E-01 \\
1.2500 E-01 \\
1.2500 E-01 \\
1.2500 E-01 \\
1.2500 E-01 \\
1.2500 E-01 \\
1.2500 E-01 \\
1.2500 E-01 \\
1.2500 E-01 \\
1.2500 E-01 \\
1.2500 E-01 \\
1.2500 E-01 \\
1.2500 E-01 \\
1.2500 E-01 \\
1.2500 E-01 \\
1.2500 E-01 \\
1.2500 E-01 \\
2.5000 E-01 \\
2.5000 E-01 \\
2.5000 E-01 \\
2.5000 E-01 \\
2.5000 E-01 \\
2.5000 E-01 \\
2.5000 E-01 \\
2.5000 E-01 \\
2.5000 E-01 \\
5.0000 E-01 \\
1.7500 E+00 \\
2\end{array}$ & 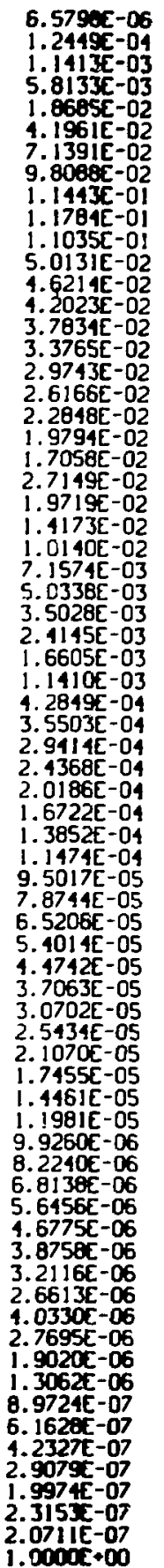 & 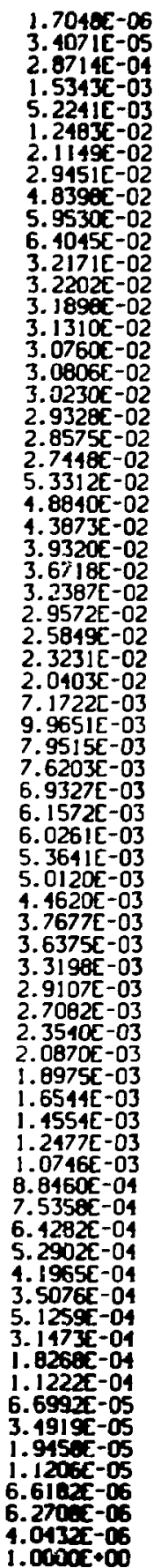 & 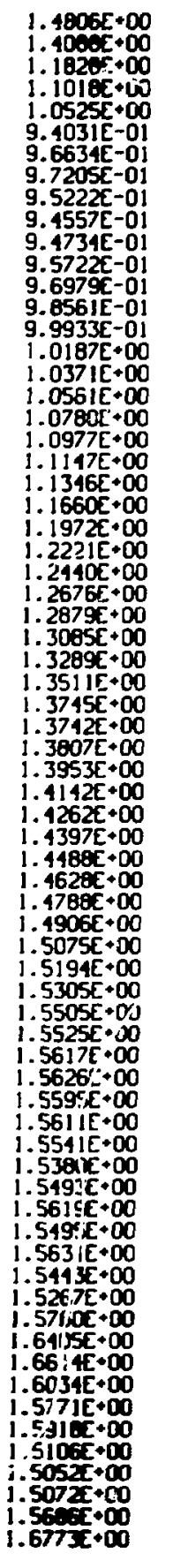 & 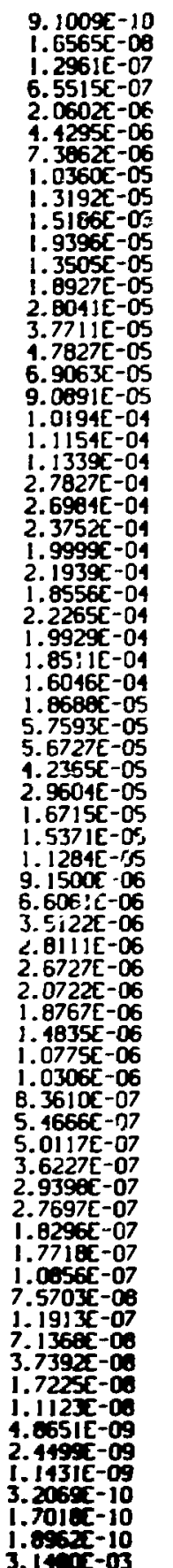 & 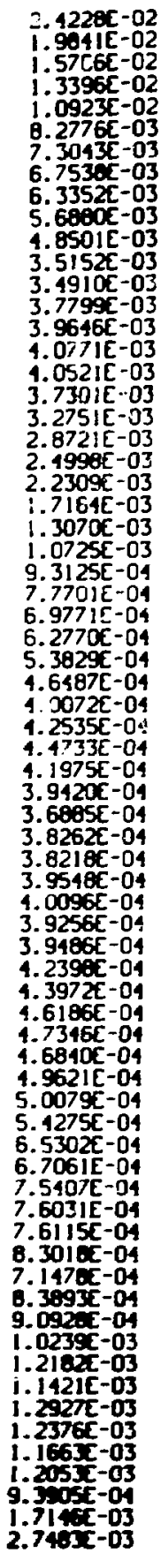 \\
\hline
\end{tabular}


Trate 0-XXXY

VERA-18 70-GROUP ZONE FISSION FRACTIONS

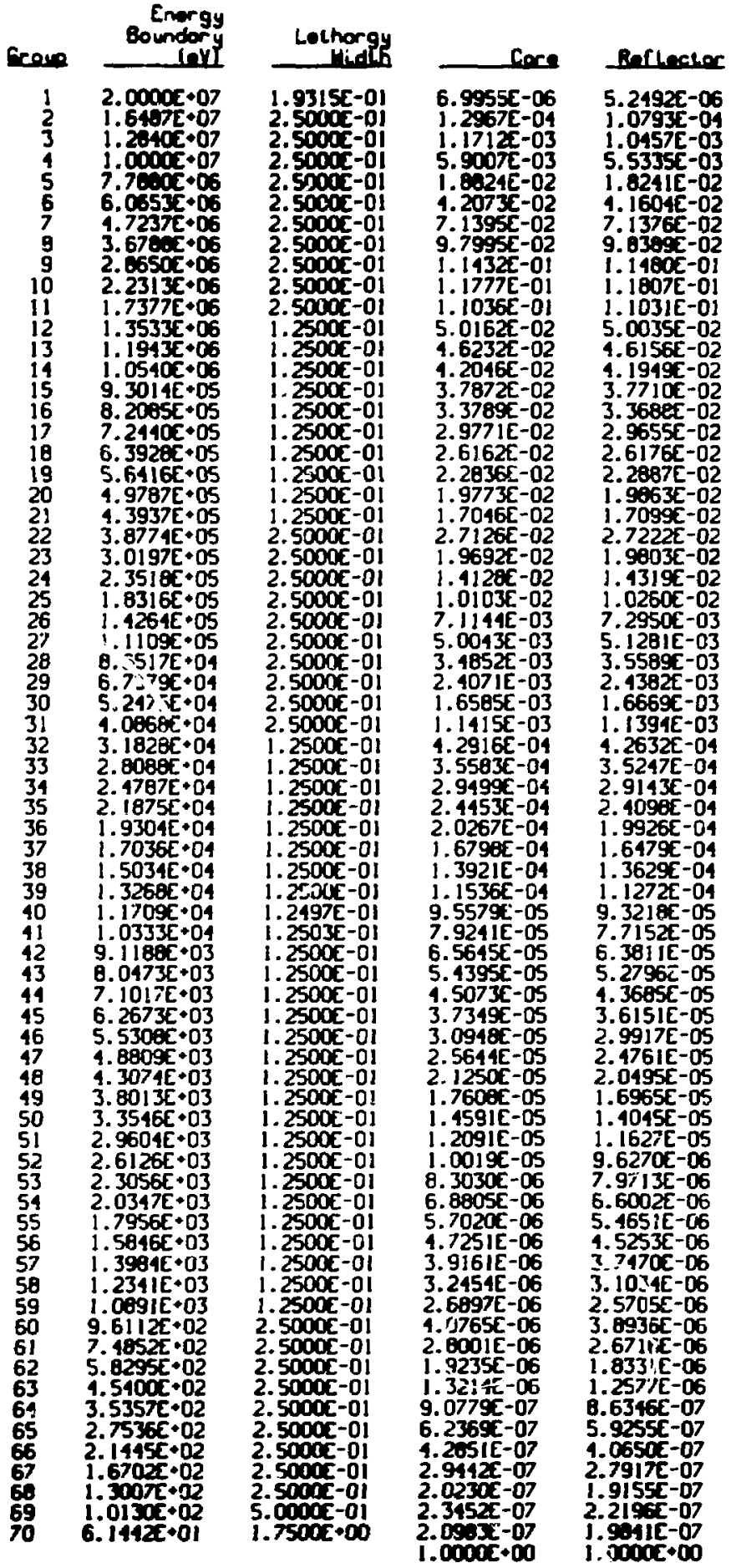


Ifoer 6-xxxyI

VERA-1R 25-GROUP TRAMSPORT THEORY SPECTPP

\begin{tabular}{|c|c|}
\hline Gcors & $\begin{array}{r}\text { Energy } \\
\text { Boundery } \\
\text { (nv) }\end{array}$ \\
\hline $\begin{array}{c}1 \\
2 \\
3 \\
4 \\
5 \\
6 \\
7 \\
8 \\
9 \\
10 \\
11 \\
12 \\
13 \\
14 \\
15 \\
16 \\
17 \\
18 \\
19 \\
20 \\
21 \\
22 \\
23 \\
24 \\
25\end{array}$ & 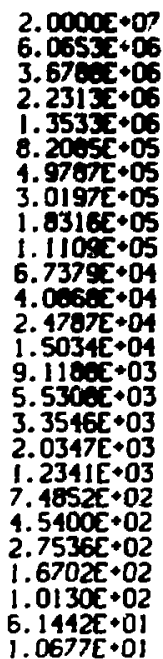 \\
\hline
\end{tabular}

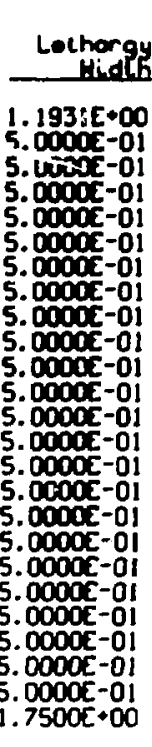

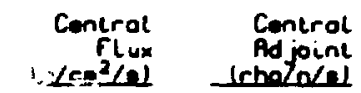

7.0013E-03

3. $3632 \mathrm{x}-02$

7.70405-02

$1.235 .5-0$

$1.275 .2-01$

$1.2112 x-01$

1. $0934 \mathrm{E}-01$

9. $2711 \varepsilon-02$

$7.6036 \mathrm{E}-02$

6. $19595-02$

4. 9001E-02

3.7510c-02

2. $65625-02$

$2.0064 \mathrm{E}-02$

$1.3636 \bar{t}-02$

9. $0467 \mathrm{E}-03$

$5.4321 E-03$

2. $8100 \mathrm{E}-03$

1. $2830 \mathrm{z}-03$

1. $9741 \mathrm{E}-04$

1.7921E-04

$5.4377 \varepsilon-05$

1. 7824[-05

6. 27005-05

4. $0432 \mathrm{E}-06$

$1.0000 \mathrm{E}+00$

2. 2504E + 00

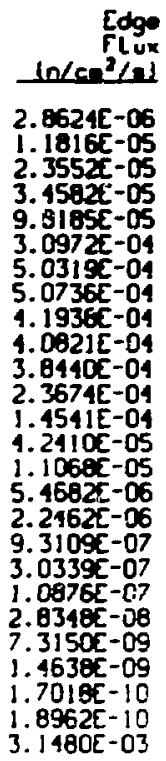

6. $0911 \mathrm{~L}-01$

$6.9548 E-01$

$6.8415 E-01$

$1.41395 \cdot 00$

$1.5144 \mathrm{E}+00$

i. $2097 \mathrm{E} \cdot 00$

e. 5413t-01

8.9131E-01

9. $2364 \mathrm{E}-01$

9.5321E-01

$1.4818 \mathrm{c} \cdot 00$

$2.02995 \cdot 00$

$2.1071 E+00$

$2.1859 \mathrm{t}+00$

2. $2507 \mathrm{E}+00$

2. $2451 \mathrm{E}+0 \mathrm{O}$

$2.2491 \mathrm{E}+00$

$1.6795 f+00$

$1.1934 E+00$

$1.1495 \mathrm{f}+00$

1. $1213 \mathrm{~F}+\mathrm{DO}$

$1.0886 \mathrm{c}+00$

5. 6695L-01
5. $0621 \mathrm{~L}-01$
Eoge irtolocel

3.0391E-02 5.6317E-03 4.73C7E-03

3. 80.7TE-03

5. $331 x-03$

5. $4700 \mathrm{c}-03$ 2.7479F-03 $1.0934 E-03$

7.2422t-04

5. $3300 \mathrm{r}-04$

$4.2142 E-04$

4. 6657 E-04

5. $69 ! 7 E-04$

5. 6427E-04

5. $9676 E-04$

6. 85685-04

$8.5555 \mathrm{C}-04$

1. $1225 E-03$

e. 9019t-04

8. $10355-04$

9. $7997 \mathrm{E}-04$

9. $6883 \mathrm{E}-04$

$7.7502 E-04$

$5.1968 E-04$

S. $9332 E-04$

IFaLE B-XXXYII VERA-18 25-GROUP ZONC FISSION FRACTIONS

\begin{tabular}{|c|c|c|c|c|}
\hline irave & $\begin{array}{r}\text { Energy } \\
\text { Boundory } \\
\text { Cerl' }\end{array}$ & $\begin{array}{r}\text { Lethorgy } \\
\text { Hidith }\end{array}$ & Core & Rerlenctor \\
\hline $\begin{array}{c}1 \\
? \\
3 \\
4 \\
4 \\
5 \\
6 \\
7 \\
8 \\
8 \\
9 \\
10 \\
11 \\
12 \\
13 \\
14 \\
15 \\
16 \\
17 \\
18 \\
19 \\
20 \\
21 \\
22 \\
23 \\
24 \\
25\end{array}$ & 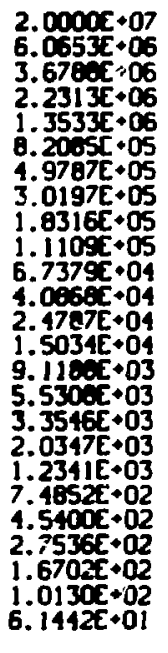 & 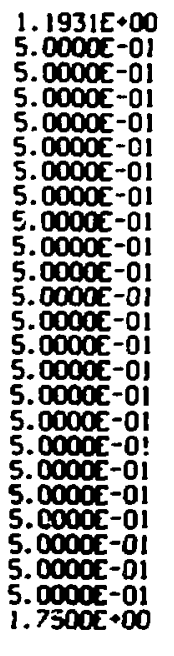 & 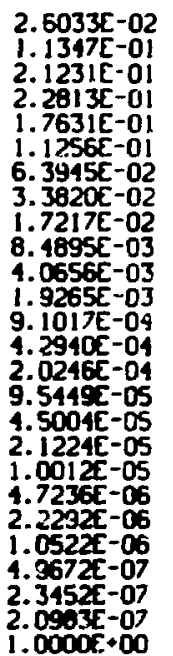 & $\begin{array}{l}2.4934 E-02 \\
1.1290 E-01 \\
2.1319 E-01 \\
2.2838 E-01 \\
1.7585 E-01 \\
1.1241 E-01 \\
6.4184 E-02 \\
3.4122 E-02 \\
1.7555 E-02 \\
8.6870 E-03 \\
1.1051 E-03 \\
1.9181 E-03 \\
8.9645 E-04 \\
4.1936 E-04 \\
1.9644 E-04 \\
9.2139 E-05 \\
4.3270 E-05 \\
2.0338 E-05 \\
9.5676 E-06 \\
1.5047 E-06 \\
2.1211 E-06 \\
9.9905 E-07 \\
1.7073 E-07 \\
2.2195 E-07 \\
1.9941 E-07 \\
1.0000 E-00\end{array}$ \\
\hline
\end{tabular}


TfQue $8-x \times x \times 111$

2PR-3-6r 70-CROUP IAFASPORT THCORY SPECTAA

\begin{tabular}{|c|c|c|c|c|c|c|c|}
\hline ang & $\begin{array}{r}\text { Enorgy } \\
\text { Boundory } \\
\text { Cayl }\end{array}$ & $\begin{array}{l}\text { Lethorgy } \\
\text { Hidilh }\end{array}$ & $\begin{array}{r}\text { Reoctor } \\
\text { Fiseion } \\
\text { Erealienn }\end{array}$ & $\begin{array}{r}\text { Control } \\
f \text { luk } \\
\left.\ln / \mathrm{en}^{2} / \mathrm{d}\right)\end{array}$ & $\begin{array}{r}\text { Centrol } \\
\text { Ad pint } \\
\text { lrboln<l }\end{array}$ & $\begin{array}{r}5 \\
\ln / s^{2}\end{array}$ & A \\
\hline $\begin{array}{l}1 \\
2 \\
3 \\
1 \\
5 \\
6 \\
7 \\
8 \\
9 \\
10 \\
11 \\
12 \\
13 \\
14 \\
15 \\
16 \\
17 \\
18 \\
19 \\
20 \\
21 \\
22 \\
23 \\
24 \\
25 \\
26 \\
27 \\
28 \\
29 \\
30 \\
31 \\
32 \\
33 \\
34 \\
35 \\
36 \\
37 \\
38 \\
39 \\
40 \\
41 \\
42 \\
43 \\
44 \\
45 \\
46 \\
47 \\
48 \\
49 \\
50 \\
51 \\
52 \\
53 \\
54 \\
55 \\
56 \\
57 \\
58 \\
59 \\
60 \\
61 \\
62 \\
63 \\
64 \\
65 \\
66 \\
67 \\
69 \\
79 \\
70\end{array}$ & 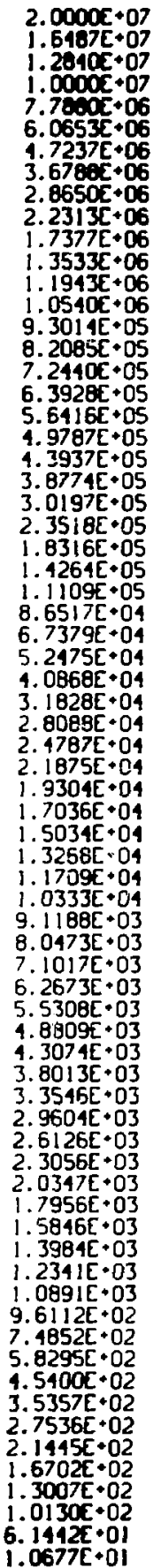 & 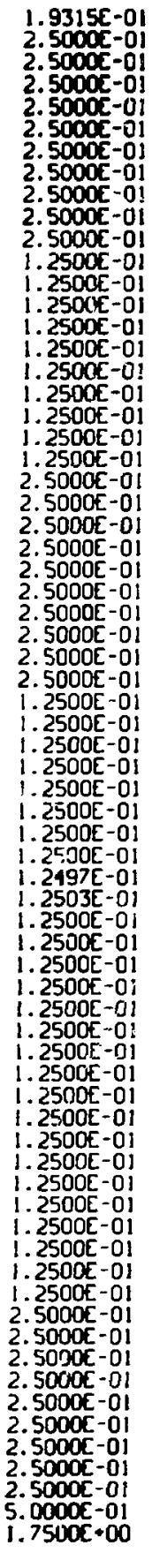 & $\begin{array}{l}5.5240 E-06 \\
1.2376 E-04 \\
1.1371 E-03 \\
5.8016 E-03 \\
1.6669 E-02 \\
1.1955 E-02 \\
7.1405 E-02 \\
9.8117 E-02 \\
1.144 E-01 \\
1.1765 E-01 \\
1.1034 E-01 \\
5.0121 E-02 \\
4.6206 E-02 \\
4.2014 E-02 \\
3.7822 E-02 \\
3.3756 E-02 \\
2.9734 E-02 \\
2.6163 E-02 \\
2.2847 E-02 \\
1.9796 E-02 \\
1.7059 E-02 \\
2.7150 E-02 \\
1.9721 E-02 \\
1.4180 E-02 \\
1.0146 E-02 \\
7.1646 E-03 \\
5.0386 E-03 \\
3.5055 E-03 \\
2.4155 E-03 \\
1.6606 E-03 \\
1.1407 E-02 \\
4.2828 E-04 \\
3.5481 E-04 \\
2.9393 E-04 \\
2.4348 E-04 \\
2.01 E 7 E-04 \\
1.6704 E-04 \\
1.7836 E-04 \\
1.1459 E-04 \\
9.4892 E-05 \\
7.8635 E-05 \\
6.5113 E-05 \\
5.3932 E-05 \\
1.4671 E-05 \\
3.7002 E-05 \\
3.0650 E-05 \\
2.5389 E-05 \\
2.1032 E-05 \\
1.7422 E-05 \\
1.4433 E-05 \\
1.1957 E-05 \\
9.9063 E-06 \\
2.2074 E-06 \\
6.7997 E-06 \\
5.6337 E-06 \\
4.6676 E-06 \\
3.8674 E-06 \\
3.2045 E-06 \\
2.6551 E-06 \\
4.0239 E-06 \\
2.7632 E-06 \\
1.8976 E-06 \\
1.3031 E-06 \\
8.9507 E-07 \\
6.1477 E-07 \\
4.2221 E-07 \\
2.9006 E-07 \\
1.9923 E-07 \\
2.3093 E-07 \\
2.0657 E-07 \\
1.0000 E+00\end{array}$ & $\begin{array}{l}1.6659 E-05 \\
3.1457 E-05 \\
2.7204 E-04 \\
1.9774 E-03 \\
4.7425 E-03 \\
1.084 E-02 \\
1.9259 E-02 \\
2.9226 E-02 \\
4.0225 E-02 \\
4.7243 E-02 \\
5.4108 E-02 \\
3.0354 E-02 \\
3.2865 E-02 \\
3.6305 E-02 \\
3.4776 E-02 \\
3.7476 E-02 \\
4.1527 E-02 \\
1.3325 E-02 \\
4.2097 E-02 \\
4.0060 E-02 \\
3.9856 E-02 \\
7.9961 E-02 \\
7.5726 E-02 \\
5.5134 E-02 \\
3.0099 E-02 \\
4.6887 E-02 \\
1.9743 E-02 \\
3.6244 E-02 \\
2.5350 E-02 \\
1.4084 E-02 \\
5.1270 E-03 \\
2.1105 E-03 \\
8.2144 E-03 \\
5.2536 E-03 \\
2.6731 E-03 \\
1.4728 E-03 \\
8.0279 E-04 \\
7.0509 E-04 \\
5.2531 C-04 \\
4.1496 E-04 \\
2.9338 E-04 \\
1.7067 E-04 \\
1.3867 E-04 \\
1.3065 E-04 \\
8.4155 E-05 \\
8.4793 E-05 \\
5.7316 E-05 \\
3.8216 E-05 \\
2.9270 E-05 \\
2.0325 E-05 \\
1.3526 E-05 \\
8.8504 E-06 \\
7.0873 E-06 \\
5 . .1481 E-06 \\
3.6681 E-06 \\
2.8033 E-06 \\
2.0845 E-06 \\
1.2983 E-06 \\
1.1993 E-06 \\
1.5552 E-06 \\
9.4150 E-07 \\
4.5198 E-07 \\
2.8317 E-07 \\
1.8854 E-07 \\
1.0036 E-07 \\
6.6812 E-00 \\
4.6483 E-08 \\
2.9093 E-08 \\
3.2059 E-08 \\
1.8054 E-08 \\
1.0000 E+00\end{array}$ & 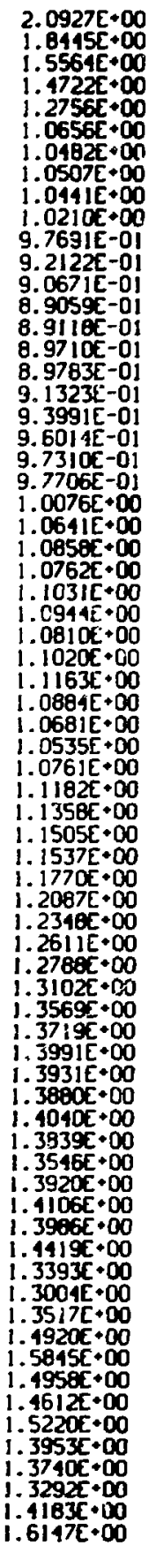 & 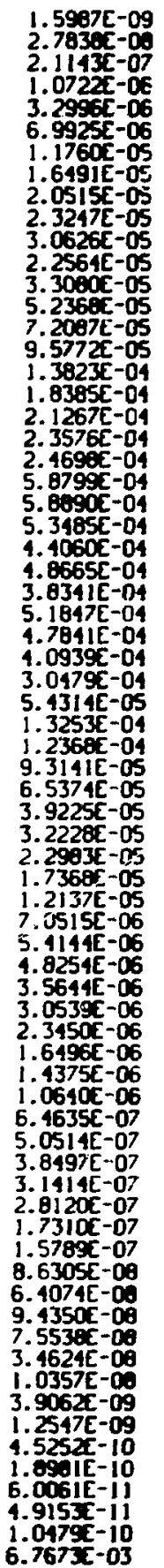 & 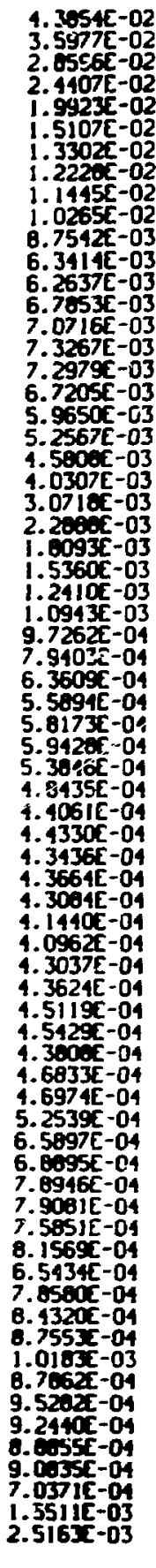 \\
\hline
\end{tabular}


Trace $B-x \times \times 1 \times$

ZPR-3-6r 70-GROUP ZONE TISSION FRACTIONS

\begin{tabular}{|c|c|c|c|c|}
\hline $\cos \theta$ & $\begin{array}{r}\text { Energy } \\
\text { Boundory } \\
\mid \text { |ry| }\end{array}$ & $\begin{array}{r}\text { Lothorgy } \\
\text { Hidith }\end{array}$ & Core & Rinflection \\
\hline $\begin{array}{l}1 \\
2 \\
3 \\
4 \\
5 \\
6 \\
7 \\
8 \\
9 \\
10 \\
11 \\
12 \\
13 \\
14 \\
15 \\
16 \\
17 \\
18 \\
19 \\
20 \\
21 \\
22 \\
23 \\
24 \\
25 \\
26 \\
27 \\
28 \\
29 \\
30 \\
31 \\
32 \\
33 \\
34 \\
35 \\
36 \\
37 \\
38 \\
39 \\
40 \\
41 \\
42 \\
43 \\
44 \\
45 \\
46 \\
47 \\
48 \\
49 \\
50 \\
51 \\
52 \\
53 \\
54 \\
55 \\
56 \\
57 \\
58 \\
59 \\
60 \\
61 \\
62 \\
63 \\
64 \\
65 \\
66 \\
67 \\
68 \\
69 \\
70\end{array}$ & 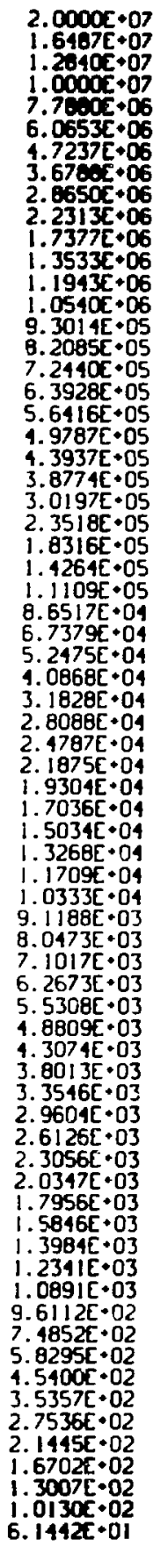 & 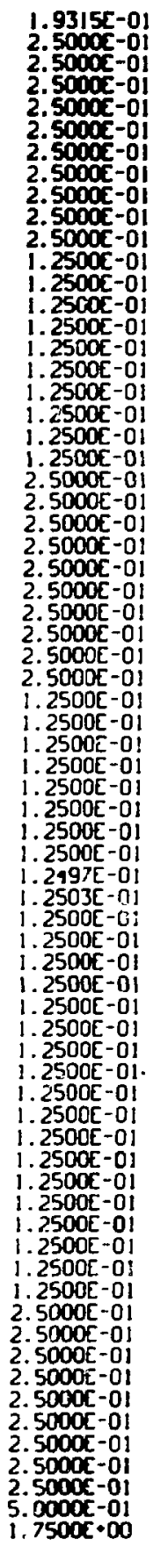 & $\begin{array}{l}6.0307 E-06 \\
1.2762 E-04 \\
1.1594 E-03 \\
5.8667 E-03 \\
1.8772 E-02 \\
4.2035 E-02 \\
7.1402 E-02 \\
9.8040 E-02 \\
1.1437 E-01 \\
1.1780 E-01 \\
1.1035 E-01 \\
5.0146 E-02 \\
4.6222 E-02 \\
4.2034 E-02 \\
3.7853 E-02 \\
3.3777 E-02 \\
2.9757 E-02 \\
2.6162 E-02 \\
2.2839 E-02 \\
1.9780 E-02 \\
1.7050 E-02 \\
2.7134 E-02 \\
1.9702 E-02 \\
1.4146 E-02 \\
1.0117 E-02 \\
7.1319 E-03 \\
5.0162 E-03 \\
3.4922 E-03 \\
2.4099 E-03 \\
1.6592 E-03 \\
1.1411 E-03 \\
4.2883 E-04 \\
3.5545 E-04 \\
2.9459 E-04 \\
2.4414 E-04 \\
2.0230 E-04 \\
1.6763 E-04 \\
1.3890 E-04 \\
1.1508 E-04 \\
9.5329 E-05 \\
7.9021 E-05 \\
6.5451 E-05 \\
5.4227 E-05 \\
4.4927 E-05 \\
3.7223 E-05 \\
3.0840 E-05 \\
2.5551 E-05 \\
2.1171 E-05 \\
1.7541 E-05 \\
1.4534 E-05 \\
1.2043 E-05 \\
9.9786 E-06 \\
8.2685 E-05 \\
6.8513 E-05 \\
5.6774 E-06 \\
4.7044 E-06 \\
3.8986 E-06 \\
3.2307 E-06 \\
2.6774 E-06 \\
4.0576 E-06 \\
2.7869 E-06 \\
1.9143 E-05 \\
1.3149 E-06 \\
9.0329 E-07 \\
6.2055 E-07 \\
4.2632 E-07 \\
2.9290 E-07 \\
2.0124 E-07 \\
2.3320 E-07 \\
2.0070 E-07 \\
1.0000 E-00\end{array}$ & 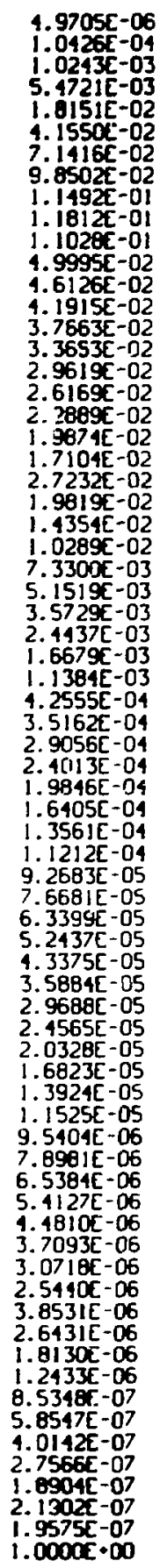 \\
\hline
\end{tabular}


TfaLe $\mathrm{B}-\mathrm{XL}$

\section{ZPR-3-6F 25-GROUP TRFWSPORT THEORY SPECIRA}

\begin{tabular}{|c|c|c|c|c|c|c|c|}
\hline ביב & $\begin{array}{r}\text { Energy } \\
\text { Boundory } \\
\text { (ay) }\end{array}$ & $\begin{array}{c}\text { Lethorgy } \\
\text { Hidilh }\end{array}$ & $\begin{array}{r}\text { Reoctor } \\
\text { fiseion } \\
\text { froctions }\end{array}$ & $\begin{array}{r}\text { Centrol } \\
\text { Flux } \\
\ln / a \operatorname{sil}^{2} / \mathrm{l}\end{array}$ & $\begin{array}{r}\text { Central } \\
\text { Ad joint } \\
\text { Inholn/nl }\end{array}$ & $\begin{array}{r}\text { Edge } \\
F \operatorname{lux} \\
\ln \left\langle a e^{2} / a\right) \\
\end{array}$ & $\begin{array}{r}\text { Edge } \\
\text { Ad jount } \\
\text { Ichotn/al }\end{array}$ \\
\hline $\begin{array}{l}1 \\
2 \\
3 \\
1 \\
5 \\
6 \\
7 \\
8 \\
9 \\
10 \\
11 \\
12 \\
13 \\
14 \\
15 \\
16 \\
17 \\
18 \\
19 \\
20 \\
21 \\
22 \\
23 \\
24 \\
25\end{array}$ & $\begin{array}{l}2.0000 E+07 \\
6.0553 E+0 . \\
3.670 E+05 \\
2.2313 E+05 \\
1.3533 E+05 \\
6.2005 E+05 \\
1.9787 E+05 \\
3.0197 E+05 \\
1.0316 E+05 \\
1.1109 E+05 \\
6.7379 E+04 \\
1.0668+04 \\
2.4787 E+04 \\
1.5034 E+04 \\
9.1186 E+03 \\
5.5300 E+03 \\
3.3516 E+03 \\
2.0347 E+03 \\
1.2341 E+03 \\
7.1852 E+02 \\
4.5400 E+02 \\
2.7536 E+02 \\
1.6702 E+02 \\
1.0130 E+02 \\
6.1442 E+01 \\
1.0677 E+01\end{array}$ & 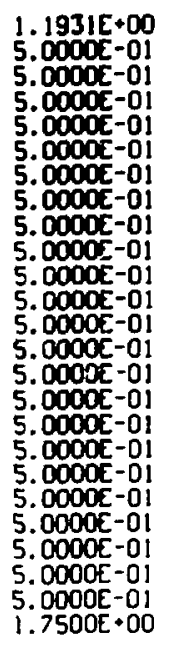 & $\begin{array}{l}2.5738 E-02 \\
1.1336 E-01 \\
2.1257 E-01 \\
2.2819 E-01 \\
1.7616 E-01 \\
1.1250 E-01 \\
6.4004 E-02 \\
3.3901 E-02 \\
1.7310 E-02 \\
8.5441 E-03 \\
4.0761 E-03 \\
1.9238 E-03 \\
9.0612 E-04 \\
4.2648 E-04 \\
2.0072 E-04 \\
9.4493 E-05 \\
4.4501 E-05 \\
2.0968 E-05 \\
9.8839 E-06 \\
4.6607 E-06 \\
2.1982 E-06 \\
1.0370 E-06 \\
4.8929 E-07 \\
2.3093 E-07 \\
2.0657 E-07 \\
1.0000 E+00\end{array}$ & $\begin{array}{l}6.5255 E-03 \\
3.0107 E-02 \\
6.9451 E-02 \\
1.0135 E-01 \\
1.3430 E-01 \\
1.6442 E-01 \\
1.5968 E-01 \\
1.3006 E-01 \\
7.6986 E-02 \\
5.7997 E-02 \\
3.9434 E-02 \\
1.5752 E-02 \\
1.0202 E-02 \\
1.9387 E-03 \\
5.2414 E-04 \\
2.0959 E-04 \\
4.9780 E-05 \\
1.3704 E-05 \\
1.0528 E-06 \\
1.3935 E-06 \\
4.7171 E-07 \\
1.6717 E-07 \\
1.5566 E-08 \\
3.2059 E-08 \\
1.8854 E-08 \\
1.0000 E+00\end{array}$ & $\begin{array}{l}3.0117 E+00 \\
7.7245 E-01 \\
7.6551 E-01 \\
7.3010 E-01 \\
1.3191 E+00 \\
1.3331 E+00 \\
1.0635 E+00 \\
7.5709 E-01 \\
7.9006 E-01 \\
8.0306 E-01 \\
7.9774 E=01 \\
1.1960 E+00 \\
1.6019 E+00 \\
1.7139 E+00 \\
1.6582 E+00 \\
2.0176 E+00 \\
2.0210 E+00 \\
2.0622 E+00 \\
1.4586 E+00 \\
1.1242 E+00 \\
1.0806 E+00 \\
1.0661 \mathrm{E}+00 \\
9.8783 \mathrm{E}-01 \\
5.1827 \mathrm{E}-01 \\
5.9007 \mathrm{E}-01\end{array}$ & $\begin{array}{l}4.6127 E-06 \\
1.8753 E-05 \\
3.7005 E-05 \\
5.3873 E-05 \\
1.6010 E-04 \\
6.3052 E-04 \\
1.0707 E-03 \\
1.1237 E-03 \\
9.2725 E-04 \\
9.0188 E-04 \\
6.8780 E-04 \\
4.9164 E-04 \\
3.2142 E-04 \\
6.4715 E-05 \\
2.0856 E-05 \\
8.4860 E-05 \\
2.0004 E-06 \\
9.2634 E-07 \\
2.4473 E-07 \\
1.1016 E-07 \\
1.4263 E-08 \\
1.7073 E-09 \\
2.4987 E-10 \\
1.9153 E-11 \\
1.0479 E-10 \\
6.7673 E-03\end{array}$ & $\begin{array}{l}5.5822 E-02 \\
1.0382 E-02 \\
8.65 C 9 E-03 \\
6.9504 E-03 \\
9.6701 E-03 \\
9.9000 E-03 \\
5.0679 E-03 \\
1.9589 E-03 \\
1.2225 E-03 \\
8.5340 E-04 \\
6.4559 E-04 \\
6.4929 E-04 \\
7.5194 E-04 \\
6.3773 E-04 \\
6.1781 E-04 \\
6.6212 E-04 \\
8.5622 E-04 \\
1.1527 E-03 \\
8.3440 E-04 \\
6.9205 E-04 \\
6.6927 E-04 \\
6.6251 E-04 \\
5.8910 E-04 \\
5.6680 E-04 \\
9.1953 E-04\end{array}$ \\
\hline
\end{tabular}

TABLE $8-X L$ I

ZPR-3-6F 25-GROUP ZONE FISSION TRRCTIONS

\begin{tabular}{|c|c|c|c|c|}
\hline reva & $\begin{array}{r}\text { Energy } \\
\text { 8oundory } \\
\text { ley. } \\
\end{array}$ & $\begin{array}{r}\text { Lolhorgy } \\
\text { Hudih }\end{array}$ & Core & Rerlecter \\
\hline $\begin{array}{l}1 \\
2 \\
3 \\
4 \\
5 \\
6 \\
7 \\
8 \\
9 \\
10 \\
11 \\
12 \\
13 \\
14 \\
15 \\
16 \\
17 \\
18 \\
19 \\
20 \\
21 \\
22 \\
23 \\
24 \\
25\end{array}$ & 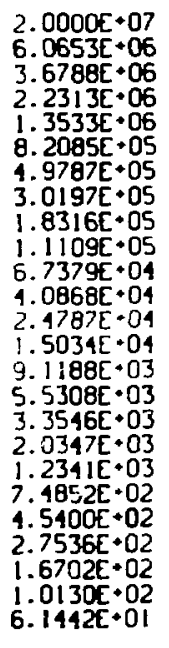 & $\begin{array}{l}\text { 1. } 1931 E+00 \\
5.0000 E-01 \\
5.0000 E-01 \\
5.0000 E-01 \\
5.0000 E-01 \\
5.0000 E-01 \\
5.0000 E-0 ! \\
5.0000 E-0 ! \\
5.0000 E-0 ! \\
5.0000 E-0 ! \\
5.0000 E-01 \\
5.0000 E-0 ! \\
5.0000 E-0 ! \\
5.0000 E-0 ! \\
5.0000 E-0 ! \\
5.0000 E-01 \\
5.0000 E-0 ! \\
5.0000 E-0 ! \\
5.0000 E-01 \\
5.0000 E-01 \\
5.0000 E-01 \\
5.0000 E-01 \\
5.0000 E-01 \\
5.0000 E-01 \\
1.7500 E+00\end{array}$ & $\begin{array}{l}2.5932 E-02 \\
1.1341 E-01 \\
2.1241 E-01 \\
2.2915 E-01 \\
1.7625 E-01 \\
1.1253 E-01 \\
6.3964 E-02 \\
3.3848 E-02 \\
1.7249 E-02 \\
8.5085 E-03 \\
4.0691 E-03 \\
1.9254 E-03 \\
9.0867 E-04 \\
1.2833 E-04 \\
2.0183 E-04 \\
9.5103 E-05 \\
4.4824 E-05 \\
2.1132 E-05 \\
9.9658 E-06 \\
4.7012 E-06 \\
2.2182 E-06 \\
1.0469 E-06 \\
4.9414 E-07 \\
2.3328 E-07 \\
2.0870 E-07 \\
1.0000 E-00\end{array}$ & 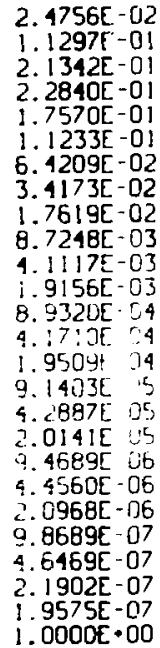 \\
\hline
\end{tabular}


TPBLE B-XL」I

ZPR-3-II 70-GROUP IRANSPORT THEORY SPECTRA

\begin{tabular}{|c|c|c|c|c|c|c|c|}
\hline cong & $\begin{array}{r}\begin{array}{r}\text { Energy } \\
\text { Boundory } \\
(1 \times y)\end{array} \\
\end{array}$ & $\begin{array}{r}\text { Lethorgy } \\
\text { Midth }\end{array}$ & $\begin{array}{r}\text { Reoctor } \\
\text { Fiveion } \\
\text { Frocluone }\end{array}$ & $\begin{array}{r}\text { Central } \\
F l u x \\
\ln \left\langle\cos ^{2} / 2\right|\end{array}$ & $\begin{array}{r}\text { Centrol } \\
\text { Pd joint } \\
\text { lehotncel }\end{array}$ & $\begin{array}{r}E d g \circ \\
F \operatorname{lux} \\
\ln \left\langle\mathrm{cos}^{2} /{ }^{2} l\right.\end{array}$ & $\begin{array}{r}\text { Edgo } \\
\text { Ad jount } \\
\text { lchoto } / \mathrm{l} \text {. }\end{array}$ \\
\hline $\begin{array}{l}1 \\
2 \\
3 \\
1 \\
5 \\
6 \\
7 \\
8 \\
9 \\
10 \\
11 \\
12 \\
13 \\
14 \\
15 \\
10 \\
17 \\
18 \\
19 \\
20 \\
21 \\
22 \\
23 \\
24 \\
25 \\
26 \\
27 \\
28 \\
29 \\
30 \\
31 \\
32 \\
33 \\
34 \\
35 \\
36 \\
37 \\
38 \\
39 \\
40 \\
41 \\
42 \\
43 \\
44 \\
45 \\
46 \\
47 \\
48 \\
49 \\
50 \\
51 \\
52 \\
53 \\
54 \\
55 \\
56 \\
57 \\
58 \\
59 \\
60 \\
61 \\
62 \\
63 \\
64 \\
65 \\
66 \\
67 \\
69 \\
70\end{array}$ & 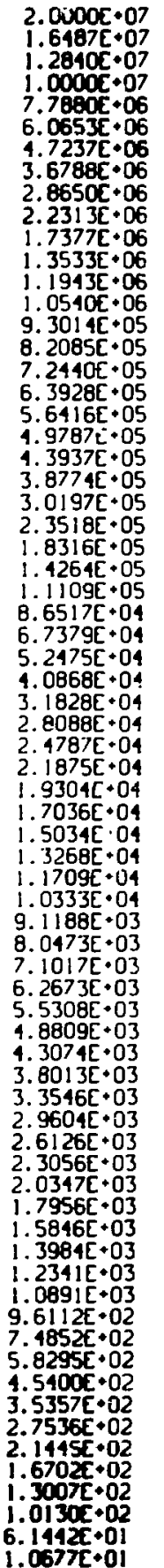 & $\begin{array}{l}1.9315 E-01 \\
2.5000 E-01 \\
2.5000 E-01 \\
2.5000 E-01 \\
2.5000 E-01 \\
2.5000 E-01 \\
2.5000 E-01 \\
2.5000 E-01 \\
2.5000 E-01 \\
2.5000 E-01 \\
2.5000 E-01 \\
1.2500 E-01 \\
1.2500 E-01 \\
1.2500 E-01 \\
1.2500 E-01 \\
1.2500 E-01 \\
1.2500 E-01 \\
1.2500 E-01 \\
1.2500 E-01 \\
1.2500 E-01 \\
1.2500 E-01 \\
2.5000 E-01 \\
2.5000 E-01 \\
2.5000 E-01 \\
2.5000 E-01 \\
2.5000 E-01 \\
2.5000 E-01 \\
2.5000 E-01 \\
2.5000 E-01 \\
2.5000 E-01 \\
2.5000 E-01 \\
1.2500 E-01 \\
1.2500 E-01 \\
1.2500 E-01 \\
1.2500 E-01 \\
1.2500 E-01 \\
1.2500 E-01 \\
1.2500 E-01 \\
1.2500 E-01 \\
1.2497 E-01 \\
1.2503 E-01 \\
1.2500 E-01 \\
2.5000 E-01 \\
2.5000 E-01 \\
1.2500 E-01 \\
1.2500 E-01 \\
1.2500 E-01 \\
1.2500 E-01 \\
1.2500 E-01 \\
1.2500 E-01 \\
1.2500 E-01 \\
1.2500 E-01 \\
1.2500 E-01 \\
1.2500 E-01 \\
1.2500 E-01 \\
1.2500 E-01 \\
1.2500 E-01 \\
1.2500 E-01 \\
1.2500 E-01 \\
1.2500 E-01 \\
1.2500 E-01 \\
2.5000 E-01 \\
200 E-01 \\
200 E-01 \\
2\end{array}$ & $\begin{array}{l}6.2756 E-06 \\
1.2092 E-04 \\
1.1213 E-03 \\
5.7556 E-03 \\
1.8594 E-02 \\
1.1885 E-02 \\
7.1382 E-02 \\
9.8143 E-02 \\
1.1450 E-01 \\
1.1789 E-01 \\
1.1036 E-01 \\
5.0119 E-02 \\
4.6208 E-02 \\
4.2014 E-02 \\
3.7814 E-02 \\
3.3753 E-02 \\
2.9728 E-02 \\
2.6168 E-02 \\
2.2855 E-02 \\
1.9806 E-02 \\
1.7065 E-02 \\
2.7160 E-02 \\
1.9731 E-02 \\
1.4198 E-02 \\
1.0161 E-02 \\
7.1821 E-03 \\
5.0509 E-03 \\
3.5129 E-03 \\
2.4186 E-03 \\
1.6615 E-03 \\
1.1405 E-03 \\
4.2803 E-04 \\
3.5450 E-04 \\
2.9360 E-04 \\
2.4315 E-04 \\
2.0135 E-04 \\
1.6674 E-04 \\
1.3808 E-04 \\
1.1434 E-04 \\
9.4669 E-05 \\
7.8437 E-C 5 \\
6.4939 E-05 \\
5.3781 E-05 \\
4.4540 E-05 \\
3.6889 E-05 \\
3.0552 E-05 \\
2.5305 E-05 \\
2.0960 E-05 \\
1.7362 E-05 \\
1.4382 E-05 \\
1.1913 E-05 \\
9.8692 E-06 \\
8.1760 E-06 \\
6.7732 E-06 \\
5.6114 E-06 \\
4.6487 E-06 \\
3.8515 E-06 \\
3.1912 E-06 \\
2.6442 E-06 \\
4.0067 E-06 \\
2.7511 E-06 \\
1.8891 E-06 \\
1.2972 E-06 \\
8.9094 E-07 \\
6.1180 E-07 \\
4.2019 E-07 \\
2.8066 E-07 \\
1.9821 E-07 \\
2.2970 E-07 \\
2.0552 E-07 \\
1.0000 E+00\end{array}$ & 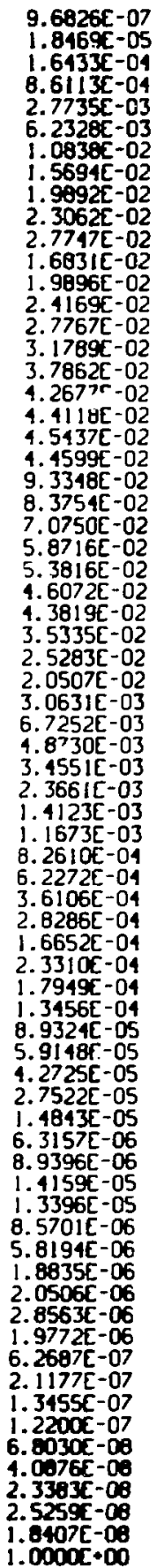 & 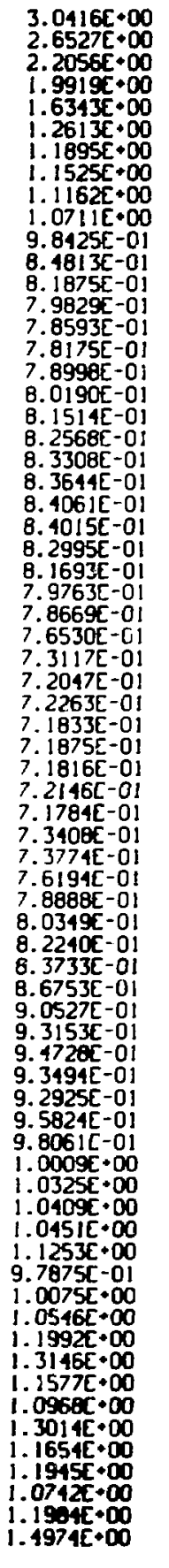 & 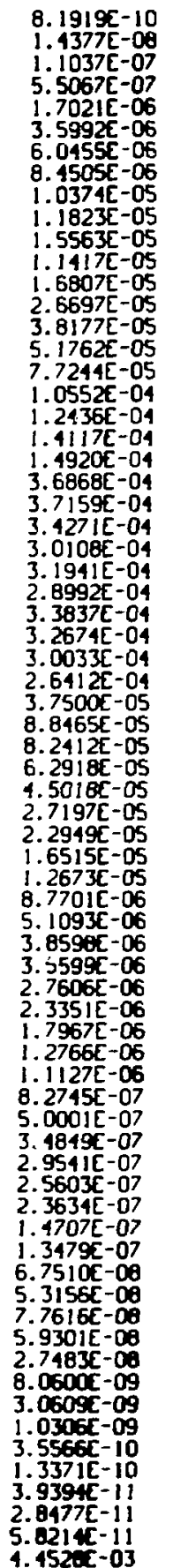 & $\begin{array}{l}2.3015 E-0 \\
1.8211 E-0 \\
1.5456 E-0 \\
1.2607 E-0 \\
9.5389 E-0 \\
8.3671 E-0 \\
7.5866 E-0 \\
7.2019 E-0 \\
6.4626 E-0 \\
5.4892 C-0 \\
3.2995 E-0 \\
3.8851 E-0 \\
4.2776 E-0 \\
4.5224 E-0 \\
4.7110 E-0 \\
1.6542 E-0 \\
4.2280 E-0 \\
3.6981 E-0 \\
3.2159 E-0 \\
2.7665 E-0 \\
2.4113 E-0 \\
1.7985 E-0 \\
1.3280 E-0 \\
1.0510 E-0 \\
8.7819 E-0 \\
7.1907 E-0 \\
6.2235 E-0 \\
5.4903 E-0 \\
4.5437 E-04 \\
3.7119 E-04 \\
3.1091 E-04 \\
3.2213 E-04 \\
3.2910 E-04 \\
3.0215 E-04\end{array}$ \\
\hline
\end{tabular}


TABLE B-XLII]

2PR-3-11 70-GROUP 2ONE FISSION FRACTIONS

\begin{tabular}{|c|c|c|c|c|}
\hline مي & $\begin{array}{r}\text { Energy } \\
\text { Boundory } \\
\text { layl }\end{array}$ & $\begin{array}{l}\text { Lethorgy } \\
\text { Nedih }\end{array}$ & Core & Anflector \\
\hline $\begin{array}{l}1 \\
2 \\
3 \\
4 \\
5 \\
6 \\
7 \\
8 \\
9 \\
10 \\
11 \\
12 \\
13 \\
14 \\
15 \\
16 \\
17 \\
18 \\
19 \\
20 \\
21 \\
22 \\
23 \\
24 \\
25 \\
26 \\
27 \\
28 \\
29 \\
30 \\
31 \\
32 \\
33 \\
34 \\
35 \\
36 \\
37 \\
38 \\
39 \\
40 \\
41 \\
42 \\
43 \\
44 \\
45 \\
46 \\
47 \\
48 \\
49 \\
50 \\
51 \\
52 \\
53 \\
54 \\
55 \\
56 \\
57 \\
58 \\
59 \\
60 \\
61 \\
62 \\
63 \\
64 \\
65 \\
66 \\
67 \\
68 \\
69 \\
70\end{array}$ & $\begin{array}{l}2.0000 E+07 \\
1.6487 E+07 \\
1.2840 E+07 \\
1.0000 E+07 \\
7.7860 E+06 \\
6.0653 E+06 \\
1.7237 E+06 \\
3.6788 E+06 \\
2.8650 E+06 \\
2.2313 E+06 \\
1.7377 E+06 \\
1.3533 E+06 \\
1.1943 E+06 \\
1.0540 E+06 \\
9.3014 E+05 \\
8.2085 E+05 \\
7.2440 E+05 \\
6.3928 E+05 \\
5.6416 E+05 \\
1.9787 E+05 \\
4.3937 E+05 \\
3.8774 E+05 \\
3.0197 E+05 \\
2.3518 E+05 \\
1.8316 E+05 \\
1.4264 E+05 \\
1.1109 E+05 \\
8.6517 E+04 \\
6.7379 E+04 \\
5.2475 E+04 \\
4.0868 E+04 \\
3.1828 E+04 \\
2.8088 E+04 \\
2.4787 E+04 \\
2.1875 E+04 \\
1.9304 E+04 \\
1.7036 E+04 \\
1.5034 E+04 \\
1.3268 E+04 \\
1.1709 E+04 \\
1.0333 E+04 \\
9.1188 E+03 \\
8.0473 E+03 \\
7.1017 E+03 \\
6.2673 E+03 \\
5.5308 E+03 \\
4.8809 E+03 \\
4.3074 E+03 \\
3.8013 E+03 \\
3.3546 E+03 \\
2.9604 E+03 \\
2.6126 E+03 \\
2.3056 E+03 \\
2.0347 E+03 \\
1.7956 E+03 \\
1.5846 E+03 \\
1.3984 E+03 \\
1.2341 E+03 \\
1.0891 E+03 \\
9.6112 E+02 \\
7.4852 E+02 \\
5.8295 E+02 \\
1.5400 E+02 \\
3.5357 E+02 \\
2.7536 E+02 \\
2.1445 E+02 \\
1.6702 E+02 \\
1.3007 E+02 \\
1.0130 E+02 \\
6.1442 E+01\end{array}$ & $\begin{array}{l}1.9315 E-01 \\
2.5000 E-01 \\
2.5000 E-01 \\
2.5000 E-01 \\
2.5000 E-01 \\
2.5000 E-01 \\
2.5000 E-01 \\
2.5000 E-01 \\
2.5000 E-01 \\
2.5000 E-01 \\
2.5000 E-01 \\
1.2500 E-01 \\
1.2500 E-01 \\
1.2500 E-01 \\
1.2500 E-01 \\
1.2500 E-01 \\
1.2500 E-01 \\
1.2500 E-01 \\
1.2500 E-01 \\
1.2500 E-01 \\
1.2500 E-01 \\
2.5000 E-01 \\
2.5000 E-01 \\
2.5000 E-01 \\
2.5000 E-01 \\
2.5000 E-01 \\
2.5000 E-01 \\
2.5000 E-01 \\
2.5000 E-01 \\
2.5000 E-01 \\
2.5000 E-01 \\
1.2500 E-01 \\
1.2500 E-01 \\
1.2500 E-01 \\
1.2500 E-01 \\
1.2500 E-01 \\
1.2500 E-01 \\
1.2500 E-01 \\
1.2000 E-01 \\
1.2497 E-01 \\
1.2503 E-01 \\
1.2500 E-01 \\
1.2500 E-01 \\
1.2500 E-01 \\
1.2500 E-01 \\
1.2500 E-01 \\
1.2500 E-01 \\
1.2500 E-01 \\
1.2500 E-01 \\
1.2500 E-01 \\
1.2500 E-01 \\
1.2500 E-01 \\
1.2500 E-01 \\
1.2500 E-01 \\
1.2500 E-01 \\
1.2500 E-01 \\
1.2500 E-01 \\
1.2500 E-01 \\
1.2500 E-01 \\
2.5000 E-01 \\
2.5000 E-01 \\
2.5000 E-01 \\
2.5000 E-01 \\
2.5000 E-01 \\
2.5000 E-01 \\
2.5000 E-01 \\
2.5000 E-01 \\
2.5000 E-01 \\
5.0000 E-01 \\
1.7500 E+00\end{array}$ & $\begin{array}{l}\text { E. 3632E-06 } \\
1.2203 E-04 \\
1.1278 E-03 \\
5.7745 E-03 \\
1.8623 E-02 \\
4.1908 E-02 \\
7.1380 E-02 \\
9.8120 E-02 \\
1.144 B E-01 \\
1.1780 E-01 \\
1.3036 E-01 \\
5.0127 E-02 \\
4.6214 E-02 \\
4.2020 E-02 \\
3.7824 E-02 \\
3.3760 E-02 \\
2.9735 E-02 \\
2.6167 E-02 \\
2.2852 E-02 \\
1.9801 E-02 \\
1.7062 E-02 \\
2.7151 E-02 \\
1.9725 E-02 \\
1.4188 E-02 \\
1.0153 E-02 \\
7.1722 E-03 \\
5.0411 E-03 \\
3.5089 E-03 \\
2.1170 E-03 \\
1.6611 E-03 \\
1.1407 E-03 \\
4.2821 E-04 \\
3.5171 E-04 \\
2.9381 E-04 \\
2.4336 E-04 \\
2.0155 E-04 \\
1.6693 E-04 \\
1.3825 E-04 \\
1.1450 E-04 \\
9.4807 E-05 \\
7.8559 E-05 \\
6.5046 E-05 \\
5.3874 E-05 \\
4.4620 E-05 \\
3.6958 E-05 \\
3.0612 E-05 \\
2.5356 E-05 \\
2.1004 E-05 \\
1.7399 E-05 \\
1.4413 E-05 \\
1.1940 E-05 \\
9.8919 E-06 \\
8.1952 E-06 \\
6.7894 E-06 \\
5.6251 E-06 \\
4.6604 E-06 \\
3.8611 E-06 \\
3.1995 E-06 \\
2.6512 E-06 \\
4.0174 E-05 \\
2.7586 E-06 \\
1.8945 E-06 \\
1.3010 E-06 \\
8.9358 E-07 \\
6.1376 E-07 \\
4.2154 E-07 \\
2.8959 E-07 \\
1.9891 E-07 \\
2.3056 E-07 \\
2.0623 E-07 \\
1.0000 E+00\end{array}$ & $\begin{array}{l}\text { 5.0861E-06 } \\
1.05 B 1 E-04 \\
1.0336 E-03 \\
5.4998 E-03 \\
1.8195 E-02 \\
1.1584 E-02 \\
7.1409 E-02 \\
9.8455 E-02 \\
1.1486 E-01 \\
1.1808 E-01 \\
1.1028 E-01 \\
5.0007 E-02 \\
4.6136 E-02 \\
1.1929 E-02 \\
3.7681 E-02 \\
3.3669 E-02 \\
2.9635 E-02 \\
2.6175 E-02 \\
2.2891 E-02 \\
1.9873 E-02 \\
1.7104 E-02 \\
2.7231 E-02 \\
1.9814 E-02 \\
1.4342 E-02 \\
1.0278 E-02 \\
7.3174 E-03 \\
5.1429 E-03 \\
3.5672 E-03 \\
2.4110 E-03 \\
1.6669 E-03 \\
1.1382 E-03 \\
4.2558 E-04 \\
3.5171 E-04 \\
2.9068 E-04 \\
2.4027 E-01 \\
1.9860 E-04 \\
1.6419 E-04 \\
1.3575 E-04 \\
1.1224 E-04 \\
9.2795 E-05 \\
7.6781 E-05 \\
6.318 B E-05 \\
5.2517 E-05 \\
4.3444 E-05 \\
3.5944 E-05 \\
2.9740 E-05 \\
2.4610 E-05 \\
2.0367 E-05 \\
1.6856 E-05 \\
1.3952 E-05 \\
1.1549 E-05 \\
9.5608 E-06 \\
7.9155 E-06 \\
6.5529 E-06 \\
5.4245 E-06 \\
4.1907 E-06 \\
3.7169 E-06 \\
3.0782 E-06 \\
2.5494 E-06 \\
3.8618 E-06 \\
2.6489 E-06 \\
1.8168 E-06 \\
1.2456 E-06 \\
8.5506 E-07 \\
5.8638 E-07 \\
4.0183 E-07 \\
2.7594 E-07 \\
1.8918 E-07 \\
2.1919 E-07 \\
1.9591 E-07 \\
1.0000 E-00\end{array}$ \\
\hline
\end{tabular}


TFBLE B-XLIV

2FR-3-11 25-GROUP TRAWSPORT THEORY SPECTRA

\begin{tabular}{|c|c|}
\hline reng & $\begin{array}{r}\text { Energy } \\
\text { Boundory } \\
\text { InY } \\
\end{array}$ \\
\hline $\begin{array}{r}1 \\
2 \\
3 \\
4 \\
5 \\
6 \\
7 \\
8 \\
9 \\
10 \\
11 \\
12 \\
13 \\
14 \\
15 \\
16 \\
17 \\
18 \\
19 \\
20 \\
21 \\
22 \\
23 \\
24 \\
25\end{array}$ & 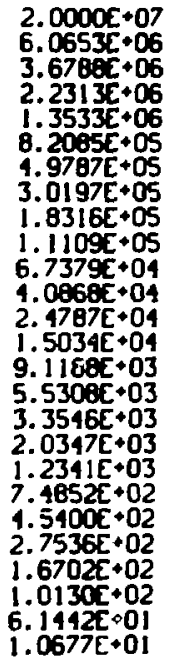 \\
\hline
\end{tabular}

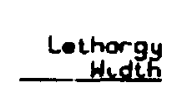

$1.1931 E+00$

5. $0000 \mathrm{E}-01$

5. $0000 \mathrm{c}-01$

5. 00002-01

5.00005-01

5. $0000 \mathrm{E}-01$

5. $0000 \mathrm{C}-01$

5.00005-01

5. $0000 \mathrm{c}-01$

5. $00005-01$

5. $00005-01$

5. c000L-0]

5. $0000 \mathrm{c}-01$

5. $0000 E-01$

$5.0000 E-01$

5. $0000 \mathrm{E}-01$

5. 0000E-01

5. $0000 \mathrm{E}-0$

5. DODOE-Ci

5. $0000 \mathrm{~L}-01$

5. $0000 \mathrm{E}-01$

5. 0000E-0!

5. $0000 \tau-01$

5. 0000L-0!

$1.7500 E+00$

\begin{tabular}{|c|}
\hline \\
\hline 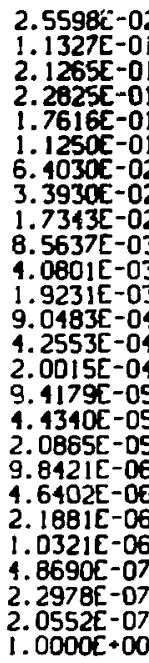 \\
\hline
\end{tabular}

$\begin{array}{r}\text { Centrol } \\ \text { FLux } \\ \text { In/cm } 2 / 11\end{array}$
$3.8183 E-03$
$1.7071 E-02$
$3.5586 E-02$
$5.0809 E-02$
$8.8663 E-02$
$1.5645 E-01$
$1.8336 E-01$
$1.5150 E-01$
$1.1253 E-01$
$8.9091 E-02$
$6.0518 E-02$
$3.0295 E-02$
$1.2107 E-02$
$2.9772 E-03$
$8.6196 E-04$
$3.2576 E-04$
$5.7620 E-05$
$4.1945 E-05$
$6.7904 E-06$
$2.6040 E-06$
$3.1632 E-07$
$1.9003 E-07$
$6.4259 E-08$
$2.5259 E-08$
$1.0407 E-08$
$1.0000 E+00$

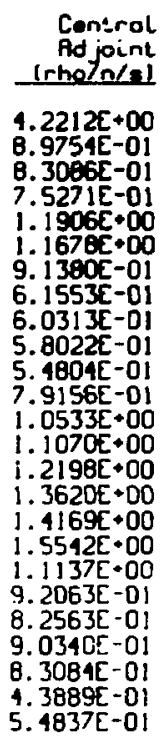

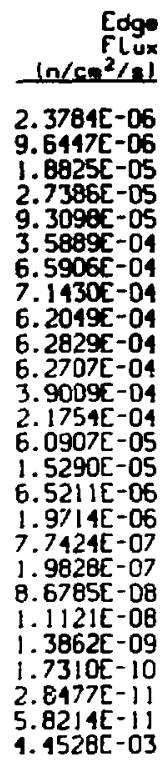

Edge
Ad jount
rholncel

3. $5692 \varepsilon-02$

$6.5576 E-03$

5. $45 \geq 3 E-03$

4.3771E-D3

6.0737E-03

6. $3325 \varepsilon-03$

3. $0739 \mathrm{E}-03$

1. $14505-03$

7.0652E-04

4. $9126 \mathrm{E}-04$

$3.6717 \mathrm{E}-04$

3.6777E-04

4. $2615 E-04$

3. $7776 E-04$

3.7554E-04

4. $0634 E-04$

5. $3002 E-04$

7. 3548E-04

5. 38] IE-04

$4.4397 E-04$

4. $0995 E-04$

4. 3868r-04

3. $9188 \mathrm{r}-04$

$3.7227 E-04$

6. $1736 E-04$

TRaLE B-XLV

ZPR-3-1i 25-GROUP ZOAE FISSION FRACTIONS

\begin{tabular}{|c|c|c|c|c|}
\hline rong & $\begin{array}{r}\text { Energy } \\
\text { Boundory } \\
\text { levI } \\
\end{array}$ & $\begin{array}{r}\text { Lechorgy } \\
\text { Hidik }\end{array}$ & Core & Reflector \\
\hline $\begin{array}{l}1 \\
2 \\
3 \\
4 \\
5 \\
6 \\
7 \\
8 \\
9 \\
10 \\
11 \\
12 \\
13 \\
14 \\
15 \\
16 \\
17 \\
18 \\
19 \\
20 \\
21 \\
22 \\
23 \\
24 \\
25\end{array}$ & 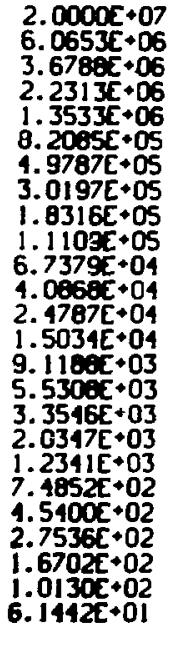 & $\begin{array}{l}1.1931 E+00 \\
5.0000 E-01 \\
5.0000 E-01 \\
5.0000 E-01 \\
5.0000 E-01 \\
5.0000 E-01 \\
5.0000 E-01 \\
5.0000 E-01 \\
5.0000 E-01 \\
5.0000 E-01 \\
5.0000 E-01 \\
5.0000 E-01 \\
5.0000 E-01 \\
5.0000 E-01 \\
5.0000 E-01 \\
5.0000 E-01 \\
5.0000 E-01 \\
5.0000 E-01 \\
5.0000 E-01 \\
5.0000 E-01 \\
5.0000 E-01 \\
5.0000 E-01 \\
5.0000 E-01 \\
5.0000 E-01 \\
1.7500 E+00\end{array}$ & 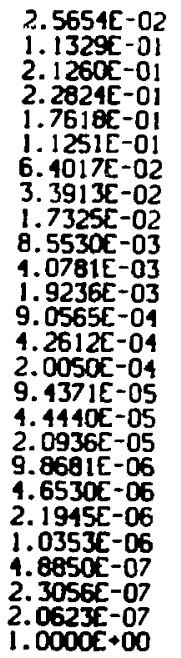 & $\begin{array}{l}2.4839 E-02 \\
1.1299 E-01 \\
2.1331 E-01 \\
2.2836 E-01 \\
1.7575 E-01 \\
1.1237 E-01 \\
6.4200 E-02 \\
3.4157 E-02 \\
1.7596 E-02 \\
8.7101 \mathrm{E}-03 \\
4.1079 \mathrm{E}-03 \\
1.9155 \mathrm{E}-03 \\
8.9374 \mathrm{E}-04 \\
4.1756 \mathrm{E}-04 \\
1.9539 E-04 \\
9.1572 E-05 \\
4.2977 \mathrm{E}-05 \\
2.0185 \mathrm{E}-05 \\
9.4894 \mathrm{E}-06 \\
4.4657 \mathrm{E}-06 \\
2.1006 \mathrm{E}-06 \\
9.8821 \mathrm{E}-07 \\
4.6512 \mathrm{E}-07 \\
2.1919 \mathrm{E}-07 \\
1.9591 \mathrm{E}-07 \\
1.0000 \mathrm{E}+00\end{array}$ \\
\hline
\end{tabular}


TABLE B-XLVI

2PR-3-12 70-GROUP TRPWSPORT THEORY SPECTRA

\begin{tabular}{|c|c|c|c|c|c|c|c|}
\hline -ang & $\begin{array}{r}\text { Enorgy } \\
\text { Boundory } \\
\text { (ny) }\end{array}$ & $\begin{array}{r}\text { Lothorgy } \\
\text { Hidth }\end{array}$ & $\begin{array}{r}\text { Reoctor } \\
\text { Fiesion } \\
\text { Fractionn }\end{array}$ & $\begin{array}{r}\text { Contral } \\
\text { Flux } \\
\ln / \cos ^{2} / \operatorname{lol}^{2}\end{array}$ & $\begin{array}{r}\text { Centrol } \\
\text { Ad joint } \\
\text { lrholntal }\end{array}$ & $\begin{array}{r}\text { Edge } \\
F_{\text {Lux }} \\
\ln \left\langle\mathrm{cos}^{2} / \mathrm{l}\right)\end{array}$ & $\begin{array}{r}\text { Edge } \\
\text { Ad jount } \\
\text { Inholn } / n \text { l }\end{array}$ \\
\hline $\begin{array}{l}1 \\
2 \\
3 \\
4 \\
5 \\
6 \\
7 \\
8 \\
9 \\
10 \\
11 \\
12 \\
13 \\
14 \\
15 \\
16 \\
17 \\
18 \\
19 \\
20 \\
21 \\
22 \\
23 \\
24 \\
25 \\
26 \\
27 \\
28 \\
29 \\
30 \\
31 \\
32 \\
33 \\
34 \\
35 \\
36 \\
37 \\
38 \\
39 \\
40 \\
41 \\
42 \\
43 \\
44 \\
45 \\
46 \\
47 \\
43 \\
19 \\
50 \\
51 \\
52 \\
53 \\
54 \\
55 \\
56 \\
57 \\
58 \\
59 \\
50 \\
61 \\
52 \\
53 \\
64 \\
65 \\
65 \\
67 \\
58 \\
59 \\
70\end{array}$ & $\begin{array}{l}2.0000 E+07 \\
1.6487 E+07 \\
1.2840 E+07 \\
1.0000 E+07 \\
7.7880 E+06 \\
6.0653 E+06 \\
4.7237 E+06 \\
3.6788 E+06 \\
2.8650 E+06 \\
2.2313 E+06 \\
1.7377 E+06 \\
1.3533 E+06 \\
1.1943 E+06 \\
1.0540 E+06 \\
9.3014 E+05 \\
8.2085 E+05 \\
7.2440 E+05 \\
6.3928 E+05 \\
5.6416 E+05 \\
4.9787 E+05 \\
1.3937 E+05 \\
3.8774 E+05 \\
3.0197 E+05 \\
2.3518 E+05 \\
1.8316 E+05 \\
1.4261 E+05 \\
1.1109 E+05 \\
8.6517 E+04 \\
6.7379 E+04 \\
5.2475 E+04 \\
4.0368 E+04 \\
3.1828 E+04 \\
2.8088 E+04 \\
2.4787 E+04 \\
2.1875 E+04 \\
1.9304 E+01 \\
1.7036 E+04 \\
1.5034 E+04 \\
1.3268 E+04 \\
1.1709 E+04 \\
1.0333 E+04 \\
9.1188 E+03 \\
8.0473 E+03 \\
7.1017 E+03 \\
6.2673 E+03 \\
5.5308 E+03 \\
4.8809 E+03 \\
4.3074 E+03 \\
3.8013 E+03 \\
3.3546 E+03 \\
2.9604 E+03 \\
2.6126 E+03 \\
2.3056 E+03 \\
2.0347 E+03 \\
1.7956 E+03 \\
1.5846 E+03 \\
1.3984 E+03 \\
1.2341 E+03 \\
1.0891 E+03 \\
9.6112 E+02 \\
7.4852 E+02 \\
5.8295 E+02 \\
4.5400 E+02 \\
3.5357 E+02 \\
2.7536 E+02 \\
2.1445 E+02 \\
1.6702 E+02 \\
1.3007 E+02 \\
1.0130 E+02 \\
6.1442 E+01 \\
1.5775+01\end{array}$ & $\begin{array}{l}1.9315 E-01 \\
2.5000 E-01 \\
2.5000 E-01 \\
2.5000 E-01 \\
2.5000 E-01 \\
2.5000 E-01 \\
2.5000 E-01 \\
2.5000 E-01 \\
2.5000 E-01 \\
2.5000 E-01 \\
2.5000 E-01 \\
1.2500 E-01 \\
1.2500 E-01 \\
1.2500 E-01 \\
1.2500 E-01 \\
1.2500 E-01 \\
1.2500 E-01 \\
1.2500 E-01 \\
1.2500 E-01 \\
1.2500 E-01 \\
1.2500 E-01 \\
2.5000 E-01 \\
2.5000 E-01 \\
2.5000 E-01 \\
2.5000 E-01 \\
2.5000 E-01 \\
2.5000 E-01 \\
2.5000 E-01 \\
2.5000 E-01 \\
2.5000 E-01 \\
2.5000 E-01 \\
1.2500 E-01 \\
1.2500 E-01 \\
1.2500 E-01 \\
1.2500 E-01 \\
1.2500 E-01 \\
1.2500 E-01 \\
1.2500 E-01 \\
1.2500 E-01 \\
1.2497 E-01 \\
1.2503 E-01 \\
1.2500 E-01 \\
1.2500 E-01 \\
1.2500 E-01 \\
1.2500 E-01 \\
1.2500 E-01 \\
1.2500 E-01 \\
1.2500 E-01 \\
1.2500 E-01 \\
1.2500 E-01 \\
1.2500 E-01 \\
1.2500 E-01 \\
1.2500 E-01 \\
1.2500 E-01 \\
1.2500 E-01 \\
1.2500 E-01 \\
1.2500 E-01 \\
1.2500 E-01 \\
1.2500 E-01 \\
2.5000 E-01 \\
2.5000 E-01 \\
2.5000 E-01 \\
2.5000 E-01 \\
2.5000 E-01 \\
2.5000 E-01 \\
2.5000 E-01 \\
2.5000 E-01 \\
5.000 E-01 \\
1.7500 E-01 \\
1.000\end{array}$ & $\begin{array}{l}6.3513 E-06 \\
1.2193 E-04 \\
1.1272 E-03 \\
5.7725 E-03 \\
1.8619 E-02 \\
4.1901 E-02 \\
7.1374 E-02 \\
9.8117 E-02 \\
1.1418 E-01 \\
1.1788 E-01 \\
1.1036 E-01 \\
5.0123 E-02 \\
4.6214 E-02 \\
4.2020 E-02 \\
3.7825 E-02 \\
3.3760 E-02 \\
2.9736 E-02 \\
2.6168 E-02 \\
2.2853 E-02 \\
1.9802 E-02 \\
1.7063 E-02 \\
2.7157 E-02 \\
1.9727 E-02 \\
1.418 G E-02 \\
1.0151 E-02 \\
7.1728 E-03 \\
5.0445 E-03 \\
3.5093 E-03 \\
2.4173 E-03 \\
1.6614 E-03 \\
1.1409 E-03 \\
4.2830 E-04 \\
3.5479 E-04 \\
2.9388 E-04 \\
2.4342 E-04 \\
2.0160 E-04 \\
1.6697 E-04 \\
1.3829 E-04 \\
1.1453 E-04 \\
9.4833 E-05 \\
7.8581 E-05 \\
6.5065 E-05 \\
5.3889 E-05 \\
4.4633 E-05 \\
3.6969 E-05 \\
3.0621 E-05 \\
2.5364 E-05 \\
2.1011 E-05 \\
1.7404 E-05 \\
1.4418 E-05 \\
1.1944 E-05 \\
9.8951 E-06 \\
8.1979 E-06 \\
6.7917 E-06 \\
5.6269 E-06 \\
4.6619 E-06 \\
3.8626 E-06 \\
3.2005 E-06 \\
2.6520 E-06 \\
4.0186 E-06 \\
2.7595 E-06 \\
1.8950 E-06 \\
1.3013 E-06 \\
8.9383 E-07 \\
6.1391 E-07 \\
4.2162 E-07 \\
2.8965 E-07 \\
1.9095 E-07 \\
2.3060 E-07 \\
2.0627 E-07 \\
1.0000 E+00\end{array}$ & 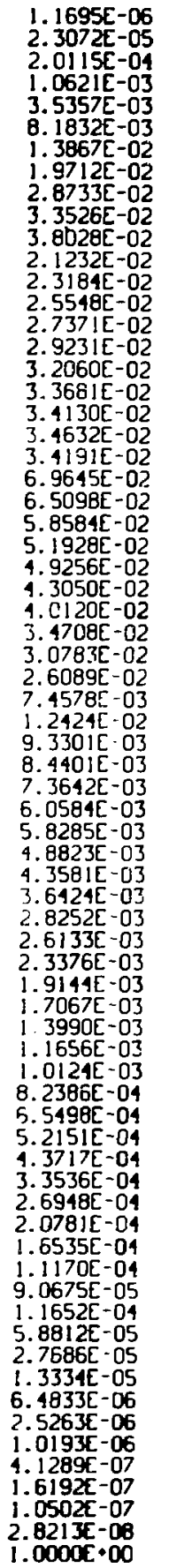 & $\begin{array}{l}2.3952 E+00 \\
2.1623 E+00 \\
1.8093 E+00 \\
1.6516 E+00 \\
1.4199 E+00 \\
1.1491 E+00 \\
1.1268 E+00 \\
1.1081 E+00 \\
1.0757 E+00 \\
1.0344 E+00 \\
3.6030 E-01 \\
8.7860 E-01 \\
8.6327 E-01 \\
8.5495 E-01 \\
8.5356 E-01 \\
8.5915 E-01 \\
9.6870 E-01 \\
8.8137 E-01 \\
8.9564 E-01 \\
9.0744 E-01 \\
9.1727 E-01 \\
9.2568 E-01 \\
9.3855 E-01 \\
9.4 B 79 E-01 \\
9.5179 E-01 \\
9.5109 E-01 \\
9.4981 E-01 \\
9.4770 E-0 ! \\
9.1326 E-01 \\
9.4059 E-01 \\
9.4828 E-01 \\
9.6215 E-01 \\
9.6159 E-01 \\
9.5738 E-01 \\
9.7612 E-01 \\
9.9029 E-01 \\
9.9762 E-01 \\
1.0134 E+00 \\
1.0232 E+00 \\
1.0409 E+00 \\
1.0600 E+00 \\
1.0755 E+00 \\
1.0928 E+00 \\
1.1103 E+00 \\
1.1282 E+00 \\
1.1519 E+00 \\
1.1621 E+00 \\
1.1750 E+00 \\
1.1740 E+00 \\
1.1728 E+00 \\
1.1881 E+00 \\
1.1980 E+00 \\
1.1992 E+00 \\
1.2274 E+00 \\
1.2386 E+00 \\
1.2324 E+00 \\
1.2749 E+00 \\
1.2240 E+00 \\
1.2409 E+00 \\
1.2855 E+00 \\
1.3839 E+00 \\
1.1413 E+00 \\
1.3667 E+00 \\
1.3809 E+00 \\
1.4050 E+00 \\
1.2966 E+00 \\
1.2911 E+00 \\
1.2469 E+00 \\
1.3287 E+00 \\
1.5668 E+00\end{array}$ & 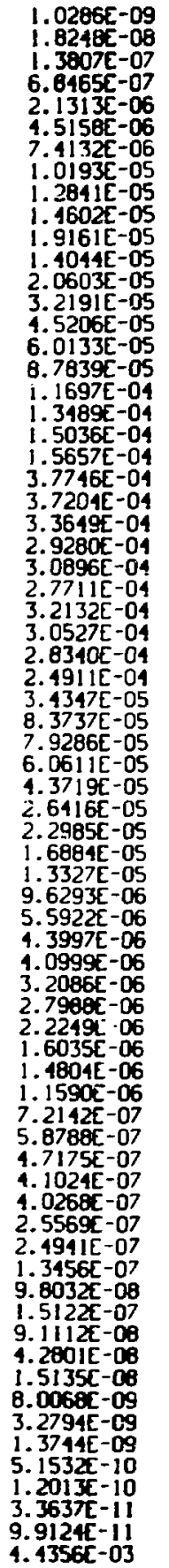 & 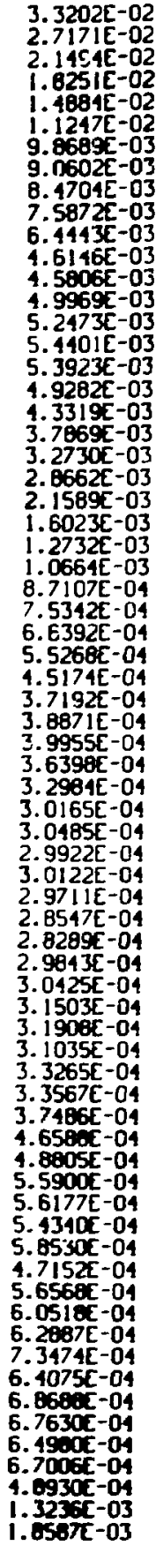 \\
\hline
\end{tabular}


TABLE B-XLVII

ZPR-3-12 70-GROUP ZONE FISSION FRACTIONS

\begin{tabular}{|c|c|c|c|c|}
\hline Soup & $\begin{array}{r}\text { Energy } \\
\text { Boundory } \\
\text { (ev) }\end{array}$ & $\begin{array}{l}\text { Lethorgy } \\
\text { Widith }\end{array}$ & Core & Reflector \\
\hline 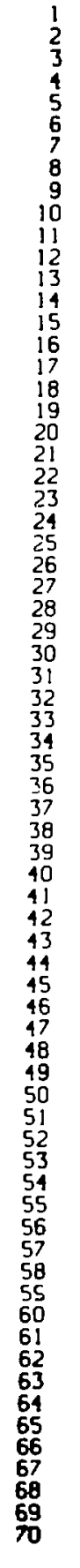 & $\begin{array}{l}2.0000 E+07 \\
1.6487 E+07 \\
1.2840 E+07 \\
1.0000 E+07 \\
7.7880 E+06 \\
6.0653 E+06 \\
4.7237 E+06 \\
3.6788 E+06 \\
2.8650 E+06 \\
2.2313 E+06 \\
1.7377 E+06 \\
1.3533 E+06 \\
1.1943 E+06 \\
1.0540 E+06 \\
9.3014 E+05 \\
8.2085 E+05 \\
7.2440 E+05 \\
6.3928 E+05 \\
5.6416 E+05 \\
1.9787 E+05 \\
4.3937 E+05 \\
3.8774 E+05 \\
3.0197 E+05 \\
2.3518 E+05 \\
1.8316 E+05 \\
1.1261 E+05 \\
1.1109 E+05 \\
8.6517 E+04 \\
6.7379 E+04 \\
5.2475 E+04 \\
4.0868 E+04 \\
3.1828 E+04 \\
2.8088 E+04 \\
2.4787 E+04 \\
2.1875 E+04 \\
1.9304 E+04 \\
1.7036 E+04 \\
1.5034 E+04 \\
1.3268 E+04 \\
1.17 C 9 E+04 \\
1.0333 E+04 \\
9.1188 E+03 \\
8.0473 E+03 \\
7.1017 E+03 \\
6.2673 E+03 \\
5.5308 E+03 \\
4.8809 E+03 \\
4.3074 E+03 \\
3.8013 E+03 \\
3.3546 E+03 \\
2.9604 E+03 \\
2.6126 E+03 \\
2.3056 E+03 \\
2.0317 E+03 \\
1.7956 E+03 \\
1.5846 E+03 \\
1.3984 E+03 \\
1.2341 E+03 \\
1.0891 E \cdot 03 \\
9.6112 E+02 \\
7.4852 E+02 \\
5.8295 E+02 \\
4.5400 E+02 \\
3.5357 E+02 \\
2.7536 E+02 \\
2.1445 E+02 \\
1.6702 E+02 \\
1.3007 E+02 \\
1.0130 E+02 \\
6.1442 E+01\end{array}$ & $\begin{array}{l}1.9315 E-01 \\
2.5000 E-01 \\
2.5000 E-01 \\
2.5000 E-01 \\
2.5000 E-01 \\
2.5000 E-01 \\
2.5000 E-01 \\
2.5000 E-01 \\
2.5000 E-01 \\
2.5000 E-01 \\
2.5000 E-01 \\
1.2500 E-01 \\
1.2500 E-01 \\
1.2500 E-01 \\
1.2500 E-01 \\
1.2500 E-01 \\
1.2500 E-01 \\
1.2500 E-01 \\
1.2500 E-01 \\
1.2500 E-01 \\
1.2500 E-01 \\
2.5000 E-01 \\
2.5000 E-01 \\
2.5000 E-01 \\
2.5000 E-01 \\
2.5000 E-01 \\
2.5000 E-01 \\
2.5000 E-01 \\
2.5000 E-01 \\
2.5000 E-01 \\
2.5000 E-01 \\
1.2500 E-01 \\
1.2500 E-01 \\
1.2500 E-01 \\
1.2500 E-01 \\
1.2500 E-01 \\
1.2500 E-01 \\
1.2500 E-01 \\
1.2500 E-01 \\
1.2497 E-01 \\
1.2503 E-01 \\
1.2500 E-01 \\
1.2500 E-01 \\
1.2500 E-01 \\
1.2500 E-01 \\
1.2500 E-01 \\
1.2500 E-01 \\
1.2500 E-01 \\
1.2500 E-01 \\
1.2500 E-01 \\
1.2500 E-01 \\
1.2500 E-01 \\
1.2500 E-01 \\
1.2500 E-01 \\
1.2500 E-01 \\
1.2500 E-01 \\
1.2500 E-01 \\
1.2500 E-01 \\
1.2500 E-01 \\
2.5000 E-01 \\
2.5000 E-01 \\
2.5000 E-01 \\
2.5000 E-01 \\
2.5000 E-01 \\
2.5000 E-01 \\
2.5000 E-01 \\
2.5000 E-01 \\
2.5000 E-01 \\
5.0000 E-01 \\
1.7500 E-00 \\
2.01\end{array}$ & $\begin{array}{l}6.5120 E-06 \\
1.2398 E-04 \\
1.1391 E-03 \\
5.8073 E-03 \\
1.8673 E-02 \\
4.1942 E-02 \\
7.1369 E-02 \\
9.8074 E-02 \\
1.1443 E-01 \\
1.1786 E-01 \\
1.1037 E-01 \\
5.0144 E-02 \\
4.6224 E-02 \\
4.2032 E-02 \\
3.7843 E-02 \\
3.3772 E-02 \\
2.9749 E-02 \\
2.6167 E-02 \\
2.2848 E-02 \\
1.9793 E-02 \\
1.7058 E-02 \\
2.7147 E-02 \\
1.9716 E-02 \\
1.4169 E-02 \\
1.0137 E-02 \\
7.1543 E-03 \\
5.0319 E-03 \\
3.5019 E-03 \\
2.4143 E-03 \\
1.66 C 7 E-03 \\
1.1413 E-03 \\
4.2863 E-04 \\
3.5517 E-04 \\
2.9428 E-04 \\
2.4381 E-04 \\
2.0198 E-04 \\
1.6732 E-04 \\
1.3861 E-04 \\
1.1482 E-04 \\
9.5091 E-05 \\
7.8809 E-05 \\
6.5264 E-05 \\
5.40 E 3 E-05 \\
4.4784 E-05 \\
3.7099 E-05 \\
3.0733 E-05 \\
2.5459 E-05 \\
2.1092 E-05 \\
1.7474 E-05 \\
1.4477 E-05 \\
1.1994 E-05 \\
9.9374 E-06 \\
8.2336 E-06 \\
6.8219 E-06 \\
5.6525 E-06 \\
4.6835 E-06 \\
3.8809 E-06 \\
3.2159 E-06 \\
2.6649 E-06 \\
4.0384 E-06 \\
2.7734 E-06 \\
1.9048 E-06 \\
1.3083 E-06 \\
8.9865 E-07 \\
6.1731 E-07 \\
1.2405 E-07 \\
2.9133 E-07 \\
2.0011 E-07 \\
2.3200 E-07 \\
2.0753 E-07 \\
1.0000 E-00\end{array}$ & $\begin{array}{l}5.0269 E-06 \\
1.0501 E-04 \\
1.0287 E-03 \\
5.4851 E-03 \\
1.8172 E-02 \\
4.1565 E-02 \\
7.140 B E-02 \\
9.8471 E-02 \\
1.1487 E-01 \\
1.1809 E-01 \\
1.1028 E-01 \\
5.0000 E-02 \\
4.6131 E-02 \\
4.1924 E-02 \\
3.7674 E-02 \\
3.3664 E-02 \\
2.9630 E-02 \\
2.6176 E-02 \\
2.2894 E-02 \\
1.9877 E-02 \\
1.7108 E-02 \\
2.7237 E-02 \\
1.9820 E-02 \\
1.4351 E-02 \\
1.0286 E-02 \\
7.3253 E-03 \\
5.1483 E-03 \\
3.5705 E-03 \\
2.4425 E-03 \\
1.6674 E-03 \\
1.1382 E-03 \\
4.2551 E-04 \\
3.5161 E-04 \\
2.9057 E-04 \\
2.4015 E-04 \\
1.9849 E-04 \\
1.6408 E-04 \\
1.3564 E-04 \\
1.1215 E-04 \\
9.2711 E-05 \\
7.6707 E-05 \\
6.3422 E-05 \\
5.2459 E-05 \\
4.3194 E-05 \\
3.5900 E-05 \\
2.9703 E-05 \\
2.4577 E-05 \\
2.0339 E-05 \\
1.6832 E-05 \\
1.3932 E-05 \\
1.1532 E-05 \\
9.5463 E-06 \\
7.9031 E-06 \\
6.5426 E-06 \\
5.4162 E-06 \\
4.1839 E-06 \\
3.7116 E-06 \\
3.0737 E-0 E \\
2.5457 E-06 \\
3.8558 E-06 \\
2.6449 E-06 \\
1.8142 E-06 \\
1.2441 E-06 \\
8.5401 E-07 \\
5.8580 E-07 \\
4.0160 E-07 \\
2.7578 E-07 \\
1.8913 E-07 \\
2.1912 E-07 \\
1.9585 E-07 \\
1.0000 E+00\end{array}$ \\
\hline
\end{tabular}


TABLE $B-X L Y I I I$

ZPR-3-12 25-GROUP TRANSPORT THEORY SPECTRA

$\begin{array}{cr}\text { Group } & \begin{array}{r}\text { Enorgy } \\ \text { Boundory } \\ \text { lovl }\end{array} \\ 1 & \begin{array}{r}2.0000 E+07 \\ 2\end{array} \\ 3 & 3.0653 E+06 \\ 4 & 2.2313 E+06 \\ 5 & 1.3533 E+06 \\ 6 & 0.2085 E+05 \\ 7 & 4.9787 E+05 \\ 8 & 3.0197 E+05 \\ 9 & 1.8316 E+05 \\ 10 & 1.1109 E \cdot 05 \\ 11 & 6.7379 E+04 \\ 12 & 4.0868 E+04 \\ 13 & 2.4787 E+04 \\ 14 & 1.5034 E+04 \\ 15 & 9.1188 E+03 \\ 16 & 5.5308 E+03 \\ 17 & 3.3546 E+03 \\ 18 & 2.0347 E+03 \\ 19 & 1.2341 E+03 \\ 20 & 7.4852 E+02 \\ 21 & 4.5400 E+02 \\ 12 & 2.7536 E+02 \\ 23 & 1.6702 E+02 \\ 24 & 1.0130 E+02 \\ 25 & 6.1412 E+01 \\ & 1.0677 E+01\end{array}$

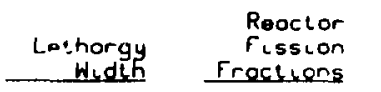

1. $1931 E+00$

5. OOCOE-01

S. $00005-01$

5. $0000 \mathrm{E}-01$

5. OOUOE-0I

5. $0000 E-01$

5. $0000 E-01$

5. $0000 \mathrm{E}-01$

$5.0000 \mathrm{E}-01$

5. $0000[-01$

5. $0000 E-01$

5. OONOE-01

5. 0000E-01

5. $0000 E-01$

5. 0000E-01

5. $0000 \mathrm{E}-01$

5. $0000 \mathrm{E}-01$

5. 0000E-0I

5. O000E-01

5. 0000E - 01

5. 0000E-01

5. 0000द-0?

5. 0000E-0

5. $0000 E-01$

1. $7500 \mathrm{E}+00$
Fractions

2. 5647E-02

1. $1327 E-01$

2. !260E-0!

2. 2824E-0!

1. $7619 E-01$

1. . $252[-01$

3. $3916 E-02$

1. $7326 E-02$

$8.5538 E-03$

4. $0787 E-03$

1. $9240 E-03$

9. $0587 E-04$

4. $2623 E-04$

2. $0056 E-04$

9. $4400 E-05$

$4.4455 E-05$

2.0343E-05

Q. B7!1E-06

4. 6544E-06

2. $1951 E-36$

1. $0355 E-06$

4.8860 - 0 ?

2. $3060 E-07$

2. $0627 E-07$
6. $4022 \mathrm{E}-02$

\begin{tabular}{|c|c|}
\hline $\begin{array}{r}\text { Control } \\
r(\operatorname{lux} \\
\left(n / \mathrm{cm}^{2} / \mathrm{s}\right) \\
\end{array}$ & $\begin{array}{r}\text { Centrol } \\
\text { Ad joint } \\
\text { |rhotnis| } \\
\end{array}$ \\
\hline $\begin{array}{l}4.8232 E-03 \\
2.2050 E-02 \\
4.8445 E-02 \\
7.1554 E-02 \\
9.7336 E-02 \\
1.2910 E-01 \\
1.3847 E-01 \\
1.2368 E-01 \\
3.0118 E-01 \\
8.3171 E-02 \\
6.5491 E-02 \\
4.5971 \mathrm{E}-02 \\
3.1193 \mathrm{E}-02 \\
1.8711 \mathrm{E}-02 \\
9.6505 E-03 \\
5.2838 E-03 \\
2.4375 E-03 \\
9.7799 E-04 \\
3.1889 E-04 \\
8.5498 E-05 \\
1.9818 E-05 \\
3.5456 E-06 \\
5.7481 E-07 \\
1.0502 E-07 \\
2.3213 E-0 B \\
1.0000 E-00\end{array}$ & $\begin{array}{l}3.4547 E+00 \\
8.3305 E-01 \\
7.9935 E-01 \\
7.3011 E-01 \\
1.2629 E+00 \\
1.2829 E+00 \\
1.0067 E+00 \\
6.9082 E-01 \\
6.9651 E-0 ! \\
6.945 E-0 ! \\
6.8955 E-01 \\
1.0512 E-00 \\
1.4391 E \cdot 00 \\
1.5145 E \cdot 00 \\
1.6130 E+00 \\
1.7068 E+00 \\
1.7416 E-00 \\
1.8204 E \cdot 00 \\
1.3727 E \cdot 00 \\
1.0341 E \cdot 00 \\
1.0130 E \cdot 00 \\
9.8886 E-0 ! \\
9.2899 E-01 \\
4.8635 E-01 \\
5.7350 E-0 !\end{array}$ \\
\hline
\end{tabular}

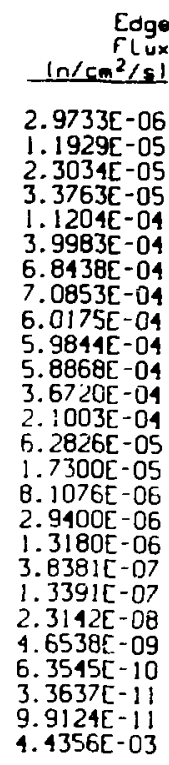

Edge
Pd jolnt
- reneinis

4. 2094E-02

$7.7291 E-03$

6. $\because$ : $E B E-\cap 3$

5. $1359 E-03$

7. $1154 \mathrm{E}-03$

7. $3545 E-03$

3. $6332 \mathrm{E}-03$

1. $3767 \mathrm{C}-0$ ?

B. $5634 \mathrm{E}-04$

5. $9461 E-04$

$4.45315-04$

4. $4376 E-04$

5. $1062 \mathrm{E}-04$

4. $4011[-04$

4. 2864 E- 04

4.6746E-04

6. 0924E-04

8.2337E-04

$6.0116 E-04$

+. $9912 \mathrm{E}-04$

4. $8595 E-04$

$4.8539 \varepsilon-04$

4. $2436 \mathrm{E}-04$

4. 8447E-04

6. 803AE-04

TABLE $B-X L] X$

ZPR-3-12 25-GROUP ZONE FISSION FRACTIONS

\begin{tabular}{|c|c|c|c|c|}
\hline irowa & $\begin{array}{r}\text { Energy } \\
\text { Boundory } \\
(0 v)^{2} \\
\end{array}$ & $\begin{array}{l}\text { Lethorgy } \\
\text { Hidth }\end{array}$ & Core & Rerlecter \\
\hline $\begin{array}{r}1 \\
2 \\
3 \\
4 \\
5 \\
6 \\
7 \\
8 \\
9 \\
10 \\
11 \\
12 \\
13 \\
14 \\
15 \\
16 \\
17 \\
10 \\
19 \\
20 \\
21 \\
22 \\
23 \\
24 \\
25\end{array}$ & 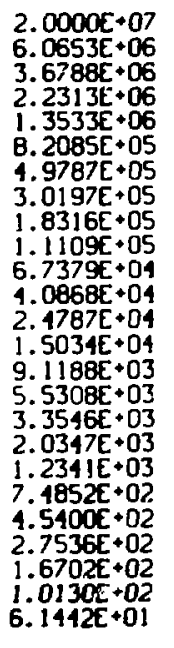 & $\begin{array}{l}1.1931 E+00 \\
5.0000 E-01 \\
5.0000 E-01 \\
5.0000 E-01 \\
5.0000 E-01 \\
5.0000 E-01 \\
5.0000 E-01 \\
5.0000 E-01 \\
5.0000 E-01 \\
5.0000 E-01 \\
5.0000 E-01 \\
5.0000 E-01 \\
5.0000 E-01 \\
5.0000 E-01 \\
5.0000 E-01 \\
5.0000 E-01 \\
5.0000 E-01 \\
5.0000 E-01 \\
5.0000 E-01 \\
5.0000 E-01 \\
5.0000 E-01 \\
5.0000 E-01 \\
5.0000 E-01 \\
5.0000 E-01 \\
1.7500 E+00\end{array}$ & $\begin{array}{l}2.5750 E-02 \\
1.1331 E-01 \\
2.1251 E-01 \\
2.2823 E-01 \\
1.7624 E-01 \\
1.1254 E-01 \\
6.3997 E-02 \\
3.3885 E-02 \\
1.7292 E-02 \\
8.5338 E-03 \\
1.0749 E-03 \\
1.9251 E-03 \\
9.0740 E-04 \\
4.2733 E-04 \\
2.0121 E-04 \\
9.4758 E-05 \\
1.4642 E-05 \\
2.1039 E-05 \\
9.9191 E-06 \\
4.6782 E-06 \\
2.2069 E-06 \\
1.0414 E-06 \\
4.9147 E-07 \\
2.3200 E-07 \\
2.0753 E-07 \\
1.0000 E+00\end{array}$ & $\begin{array}{l}2.4796 E-02 \\
1.1297 E-01 \\
2.1334 E-01 \\
2.2837 E-01 \\
1.7573 E-01 \\
1.1236 E-01 \\
6.4221 E-02 \\
3.4172 E-02 \\
1.7611 E-02 \\
8.7187 \mathrm{E}-03 \\
4.1099 \mathrm{E}-03 \\
1.9153 \mathrm{E}-03 \\
8.9329 \mathrm{E}-04 \\
4.1721 \mathrm{E}-04 \\
1.9518 \mathrm{E}-04 \\
9.1451 \mathrm{E}-05 \\
4.2913 \mathrm{E}-05 \\
2.0154 \mathrm{E}-05 \\
9.4752 \mathrm{E}-06 \\
4.4590 \mathrm{E}-06 \\
2.0981 \mathrm{E}-06 \\
9.8740 \mathrm{E}-07 \\
4.6491 \mathrm{E}-07 \\
2 . i 912 \mathrm{E}-07 \\
1.9585 \mathrm{E}-07 \\
1.0000 \mathrm{0} \\
0\end{array}$ \\
\hline
\end{tabular}


Thas B-L

ZEBRA-2 70-EROUP TRRWSPOAT THEOAY SPECIRA

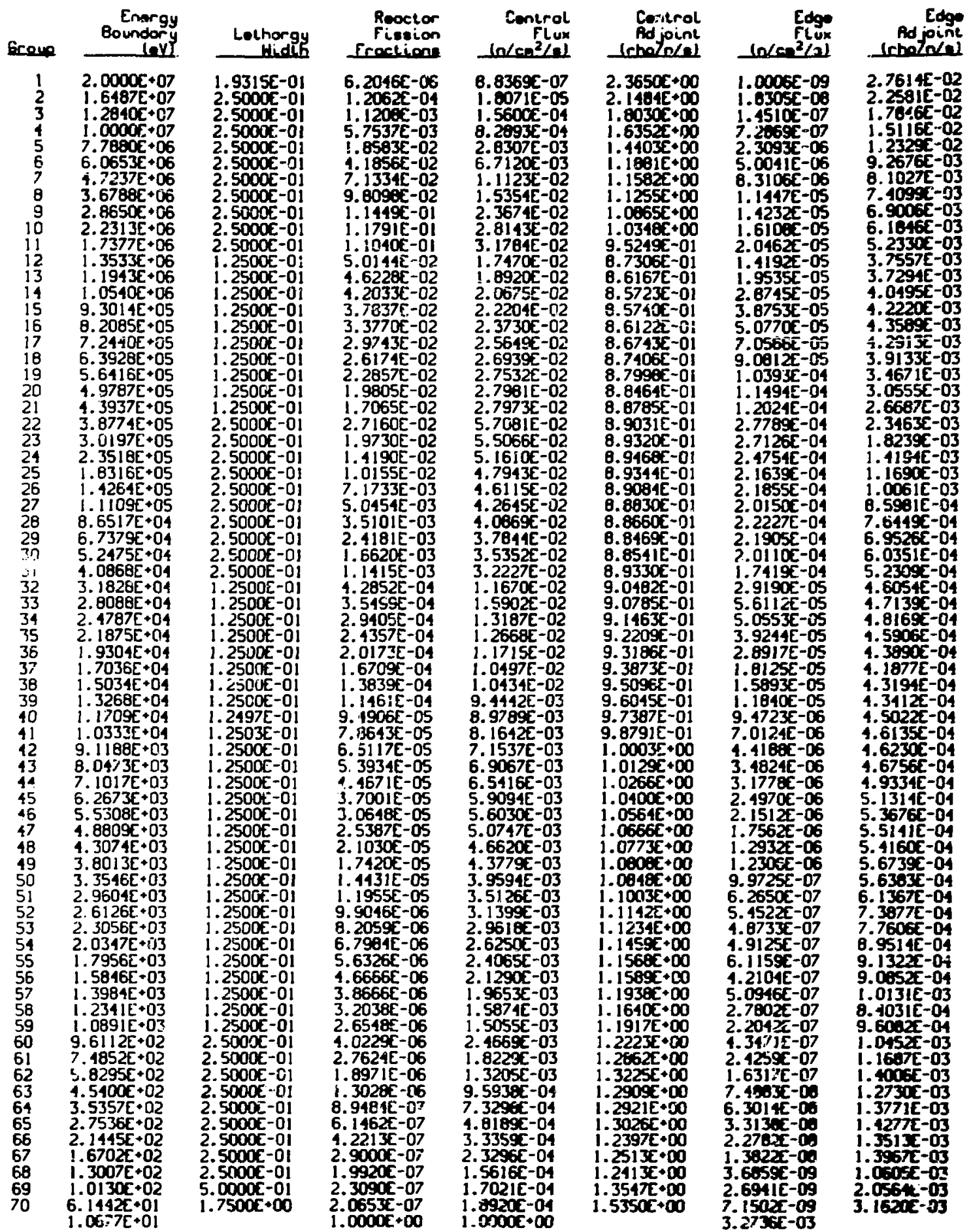


TABLE B-LI

ZEBRA-2 7O-GROUP ZONE FISSION FRACTIONS

\begin{tabular}{|c|c|c|c|c|}
\hline 040 & $\begin{array}{r}\text { Energy } \\
\text { Boundory } \\
\left(\mathbf{R}^{\prime}\right)\end{array}$ & $\begin{array}{c}\text { Lechor } \\
\text { Hie }\end{array}$ & Cor & Reflesto \\
\hline $\begin{array}{c}1 \\
2 \\
3 \\
4 \\
5 \\
6 \\
7 \\
8 \\
9 \\
9 \\
10 \\
11 \\
12 \\
13 \\
14 \\
15 \\
16 \\
17 \\
18 \\
19 \\
20 \\
21 \\
22 \\
23 \\
24 \\
25 \\
26 \\
27 \\
28 \\
29 \\
30 \\
31 \\
32 \\
33 \\
34 \\
35 \\
36 \\
37 \\
38 \\
39 \\
40 \\
41 \\
42 \\
13 \\
44 \\
45 \\
46 \\
47 \\
48 \\
49 \\
50 \\
51 \\
52 \\
53 \\
54 \\
55 \\
56 \\
57 \\
58 \\
59 \\
60 \\
61 \\
62 \\
63 \\
64 \\
65 \\
66 \\
67 \\
68 \\
69 \\
70\end{array}$ & 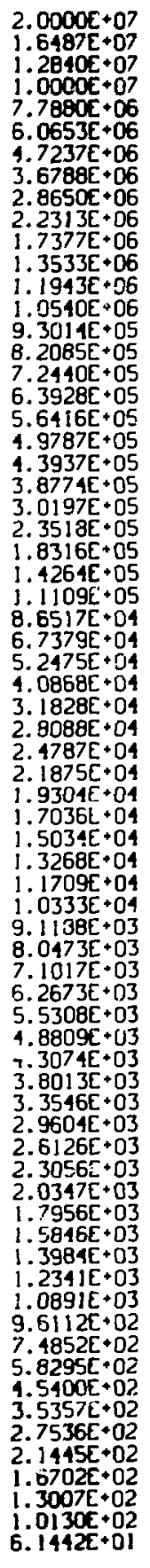 & 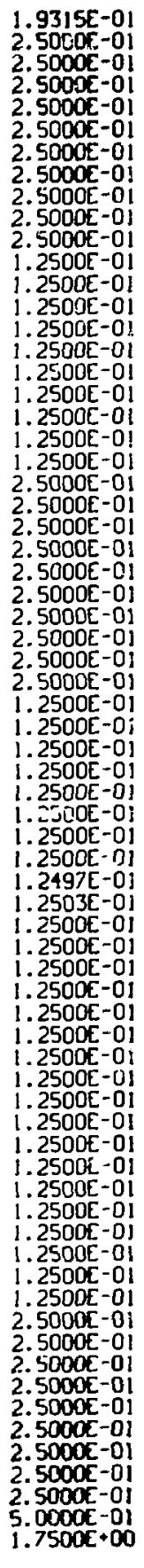 & 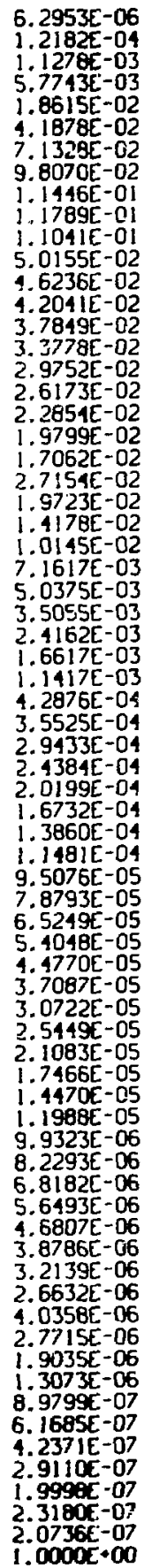 & 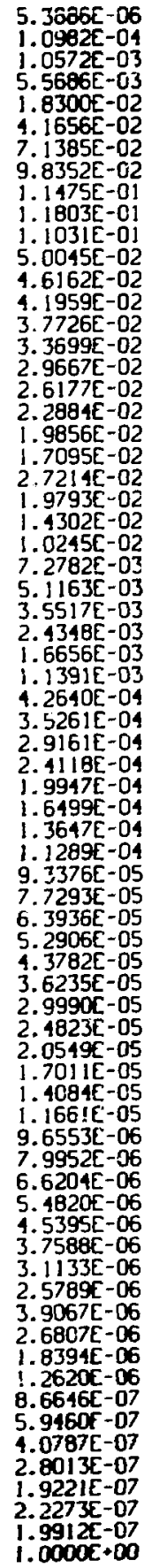 \\
\hline
\end{tabular}


TFBLE B-LII

ZEBAA-2 25-CROUP TAFWSPORT THEORY SPECTRA

\begin{tabular}{|c|c|}
\hline coup & $\begin{array}{r}\text { Enargy } \\
\text { Boundory } \\
\text { InVI }\end{array}$ \\
\hline $\begin{array}{r}1 \\
2 \\
3 \\
4 \\
5 \\
6 \\
7 \\
8 \\
9 \\
10 \\
11 \\
12 \\
13 \\
14 \\
15 \\
16 \\
17 \\
18 \\
19 \\
20 \\
21 \\
22 \\
23 \\
24 \\
25\end{array}$ & 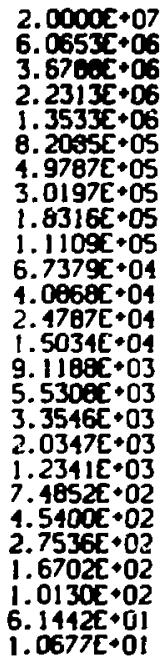 \\
\hline
\end{tabular}

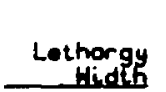

1. $1931 E+\infty$

5. $0000 \mathrm{C}-01$

5. $0000 \mathrm{c}-01$

5. $0000 \mathrm{c}-01$

5. $0000 \tau-01$

5. $0000 \mathrm{C}-01$

5.0000t-0i

5. $0000 \mathrm{c}-01$

5. $0000 \mathrm{c}-01$

5. 0000E-01

5. $0000 E-01$

5. $0000 \mathrm{c}-01$

5. $0000 \mathrm{C}-01$

5. 0000t-01

5. $0000[-0]$

5. $0000[-01$

5. co00r-01

5. $0000 \mathrm{r}-01$

5. 0000C-0!

5. $0000[-01$

5. $0000[-0]$

5. 0000e-01

5. $00005-01$

5. 0000E-01

1. 7500 E -00

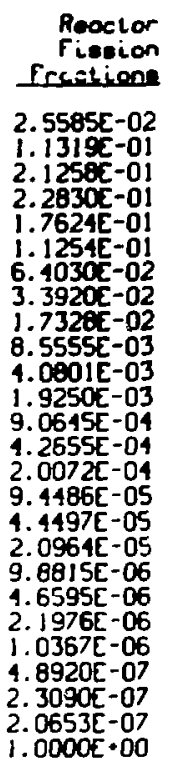

\begin{tabular}{|c|c|}
\hline $\begin{array}{r}\text { Centr } \\
f l \\
\ln \left\langle a n^{2}\right\rangle\end{array}$ & $\mathrm{C}$ \\
\hline 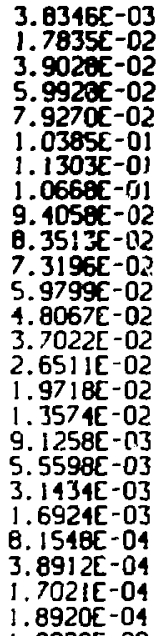 & $\begin{array}{r}8 \\
7 \\
1 \\
5 \\
9 \\
6 \\
6 \\
6 \\
6 \\
9 \\
1 \\
01 \\
1\end{array}$ \\
\hline
\end{tabular}

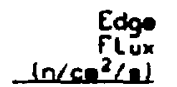

3. 20235-06

1. $3315 E-05$

2. 5600E-05

$3.6570 \mathrm{c}-05$

1. $.0122 E-04$

3. $16005-04$

5. $1307 E-04$

5. $1800 \mathrm{E}-04$

4. 3494E-04

4. 2377 E-04

4. $2015 E-04$

2. $594=0-04$

1. 3684E-04

1. $1216 E-05$

i. $3576 E-05$

6. $4312 E-06$

$2.65635-06$

$2.0333 E-06$

9. $3315 \mathrm{E}-07$

4. $0575 E-07$

1. 37905-07

5. $5920 \mathrm{c}-08$

1.7508E-08

$2.694\}$ E-09

7. $1502 \mathrm{E}-09$

3. $2736 E-03$
Edge

Ad jount

3. $5022 E-02$

6. 3711E-03

5. 24E6:-03

4. $1877 \mathrm{E}-03$

5.7792र -03

5.87975-03

2. $9601 E-[3$

$1.18955-03$

$7.9779 E-04$

5. 9576E-04

4. $7636 \mathrm{E}-04$

5. 3367 E-C4

6. 5962E-04

$6.5200 \mathrm{E}-04$

7. $1021 E-04$

8.0587E-04

$9.8745 \mathrm{t}-04$

$1.3681 E-03$

1. $0440 \mathrm{OE}-03$

$9.4236 E-04$

$9.71995-04$

1.01935-03

9. $01285-04$

$7.5423 E-04$

i. 1590 -0?

TABLE B-LIII

2EBRA-2 25-GROUP ZONE FISSION FRACTIONS

\begin{tabular}{|c|c|c|c|c|}
\hline Groug & $\begin{array}{r}\text { Enorgy } \\
\text { Boundory } \\
\text { InYI }\end{array}$ & $\begin{array}{r}\text { Lothorgy } \\
\text { Hodih }\end{array}$ & Core & Rerlecter \\
\hline $\begin{array}{l}1 \\
2 \\
3 \\
4 \\
5 \\
6 \\
7 \\
8 \\
9 \\
10 \\
11 \\
12 \\
13 \\
14 \\
15 \\
16 \\
17 \\
18 \\
19 \\
20 \\
21 \\
22 \\
23 \\
24 \\
25\end{array}$ & $\begin{array}{l}2.0000 E+07 \\
6.0653 E+06 \\
3.6788 E+06 \\
2.2313 E+06 \\
1.3533 E+06 \\
8.2085 E+05 \\
4.9787 E+05 \\
3.0197 E+05 \\
1.8315 E+05 \\
1.1109 E+05 \\
6.7379 E+04 \\
1.0668 E+04 \\
2.4787 E+04 \\
1.5034 E+04 \\
9.1186 E+03 \\
5.5308 E+03 \\
3.3546 E+03 \\
2.0347 E+03 \\
1.2341 E+03 \\
7.1852 E+02 \\
4.5400 E+02 \\
2.7536 E+02 \\
1.6702 E+02 \\
1.0130 E+02 \\
6.1412 E+01\end{array}$ & $\begin{array}{l}\text { 1. } 1931 E-00 \\
5.0000 E-01 \\
5.0000 E-01 \\
5.0000 E-01 \\
5.0000 E-01 \\
5.0000 E-01 \\
5.0000 E-01 \\
5.0000 E-01 \\
5.0000 E-01 \\
5.0000 E-01 \\
5.0000 E-01 \\
5.0000 E-01 \\
5.0000 E-01 \\
5.0000 E-01 \\
5.0000 E-0] \\
5.0000 E-01 \\
5.0000 E-01 \\
5.0000 E-01 \\
5.0000 E-01 \\
5.0000 E-01 \\
5.0000 E-01 \\
5.0000 E-0 ! \\
5.0000 E-01 \\
5.0000 E-01 \\
1.7500 E-01\end{array}$ & $\begin{array}{l}2.5645 E-02 \\
1.1321 E-01 \\
2.1253 E-01 \\
2.2630 E-01 \\
1.7628 E-01 \\
1.1256 E-01 \\
6.4015 E-02 \\
3.3900 E-02 \\
1.7307 E-02 \\
8.5429 E-03 \\
4.0779 E-03 \\
1.9257 E-03 \\
9.0747 E-01 \\
4.2726 E-04 \\
2.0115 E-04 \\
9.4720 E-05 \\
4.4620 E-05 \\
2.1027 E-05 \\
9.9129 E-06 \\
4.6750 E-06 \\
2.2053 E-06 \\
1.0106 E-06 \\
1.9107 E-07 \\
2.3190 E-07 \\
2.0736 E-07 \\
1.0000 E+00\end{array}$ & $\begin{array}{l}2.504 ! E-02 \\
1.1304 E-G 1 \\
2.1310 E-01 \\
2.2834 E-01 \\
1.7589 E-01 \\
1.1243 E-01 \\
6.4165 E-02 \\
3.4094 E-02 \\
1.7523 E-02 \\
8.0680 E-03 \\
1.1004 E-03 \\
1.9181 E-03 \\
8.9724 E-04 \\
4.2004 E-04 \\
1.9686 E-04 \\
9.2373 E-05 \\
4.3395 E-05 \\
2.040 ! E-05 \\
9.5989 E-76 \\
1.5201 E-05 \\
2.1284 E-06 \\
1.0025 E-06 \\
4.7233 E-07 \\
2.2273 E-07 \\
1.9912 E-07 \\
1.0000 E \cdot 00\end{array}$ \\
\hline
\end{tabular}


TFBLE B-LIV

ZPPR-2 70-GROUP TRFWSPORT THEORY SPECTPA

\begin{tabular}{|c|c|c|c|c|c|c|c|}
\hline cone & 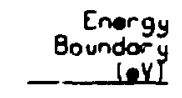 & $\begin{array}{r}\text { Lethorgj } \\
\text { Nidif }\end{array}$ & $\begin{array}{r}\text { Reoctor } \\
\text { Fieston } \\
\text { Froction? }\end{array}$ & $\begin{array}{r}\text { Control } \\
\text { Flux } \\
\ln / c^{2} m^{2} / n l \\
\end{array}$ & $\begin{array}{r}\text { Control } \\
\text { Ad jount } \\
\text { lrholntel }\end{array}$ & $\begin{array}{r}\text { Edge } \\
f(u x) \\
\ln \left(\operatorname{con}^{2} / \mathrm{a}\right)\end{array}$ & $\begin{array}{r}\text { Edg } \\
\text { Rd poun } \\
\text { tchotola }\end{array}$ \\
\hline $\begin{array}{l}1 \\
2 \\
3 \\
1 \\
5 \\
6 \\
7 \\
8 \\
0 \\
10 \\
11 \\
12 \\
13 \\
14 \\
15 \\
16 \\
17 \\
18 \\
19 \\
20 \\
21 \\
22 \\
23 \\
24 \\
25 \\
26 \\
27 \\
28 \\
29 \\
30 \\
31 \\
32 \\
33 \\
34 \\
35 \\
36 \\
37 \\
38 \\
39 \\
40 \\
41 \\
42 \\
43 \\
44 \\
45 \\
46 \\
17 \\
48 \\
49 \\
50 \\
51 \\
52 \\
53 \\
51 \\
55 \\
56 \\
57 \\
58 \\
59 \\
60 \\
61 \\
62 \\
63 \\
64 \\
66 \\
69 \\
69 \\
69\end{array}$ & $\begin{array}{l}2.0000 E+07 \\
1.6487 E+07 \\
1.2640 E+07 \\
1.0000 E+07 \\
7.7880 E+06 \\
6.0653 E+06 \\
4.7237 E+06 \\
3.6786 E+06 \\
2.8650 E+06 \\
2.2313 E+06 \\
1.7377 E+06 \\
1.3533 E+06 \\
1.1943 E+06 \\
1.0540 E+06 \\
9.3014 E+05 \\
8.2085 E+05 \\
7.2440 E+05 \\
6.3928 E+05 \\
5.6416 E+05 \\
4.9787 E+05 \\
4.3937 E+05 \\
3.0774 E+05 \\
3.0197 E+05 \\
2.3518 E+05 \\
1.8316 E+05 \\
1.4264 E+05 \\
1.1109 E+05 \\
8.6517 E+04 \\
6.7379 E+04 \\
5.2475 E+04 \\
4.0868 E+04 \\
3.1828 E+04 \\
2.8088 E+04 \\
2.4787 E+04 \\
2.1875 E+04 \\
1.9304 E+04 \\
1.7036 E+04 \\
1.5034 E+04 \\
1.3268 E+04 \\
1.1709 E+04 \\
1.0333 E+04 \\
9.1188 E+03 \\
8.0473 E+03 \\
7.1017 E+03 \\
6.2673 E+03 \\
5.5308 E+03 \\
4.8809 E+03 \\
4.3074 E+03 \\
3.8013 E+03 \\
3.3546 E+03 \\
2.9604 E+03 \\
2.6126 E+03 \\
2.3056 E+03 \\
2.0347 E+03 \\
1.7956 E+03 \\
1.5846 E+03 \\
1.3984 E+03 \\
1.2341 E+03 \\
1.0891 E+03 \\
9.6112 E+02 \\
7.4852 E+02 \\
5.8295 E+02 \\
4.5400 E+02 \\
3.5357 E+02 \\
2.7536 E+02 \\
2.1445 E+02 \\
1.6702 E+02 \\
1.3007 E+02 \\
1.0130 E+02 \\
6.1442 E+01 \\
1.0677 E+01\end{array}$ & $\begin{array}{l}1.9315 E-01 \\
2.5000 E-01 \\
2.5000 E-01 \\
2.5000 E-01 \\
2.5000 E-01 \\
2.5000 E-01 \\
2.5000 E-01 \\
2.5000 E-01 \\
2.5000 E-01 \\
2.5000 E-01 \\
2.5000 E-01 \\
1.2500 E-01 \\
1.2500 E-01 \\
1.2500 E-01 \\
1.2500 E-01 \\
1.2500 E-01 \\
1.2500 E-01 \\
1.2500 E-01 \\
1.2500 E-01 \\
1.2500 E-01 \\
1.2500 E-01 \\
2.5000 E-01 \\
2.5000 E-01 \\
2.5000 E-01 \\
2.5000 E-01 \\
2.5000 E-01 \\
2.5000 E-01 \\
2.5000 E-01 \\
2.5000 E-01 \\
2.5000 E-01 \\
2.5000 E-01 \\
1.2500 E-01 \\
1.2500 E-01 \\
1.2500 E-0 ! \\
1.2500 E-01 \\
1.2500 E-01 \\
1.2500 E-01 \\
1.2500 E-01 \\
1.2500 E-01 \\
1.24975-01 \\
1.2503 E-01 \\
1.2500 E-01 \\
2.5000 E-01 \\
2.5000 E-01 \\
1.2500 E-01 \\
1.7500 E-01 \\
1.2500 E-01 \\
1.2500 E-01 \\
1.2500 E-01 \\
1.2500 E-01 \\
1.2500 E-01 \\
1.2500 E-01 \\
1.2500 E-01 \\
1.2500 E-01 \\
1.2500 E-01 \\
1.2500 E-01 \\
1.2500 E-01 \\
1.2500 E-01 \\
1.2500 E-01 \\
1.2500 E-01 \\
1.2500 E-01 \\
1.2500 E-01 \\
2.5000 E-01 \\
2.5000 E-01 \\
2.500 E-01 \\
2.500 E-01 \\
2\end{array}$ & 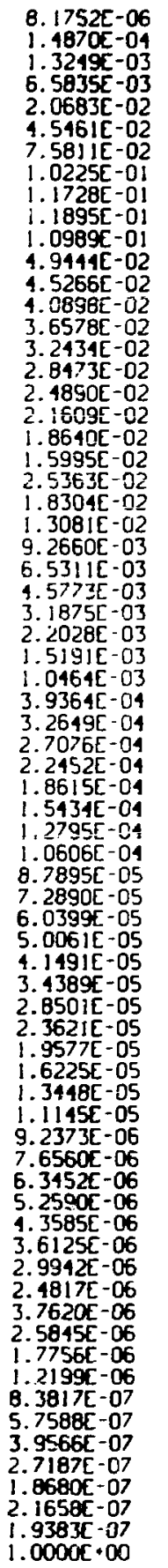 & 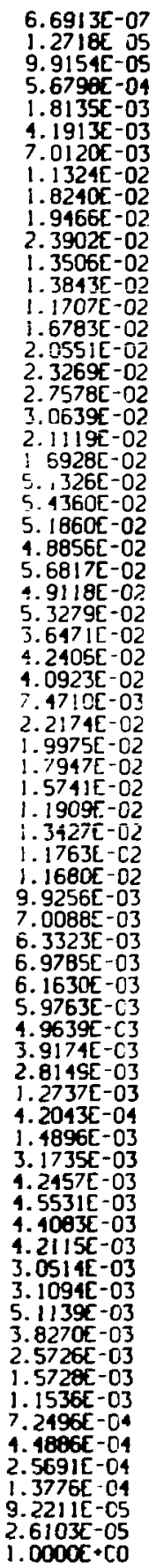 & 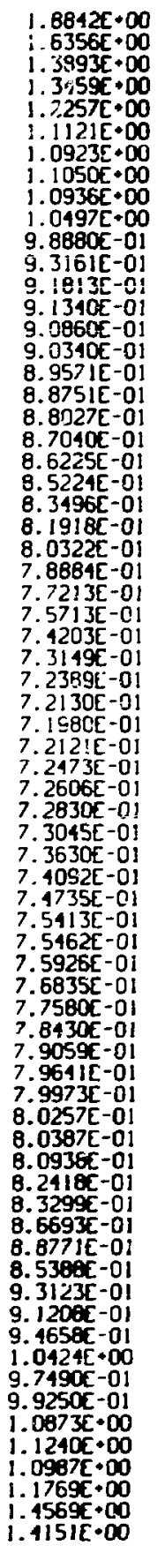 & 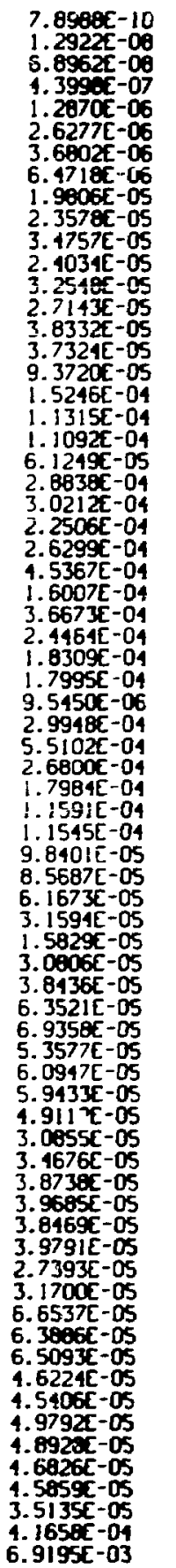 & 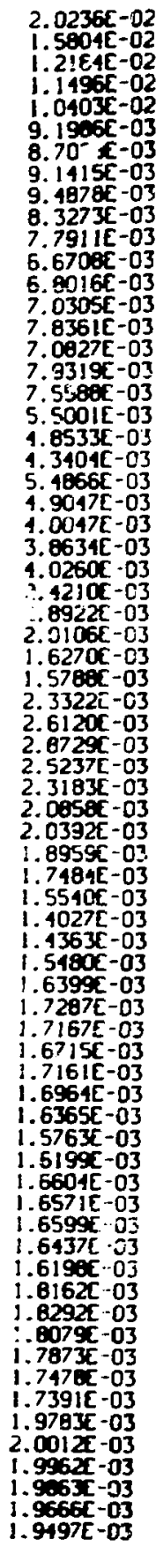 \\
\hline
\end{tabular}


Troue B-LV

2PPR-2 70-CROUP ZONE FISSION FRRCTIONS

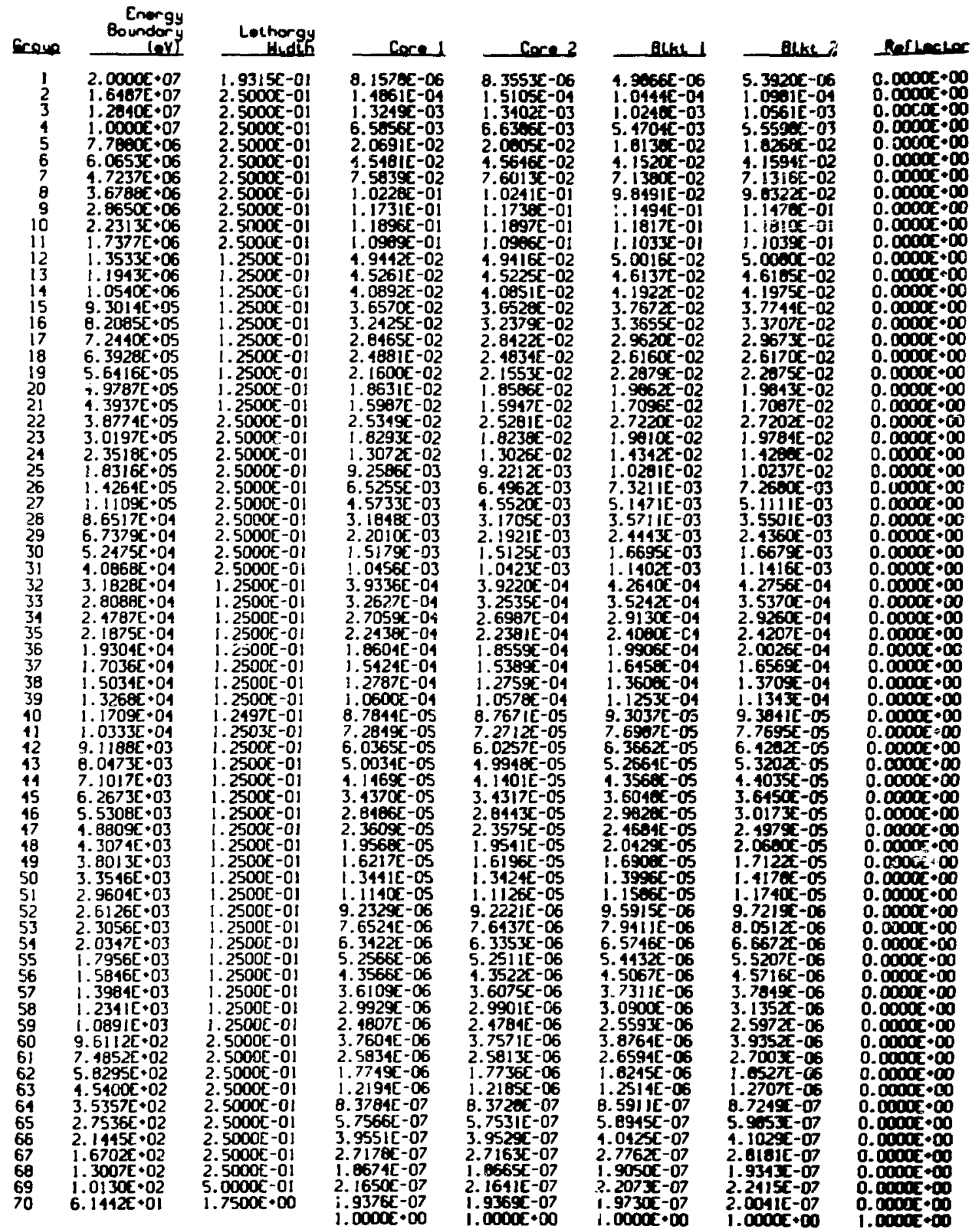


TFBLE B-LVI

2PPR-2 25-GROUP TRAW5PORT THEORY SPECTRR

\begin{tabular}{|c|c|}
\hline Eroug & $\begin{array}{r}\text { Energy } \\
\text { Boundory } \\
\text { (nv) }\end{array}$ \\
\hline $\begin{array}{l}1 \\
2 \\
3 \\
4 \\
5 \\
6 \\
7 \\
8 \\
9 \\
10 \\
11 \\
12 \\
13 \\
14 \\
15 \\
16 \\
17 \\
18 \\
19 \\
20 \\
21 \\
22 \\
23 \\
24 \\
25\end{array}$ & 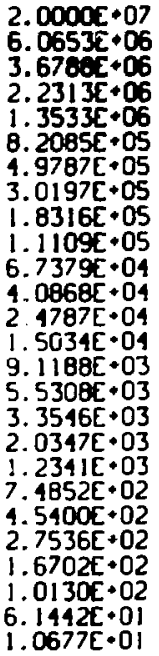 \\
\hline
\end{tabular}

\begin{tabular}{|c|c|c|}
\hline $\begin{array}{r}\text { Lethorgy } \\
\text { HLdih }\end{array}$ & $\begin{array}{r}\text { Resctar } \\
\text { Fiselan } \\
\text { Fraction }\end{array}$ & $\begin{array}{r}\text { Centrol } \\
F l u x \\
\ln / \mathrm{cm}^{2} / \mathrm{sil} \\
\end{array}$ \\
\hline $\begin{array}{l}1.1931 E+00 \\
5.0000 E-01 \\
5.0000 E-01 \\
5.0000 E-01 \\
5.0000 E-01 \\
5.0000 E-01 \\
5.0000 E-01 \\
5.0000 E-01 \\
5.0000 E-01 \\
5.0000 E-01 \\
5.0000 E-01 \\
5.0000 E-01 \\
5.0000 E-01 \\
5.0000 E-01 \\
5.0000 E-01 \\
5.0000 E-01 \\
5.0000 E-01 \\
5.0000 E-01 \\
5.0000 E-01 \\
5.0000 E-01 \\
5.0000 E-01 \\
5.0000 E-01 \\
5.0000 E-01 \\
5.0000 E-01 \\
1.7500 E-01\end{array}$ & $\begin{array}{l}2.8748 E-02 \\
1.2127 E-01 \\
2.1953 E-01 \\
2.2881 E-01 \\
1.7219 E-01 \\
1.0741 E-01 \\
5.9998 E-02 \\
3.1385 E-02 \\
1.5797 E-02 \\
7.7648 E-03 \\
3.7219 E-03 \\
1.7665 E-03 \\
8.3578 E-04 \\
3.9479 E-04 \\
1.8634 E-04 \\
8.7925 E-05 \\
1.1486 E-05 \\
1.9575 E-05 \\
9.2379 E-0 E \\
1.3601 E-06 \\
2.0580 E-06 \\
9.7154 E-07 \\
1.5868 E-07 \\
2.1658 E-07 \\
1.9383 E-07 \\
1.0000 E+00\end{array}$ & $\begin{array}{l}2.4940 E-03 \\
1.1203 E-02 \\
2.9564 E-02 \\
1.3368 E-02 \\
5.5840 E-02 \\
1.0204 E-01 \\
\text { B. } 9373 E-02 \\
1.0622 E-01 \\
1.0567 E-01 \\
1.0240 E-01 \\
7.8877 E-02 \\
7.0568 E-02 \\
6.5572 E-02 \\
1.6796 E-02 \\
2.6483 E-02 \\
1.7673 E-02 \\
6.3572 E-03 \\
1.7419 E-02 \\
1.1275 E-02 \\
6.3996 E-03 \\
2.7261 E-03 \\
1.1738 E-03 \\
3.946 B E-04 \\
9.2211 E-05 \\
2.6103 E \\
1.0000 E-00\end{array}$ \\
\hline
\end{tabular}

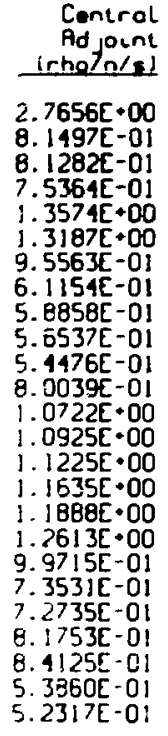

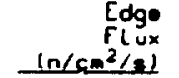

1. $8097 E-06$ $6.3080 \tau-06$

2. $627 \mathrm{BC}-05$ $5.8335 \mathrm{E}-05$ 1.2206E-04 $3.9665 E-04$ 4. $6055 E-04$ $5.2718 \mathrm{E}-04$ 7. $1666 \mathrm{E}-04$ 5. $26 B 0 t-04$ 4. $2773 \mathrm{E}-04$ 4. 8898E-04 $1.1148[-03$ $3.6121 \mathrm{E}-04$ 1. $1667 E-04$ $2.1740 E-04$ 1.7408E-04 $1.5668 \mathrm{C}-04$ 1. $2563 \mathrm{E}-04$ 1. 2898[-04 $9.1630 \mathrm{C}-05$ 9. 8720E-05 9. $2685[-05$ $3.5 ! 35 E-05$ 4. $1658 E-04$ 6. $9: 95[-03$

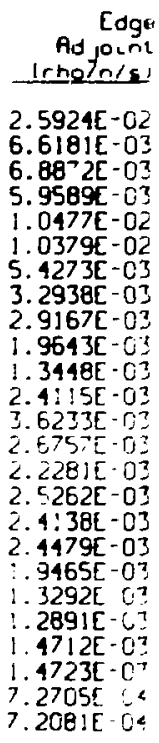

Eoge irmotols

$2.5924 E-02$ 6.618]E-03 6. $88^{7} 2 \mathrm{E}-03$ 列 1. $0379 E-02$ 5. $4273 E-03$ 3. $2938[-0]$ 2.9167E-0? $.9643 E-63$ 4:15E-0?

$62735-r^{2}$ 2. $5262 \mathrm{E}-0$ ? . 4:38L-03 4479[-0] 2891E-C? $4712[-0 ?$ 7.27055 \& .

TRGLE B-LVIJ

2PPR-2 25-GROLP ZONE FISSION FRACTJONS

\begin{tabular}{|c|c|}
\hline Group & $\begin{array}{r}\text { Enorgy } \\
\text { Boundory } \\
\text { (nVI) }\end{array}$ \\
\hline $\begin{array}{l}1 \\
2 \\
3 \\
4 \\
5 \\
6 \\
7 \\
8 \\
9 \\
10 \\
11 \\
12 \\
13 \\
14 \\
15 \\
16 \\
17 \\
18 \\
19 \\
20 \\
21 \\
22 \\
23 \\
24 \\
24\end{array}$ & $\begin{array}{l}2.0000 E+07 \\
6.0653 E+06 \\
3.6780 E+06 \\
2.2313 E+06 \\
1.3533 E+05 \\
8.2005 E+05 \\
4.9787 E+05 \\
3.0197 E+05 \\
1.0315 E+05 \\
1.1109 E+05 \\
6.7379 E+04 \\
4.0868 E+04 \\
2.1787 E+04 \\
1.5034 E+04 \\
9.1180 E+03 \\
5.5308 E+03 \\
3.3546 E+03 \\
2.0347 E+03 \\
1.2311 E+03 \\
7.1852 E+02 \\
1.5400 E \cdot 02 \\
2.7536 E+02 \\
1.6702 E+02 \\
1.0130 E+02 \\
5.1442 E+01\end{array}$ \\
\hline
\end{tabular}

\section{Lethorgy
Ntdth}

1. $1931 E+00$

$5.00005-01$

$5.0000 c-01$

5. OMDOE-01

5. 0000 - -01

5.0000E-01

5. $0000 \mathrm{E}-01$

5.0000E-01

5. $0000 \mathrm{E}-01$

5. $0000 \mathrm{E}-0 \mathrm{i}$

5. $0000 \mathrm{E}-01$

5. $0000 \mathrm{C}-01$

$5.0000 \mathrm{C}-01$

5. $0000[-01$

S. $0000[-0]$

5. $00005-01$

5. OOODE-01

5. O000E-01

5. 0000E-01

5. O0OOE-O

5. $0000 \mathrm{C}-0$

5. O0DOE-01

5. $0000 E-0$

5. 0000E-01

\begin{tabular}{r} 
Core 1 \\
\hline $2.8759 E-02$ \\
$1.2132 E-01$ \\
$2.1959 E-01$ \\
$2.2885 E-01$ \\
$1.7217 E-01$ \\
$1.0737 E-01$ \\
$5.9967 E-02$ \\
$3.1365 E-02$ \\
$1.5784 E-02$ \\
$7.7581 E-03$ \\
$3.7190 E-03$ \\
$1.7652 E-03$ \\
$8.3524 E-04$ \\
$3.9456 E-04$ \\
$1.8624 E-04$ \\
$8.7860 E-05$ \\
$1.1466 E-05$ \\
$1.9566 E-05$ \\
$9.2339 E-06$ \\
$4.3583 E-06$ \\
$2.0572 E-06$ \\
$9.7118 E-07$ \\
$1.5851 E-07$ \\
$2.1650 E-07$ \\
$1.9376 E-07$ \\
$1.0000 E+00$
\end{tabular}

Core 2

2. $8941 E-02$

$1.2166 E-01$

2. $1900 \mathrm{t}-01$

$2.2883 E-01$

$1.7202 E-01$

1. 0719E-01

$5.9814 E-02$

3. $1264 E-02$

$1.5717 \mathrm{E}-02$

7.7226E-03

$3.7046 E-03$

1.7590E-03

8. 3316E-04

$3.9375 E-04$

1. $8592 \mathrm{~L}-04$

$8.7755 E-05$

4. $1416 E-05$

1. 9546E-05

$9.2256 \mathrm{E}-06$

4. 3549E- 06

2. $0558 E-06$

9. $7061 \%-07$

4. $5828 E-97$

2. $1641 E-07$

$1.93695-07$

1. $0000 \mathrm{E}+00$

\section{Blkt}

2. $47435-02$

$1.1290 E-01$

$2.1343 E-01$

2. $2850 E-01$

$1.7575 E-01$

1. 123$] \mathrm{E}-01$

6. $1178 E-02$

3.4152E-02

1.7602E-02

B. $71825-03$

4. 1$] 385-03$

1. $9190 E-03$

8. 9574E-04

4. $1863 t-04$

1. $9594 \mathrm{E}-04$

9. 1849t-05

4. $3114 E-05$

$2.0256 E-05$

9.5257E-06

4. 1838[-06

$2.1105 E-06$

$9.9370[-07$

4. $6812 \varepsilon-07$

2. $2073 E-07$

1. $9730 \mathrm{E}-07$

1. anOOE +00

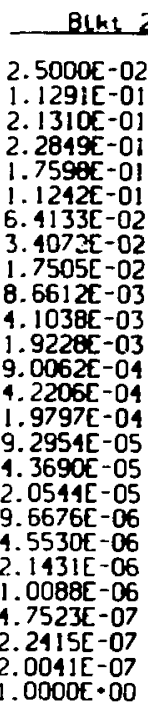

Reflector

$0.0000 E \cdot 00$

$0.0000 E \cdot 00$

D. OOCOE.0O

$0.0000 E+00$

$0.0000 E+00$

0.0000 - 00

0. ODOOE. 00

$0.0000 E \cdot 00$

$0.0000 E \cdot 00$

D. OOODE - $\mathrm{CO}$

$0.0000 \mathrm{0} * 0 \mathrm{O}$

$0.0000 E \cdot 00$

0. 0000E-00

$0.0000 E \cdot 0$

$0.0000[\cdot 0]$

$0.0000 \mathrm{t}-0 \mathrm{O}$

$0.0000 E \cdot 00$

O. OCOOE-0O

$0.0000 E-00$

0. OOOOE.00

$0.00005 \cdot 00$

$0.0000 \mathrm{r} \cdot 00$

$0.00005 \cdot 00$

$0.0000 \mathrm{0}+00$

$0.0000<+00$

1. $000 \mathrm{OE}+\mathrm{CO}$ 
THOLE B-LVIII

ZPR-6-7 70-GROU TAPWSPORT THEORY SPECTRA

\begin{tabular}{|c|c|c|c|c|c|c|c|}
\hline ong & $\begin{array}{r}\text { Energy } \\
\text { Boundory } \\
\text { feyl }\end{array}$ & $\begin{array}{r}\text { Lethorgy } \\
\text { Hidlh }\end{array}$ & $\begin{array}{r}\text { Reoclor } \\
\text { Fievion } \\
\text { Proctions }\end{array}$ & $\begin{array}{r}\text { Centrol } \\
\text { flux } \\
\ln \left(\operatorname{sen}^{2} / x\right) \\
\end{array}$ & $\begin{array}{r}\text { Centrol } \\
\text { Ad pint } \\
\text { Irhotolnl }\end{array}$ & $\ln \left\langle\operatorname{con}^{2}\right.$ & $\begin{array}{r}\text { Edge } \\
\text { Ad joint } \\
\text { laboln/al }\end{array}$ \\
\hline $\begin{array}{l}1 \\
2 \\
3 \\
1 \\
5 \\
6 \\
7 \\
8 \\
9 \\
10 \\
11 \\
12 \\
13 \\
14 \\
15 \\
16 \\
17 \\
18 \\
19 \\
20 \\
21 \\
22 \\
23 \\
24 \\
25 \\
26 \\
27 \\
28 \\
29 \\
30 \\
31 \\
32 \\
33 \\
34 \\
35 \\
36 \\
37 \\
38 \\
39 \\
10 \\
41 \\
42 \\
43 \\
44 \\
45 \\
16 \\
47 \\
48 \\
49 \\
50 \\
51 \\
52 \\
53 \\
54 \\
55 \\
56 \\
57 \\
58 \\
59 \\
60 \\
61 \\
62 \\
63 \\
64 \\
65 \\
66 \\
67 \\
68 \\
69 \\
70\end{array}$ & 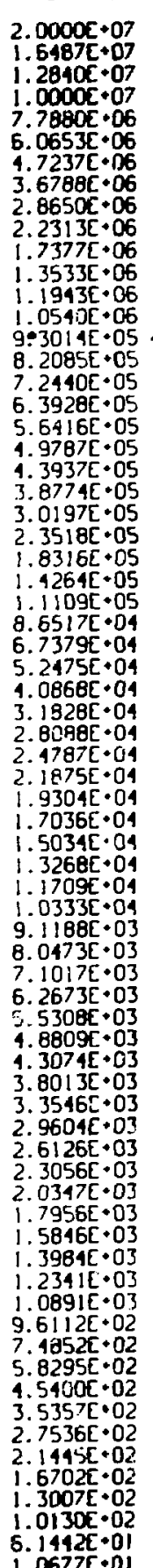 & $\begin{array}{l}1.9315 E-01 \\
2.5000 E-01 \\
2.5000 E-01 \\
2.5000 E-01 \\
2.5000 E-01 \\
2.5000 E-01 \\
2.5000 E-01 \\
2.5000 E-01 \\
2.5000 E-01 \\
2.5000 E-01 \\
2.5000 E-01 \\
1.2500 E-01 \\
1.2500 E-01 \\
1.2500 E-01 \\
1.2500 E-01 \\
1.2500 E-01 \\
1.2500 E-01 \\
1.2500 E-01 \\
1.2500 E-01 \\
1.2500 E-01 \\
1.2500 E-01 \\
2.5000 E-01 \\
2.5000 E-01 \\
2.5000 E-01 \\
2.5000 E-01 \\
2.5000 E-01 \\
2.5000 E-01 \\
2.5000 E-01 \\
2.5000 E-01 \\
2.5000 E-01 \\
2.5000 E-01 \\
1.2500 E-01 \\
1.2500 E-01 \\
1.2500 E-01 \\
1.2500 E-01 \\
1.2500 E-01 \\
1.2500 E-01 \\
1.2500 E-01 \\
1.2500 E-01 \\
1.2497 E-01 \\
1.2503 E-01 \\
1.2500 E-01 \\
1.2500 E-01 \\
1.2500 E-01 \\
1.2500 E-01 \\
1.2500 E-01 \\
1.2500 E-01 \\
1.2500 E-01 \\
1.2500 E-01 \\
1.2500 E-01 \\
1.2500 E-01 \\
1.2500 E-01 \\
1.2500 E-01 \\
1.2500 E-01 \\
1.2500 E-01 \\
1.2500 E-01 \\
1.250 C E-01 \\
1.250 C E-01 \\
1.2500 E-01 \\
2.5000 E-01 \\
2.500 \mathrm{E}-01 \\
2.5000 E-01 \\
2.5000 E-01 \\
2.5000 E-01 \\
2.5000 E-01 \\
2.5000 E-01 \\
2.5000 E-01 \\
2.5000 E-01 \\
5.0000 E-01 \\
1.7500 E-00\end{array}$ & 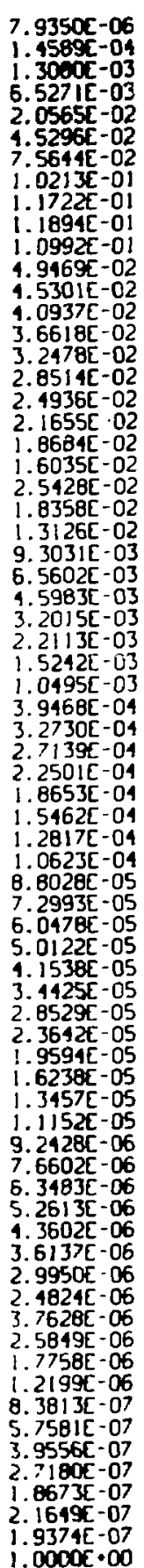 & 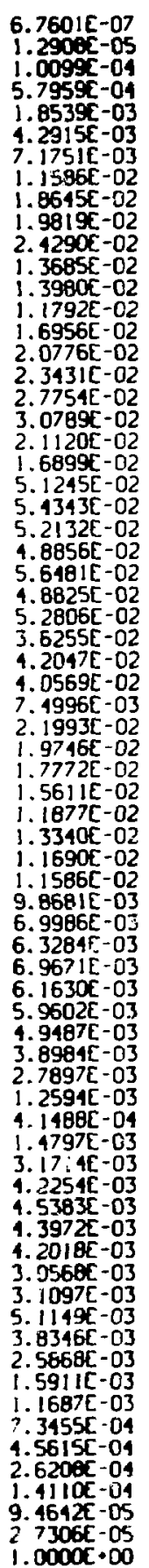 & 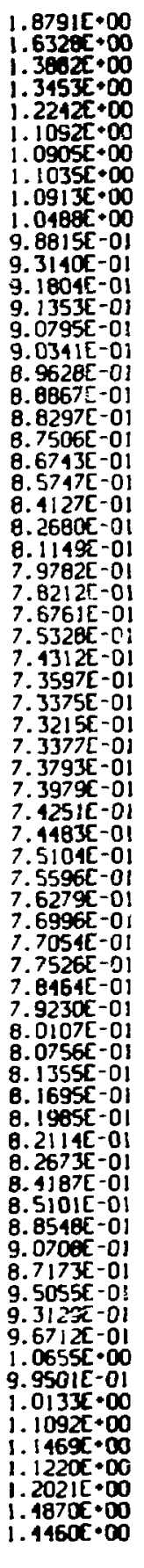 & 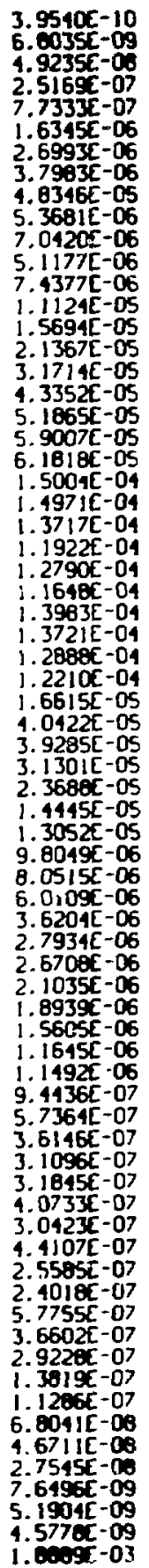 & 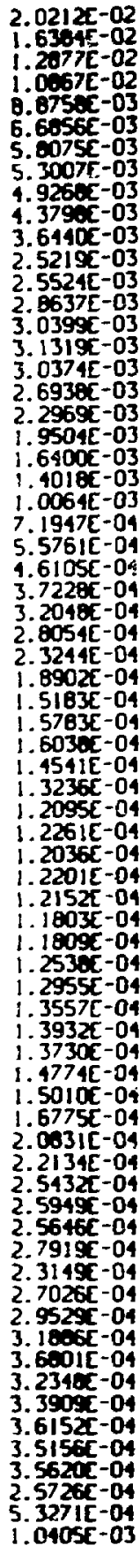 \\
\hline
\end{tabular}


TABLE B-LIX

ZPR-6 7 70-GROUP ZONE FISSION FRACTIONS

\begin{tabular}{|c|c|c|c|c|}
\hline -oup & $\begin{array}{r}\text { Enorgy } \\
\text { Boundory } \\
\text { InYI }\end{array}$ & $\begin{array}{r}\text { Leehorgy } \\
\text { budih }\end{array}$ & Cors & Reflector \\
\hline $\begin{array}{l}1 \\
2 \\
3 \\
4 \\
5 \\
6 \\
7 \\
8 \\
9 \\
10 \\
11 \\
12 \\
13 \\
14 \\
15 \\
16 \\
17 \\
18 \\
19 \\
20 \\
21 \\
22 \\
23 \\
24 \\
25 \\
26 \\
27 \\
28 \\
29 \\
30 \\
31 \\
32 \\
33 \\
34 \\
35 \\
36 \\
37 \\
38 \\
39 \\
40 \\
41 \\
42 \\
43 \\
44 \\
45 \\
46 \\
47 \\
48 \\
49 \\
50 \\
51 \\
52 \\
53 \\
54 \\
55 \\
35 \\
57 \\
58 \\
59 \\
60 \\
61 \\
62 \\
63 \\
64 \\
65 \\
56 \\
67 \\
70 \\
70 \\
6\end{array}$ & 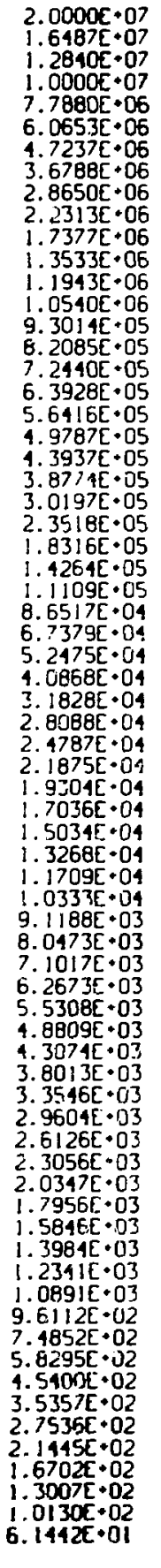 & 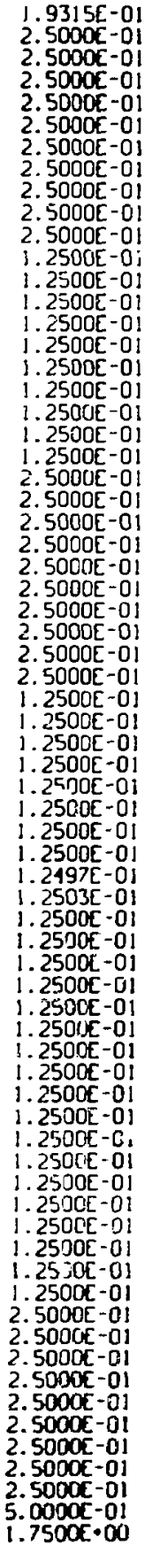 & 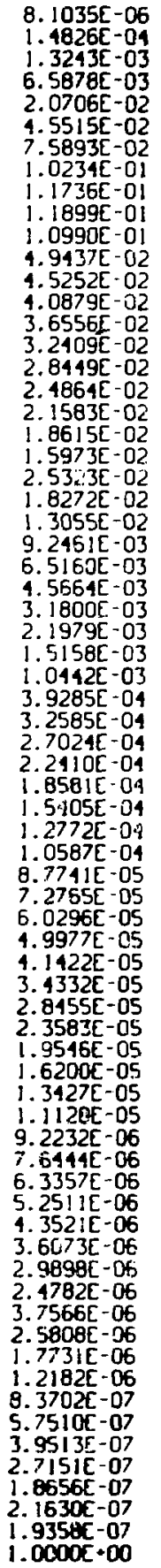 & 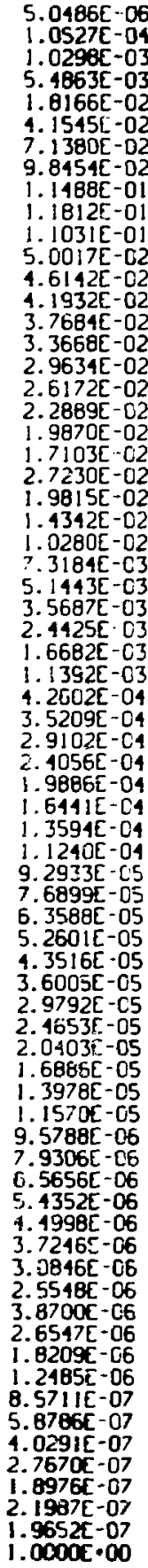 \\
\hline
\end{tabular}


TFaLE B-LX

2PR-6-7 25-GROUP TRANSPORT THEORY SPECTRA

\begin{tabular}{|c|c|}
\hline eng & $\begin{array}{r}\text { Energy } \\
\text { 8oundory } \\
\text { levI } \\
\end{array}$ \\
\hline $\begin{array}{r}1 \\
2 \\
3 \\
1 \\
5 \\
6 \\
7 \\
8 \\
9 \\
10 \\
11 \\
12 \\
13 \\
14 \\
15 \\
16 \\
17 \\
18 \\
19 \\
20 \\
21 \\
22 \\
23 \\
21 \\
\end{array}$ & 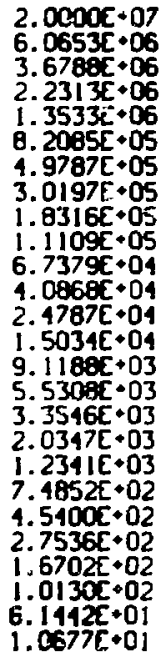 \\
\hline
\end{tabular}

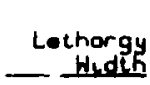

1. $1931 E+00$

5. $0000 \mathrm{C}-01$

5. $0000 \mathrm{c}-01$

5. $0000 \mathrm{C}-01$

5. 0000E-01

5. $0000 \mathrm{x}-01$

5. $0000 t^{-}-01$

5. $0000 \mathrm{E}-01$

5. $00005-01$

5. $0000 E-01$

5. $0000 E-01$

5. $0000 E-01$

$5.0000 E-01$

S. ODOOE-01

5. $0000[-0:$

$5.0000[-0]$

5. $0000[-0$ :

$5.0000[-01$

5. $0000[-0]$

5. $00005-01$

$5.0000 \mathrm{c}-01$

5. 0000E-0!

5.0000t-01

5.0000E-0

1. $7500 c+00$

\begin{tabular}{r} 
Reoctor \\
Fiescon \\
Frocleone \\
\hline $2.8554 E-02$ \\
$1.2094 E-01$ \\
$2.1935 E-01$ \\
$2.2866 E-01$ \\
$1.7233 E-01$ \\
$1.0756 E-01$ \\
$6.0146 E-02$ \\
$3.1184 E-02$ \\
$1.5863 E-02$ \\
$7.7997 E-03$ \\
$3.7356 E-03$ \\
$1.7714 E-03$ \\
$8.3755 E-04$ \\
$3.9542 E-04$ \\
$1.8656 E-04$ \\
$8.8002 E-05$ \\
$1.1513 E-05$ \\
$1.9584 E-05$ \\
$9.2403 E-06$ \\
$1.3607 E-06$ \\
$2.0580 E-06$ \\
$9.7136 E-07$ \\
$1.5853 E-07$ \\
$2.1649 E-07$ \\
$1.9374 E-07$ \\
$1.0000 E+0 C$
\end{tabular}

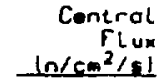

2. 5480E-03

1. $1467 \mathrm{E}-02$

$3.0231 E-02$

$4.1110=-02$

5. 6413E-02

$1.0275 E-0$ !

8. 9264E-02

1. $0647 \mathrm{E}-0$ !

1. $0534 E-0$ !

1. $0163 \mathrm{E}-01$

$7.8302 E-02$

$7.0062 \mathrm{E}-02$

6. 5007[-02

4. 6484 E- 02

2.6457E-02

1.7597E-02

6. $3254[-03$

1.73635-02

1. $1281 \mathrm{E}-02$

6. $4214[-03$

$2.7597 \mathrm{E}-03$

i. $19075-03$

1.0318E-04

9. $4642 E-05$

$2.73065-05$

1. OOODE.00

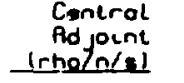

$2.76135 \cdot 00$

8. $1318[-01$

8. $1136 \bar{E}-01$

7. $5303 E-01$

$1.3571 E \cdot 00$

1. $32022 \cdot 00$

9.6] $15[-0]$

6. $1665 \bar{E}-01$

5. $9893 \mathrm{~K}-01$

5. $7290 \mathrm{~L}-01$

5. $5319 E-01$

8. $1308 \mathrm{C}-01$

1. $0920 E \cdot 00$

1. $1144 \mathrm{E}+00$

$1.14615 \cdot 00$

1. $1883 E+00$

1. $2143 \mathrm{E} \cdot 0 \mathrm{O}$

1. $2885[\cdot 00$

$1.01795 \cdot 00$

7. $5143[-01$

$7.42425-0$ !

8. 3403[-0]

8. $5917 \mathrm{E}-01$

5. 4970E-01

5. 3456E-01

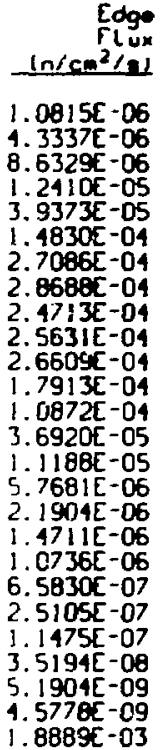

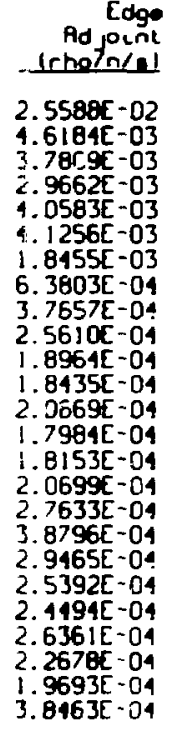

Edgo 4. 61845-03 3. $78 \mathrm{gr},-03$ 1. 0583E-03 4. $1256[-03$ 1. 8455[-03 3. $7657 \mathrm{E}-04$ 2. 5610C-04 1.8964[-04 345-04 .7984E-04 .8153[-04 2. 0699L-04 2. 7633E-04 $3.87965-04$
$2.9465 E-04$ 2. 5392E-04 2. 4494E-04 $6361 E-04$ 1. $9693 E-04$

$3.8463 E-04$

TRBLE $B-(x)$

ZPR-6-7 25-GROUP ZONE FISSION FRRCTIONS

\begin{tabular}{|c|c|c|c|c|}
\hline Grous & $\begin{array}{r}\text { Enorgy } \\
\text { Boundory } \\
\text { IeYI }\end{array}$ & $\begin{array}{l}\text { Lethorgy } \\
\text { Hedilh }\end{array}$ & Core & Reflecter \\
\hline $\begin{array}{l}1 \\
2 \\
3 \\
4 \\
5 \\
6 \\
7 \\
8 \\
9 \\
10 \\
11 \\
12 \\
13 \\
14 \\
15 \\
16 \\
17 \\
18 \\
19 \\
20 \\
21 \\
22 \\
23 \\
24 \\
25\end{array}$ & 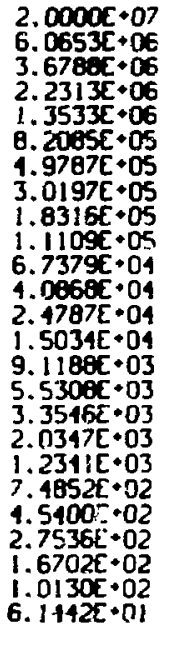 & 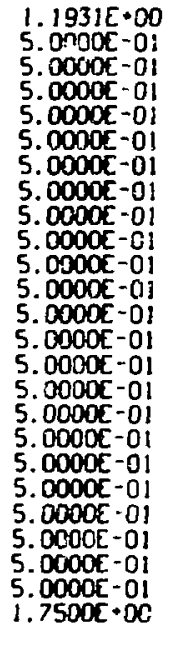 & $\begin{array}{l}2.8774 E-02 \\
1.2141[-01 \\
2.1970 E-01 \\
2.2989 E-01 \\
1.7212 E-01 \\
1.0730 E-01 \\
5.9911 E-02 \\
3.132 E E-02 \\
1.5762 E-02 \\
.7464 E-03 \\
3.7137 E-03 \\
1.7629 E-03 \\
8.3420 E-04 \\
3.9409 E-04 \\
1.8603 E-04 \\
6.7785 E-05 \\
1.1422 E-05 \\
1.9516 E-05 \\
9.2246 E-06 \\
4.3540 E-066 \\
2.0552 E-06 \\
9.7023 E-07 \\
4.5607 E-07 \\
2.1630 E-07 \\
1.9358 E-07 \\
1.0000 E-00\end{array}$ & $\begin{array}{l}2.4792 E-02 \\
1.1293 E-01 \\
2.1334 E-01 \\
2.2843 E-01 \\
1.7576 E-01 \\
1.1236 E-01 \\
6.4203 E-02 \\
3.4157 E-02 \\
1.7590 E-02 \\
8.7130 E-03 \\
4.1107 E-03 \\
1.9174 E-03 \\
8.9485 E-04 \\
4.1817 E-04 \\
1.9571 E-04 \\
5.1734 E-05 \\
1.3057 E-05 \\
2.0225 E-05 \\
9.5094 E-06 \\
4.4755 E-06 \\
2.1056 E-06 \\
9.9077 E-07 \\
4.6646 E-07 \\
2.1967 E-07 \\
1.9652 E-07 \\
1.0000 E-00\end{array}$ \\
\hline
\end{tabular}


TABLE B-LXII

2PR-3-568 70-GROUP TRRASPORT THEORY SPECTRA

\begin{tabular}{|c|c|c|c|c|c|c|c|}
\hline ang & $\begin{array}{r}\text { Einorgy } \\
\text { Boundory } \\
\text { InVI) }\end{array}$ & $\begin{array}{l}\text { Lethorgy } \\
\text { Hidik }\end{array}$ & $\begin{array}{r}\text { Reoctor } \\
\text { Fiescon } \\
\text { Fractions }\end{array}$ & $\begin{array}{r}\text { Control } \\
r \text { lum } \\
\left.\ln / \cos ^{2} / x\right)\end{array}$ & $\begin{array}{r}\text { Centrol } \\
\text { Ad jount } \\
\text { Irhololel } \\
\end{array}$ & $\begin{array}{r}\text { Edgo } \\
\text { flux } \\
\ln / \cos ^{2} / \operatorname{lol} \\
\end{array}$ & $\begin{array}{r}\text { Coge } \\
\text { Ad pont } \\
\text { Ichotalal }\end{array}$ \\
\hline $\begin{array}{l}1 \\
2 \\
3 \\
4 \\
5 \\
6 \\
7 \\
8 \\
3 \\
10 \\
11 \\
12 \\
13 \\
14 \\
15 \\
16 \\
17 \\
18 \\
19 \\
20 \\
21 \\
22 \\
23 \\
21 \\
25 \\
26 \\
27 \\
28 \\
29 \\
30 \\
31 \\
32 \\
33 \\
74 \\
35 \\
36 \\
37 \\
38 \\
39 \\
10 \\
11 \\
12 \\
43 \\
44 \\
45 \\
46 \\
47 \\
48 \\
19 \\
50 \\
51 \\
52 \\
53 \\
54 \\
55 \\
56 \\
57 \\
58 \\
59 \\
60 \\
61 \\
52 \\
53 \\
64 \\
65 \\
56 \\
57 \\
68 \\
59 \\
70\end{array}$ & 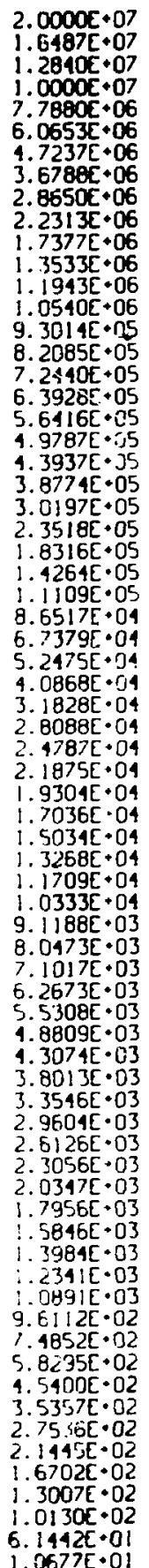 & $\begin{array}{l}1.9315 E-01 \\
2.5000 E-01 \\
2.5000 E-01 \\
2.5000 E-01 \\
2.5000 E-01 \\
2.5000 E-01 \\
2.5000 E-01 \\
2.5000 E-01 \\
2.5000 E-01 \\
2.5000 E-01 \\
2.5000 E-01 \\
1.2500 E-01 \\
1.2500 E-01 \\
1.2500 E-01 \\
1.2500 E-01 \\
1.2500 E-01 \\
1.2500 E-01 \\
1.2500 E-01 \\
1.2500 E-01 \\
1.2500 E-01 \\
1.2500 E-01 \\
2.5000 E-01 \\
2.5000 E-01 \\
2.5000 E-01 \\
2.5000 E-01 \\
2.5000 E-01 \\
2.5000 E-01 \\
2.5000 E-01 \\
2.5000 E-01 \\
2.5000 E-01 \\
2.5000 E-01 \\
1.25 C 0 E-01 \\
1.2500 E-01 \\
1.2500 E-01 \\
1.2500 E-01 \\
1.2500 E-01 \\
1.2500 E-01 \\
1.2500 E-01 \\
1.2500 E-01 \\
1.2497 E-01 \\
1.2503 E-01 \\
1.2500 E-01 \\
1.2500 E-01 \\
1.2500 E-01 \\
1.2500 E-01 \\
1.2500 E-01 \\
1.2500 E-01 \\
1.2500 E-01 \\
1.2500 E-01 \\
1.2500 E-01 \\
1.2500 E-01 \\
1.2500 E-01 \\
1.2500 E-01 \\
1.25001-01 \\
1.2500 E-01 \\
1.2500 E-01 \\
1.2500 E-01 \\
1.2500 E-01 \\
1.2500 E-01 \\
2.5000 E-01 \\
2.5000 E-01 \\
2.5000 E-01 \\
2.5000 E-01 \\
2.5000 E-01 \\
2.5000 E-01 \\
2.5000 E-01 \\
2.5000 E-01 \\
2.5000 E-01 \\
5.0000 E-01 \\
1.7500 E-00\end{array}$ & $\begin{array}{l}7.9707 E-06 \\
1.4767 E-04 \\
1.3255 E-03 \\
6.6068 E-03 \\
2.0776 E-02 \\
4.5663 E-02 \\
7.6102 E-02 \\
1.0256 E-01 \\
1.1753 E-01 \\
1.1907 E-01 \\
1.0990 E-01 \\
1.9415 E-02 \\
4.5213 E-02 \\
1.0830 E-02 \\
3.6501 E-02 \\
3.2347 E-02 \\
2.8389 E-02 \\
2.4301 E-02 \\
2.1521 E-02 \\
1.8556 E-02 \\
1.5919 E-02 \\
2.5229 E-02 \\
1.8197 E-02 \\
1.2994-02 \\
9.1992 E-03 \\
6.4805 E-03 \\
1.5406 E-03 \\
3.1620 E-03 \\
2.1859 E-03 \\
1.5080 E-03 \\
1.0390 E-03 \\
3.9096 E-04 \\
3.2431 E-04 \\
2.6999 E-04 \\
2.2308 E-04 \\
1.8497 E-04 \\
1.5337 E-04 \\
1.2716 E-04 \\
1.0542 E-01 \\
8.7369 E-05 \\
7.2461 E-05 \\
6.0047 E-05 \\
4.9773 E-05 \\
4.1255 E-05 \\
3.4195 E-05 \\
2.8342 E-05 \\
2.3491 E-05 \\
1.947 E-05 \\
1.6138 E-05 \\
1.3375 E-05 \\
1.1086 E-05 \\
9.1885 E-06 \\
7.6159 E-06 \\
6.3122 E-06 \\
5.2318 E-06 \\
1.3362 E-06 \\
3.5942 E-06 \\
2.9790 E-06 \\
2.4693 E-06 \\
3.7432 E-06 \\
2.5717 E-06 \\
1.7669 E-06 \\
1.2140 E-06 \\
8.3414 E-07 \\
5.7315 E-07 \\
3.9380 E-07 \\
2.7060 E-07 \\
1.8594 E-07 \\
2.1558 E-07 \\
1.9294 E-07 \\
1.0000 E-00\end{array}$ & $\begin{array}{l}8.2741 E-07 \\
1.5958 E-05 \\
1.2685-04 \\
7.2390 E-04 \\
2.3207 E-03 \\
5.3614 E-03 \\
8.9501 E-03 \\
1.4292 E-02 \\
2.2640 E-02 \\
2.3874 E-02 \\
2.8803 E-02 \\
1.6139 E-02 \\
1.6456 E-02 \\
1.3937 E-02 \\
1.98 B 2 E-02 \\
2.4277 E-02 \\
2.7134 E-02 \\
3.1247 E-02 \\
3.3563 E-02 \\
2.3063 E-C 2 \\
1.8665 E-02 \\
5.5610 E-02 \\
5.8106 E-02 \\
5.4799 E-02 \\
5.0634 E-02 \\
5.6471 E-02 \\
4.8172 E-02 \\
5.0602 E-02 \\
3.4849 E-02 \\
3.8945 E-02 \\
3.6441 E-02 \\
6.8464 E-03 \\
1.9531 E-02 \\
1.7089 E-02 \\
1.5229 E-02 \\
1.3297 E-02 \\
1.0315 E-02 \\
1.1160 E-02 \\
9.5719 E-03 \\
9.2848 E-03 \\
7.8223 E-03 \\
5.5712 E-03 \\
5.0254 E-03 \\
5.5153 E-03 \\
4.876 ! E-03 \\
4.6682 E-03 \\
3.8593 E-03 \\
3.0305 E-03 \\
2.1894 E-03 \\
1.0056 E-03 \\
3.4055 E-04 \\
1.1688 E-03 \\
2.3925 E-03 \\
3.0297 E-03 \\
3.1196 E-03 \\
2.9262 E-03 \\
2.7042 E-03 \\
1.9203 E-03 \\
1.8921 E-03 \\
2.9405 E-03 \\
2.0910 E-03 \\
1.3053 E-03 \\
7.6764 E-C 4 \\
5.3083 E-04 \\
3.0257 E-04 \\
1.7572 E-04 \\
13940 E-05 \\
1.8945 E-05 \\
2.6033 E-05 \\
1.1835 E-05 \\
1.0000 E+00\end{array}$ & 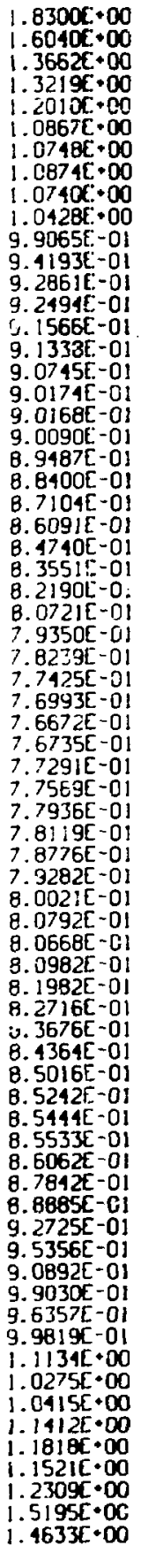 & 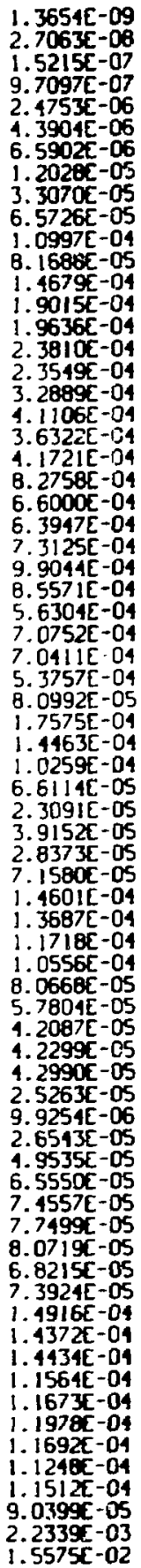 & 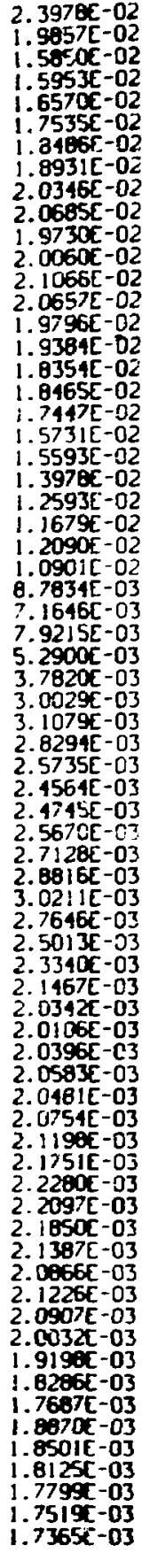 \\
\hline
\end{tabular}


IfQLE B-LXI!I

2PR-3-5G8 70-GROUP ZONE FISSION FRACTIONS

\begin{tabular}{|c|c|c|c|c|}
\hline oug & $\begin{array}{r}\text { Energy } \\
\text { Boundory } \\
\text { Inyl } \\
\end{array}$ & $\begin{array}{l}\text { Lelhorgy } \\
\text { Widilh }\end{array}$ & Core & Rerlector \\
\hline 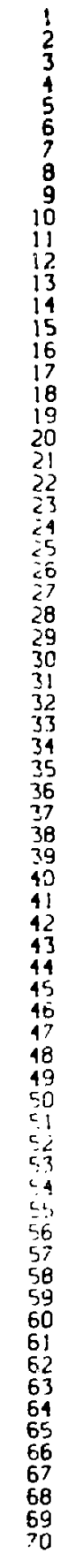 & 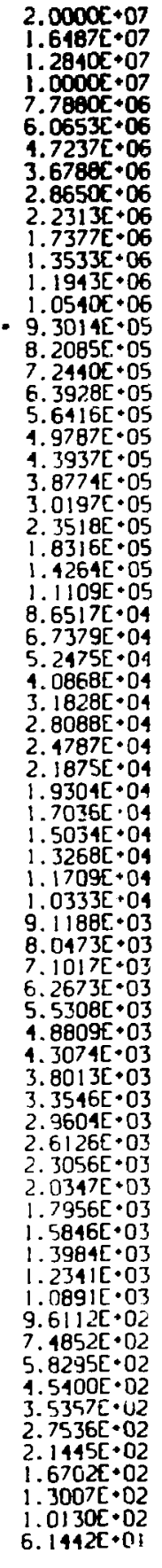 & $\begin{array}{l}1.9315 E-01 \\
2.5000 E-01 \\
2.5000 E-01 \\
2.5000 E-01 \\
2.5000 E-01 \\
2.5000 E-01 \\
2.5000 E-01 \\
2.5000 E-01 \\
2.5000 E-01 \\
2.5000 E-01 \\
2.5000 E-01 \\
1.2500 E-01 \\
1.2500 E-01 \\
1.2500 E-01 \\
1.2500 E-01 \\
1.2500 E-01 \\
1.2500 E-01 \\
1.2500 E-01 \\
1.2500 E-01 \\
1.2500 E-01 \\
1.2500 E-01 \\
2.5000 E-01 \\
2.5000 E-01 \\
2.5000 E-01 \\
2.5000 E-01 \\
2.5000 E-01 \\
2.5000 E-01 \\
2.5000 E-01 \\
2.5000 E-01 \\
2.5000 E-01 \\
2.5000 E-01 \\
1.2500 E-01 \\
1.2500 E-01 \\
1.2500 E-01 \\
1.2500 E-01 \\
1.2500 E-01 \\
1.2500 E-01 \\
1.2500 E-01 \\
1.2500 E \\
1.2497 \\
1.250 \\
1.2503 E\end{array}$ & $\begin{array}{l}7.9707 E-06 \\
1.4767 E-04 \\
1.3255 E-03 \\
6.6068 E-03 \\
2.0776 E-02 \\
4.5663 E-02 \\
7.6102 E-02 \\
1.0256 E-01 \\
1.1753 E-01 \\
1.1907 E-01 \\
1.0990 E-01 \\
4.9415 E-02 \\
4.5213 E-02 \\
4.0830 E-02 \\
3.6501 E-02 \\
3.2347 E-02 \\
2.8389 E-02 \\
2.4801 E-02 \\
2.1521 E-02 \\
1.8556 E-02 \\
1.5919 E-02 \\
2.5229 E-02 \\
1.8197 E-02 \\
1.2994 E-02 \\
9.1992 E-03 \\
6.4805 E-03 \\
4.5406 E-03 \\
3.1620 E-03 \\
2.1859 E-03 \\
1.5080 E-03 \\
1.0390 E-03 \\
3.9096 E-04 \\
3.2431 E-04 \\
2.6899 E-04 \\
2.2308 E-04 \\
1.8497 E-04 \\
1.5337 E-04 \\
1.2716 E-04 \\
1.0542 E-04 \\
8.7365 E-05 \\
7.2461 E-05 \\
6.0047 E-05 \\
1.9773 E-05 \\
4.1255 E-05 \\
3.4195 E-05 \\
2.8342 E-05 \\
2.3491 E-05 \\
1.9471 E-05 \\
1.6138 E-05 \\
1.3375 E-05 \\
1.1086 E-05 \\
9.1865 E-06 \\
7.6159 E-06 \\
6.3122 E-06 \\
5.2318 E-06 \\
1.3362 E-06 \\
3.5942 E-06 \\
2.9790 E-06 \\
2.4693 E-05 \\
3.7432 E-06 \\
2.5717 E-06 \\
1.7669 E-06 \\
1.2140 E-06 \\
8.3414 E-07 \\
5.7315 E-07 \\
3.9380 E-07 \\
2.7060 E-07 \\
1.8594 E-07 \\
2.1558 E-07 \\
1.9294 E-07 \\
1.0000 E-00\end{array}$ & 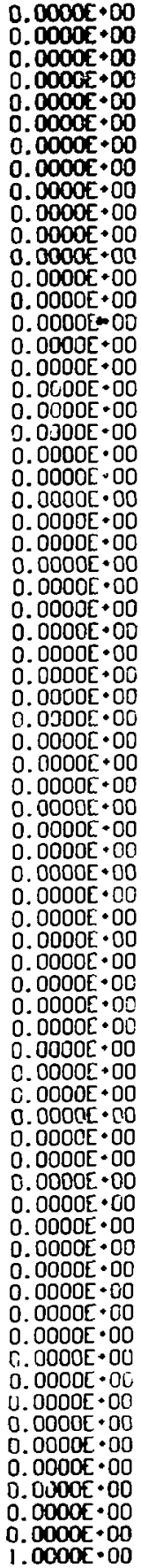 \\
\hline
\end{tabular}


TFBLE B-LXIV

ZPR-3-568 25-GROUP IRAWSPORT THEORY SPECTRA

\begin{tabular}{|c|c|}
\hline Equ & $\begin{array}{r}\text { Energy } \\
\text { Boundory } \\
\text { Invi }\end{array}$ \\
\hline $\begin{array}{l}1 \\
2 \\
3 \\
4 \\
5 \\
6 \\
7 \\
8 \\
9 \\
10 \\
11 \\
12 \\
13 \\
14 \\
15 \\
16 \\
17 \\
18 \\
19 \\
20 \\
21 \\
22 \\
23 \\
24 \\
25\end{array}$ & 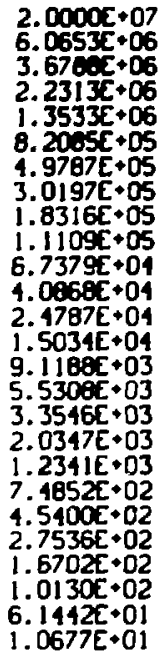 \\
\hline
\end{tabular}

\begin{tabular}{|c|c|}
\hline $\begin{array}{r}\text { Lethorgy } \\
\text { Hidih }\end{array}$ & $\begin{array}{r}\text { Reoctor } \\
\text { Fisuion } \\
\text { Eroction }\end{array}$ \\
\hline 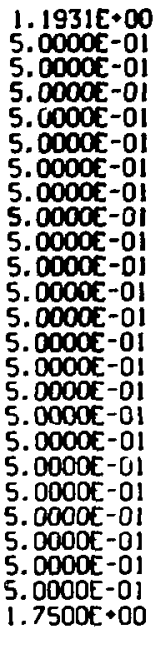 & $\begin{array}{l}2.8064 E-02 \\
1.2176 E-01 \\
2.2006 E-01 \\
2.2697 E-01 \\
1.7196 E-01 \\
1.0706 E-01 \\
5.9704 E-02 \\
3.1191 E-02 \\
1.5680 E-02 \\
7.7027 E-03 \\
3.6939 E-03 \\
1.7543 E-03 \\
8.3041 E-04 \\
3.9241 E-04 \\
1.8527 E-04 \\
8.741 E-05 \\
4.1266 E-05 \\
1.9474 E-05 \\
9.1915 E-06 \\
1.3386 E-06 \\
2.0481 E-06 \\
9.6695 E-07 \\
1.5651 E-07 \\
2.1558 E-07 \\
1.0204 E-07\end{array}$ \\
\hline
\end{tabular}

$\begin{array}{r}\text { Conlral } \\ \text { Llux } \\ \text { nn/en } 2 / 21\end{array}$
$3.1883 E-03$
$1.4312 E-02$
$3.6931 E-02$
$5.2677 E-02$
$6.6413 E-02$
$1.1625 E-01$
$9.7337 E-02$
$1.1291 E-01$
$1.0710 E-01$
$9.6774 E-02$
$7.3793 E-02$
$6.2919 E-02$
$5.5931 E-02$
$3.7939 E-02$
$2.0968 E-02$
$1.3747 E-02$
$1.9074 E-03$
$1.1790 E-02$
$6.7529 E-03$
$3.3963 E-03$
$1.2985 E-03$
$1.7828 E-01$
$1.4288 E-01$
$2.6033 E-05$
$1.1835 E-05$
$1.0000 E+00$

\begin{tabular}{r} 
Centrol \\
Rdjolnt \\
Irhotolel \\
\hline $2.7025 E+00$ \\
$7.9765 E-01$ \\
$7.9760 E-01$ \\
$7.5041 E-01$ \\
$1.3695 E+00$ \\
$1.3374 E+00$ \\
$9.8891 E-01$ \\
$6.3914 E-01$ \\
$6.2104 E-01$ \\
$6.0119 E-01$ \\
$5.8155 E-01$ \\
$8.5279 E-01$ \\
$1.1423 E+00$ \\
$1.1669 E+00$ \\
$1.1972 E+00$ \\
$1.2391 E+00$ \\
$1.2631 E+00$ \\
$1.3463 E+00$ \\
$1.0565 E+00$ \\
$7.7925 E-01$ \\
$7.6352 E-01$ \\
$8.5725 E-01$ \\
$6.7939 E-01$ \\
$5.6074 E-01$ \\
$5.3998 E-01$
\end{tabular}

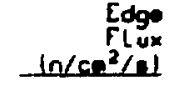

3. $6268 \mathrm{C}-06$

1. $0991 E-05$

4. $5098 \mathrm{c}-05$

$1.7569 c-04$

6. $1499 E-04$

1. $2136 \mathrm{E}-03$

1. 6080c-03

$1.2995 E-03$

$1.7217 \mathrm{E}-03$

$1.41885-03$

1.4116E-03

7.9432E-04

3. 354 3E-04

$2.8511 E-04$

4. 4028[-04

$1.85 ! 85-04$

1. $1127 \mathrm{E}-04$

2.9832E-04

2. $9129 \mathrm{c}-04$

2. $8806 E-04$

2. $3236 \mathrm{E}-04$

2. 3670 -04

2. 2760C-04

9.03995-05

2. 2339ז-03

1. $5575 \mathrm{E}-02$
Ed jount lrholn $(x)$

3. $1027 t-02$ 1. 3293t-02

$1.4454 E-02$

$1.4914 t-02$

$3.0105 t-02$

2.71795-02

1.6718r-02

b. $9568 \mathrm{E}-03$

0. $4843 E-03$

$5.8853 \mathrm{E}-03$

4. $8754 \mathrm{E}-03$

3. $6507[-03$

3. 81 $35[-03$

4. $1267 \mathrm{E}-93$

$3.5968 \mathrm{C}-03$

3. $0049 t-03$

3. $10665-03$

3. $2332 \mathrm{E}-03$

2. $3248 \mathrm{C}-03$

1. 4477E- 03

1. $3275[-03$

1. 3791E-03

1. 3257E-03

6. $1649 \mathrm{C}-04$

$6.4081 E-04$

IABLE B-LXV

ZPR-3-56B 25-GROUP ZONE FISSION FRACTIONS

\begin{tabular}{|c|c|c|c|c|}
\hline enc & $\begin{array}{r}\text { Energy } \\
\text { Boundory } \\
\text { IeYI] }\end{array}$ & $\begin{array}{r}\text { Lethorgy } \\
\text { Hidith }\end{array}$ & Core & Rerlects \\
\hline $\begin{array}{r}1 \\
2 \\
3 \\
1 \\
5 \\
6 \\
7 \\
8 \\
9 \\
10 \\
11 \\
12 \\
13 \\
14 \\
15 \\
16 \\
17 \\
18 \\
19\end{array}$ & $\begin{array}{l}2.0000 E+07 \\
6.0653 E+06 \\
3.6786 E+06 \\
2.2313 E+06 \\
1.3533 E+06 \\
8.2085 E+05 \\
1.9787 E+05 \\
3.0197 E+05 \\
1.8316 E+05 \\
1.1109 E+05 \\
6.1379 \mathrm{C} \\
4.0868+04 \\
2.4787 E+04 \\
1.5034 E+04 \\
9.1186 E+03 \\
5.5308 E+03 \\
3.3546 E+03 \\
2.0347 E+03 \\
1.2341 E+03 \\
7.4852 E+02 \\
4.5400 E+02 \\
2.7536 E+02 \\
1.6702 E+02 \\
1.0130 E+02 \\
6.1442 E+01\end{array}$ & $\begin{array}{r}1.1931 E+00 \\
5.0000 E-01 \\
5.0000 E-01 \\
5.0000 E-01 \\
5.0000 E-01 \\
5.0000 E-01 \\
5.0000 E-01 \\
5.0000 E-01 \\
5.0000 E-01 \\
5.0000 E-01 \\
5.0000 E-01 \\
5.0000 E-01 \\
5.0000 E-01 \\
5.0000 E-01 \\
5.0000 E-01 \\
5.0000 E-01 \\
5.0000 E-01 \\
5.0000 E-01 \\
5.0000 E-01 \\
5.0000 E-01 \\
5.0000 E-01 \\
5.0000-01 \\
5.0000 E-01 \\
5.0000 E-01 \\
1.7500 E-01\end{array}$ & 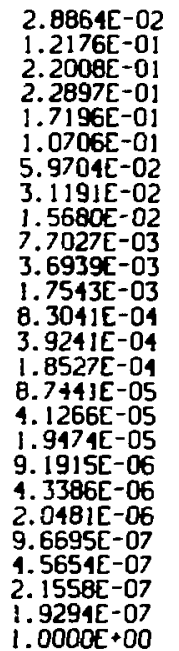 & $\begin{array}{l}0.0000 E+00 \\
0.0000 E+00 \\
0.0000 E+000 \\
0.0000 E+00 \\
0.0000 E+00 \\
0.0000 E+00 \\
0.0000 E+00 \\
0.0000 E+00 \\
0.0000 E+00 \\
0.0000 E+00 \\
0.0000 E+00 \\
0.0000 E+00 \\
0.0000 E+00 \\
0.0000 E+00 \\
0.0000 E+00 \\
0.0000 E+00 \\
0.0000 E+00 \\
0.0000 E * 00 \\
0.0000 E+00 \\
0.0000 E+00 \\
0.0000 E+00 \\
0.0000 E+00 \\
0.0000 E+00 \\
0.0000 E+00 \\
0.0000 E+00 \\
1.0000 E+00\end{array}$ \\
\hline
\end{tabular}


THuL B-LXYI

2PR-6-6A 70-GNOLP IAMUSPOAT THEORY SPECTRA

\begin{tabular}{|c|c|c|c|c|c|c|}
\hline 010 & $\begin{array}{r}\text { Energy } \\
\text { Boundory } \\
\text { IfyI }\end{array}$ & $\begin{array}{l}\text { Lethorgy } \\
\text { Hidilh }\end{array}$ & $\begin{array}{l}\text { Reoclor } \\
\text { fiseion } \\
\text { frectiven }\end{array}$ & $\begin{array}{r}\text { Cenlrol } \\
r l u x \\
\log \left(\operatorname{con}^{2} / x\right)\end{array}$ & $\begin{array}{r}\text { Control } \\
\text { Adjoint } \\
\text { (rhotal }\end{array}$ & $\begin{array}{r}\frac{E}{F} \\
\ln \left\langle e e^{2}\right.\end{array}$ \\
\hline $\begin{array}{l}1 \\
2 \\
3 \\
1 \\
5 \\
6 \\
7 \\
6 \\
9 \\
10 \\
11 \\
12 \\
13 \\
14 \\
15 \\
16 \\
17 \\
18 \\
19 \\
20 \\
21 \\
22 \\
23 \\
24 \\
25 \\
26 \\
27 \\
28 \\
29 \\
30 \\
31 \\
32 \\
33 \\
34 \\
35 \\
36 \\
37 \\
38 \\
39 \\
40 \\
41 \\
42 \\
43 \\
44 \\
45 \\
46 \\
47 \\
48 \\
49 \\
50 \\
51 \\
52 \\
53 \\
54 \\
55 \\
56 \\
57 \\
58 \\
59 \\
60 \\
61 \\
62 \\
63 \\
64 \\
65 \\
66 \\
67 \\
68 \\
69 \\
70\end{array}$ & 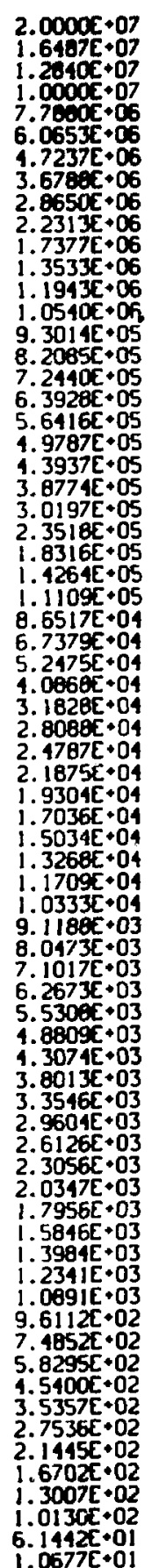 & 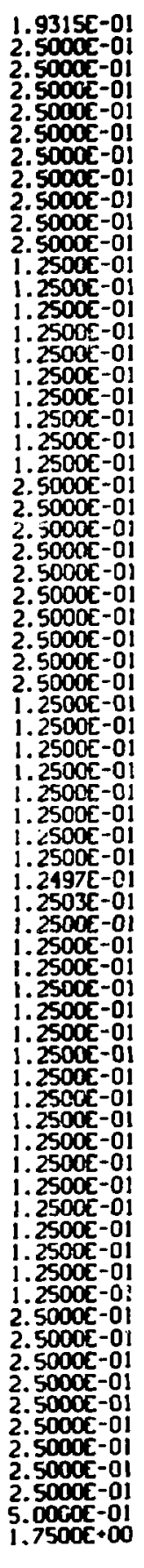 & 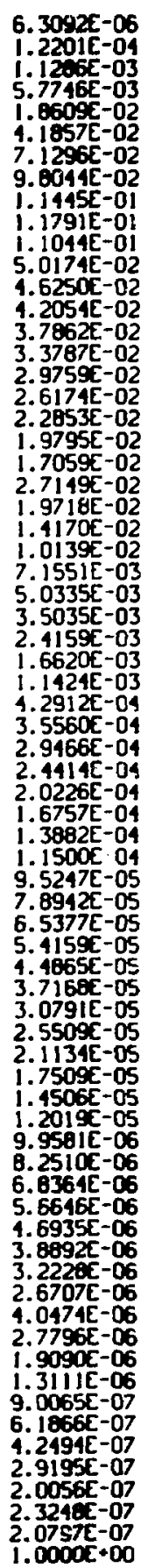 & 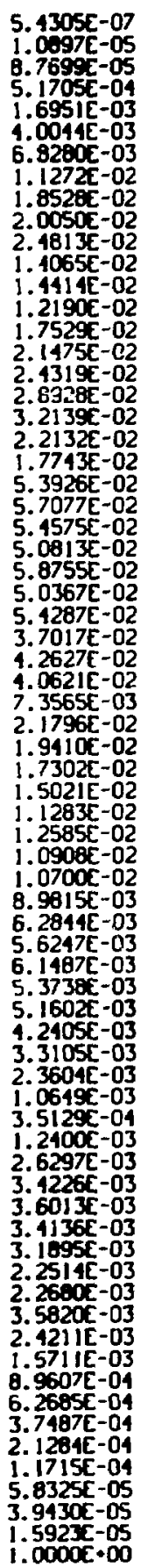 & 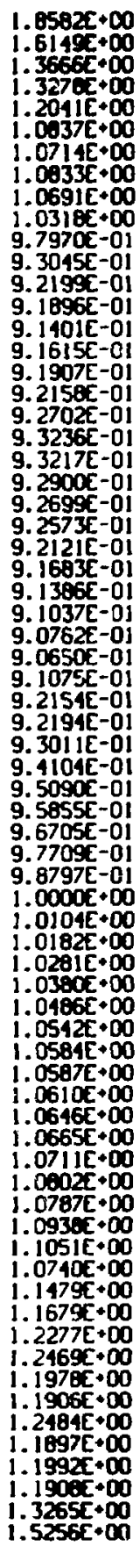 & 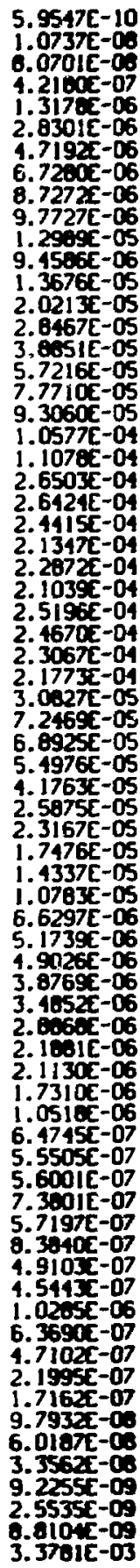 \\
\hline
\end{tabular}

$\underset{\text { ndjoint }}{\cos }$

3. 3155E-02 $2.719 z-02$ 2. $1600 \mathrm{c}-02$ 1.013 $c-02$ 1. $50 \cdot 2-02$ 1. $143-02$ $1.00402-02$

$9.234:-03$ $0.6503 \mathrm{x}-03$ $7.773-2-03$ $6.59005-03$ 4. $6555 \mathrm{E}-03$ 4. $63552-03$ 5.0037 -03

5. 3537t-03 5.5576z-03 5. $40.915-03$ 4.9977t-03 4. $1035[-03$ 3. 0.00:-03 3. 351 다 -03 2.9375 $\mathrm{E}-03$ 2. $2221 \mathrm{E}-03$ 1. 6544 -03 $1.3261 \mathrm{E}-03$

1.1145t-03 $9.1735 \mathrm{~L}-04$ $7.9205-04$ 7.0105-04 $5.071 \mathrm{C}-0$ 1.0597t-04 1.0227E-04 4. $1200=04$ 4. $1202 x-04$ 3.7105e-04 3.354IE-04 3.05075-04 3. $0565 \mathrm{x}-0$ 2.9764E-04 $2.9713 \mathrm{~F}-04$ 2.919:-04 $2.823 \mathrm{~s}-04$ 2. $0079-04$ 2.927 $=-0$ 2. 900:-04 3.0916:-04 $3.1533 \mathrm{x}-04$ 3. $120=0$ 3. $20.5-04$ 3. $353 x-01$ 3.8157-04 4.6775:-04 5.0005-04 5.6203:-0 5.694 $2-04$ 5.6373z-04 6. $0763 \mathrm{c}-0$ 1. $9935 \mathrm{c}-04$ 5. $07 \cdot 0 \mathrm{c}-0$ $6.2353 \mathrm{z}-04$ 6. $62525-0$. 7.579.5-04 6. 600 7.0331E-04 7. 3734-04 7.3543-0 5.eAsIE-01 1. - 2251E-03 2.010 $=-03$ 
TFELE B-LXVII

2PR-6-6H 70-GROUP ZONE FISSION FRACTIGNS

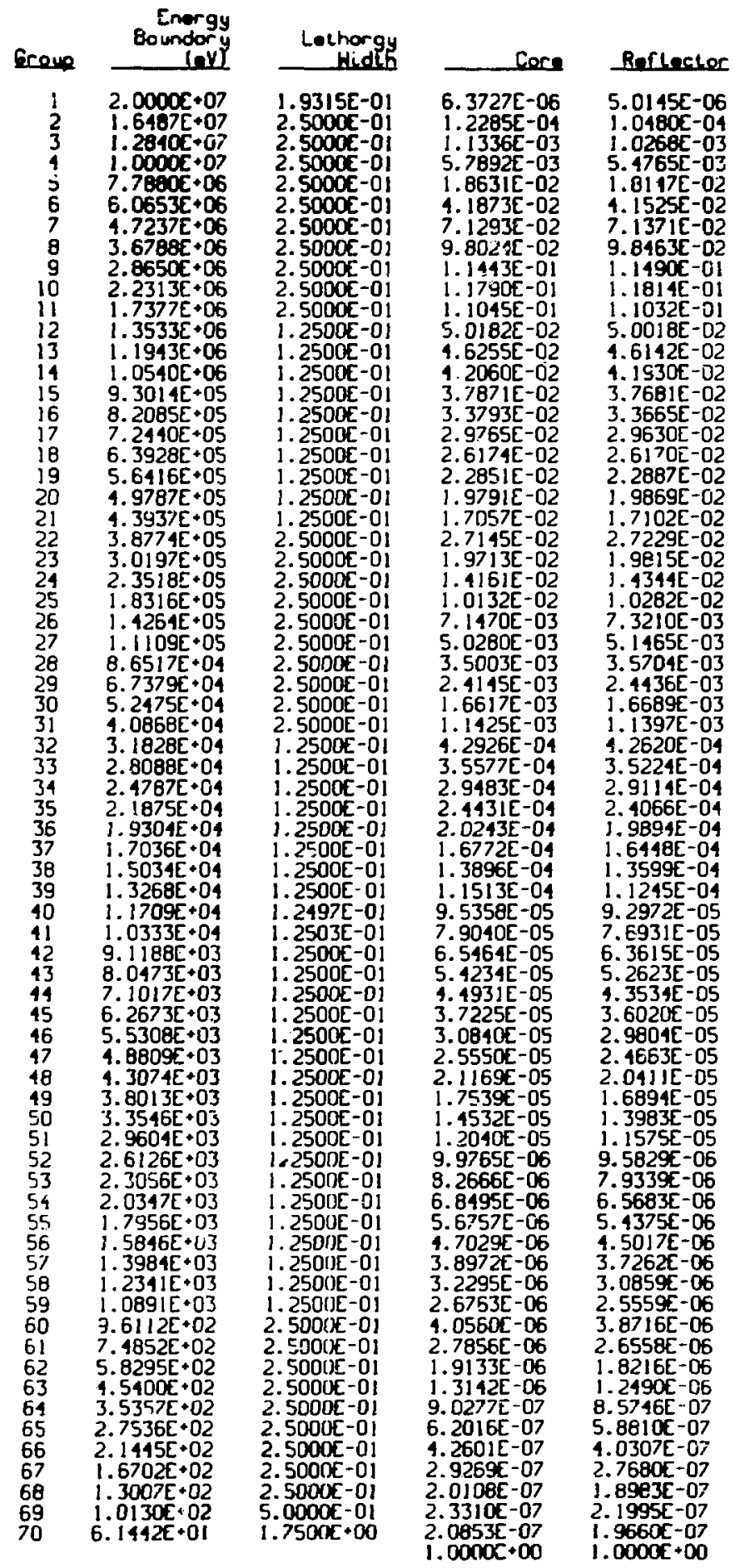


TFELE B-LXVIII

2PR-6-6A 25-GROUP TRANSPORT THEORY SPECTRA

\begin{tabular}{|c|c|}
\hline Scons & $\begin{array}{r}\text { Energy } \\
\text { Boundory } \\
\text { (fy) }\end{array}$ \\
\hline $\begin{array}{c}1 \\
2 \\
3 \\
1 \\
5 \\
6 \\
7 \\
8 \\
9 \\
10 \\
11 \\
12 \\
13 \\
14 \\
15 \\
16 \\
17 \\
16 \\
19 \\
20 \\
21 \\
22 \\
23 \\
24 \\
25\end{array}$ & $\begin{array}{l}2.0000 E+07 \\
6.065 z E+06 \\
3.670 E+05 \\
2.2313 E+06 \\
1.3533 E+05 \\
8.2085 E+05 \\
4.9787 E+05 \\
3.0197 E+05 \\
1.6316 E+05 \\
1.1109 E+05 \\
6.7379 E+04 \\
1.0868 E+04 \\
2.1767 E+04 \\
1.5034 E+04 \\
9.1188 E+03 \\
5.5308 E+03 \\
3.3546 E+03 \\
2.0347 E+03 \\
1.2341 E+03 \\
7.4852 E+02 \\
1.5400 E+02 \\
2.7536 E+02 \\
1.6702 E+02 \\
1.0130 E+02 \\
6.1442 E+01 \\
1.0677 E+01\end{array}$ \\
\hline
\end{tabular}

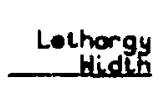

$1.1931 E+00$

5. $0000 \mathrm{c}-01$

5. $0000 \mathrm{C}-01$

5. 000CE-01

5. $00005-01$

5. G000E-01

5.0000E-01

5.0000E-01

5. 0000 -01

$5.0000 \varepsilon-01$

5. $0000 \mathrm{c}-01$

5. $0000 \mathrm{E}-01$

5. $0000 \mathrm{E}-01$

5. 000OE-01

5. $0000 E-01$

5. $0000 \mathrm{E}-01$

5. $0000 E-01$

5. $0000 \mathrm{C}-01$

5.000\%L-0

5. $0000 \mathrm{c}-0$

$5.0000 E-01$

5. $0000 E-01$

5. $00005-01$

5. $0000 \mathrm{E}-01$

1. $7500 \mathrm{c}+00$

\begin{tabular}{r} 
Reoclor \\
Fiseion \\
froction \\
\hline $2.5640 c-02$ \\
$1.1315 E-01$ \\
$2.1250 E-01$ \\
$2.2035 E-01$ \\
$1.7634 E-01$ \\
$1.1257 E-01$ \\
$6.1003 E-02$ \\
$3.3887 E-02$ \\
$1.7294 E-02$ \\
$8.5370 E-03$ \\
$4.0779 E-03$ \\
$1.9271 E-03$ \\
$9.0863 E-04$ \\
$4.2801 E-04$ \\
$2.0157 E-04$ \\
$9.4942 E-05$ \\
$4.4731 E-05$ \\
$2.1084 E-05$ \\
$9.9409 E-06$ \\
$4.6886 E-06$ \\
$2.2118 E-06$ \\
$1.0436 E-06$ \\
$4.9250 E-07$ \\
$2.3248 E-07$ \\
$2.0797 E-07$ \\
$1.0000 E+00$
\end{tabular}

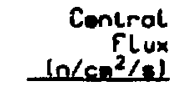

2. $3113 \mathrm{E}-03$

1. $0632 z-02$

2. $9000 \mathrm{c}-02$

4. $4863 x-02$

5.81995-02

1.0685e-01

9. 38025-02

$1.1165 \varepsilon-01$

1.0957E-ci

1.0465E-01

$7.9645 \varepsilon-02$

6.9774E-02

6. 3016E-02

4. $3174 \mathrm{E}-02$

2. 3432E-02

1. 5072E-02

5. $2859 \mathrm{E}-03$

$1.3627 \mathrm{E}-02$

8. $1014 E-03$

$3.9921 \mathrm{E}-03$

$1.5229 \mathrm{E}-03$

$5.8772 E-04$

1.7548E-04

3.9130E-05

1. $5923 E-05$

1. $0000 \mathrm{C}+00$

Central
Adjoint
rrhotolel

$\begin{array}{r}\text { Edge } \\ \text { flux } \\ \ln / a n^{2} / n l \\ \hline\end{array}$

$1.8316 E-05$

$7.5493 \mathrm{E}-06$

1.5455t-05

$2.2762 \mathrm{E}-05$

$7.1814 \mathrm{E}-05$

2.6684E-04

4. $3158 \mathrm{c}-04$

5.0839c-04

4.4219E-04

$4.6236 \mathrm{t}-04$

4.7737E-04

$3.2103 E-04$

1.9154E-04

$6.5763 \mathrm{E}-05$

2. $.583 E-05$

$1.0673 \mathrm{E}-05$

3. $9853 E-06$

2.7084E-06

$1.9740 \mathrm{~F}-26$

1. 1079E-06

3.9158c-07

1.5812E-07

$4.2788 E-C B$

2. $55355-09$

8. 8104E-09

3. $3781 \mathrm{E}-03$
Edge
Ad pont

Irho/n/sI

1. 2422 -02

$7.680 .2-03$

6. S6E $\mathrm{E}-03$

$5.2756 E-05$

7. 2495E-03

$7.5097 \mathrm{E}-03$

3. $7276 \mathrm{E}-03$

$1.4274 E-03$

0.9537e-04

6. 30$\} 1 \mathrm{E}-04$

4.7370E-04

$4.7758 \mathrm{C}-\mathrm{G4}$

$5.2343 E-04$

4. 3795E- 04

$4.2417 \mathrm{E}-04$

$4.6513 \mathrm{C}-04$

6. $1911 \mathrm{E}-04$

8. $4612 \mathrm{E}-04$

$6.2831 E-04$

$5.2172 E-G 4$

5.0424E-04

$5.27935-04$

4. $8515 k-04$

5.2377E-04

$7.5253 E-04$

TABLE 8-LXIX

2PR-6-6A 25-GROUP ZONE FISSION FRRCTIONS

\begin{tabular}{|c|c|c|c|c|}
\hline cones & $\begin{array}{r}\text { Energy } \\
\text { Boundory } \\
\text { (eyI) }\end{array}$ & $\begin{array}{r}\text { Lethorgy } \\
\text { Hidih }\end{array}$ & Core & Riflector \\
\hline $\begin{array}{r}1 \\
2 \\
3 \\
4 \\
5 \\
6 \\
7 \\
8 \\
9 \\
10 \\
11 \\
12 \\
13 \\
14 \\
15 \\
16 \\
17 \\
18 \\
19 \\
20 \\
21 \\
22 \\
23 \\
24 \\
25\end{array}$ & 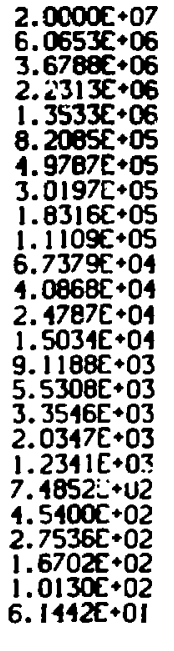 & 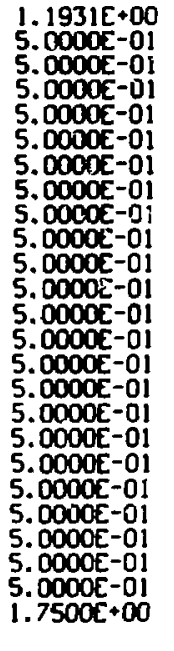 & $\begin{array}{l}2.5683 E-02 \\
1.1317 E-01 \\
2.1246 \varepsilon-01 \\
2.2835 E-01 \\
1.7637 E-01 \\
1.1258 E-01 \\
6.3994 E-02 \\
3.3874 E-02 \\
1.7279 E-02 \\
8.5282 E-03 \\
4.0762 E-03 \\
1.9276 E-03 \\
9.0929 E-04 \\
4.2849 E-04 \\
2.0185 E-04 \\
9.5098 E-05 \\
4.4915 E-05 \\
2.1125 E-05 \\
9.9618 E-06 \\
4.6989 E-06 \\
2.21698-06 \\
1.0462 E-06 \\
4.9377 E-07 \\
2.3310 \varepsilon-07 \\
2.0853 E-07 \\
1.0000 E+00\end{array}$ & $\begin{array}{l}2.4761 E-02 \\
1.1290 E-01 \\
2.1337 E-01 \\
2.2816 E-01 \\
1.7577 E-01 \\
1.1235 E-01 \\
6.4200 E-02 \\
3.4150 E-02 \\
1.7603 E-02 \\
8.7168 E-03 \\
4.1125 E-03 \\
1.9182 E-03 \\
8.9522 E-04 \\
4.1835 E-04 \\
1.9579 E-04 \\
9.1772 E-05 \\
4.3076 E-05 \\
2.0234 E-05 \\
9.5134 E-06 \\
4.4774 E-06 \\
2.1065 E-05 \\
9.9117 E-07 \\
4.6664 E-07 \\
2.1995 E-07 \\
1.9660 E-07 \\
1.0000 E+00\end{array}$ \\
\hline
\end{tabular}


TABLE B-LXX

SNEFK-7A 70-GROUP TRFWSPORT IHECRY SPECTRA

\begin{tabular}{|c|c|c|c|c|c|c|c|}
\hline coup & $\begin{array}{r}\text { Enorgy } \\
\text { Boundary } \\
\text { [ov] }\end{array}$ & $\begin{array}{l}\text { Lethorgy } \\
\text { WidlLh }\end{array}$ & $\begin{array}{r}\text { Reoctor } \\
\text { Fiseion } \\
\text { Froction }\end{array}$ & $\begin{array}{r}\text { Centrol } \\
F L u x \\
\text { In/an } 2 / x \mid \\
\end{array}$ & $\begin{array}{r}\text { Centrol } \\
\text { fid joint } \\
\text { holnol }\end{array}$ & $\begin{array}{r}E d g= \\
F \operatorname{lox} \\
\text { lolat } 2 / 1) \\
\end{array}$ & $\begin{array}{l}\text { and } \\
\text { irhol }\end{array}$ \\
\hline $\begin{array}{l}1 \\
2 \\
3 \\
4 \\
5 \\
6 \\
7 \\
8 \\
9 \\
10 \\
11 \\
12 \\
13 \\
11 \\
15 \\
16 \\
17 \\
18 \\
19 \\
20 \\
21 \\
22 \\
23 \\
24 \\
25 \\
26 \\
27 \\
28 \\
29 \\
30 \\
31 \\
32 \\
33 \\
34 \\
35 \\
36 \\
37 \\
38 \\
39 \\
40 \\
41 \\
12 \\
43 \\
44 \\
45 \\
46 \\
47 \\
48 \\
49 \\
50 \\
51 \\
52 \\
53 \\
54 \\
55 \\
56 \\
57 \\
58 \\
59 \\
60 \\
61 \\
62 \\
63 \\
64 \\
65 \\
66 \\
67 \\
68 \\
69 \\
70\end{array}$ & $\begin{array}{l}2.0000 E+07 \\
1.6187 E+07 \\
1.2840 E+07 \\
1.0000 E+07 \\
7.7880 E+06 \\
6.0553 E+06 \\
1.7237 E+06 \\
3.6788 E+06 \\
2.8650 E+06 \\
2.2313 E+06 \\
1.7377 E+06 \\
1.3533 E+06 \\
1.1943 E+06 \\
1.0540 E+06 \\
9.3014 E+05 \\
8.2085 E+05 \\
7.2410 E+05 \\
6.3928 E+05 \\
5.6416 E+05 \\
4.9787 E+05 \\
1.3937 E+05 \\
3.8774 E+05 \\
3.0197 E+05 \\
2.3518 E+05 \\
1.8316 E+05 \\
1.4261 E+05 \\
1.1109 E+05 \\
8.6517 E+04 \\
6.7379 E+04 \\
5.2175 E+04 \\
1.0868 E+04 \\
3.1828 E+04 \\
2.8098 E+01 \\
2.4787 E+04 \\
2.1875 E+04 \\
1.9304 E+04 \\
1.7036 E+04 \\
1.5034 E+04 \\
1.3268 E+04 \\
1.1709 E+04 \\
1.0333 E+04 \\
9.1188 E+03 \\
8.0473 E+03 \\
7.1017 E+03 \\
6.2673 E+03 \\
5.5308 E+03 \\
4.8809 E+03 \\
4.3074 E+03 \\
3.8013 E+03 \\
3.354 E E+03 \\
2.96 n 4 E+03 \\
2.6126 E+03 \\
2.30561+03 \\
2.0347 E+03 \\
1.7956 E+03 \\
1.5846 E+03 \\
1.3984 E+03 \\
1.2341 E+03 \\
1.0891 E+03 \\
9.6112 E+02 \\
7.1852 E+02 \\
5.8295 E+02 \\
4.5400 E+02 \\
3.5357 E+02 \\
2.7536 E+02 \\
2.1445 E+02 \\
1.6702 E+02 \\
1.3007 E+02 \\
1.0130 E+02 \\
6.1442 E+01\end{array}$ & 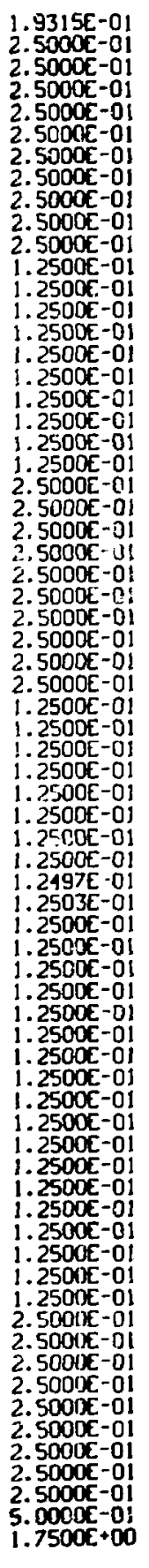 & 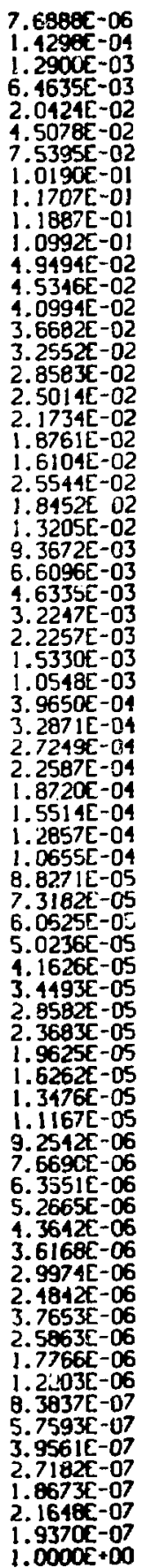 & 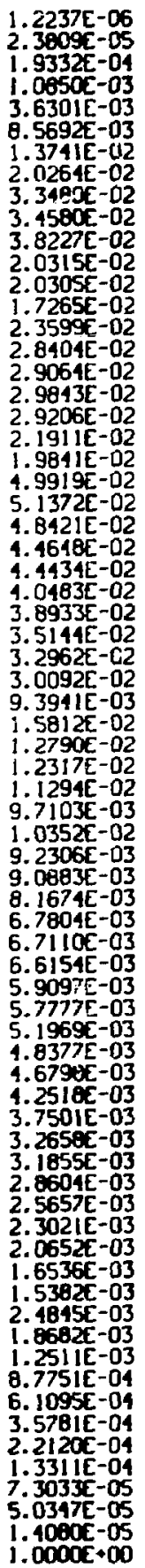 & 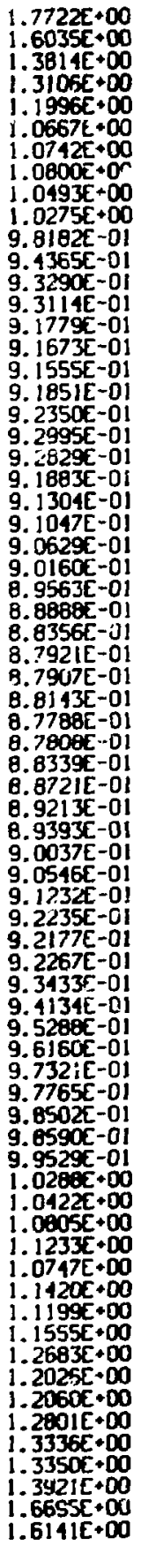 & 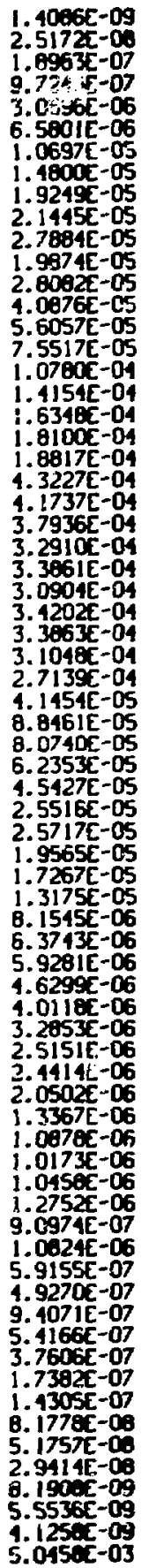 & $\begin{array}{l}02 \\
02 \\
02 \\
02 \\
02 \\
02 \\
02 \\
02 \\
02 \\
03 \\
03 \\
03 \\
03 \\
03 \\
03 \\
03 \\
03 \\
03 \\
03 \\
03 \\
03 \\
03 \\
03 \\
03 \\
03 \\
03 \\
04 \\
04 \\
03 \\
03 \\
04 \\
03 \\
04 \\
04 \\
04 \\
04 \\
04 \\
03 \\
03 \\
03 \\
03 \\
03 \\
03 \\
03 \\
03 \\
04 \\
04 \\
04 \\
04 \\
04 \\
04 \\
04 \\
04 \\
04 \\
04 \\
04 \\
04 \\
04 \\
04 \\
04 \\
04 \\
04 \\
04\end{array}$ \\
\hline
\end{tabular}


TFELE B-LXX]

SAEAK-7A 70-GAOUP 2ONE FISSION FRACTIONS

\begin{tabular}{|c|c|c|c|c|}
\hline 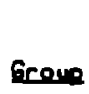 & $\begin{array}{r}\text { Enory } \\
\text { Boundo } \\
\end{array}$ & $\begin{array}{c}\text { Lethor } \\
\text { Llidt }\end{array}$ & Cor & Reflector \\
\hline 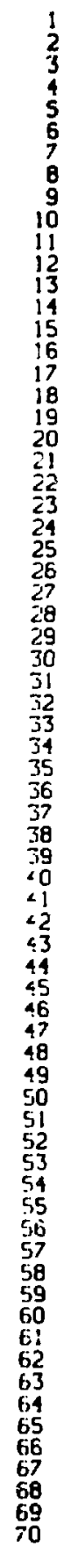 & 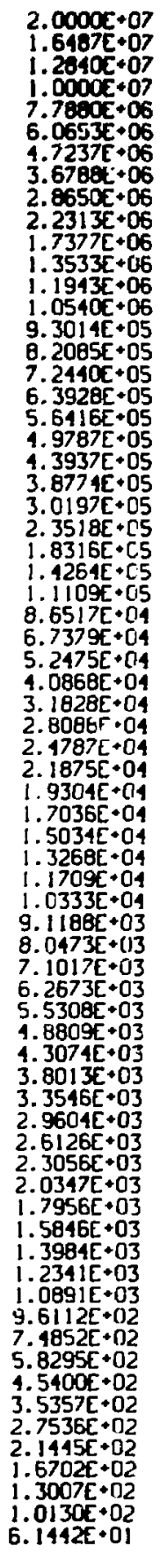 & 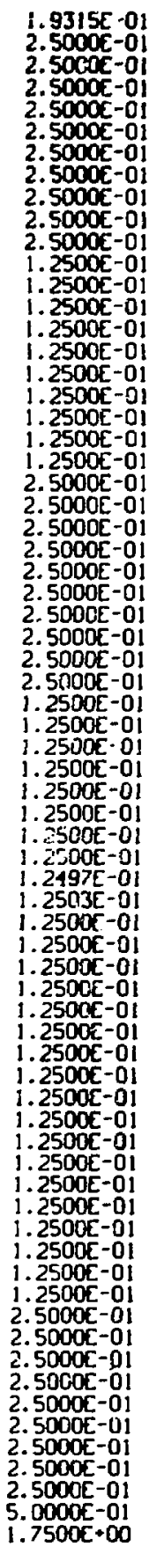 & 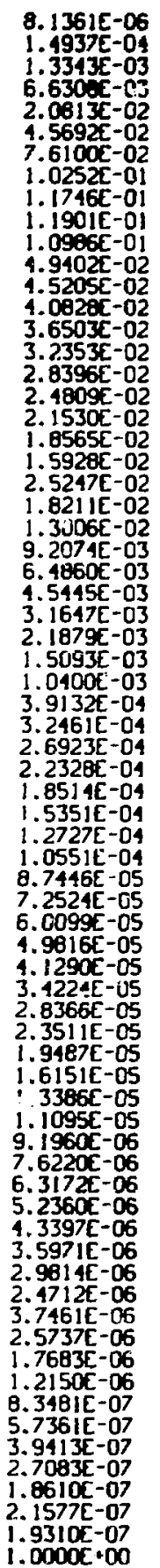 & 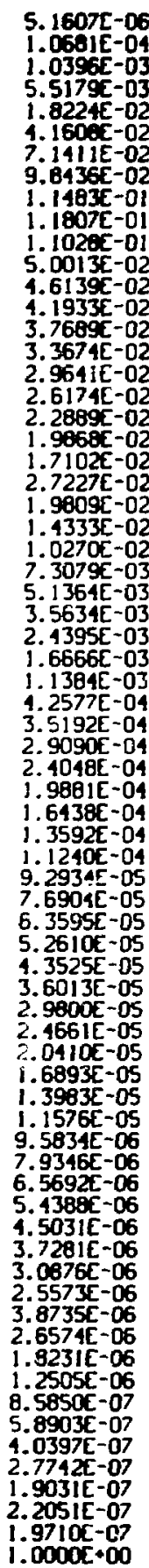 \\
\hline
\end{tabular}


TRBLE $\theta-\lfloor\times \times \mid 1$

SAERK-7A 25-GROUP TRPNSPORT THEORY SPECTRA

\begin{tabular}{|c|c|}
\hline Crom & $\begin{array}{r}\text { Energy } \\
\text { Boundory } \\
\text { CeVI }\end{array}$ \\
\hline $\begin{array}{r}1 \\
2 \\
3 \\
4 \\
5 \\
6 \\
7 \\
6 \\
9 \\
10 \\
11 \\
12 \\
13 \\
14 \\
15 \\
16 \\
17 \\
18 \\
19 \\
20 \\
21 \\
22 \\
23 \\
24 \\
25\end{array}$ & $\begin{array}{l}2.0000 E+07 \\
6.0553 E+05 \\
3.670 E+06 \\
2.2313 E+06 \\
1.3533 E+06 \\
8.2005 E+05 \\
4.9767 E+05 \\
3.0197 E+05 \\
1.0316 E+05 \\
1.1109 E+05 \\
6.7379 E+04 \\
1.0868 E+04 \\
2.4787 E+04 \\
1.5034 E+04 \\
9.1186 E+03 \\
5.5300 E+03 \\
3.3546 E+03 \\
2.0347 E+03 \\
1.2341 E+03 \\
7.4852 E+02 \\
4.5400 E+02 \\
2.7536 E+02 \\
1.6702 E+02 \\
1.0130 E+02 \\
6.1442 E+01 \\
1.0677 E+01\end{array}$ \\
\hline
\end{tabular}

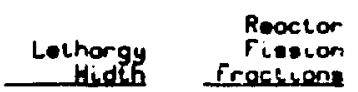

1. $1931 \mathrm{E} \cdot 00$

5. $0000 \mathrm{E}-01$

5. $0000 \mathrm{C}-01$

5.0000E-01

$5.00005-01$

5. $0000 \mathrm{z}-01$

5. $0000 \mathrm{c}-01$

5. $0000 \mathrm{c}-01$

5. D000E-01

5. $0000 \mathrm{C}-01$

5. $0000 \mathrm{E}-01$

5. $0000 \mathrm{C}-01$

5. $0000 \mathrm{~T}-01$

5. $0000 \mathrm{E}-01$

5. $0000 \mathrm{C}-01$

5. $0000 \mathrm{C}-01$

5.0000E-01

5. $0000 \mathrm{C}-01$

5. 0000E-01

5. $0000 t-01$

5. $0000[-0$

5. $0000 E-01$

5. $0000 \mathrm{E}-0$

$5.0000 E-01$
2. $03285-02$ 1. $2047 \mathrm{E}-01$ 2. 1897E-01 $2.20795-01$ $1.7252 x-01$ 1.07805 -01 $0.0410 \mathrm{~L}-02$ 3. $1657 \mathrm{E}-02$ 7. $0581 \mathrm{E}-03$ 3. $7587 \mathrm{E}-03$ 1. $78002-03$ 8. 4070 -04 $3.9657 \mathrm{E}-04$ 1. $86965-04$ 8. 8153E-05 4. $1567 E-05$ 1. $96035-05$ 9. 2468E-06 4. $36295-06$ 2. 0587E-06 $9.7154 E-07$ 4. $5855 E-07$ 2. $1648 E-07$ 1. 9370 E -07 $1.0000 E+C O$ 1.5977 -02

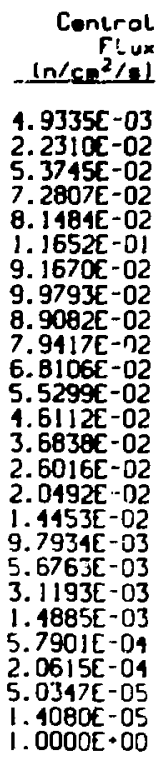

\begin{tabular}{r} 
Control \\
Pdd jount \\
Irhothlel \\
\hline $2.6766 E+00$ \\
$7.6849 E-01$ \\
$7.8423 E-01$ \\
$7.4004 E-01$ \\
$1.3721 E+00$ \\
$1.3533 E+00$ \\
$1.0226 E+00$ \\
$6.7151 E-01$ \\
$6.6586 E-01$ \\
$6.5725 E-01$ \\
$6.4924 E-01$ \\
$9.7173 E-01$ \\
$1.3041 E+00$ \\
$1.3304 E+00$ \\
$1.3631 E+00$ \\
$1.4103 E+00$ \\
$1.4525 E+00$ \\
$1.5745 E+00$ \\
$\vdots .2289 E+00$ \\
$8.9273 E-01$ \\
$8.8710 E-01$ \\
$9.6262 E-01$ \\
$1.0044 E-00$ \\
$6.1490 E-01$ \\
$5.9450 E-01$
\end{tabular}

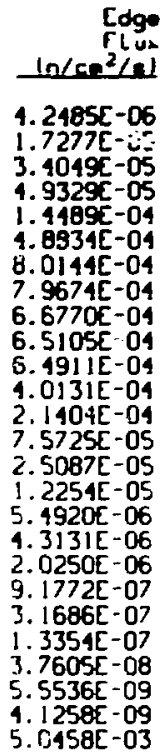

Edge trtogthes

4. $7084[-02$ 8.77965-03 $7.315,4 E-03$ $5.9013 E-03$ 8. $2161 E-03$ E. $4471 E-03$ 4. $2903 E-03$ 1.6973E-03 $1.0998 E-D$ ? 7. $9030 E-04$ 6. 09BOE-DA 6. 30195-D4 7. $18345-04$ $6.75145-04$ $7.0657 k-04$ $7.8536 E-74$ 9.8573E-04 1. 3493E-0. $1.0220[-03$ B. $94365-04$

8. $90535-04$

9. $4142 \mathrm{E}-04$ 8. $1014 E-04$ 6. $3448 E \cdot-14$ 1.20935 - 03

TFaLE B-LXXII]

SAERK-7A 25-GROUP ZONE FISSION FRACTIONS

\begin{tabular}{|c|c|c|c|c|}
\hline op & $\begin{array}{r}\text { Enorgy } \\
\text { Boundory } \\
\text { IevIl }\end{array}$ & $\begin{array}{l}\text { Lothorgy } \\
\text { Hiduh }\end{array}$ & Core & Rerlector \\
\hline $\begin{array}{r}1 \\
2 \\
3 \\
4 \\
5 \\
6 \\
7 \\
8 \\
9 \\
10 \\
11 \\
12 \\
13 \\
14 \\
15 \\
16 \\
17 \\
18 \\
19 \\
20 \\
21\end{array}$ & 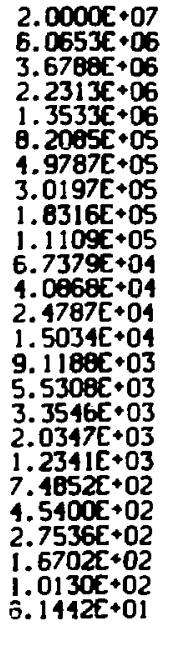 & $\begin{array}{l}1.1931 E+00 \\
5.0000 E-01 \\
5.0000 E-01 \\
5.0000 E-01 \\
5.0000 E-01 \\
5.0000 E-01 \\
5.0000 E-01 \\
5.0000 E-01 \\
5.0000 E-01 \\
5.0000 E-01 \\
5.0000 E-01 \\
5.0000 E-01 \\
5.0000 E-01 \\
5.0000 E-01 \\
5.0000 E-01 \\
5.0000 E-01 \\
5.0000 E-01 \\
5.0000 E-01 \\
5.0000 E-01 \\
5.0000 E-01 \\
5.0000 E-01 \\
5.0000 E-01 \\
5.0000 E-01 \\
5.0000 E-01\end{array}$ & $\begin{array}{l}2.8936 E-02 \\
1.2179 E-01 \\
2.1990 E-01 \\
2.2867 E-01 \\
1.7194 E-01 \\
1.0709 E-01 \\
5.9740 E-02 \\
3.1217 E-02 \\
1.5693 E-02 \\
7.7092 E-03 \\
3.6972 E-03 \\
1.7559 E-03 \\
B .3117 E-04 \\
3.9275 E-04 \\
1.8543 E-04 \\
8.7514 E-05 \\
4.1299 E-05 \\
1.9490 E-05 \\
9.1988 E-06 \\
4.3421 E-06 \\
2.0499 E-06 \\
9.6774 E-07 \\
4.5693 E-07 \\
2.1577 E-07 \\
1.9310 E-07 \\
1.0000 E+00\end{array}$ & $\begin{array}{l}2.4894 E-02 \\
1.1302 E-00 \\
2.1327 E-01 \\
2.2835 E-01 \\
1.7577 E-01 \\
1.1238 E-01 \\
6.4197 E-02 \\
3.4141 E-02 \\
1.7578 E-02 \\
8.6998 E-03 \\
4.1061 E-03 \\
1.9161 E-03 \\
8.9457 E-04 \\
4.1615 E-04 \\
1.9574 E-04 \\
9.1764 E-05 \\
4.3077 E-05 \\
2.0239 E-05 \\
9.5184 E-06 \\
4.4805 E-06 \\
2.1090 E-06 \\
9.9299 E-07 \\
4.6773 E-07 \\
2.2051 E-07 \\
1.9710 E-07 \\
1.0000 E+00\end{array}$ \\
\hline
\end{tabular}


THOLE D-LXXIV

SNCPK-7B 70-EROV IRAWSFORT IHEORY SPECTRA

\begin{tabular}{|c|c|c|c|c|c|c|}
\hline gung & $\begin{array}{r}\text { Enargy } \\
\text { Boundory } \\
\text { InvI }\end{array}$ & $\begin{array}{r}\text { Lethorgy } \\
\text { Hidih }\end{array}$ & $\begin{array}{r}\text { Rooctor } \\
\text { fievion } \\
\text { Froctions }\end{array}$ & $\begin{array}{r}\text { Centrol } \\
\text { Flux } \\
\ln \left\langle a e^{2} /{ }^{2}\right| \\
\end{array}$ & $\begin{array}{r}\text { Control } \\
\text { Adjoint } \\
\text { Irbotolel }\end{array}$ & $\log ^{2}$ \\
\hline $\begin{array}{l}1 \\
2 \\
3 \\
4 \\
5 \\
6 \\
7 \\
8 \\
9 \\
10 \\
11 \\
12 \\
13 \\
11 \\
15 \\
16 \\
17 \\
18 \\
19 \\
20 \\
21 \\
22 \\
23 \\
24 \\
25 \\
26 \\
27 \\
28 \\
29 \\
30 \\
31 \\
32 \\
33 \\
34 \\
35 \\
36 \\
37 \\
30 \\
39 \\
10 \\
41 \\
12 \\
13 \\
14 \\
45 \\
16 \\
47 \\
16 \\
49 \\
50 \\
51 \\
52 \\
53 \\
51 \\
55 \\
56 \\
57 \\
58 \\
59 \\
60 \\
61 \\
62 \\
63 \\
54 \\
65 \\
66 \\
67 \\
68 \\
69 \\
70\end{array}$ & 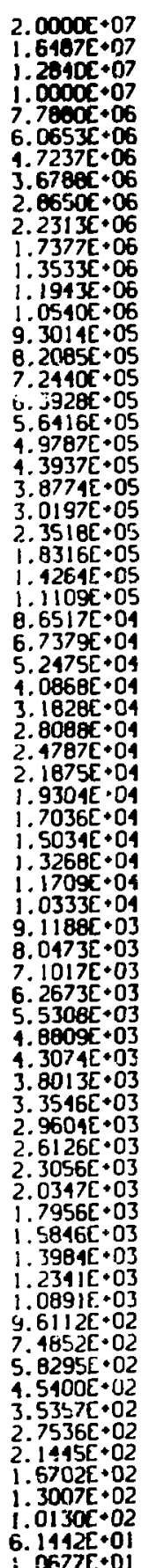 & 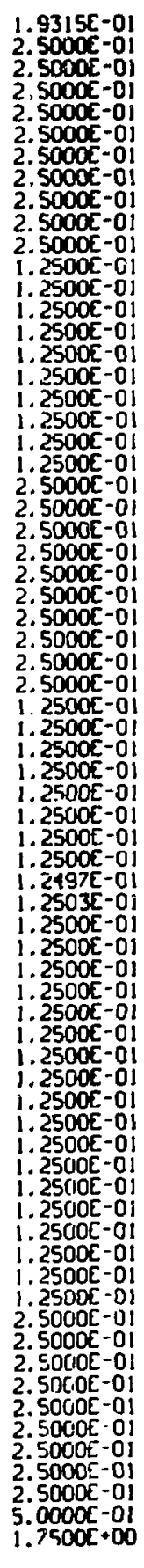 & 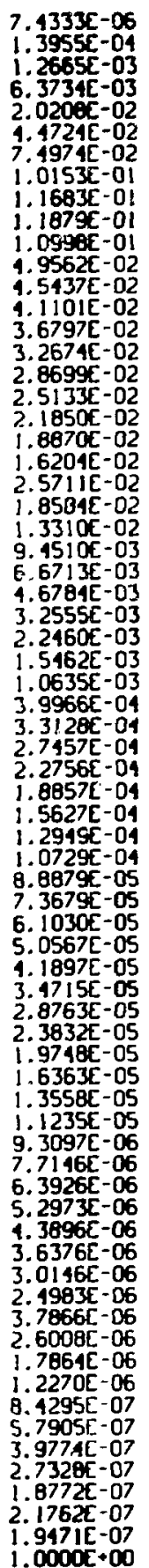 & 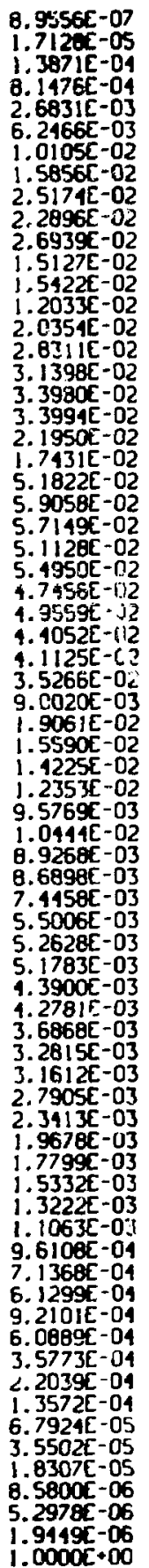 & 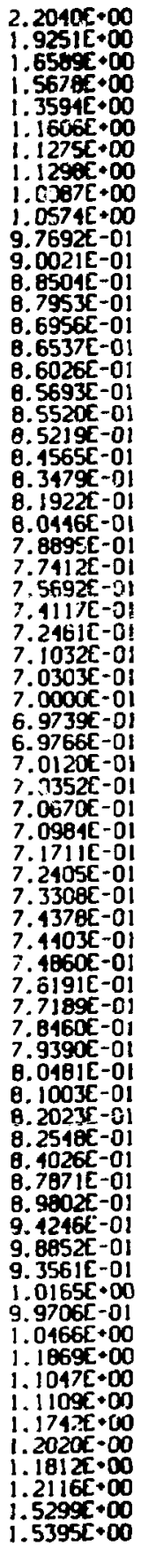 & 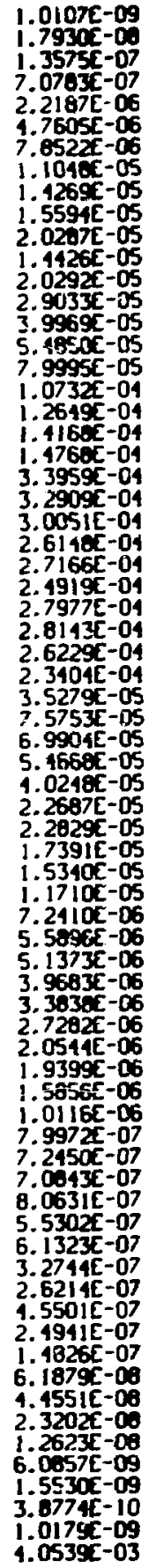 \\
\hline
\end{tabular}

Edip
ind joine
(nholal

$2.9035-02$ 2. $152 x-02$ $1.997 \mathrm{a}-02$ $1.6505 \mathrm{c}-02$ 1. $3543 \mathrm{z}-02$ 1.0255E-02 0. 5.0705-03 0.25302-03 $7.7242 x-03$ 6.9372x-03 5.6579:-03

4. $1910<-03$ 4. $1.055-03$ $4.59765-03$ 4. $6294 \mathrm{E}-03$ 4. $0.59 \mathrm{t}-03$

1. $0912 t-03$ 4. $4351 E-03$

3. $9049 \mathrm{E}-03$

3. 4254[-03 2. $9795 \mathrm{E}-03$

2. $6000,-03$

1. $9072 x-03$

1. $50.0 .5-03$

$1.2160-03$

1. $0312 \alpha-03$

8.6162t-04

$7.52675-04$

$6.73608-04$

5. $7347[-04$

4.7969t-04

$4.06135-04$

4. 3560 [-04

1. 1 boot-04

3.8700:-04

3. $5999 \mathrm{c}-04$

3. 36.0 -04

3. $4933 \mathrm{E}-04$

3. $5000 \mathrm{~L}-04$

3.6265x-04

3. 6000E-04

3. $6474 E-04$

$3.67125-04$

3. $0562 x-04$

3. 900iE-0

4. 1366:-04

4. 23495-04

4. 19.9:-0.

4. $1057 \mathrm{E}-0 \mathrm{~s}$

4. 140.5-0.

1.0925 -0 .

5. $030-0$

6. $2165=04$

7. $113 \%-04$

$7.23235-04$

7. $20055-01$

7. cosot-04

$6.53 \times 05-04$

7. 51045-04

8.1695-04

0.9solf-04

$1.06202-03$

9.537\%-01

1.0146E-03

$1.065 \pi-03$

$1.00255-03$

$1.0505 x-03$

6.04302-04

1.05724-03

2. $6505 x-03$ 
TPBLE B-LXXY

SAEPh-78 70-GROUP ZONE CISSION FRACT!ONS

\begin{tabular}{|c|c|c|c|c|}
\hline conos & $\begin{array}{r}\begin{array}{r}\text { Energy } \\
\text { Boundory } \\
\text { IevI }\end{array} \\
\end{array}$ & $\begin{array}{l}\text { Lechorgy } \\
\text { Hudis }\end{array}$ & Cors & Reltenctor \\
\hline $\begin{array}{l}1 \\
2 \\
3 \\
4 \\
5 \\
5 \\
7 \\
8 \\
9 \\
10 \\
11 \\
12 \\
13 \\
34 \\
15 \\
16 \\
17 \\
18 \\
19 \\
20 \\
21 \\
22 \\
23 \\
24 \\
25 \\
26 \\
27 \\
28 \\
29 \\
30 \\
31 \\
32 \\
33 \\
34 \\
35 \\
36 \\
37 \\
38 \\
30 \\
40 \\
41 \\
42 \\
43 \\
44 \\
45 \\
46 \\
47 \\
48 \\
49 \\
51 \\
51 \\
52 \\
53 \\
54 \\
53 \\
56 \\
57 \\
58 \\
59 \\
60 \\
61 \\
62 \\
63 \\
65 \\
60 \\
67 \\
68 \\
69 \\
70\end{array}$ & 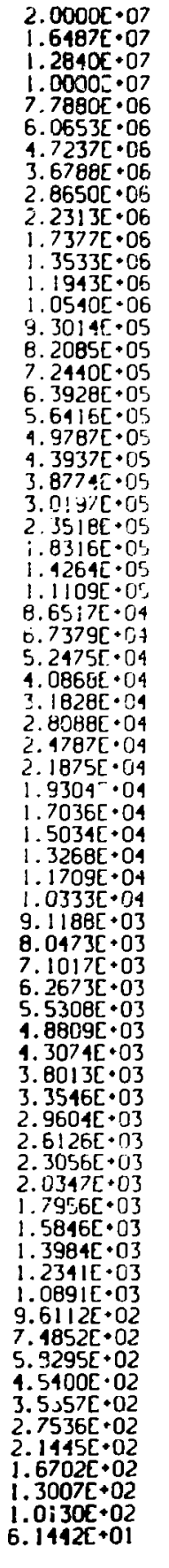 & 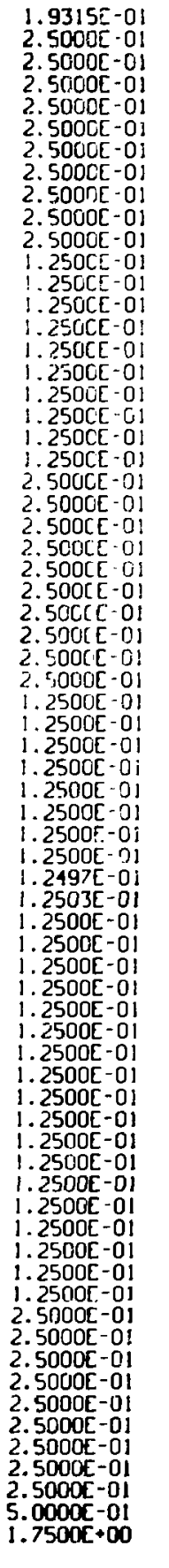 & 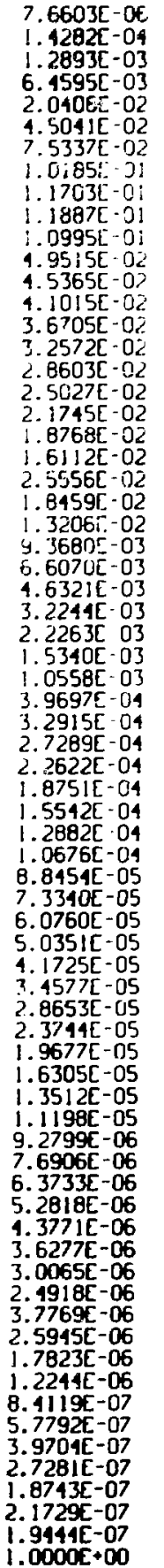 & 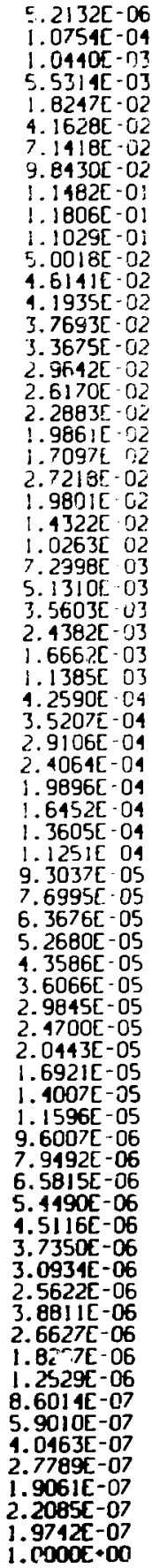 \\
\hline
\end{tabular}


TRBLE B-LXXYI

SKERK-78 25-GROUP TRANSPORT THEOAY SPECTRA

\begin{tabular}{|c|c|c|}
\hline roup & $\begin{array}{r}\text { Energy } \\
\text { Boundory } \\
\text { (eVI) }\end{array}$ & $\begin{array}{r}\text { Lelhor } 9 y \\
\text { Hedis }\end{array}$ \\
\hline $\begin{array}{l}1 \\
2 \\
3 \\
4 \\
5 \\
6 \\
7 \\
8 \\
9 \\
10 \\
11 \\
12 \\
13 \\
14 \\
15 \\
16 \\
17 \\
18 \\
19 \\
20 \\
21 \\
22 \\
23 \\
24 \\
25\end{array}$ & $\begin{array}{l}2.0000 E+07 \\
6.0653 E=06 \\
3.6786 E=06 \\
2.2313 E+06 \\
1.3533 E+06 \\
8.2085 E+05 \\
4.9787 E=05 \\
3.0197 E=05 \\
1.8316 E+05 \\
1.1109 E+05 \\
6.7379 E+04 \\
4.0868 E+04 \\
2.4787 E+04 \\
1.5034 E+04 \\
9.1188 E=03 \\
5.5308 E \cdot 03 \\
3.3546 E+03 \\
2.0347 E \cdot 03 \\
1.2341 E+03 \\
7.4852 E+02 \\
4.5400 E+02 \\
2.7536 E+02 \\
1.6702 E+02 \\
1.0130 E+02 \\
6.1442 E+01 \\
1.0677 E+01\end{array}$ & 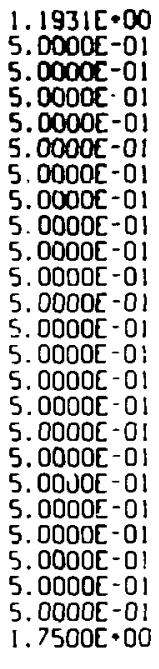 \\
\hline
\end{tabular}

\begin{tabular}{|c|c|c|}
\hline $\begin{array}{r}\text { Reoctor } \\
\text { Fusicon } \\
\text { frocluone }\end{array}$ & $\begin{array}{r}\text { Conirol } \\
7 L u x \\
\text { In/cesel } \\
\end{array}$ & $\begin{array}{r}\text { Centrol } \\
\text { Ad jount } \\
\text { (rholntel }\end{array}$ \\
\hline 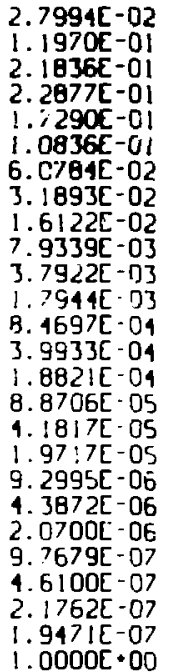 & $\begin{array}{l}3.6546 E-03 \\
1.6352 E-02 \\
4.1030 E-02 \\
4.9835 E-02 \\
6.2936 E-02 \\
1.2768 E-01 \\
9.1204 E-02 \\
1.1621 E-01 \\
1.060 B E-01 \\
9.7015 E-02 \\
8.5177 E-02 \\
5.332 B E-02 \\
5.1745 E-02 \\
3.5506 E-02 \\
2.0332 E-02 \\
1.4408 E-02 \\
9.8795 E-03 \\
4.9227 E-03 \\
2.2477 E-03 \\
9.6663 E-04 \\
3.5612 E-04 \\
1.0343 E-01 \\
2.6687 E-05 \\
5.2978 E-06 \\
1.9449 E-06 \\
1.0000 E+00\end{array}$ & $\begin{array}{l}3.2143 E+00 \\
8.4388 E-01 \\
8.2191 E-01 \\
7.5028 E-01 \\
1.3035 E-00 \\
1.2679 c+00 \\
9.3406 E-01 \\
5.9883 E-01 \\
5.7648 E-01 \\
5.5251 E-01 \\
5.2925[-0 ! \\
7.7466[-01 \\
1.0360[-00 \\
1.0637[-00 \\
1.1058[-00 \\
1.1637[-00 \\
1.2156[+00 \\
1.3674[-00 \\
1.0877[-00 \\
8.2373[-01 \\
8.1714[-01 \\
8.7638[-01 \\
8.8250[-01 \\
5.6425[-0 \\
5.6777[-01\end{array}$ \\
\hline
\end{tabular}

\begin{tabular}{|c|c|}
\hline $\begin{array}{r}\text { Eoge } \\
F \operatorname{lu} 1 \\
\left.\ln / \mathrm{am}^{2} / \mathrm{l}\right)\end{array}$ & $\begin{array}{r}\text { Edge } \\
\text { Rd jount } \\
\text { Irhotheri }\end{array}$ \\
\hline $\begin{array}{l}3.0812 E-05 \\
1.2613 E-05 \\
2.5316 E-05 \\
3.5881 E-05 \\
1.0372 E-04 \\
3.6865 E-04 \\
6.2895 E-04 \\
6.2960 E-04 \\
5.3313 E-04 \\
5.2895 E-E \\
5.4371 E-04 \\
3.4507 E-04 \\
1.9751 E-04 \\
6.7269 E-05 \\
2.1936 E-05 \\
1.6106 E-05 \\
4.1214 E-06 \\
2.6810[-06 \\
1.0446[-06 \\
3.9767 E-07 \\
1.0643 E-07 \\
3.5826[-08 \\
7.6387 E-09 \\
3.8774 E-10 \\
1.0179[-09 \\
4.0539[-03\end{array}$ & $\begin{array}{l}3.8372[-02 \\
7.0967[-0] \\
5.89-8 E-03 \\
4.7226[-03 \\
6.5665[-03 \\
6.7187[-0] \\
3.3244[-0] \\
1.2894[-03 \\
8.2907[-04 \\
5.9544[-04 \\
4.5993[-04 \\
4.7958[-04 \\
5.5391[-04 \\
5.2745[-04 \\
5.5893[-04 \\
6.2611[-04 \\
1.8688[-04 \\
1.0855[-0] \\
8.2312[-04 \\
7.2354[-6.4 \\
7.2597[-04 \\
7.6319[-04 \\
6.8411[-04 \\
6.8863[-04 \\
9.775][-04\end{array}$ \\
\hline
\end{tabular}

TABLE B-LXXY)/

SHEAK-79 25-GROUP ZONE FIS5ION I RACTION5

\begin{tabular}{|c|c|c|c|c|}
\hline ceng: & $\begin{array}{r}\text { Energy } \\
\text { Boundory } \\
\text { Ley! }\end{array}$ & $\begin{array}{r}\text { Lolhorgy } \\
H_{\text {idth }}\end{array}$ & Core & Reflecter \\
\hline $\begin{array}{l}1 \\
2 \\
3 \\
4 \\
5 \\
6 \\
7 \\
8 \\
9 \\
10 \\
11 \\
12 \\
13 \\
14 \\
15 \\
16 \\
17 \\
18 \\
19 \\
20 \\
21 \\
22 \\
23 \\
24 \\
25\end{array}$ & $\begin{array}{l}2.0000 E \cdot 07 \\
6.0653 E+06 \\
3.6788 E+06 \\
2.2313 E+06 \\
1.3533 E+06 \\
8.2085 E+05 \\
1.9787 E+05 \\
3.0197 E+05 \\
1.8316 E+05 \\
1.1109 E+05 \\
6.7379 E+04 \\
1.0868 E+04 \\
2.4787 E+04 \\
1.5034 E+04 \\
9.1188 E+03 \\
5.5308 E+03 \\
3.3546 E+03 \\
2.0347 E+03 \\
1.2341 E+03 \\
7.4852 E+02 \\
1.5400 E+02 \\
2.7536 E+02 \\
1.6702 E+02 \\
1.0130 E+02 \\
6.1442 E+01\end{array}$ & $\begin{array}{l}1.1931[+00 \\
5.0000 E-01 \\
5.0000 E-01 \\
5.0000 E-01 \\
5.0000 E-01 \\
5.0000 E-01 \\
5.0000 E-01 \\
5.0000 E-01 \\
5.0000 E-01 \\
5.0000 E-01 \\
5.0000 E-01 \\
5.0000 E-01 \\
5.0000 E-01 \\
5.0000 E-01 \\
5.0000 E-01 \\
5.0000 E-01 \\
5.0000 E-01 \\
5.0000 E-01 \\
5.0000 E-01 \\
5.0000 E-01 \\
5.0000 E-01 \\
5.0000 E-01 \\
5.0000 E-01 \\
5.0000 E-01 \\
1.7500 E+00\end{array}$ & $\begin{array}{l}2.8307 E-02 \\
1.2038 E-01 \\
2.1688 E-01 \\
2.28 B 2 E-01 \\
1.7260 E-01 \\
1.0795 E-01 \\
6.0437 E-02 \\
3.1665 E-02 \\
1.5975 E-02 \\
7.0565 E-03 \\
3.7603 E-03 \\
1.7819 E-03 \\
8.4204 E-04 \\
3.9736 E-04 \\
1.9741 E-04 \\
8.8379 E-05 \\
1.1681 E-05 \\
1.9660 E-05 \\
9.2753 E-06 \\
4.3768 E-06 \\
2.0656 E-06 \\
9.7496 E-07 \\
4.6024 E-07 \\
2.1729 E-07 \\
1.9444 E-07 \\
1.0000 E-00\end{array}$ & $\begin{array}{l}2.4935 E-02 \\
1.1305 E-01 \\
2.1325 E-01 \\
2.2835 E-01 \\
1.7579 E-01 \\
1.1237 E-01 \\
6.4176 E-02 \\
3.4123 E-02 \\
1.7563 E-02 \\
8.6913 E-03 \\
4.1041 E-03 \\
1.9165 E-03 \\
8.9517 E-04 \\
1.1859 E-04 \\
1.9601 E-04 \\
9.1910 E-05 \\
4.3153 E-05 \\
2.0277 E-05 \\
9.5367 E-06 \\
4.4894 E-06 \\
2.113 C E-06 \\
9.9471 E-07 \\
4.6649 E-07 \\
2.2085 E-07 \\
1.9742 E-07 \\
1.0000 E-00\end{array}$ \\
\hline
\end{tabular}


Tface $8-1 . x \times y \mid 1]$

ZPh-g-31 70-GKOWP TRFASPCRT IHETRI SPECTRA

\begin{tabular}{|c|c|c|c|c|c|c|c|}
\hline a & $\begin{array}{r}\text { Energy } \\
\text { Boundory } \\
\text { (el) }\end{array}$ & $\begin{array}{r}\text { Lothorgy } \\
\text { Hedilh }\end{array}$ & $\begin{array}{r}\text { Rooctor } \\
\text { rueven } \\
\text { Erocheon }\end{array}$ & $\begin{array}{r}\text { Control } \\
F l \text { un } \\
\ln \tan ^{2} / \mathrm{L}\end{array}$ & $\begin{array}{r}\text { Control } \\
\text { Ad pont } \\
\text { ectogtolel } \\
\end{array}$ & $\begin{array}{r}\text { Cogo } \\
\text { Flux } \\
\ln \left(\cos ^{2} / \operatorname{lat}\right.\end{array}$ & $\begin{array}{l}\text { Coge } \\
\text { Ad pint } \\
\text { ichololes }\end{array}$ \\
\hline $\begin{array}{l}1 \\
2 \\
3 \\
1 \\
5 \\
6 \\
7 \\
8 \\
9 \\
10 \\
11 \\
12 \\
13 \\
14 \\
15 \\
16 \\
17 \\
18 \\
19 \\
20 \\
21 \\
22 \\
23 \\
24 \\
25 \\
26 \\
27 \\
28 \\
29 \\
30 \\
31 \\
32 \\
33 \\
34 \\
35 \\
36 \\
37 \\
38 \\
39 \\
10 \\
41 \\
42 \\
13 \\
11 \\
15 \\
16 \\
17 \\
18 \\
49 \\
50 \\
51 \\
52 \\
53 \\
54 \\
55 \\
55 \\
57 \\
58 \\
59 \\
60 \\
61 \\
62 \\
63 \\
64 \\
65 \\
66 \\
67 \\
68 \\
69 \\
70\end{array}$ & 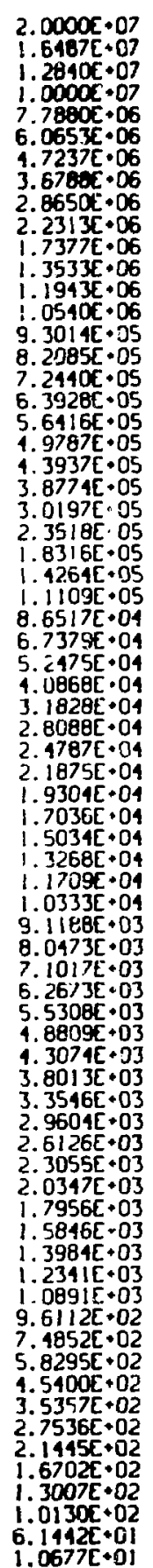 & 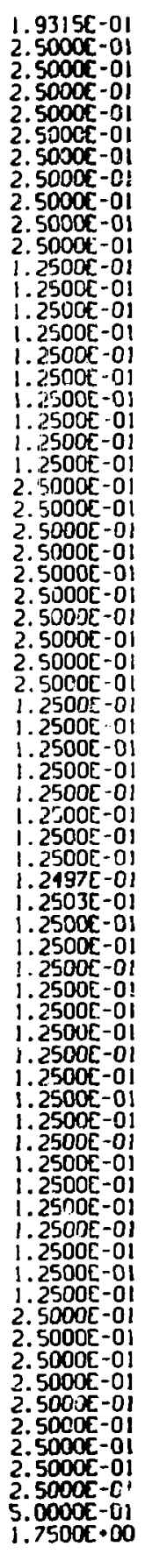 & 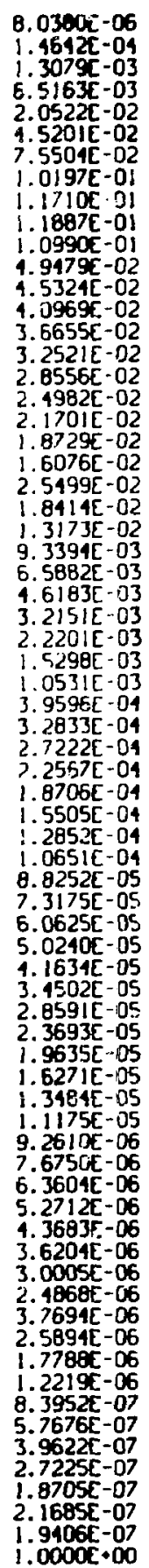 & 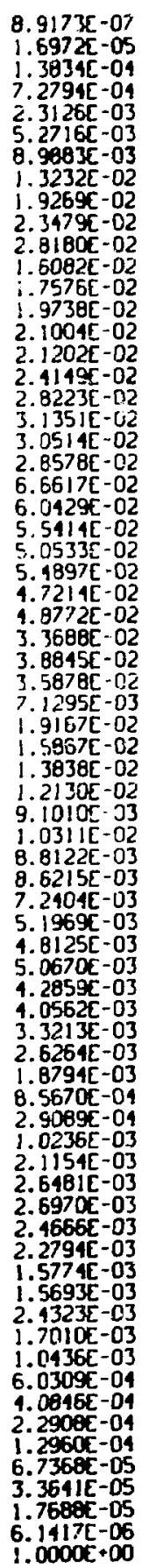 & 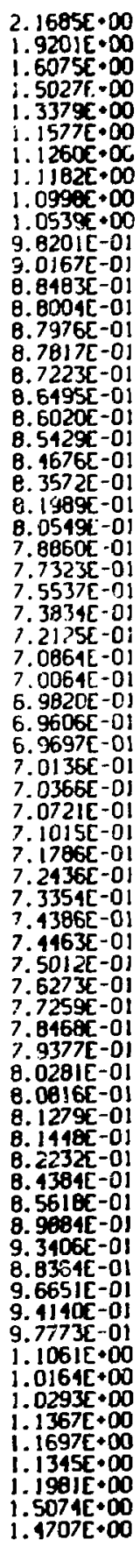 & 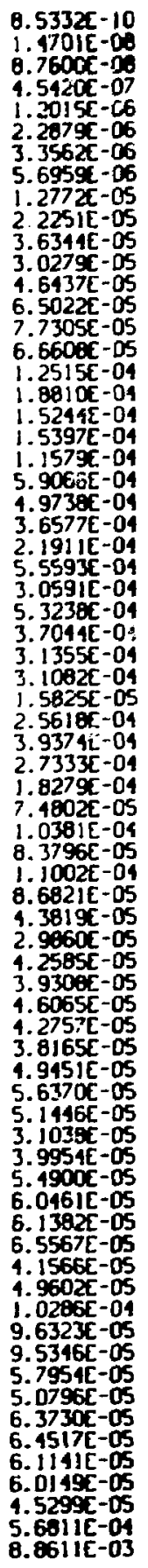 & 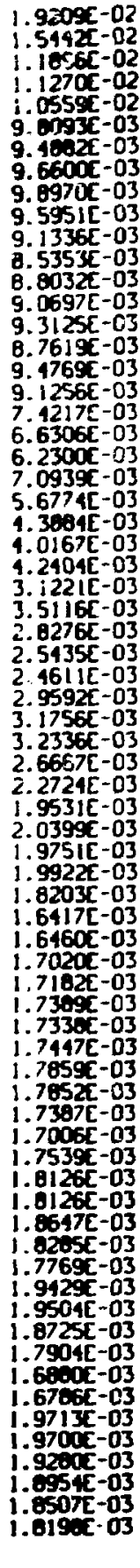 \\
\hline
\end{tabular}


Thed $B-L x x \mid X$

2PR-9-3! 70-GRCOP ZONC FISSION TRACTIONS

\begin{tabular}{|c|c|c|c|c|c|}
\hline ceng & $\begin{array}{r}\text { Energy } \\
\text { Boundory } \\
\ln y\end{array}$ & $\begin{array}{r}\text { Lethorgy } \\
\text { Hudth }\end{array}$ & Core & Blonkes & Reflector \\
\hline $\begin{array}{l}1 \\
2 \\
3 \\
4 \\
5 \\
6 \\
7 \\
8 \\
9 \\
10 \\
11 \\
12 \\
13 \\
14 \\
15 \\
16 \\
17 \\
18 \\
19 \\
20 \\
21 \\
22 \\
21 \\
54 \\
25 \\
26 \\
23 \\
28 \\
29 \\
30 \\
31 \\
32 \\
33 \\
13 \\
15 \\
36 \\
19 \\
38 \\
39 \\
40 \\
41 \\
42 \\
43 \\
44 \\
45 \\
46 \\
47 \\
48 \\
49 \\
50 \\
51 \\
52 \\
53 \\
54 \\
55 \\
56 \\
57 \\
58 \\
59 \\
60 \\
61 \\
62 \\
63 \\
64 \\
65 \\
66 \\
67 \\
68 \\
69 \\
70\end{array}$ & 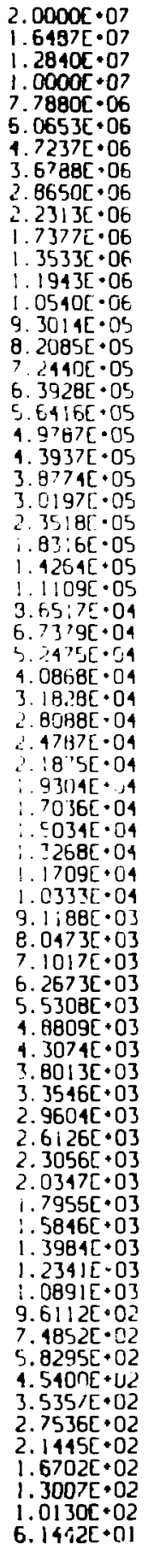 & 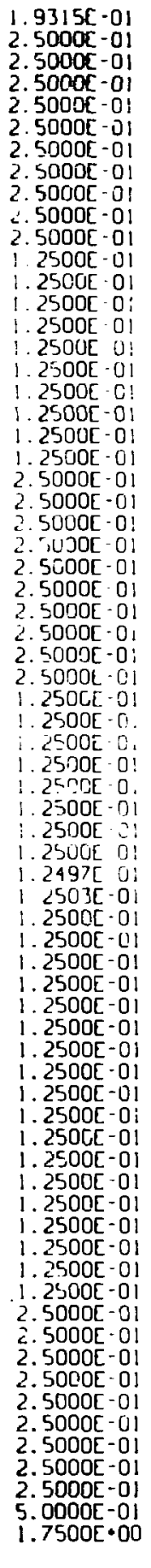 & 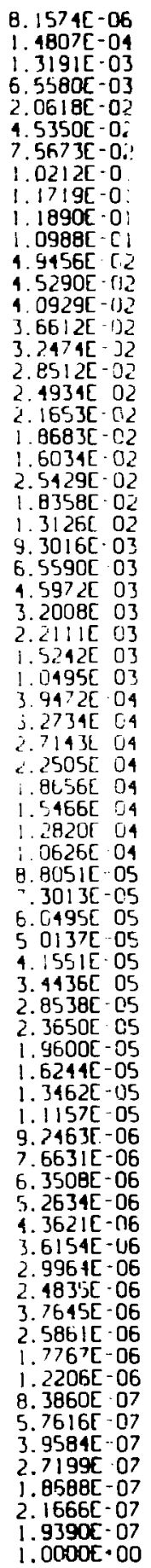 & 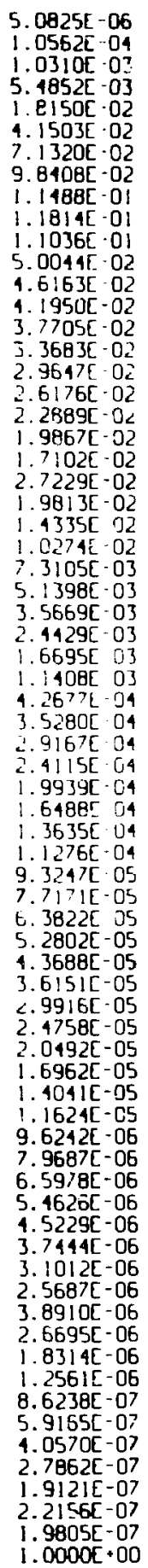 & 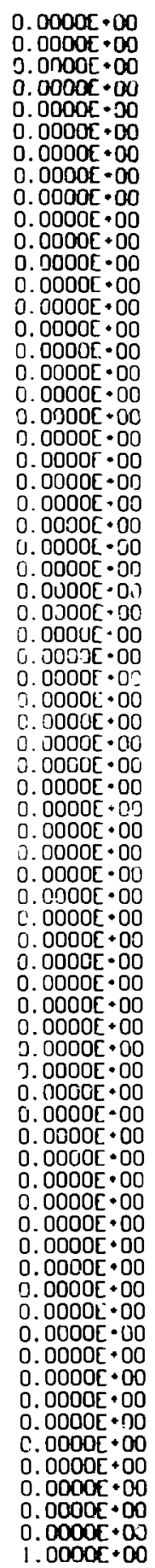 \\
\hline
\end{tabular}


IABLE $B-i \times X X$

2PR-9-31 25-GROUP TRANSPORT IHEORY SPECTRA

\begin{tabular}{|c|c|}
\hline Gersun & $\begin{array}{r}\text { Energy } \\
\text { Boundory } \\
\text { Iey } \\
\end{array}$ \\
\hline $\begin{array}{l}1 \\
2 \\
3 \\
4 \\
5 \\
6 \\
7 \\
8 \\
9 \\
10 \\
11 \\
12 \\
13 \\
14 \\
15 \\
16 \\
17 \\
19 \\
19 \\
20 \\
21 \\
22 \\
23 \\
24 \\
25\end{array}$ & $\begin{array}{l}2.0000 E+07 \\
6.0653 E+06 \\
3.678 E E+06 \\
2.2313 E+06 \\
1.3533 E+06 \\
8.2035 E+05 \\
1.9787 E+05 \\
3.0197 E+05 \\
1.8316 E+05 \\
1.1109 E+05 \\
6.7379 E+01 \\
1.08 E \theta E+04 \\
2.4787 E+04 \\
1.5034 E+04 \\
9.1188 E+03 \\
5.5308 E+03 \\
3.3546 E+03 \\
2.0347 E+03 \\
1.2341 E+03 \\
7.4952 E+02 \\
1.5400 E+02 \\
2.7536 E+02 \\
1.6702 E+02 \\
1.0130 E+02 \\
6.1442 E+01 \\
1.0677 E+01\end{array}$ \\
\hline
\end{tabular}

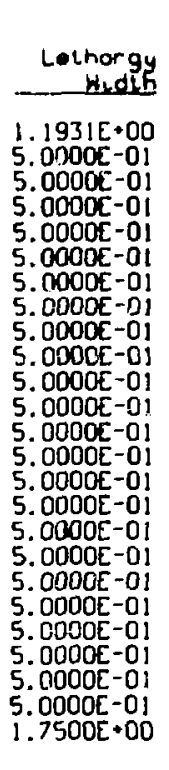

Conlrot
rLuE
In/sm 2 (n)
$3.1968 E-03$
$1.4260 E-02$
$3.2520 E-02$
$5.1660 E-02$
$7.1400 E-02$
$1.0493 E-01$
$1.2571 E-01$
$1.1584 E-01$
$1.0543 E-01$
$9.5986 E-02$
$7.2534 E-02$
$6.2175 E-02$
$5.0936 E-02$
$3.4985 E-02$
$1.9362 E-02$
$1.1883 E-02$
$4.2066 E-03$
$1.0991 E-02$
$5.5790 E-03$
$2.7446 E-03$
$1.0116 E-03$
$3.5868 E-04$
$1.0101 E-04$
$1.7686 E-05$
$6.1417 E-06$
$1.0000 E+00$

\begin{tabular}{r} 
Centrol \\
Ad jolnt \\
irholn $\angle 1 \mid$ \\
\hline $3.1480 E+00$ \\
$6.4217 E-01$ \\
$6.1793 E-01$ \\
$7.5077 E-01$ \\
$1.3078 E+00$ \\
$1.2817 E+00$ \\
$9.3548 E-01$ \\
$5.9939 E-01$ \\
$5.7595 E-01$ \\
$5.5083 E-01$ \\
$5.2730 E-01$ \\
$7.7254 E-01$ \\
$1.0359 E+00$ \\
$1.0642 E+00$ \\
$1.1068 E+00$ \\
$1.1630 E+00$ \\
$1.2014 E+00$ \\
$1.3026 E+00$ \\
$1.0294 E+00$ \\
$7.6846 E-01$ \\
$7.5437 E-01$ \\
$8.5054 E-01$ \\
$8.6019 E-01$ \\
$5.5587 E-01$ \\
$5.4235 E-01$ \\
\end{tabular}

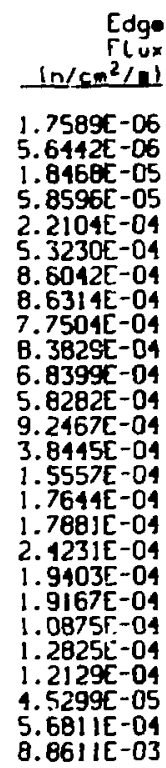

Edgo

Rod oLn trhoth/s!

2. $5215[-02$

7. $11635-03$

$7.21=\mathrm{CT}-03$

6.9065E-03

1. $31735-02$

1. $2828 \mathrm{E}-02$

$7.3566 \mathrm{E}-03$

$3.7119[-03$

3.0450E -03

2. $4463 \mathrm{E}-03$

1. $9807 E-03$

3. $16995-03$

3. $7341 E-03$

2. 8665E-03

$2.4737 \mathrm{E}-03$

2. $5826 \mathrm{E}-03$

$2.5734 \mathrm{E}-03$

2.6988E-03

2. 0910ट-03

1. $3507 \mathrm{E}-03$

i. $2415 E-03$

1. $4534 E 03$

1. $4099 \mathrm{E}-03$

$6.62495-34$

$6.7108 E-04$

TABLE $B-L \times \times X 1$

ZPR-9-31 25-GROUP ZONE FISSION FRACTIOAS

\begin{tabular}{|c|c|c|c|c|c|}
\hline Q40 & $\begin{array}{r}\text { Energy } \\
\text { Boundory } \\
\text { IEYI) }\end{array}$ & $\begin{array}{r}\text { Lethorgy } \\
\text { Hidith }\end{array}$ & Core & Blontant & Reflecier \\
\hline $\begin{array}{r}1 \\
2 \\
3 \\
4 \\
5 \\
6 \\
7 \\
9 \\
9 \\
9 \\
10 \\
11 \\
12 \\
13 \\
14 \\
15 \\
16 \\
17 \\
18 \\
19 \\
20 \\
21 \\
22 \\
23 \\
24 \\
25\end{array}$ & $\begin{array}{l}2.0000 E+07 \\
6.0653 E+06 \\
3.6786 E+05 \\
2.2313 E+06 \\
1.3533 E+06 \\
8.2005 E+05 \\
4.9787 E+05 \\
3.0197 E+05 \\
1.8316 E+05 \\
1.1109 E+05 \\
6.7379 E+04 \\
4.0868 E+04 \\
2.4787 E+04 \\
1.5034 E+04 \\
9.1168 E+03 \\
5.5306 E+03 \\
3.3546 E+03 \\
2.0347 E+03 \\
1.2341 E+03 \\
7.4852 E+02 \\
4.5400 E+02 \\
2.7536 E+02 \\
1.6702 E+02 \\
1.0130 E+02 \\
6.1442 E+01\end{array}$ & 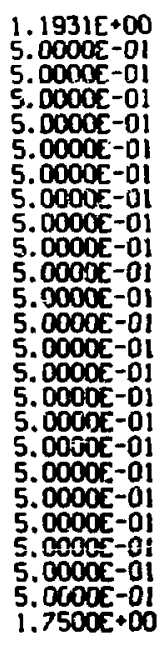 & 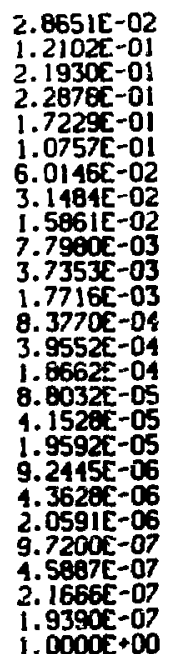 & 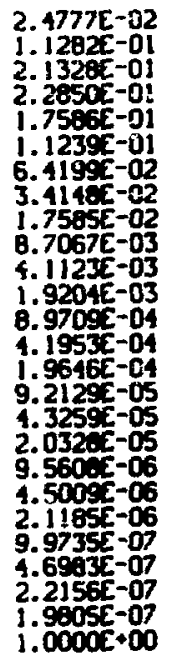 & 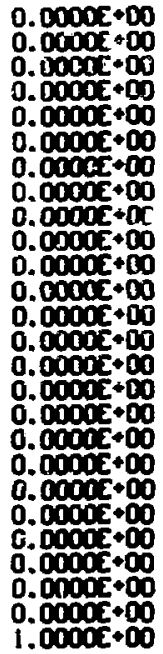 \\
\hline
\end{tabular}


TFBu $8-L \times \times \times 11$

JEZEBEL. CENTRRL AVERARE CROSS SECTIONS

\begin{tabular}{|c|c|c|c|c|}
\hline 1 & Finfion & Conture & Mv & noorl \\
\hline 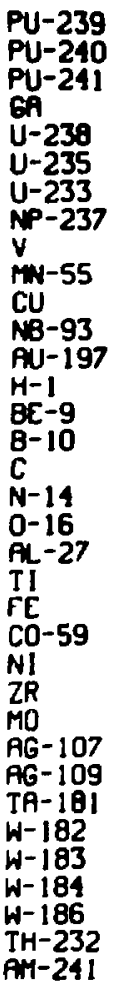 & 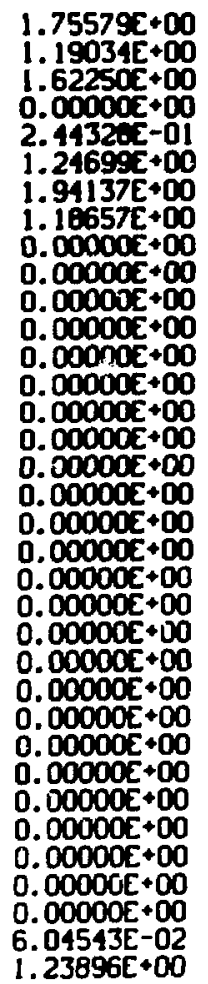 & 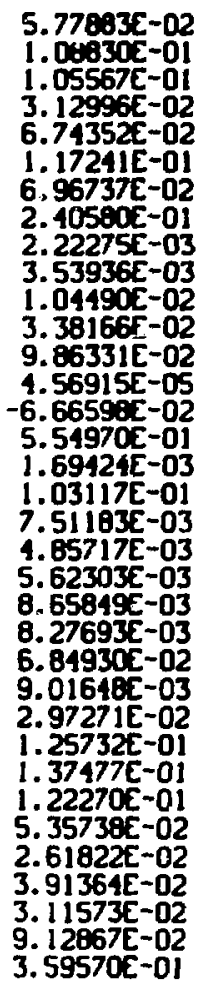 & 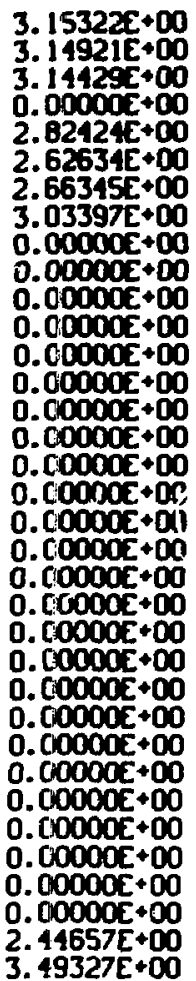 & $\begin{array}{l}5.3831 \\
5.4581 \\
3.6612 \\
5.3716 \\
5.2471 \\
4.9997 \\
5.6081 \\
3.0058 \\
2.8674 \\
2.6532 \\
4.2148 \\
4.6268 \\
1.3760 \\
2.3014 \\
2.3930 \\
2.2154 \\
1.8661 \\
2.6650 \\
2.3573 \\
2.4082 \\
2.5007 \\
2.0307 \\
2.8972 \\
4.3226 \\
4\end{array}$ \\
\hline
\end{tabular}

TFBLE B-LXXX1I]

VERA-IIA CENTRPL AVERFEE CHOSS SECTIONS

\begin{tabular}{|c|c|c|c|c|}
\hline 014,210 & Firnion & Coplure & Nu & port \\
\hline $\begin{array}{l}P U-239 \\
P U-240 \\
P U-241 \\
6 A \\
C \\
F E \\
C R \\
N I \\
C U \\
U-235 \\
U-238 \\
P B \\
S N \\
N P-237 \\
U-233\end{array}$ & $\begin{array}{l}1.71926 E+00 \\
7.86634 E-01 \\
1.91695 E+00 \\
0.00000 E+00 \\
0.00000 E+00 \\
0.00000 E+00 \\
0.00000 E+00 \\
0.00000 E+00 \\
0.00000 E+00 \\
1.15611 E+00 \\
1.33609 E-01 \\
0.00000 E+00 \\
0.00000 E+00 \\
7.68953 E-01 \\
2.21245 E+00\end{array}$ & 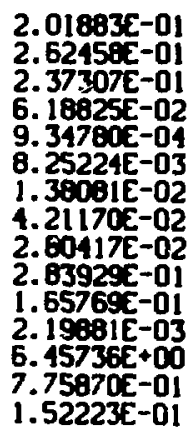 & 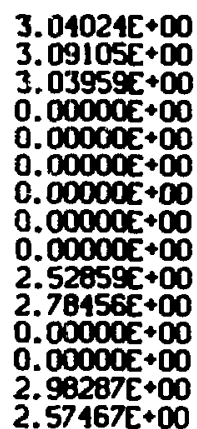 & $\begin{array}{l}6 \\
1 \\
2 \\
2 \\
3 \\
3\end{array}$ \\
\hline
\end{tabular}


TABLE B-LXXXIV

2PR-3-48 CENTRAL AVERAGE CROSS SECTIONS

\begin{tabular}{|c|c|c|c|c|}
\hline rial & Fincion & Copture & Nu & Ironsport \\
\hline $\begin{array}{l}-239 \\
j-240 \\
j-241 \\
-242 \\
-235 \\
238 \\
1-23 \\
\\
\\
-27 \\
1-55 \\
10 \\
10 \\
-181\end{array}$ & $\begin{array}{l}1.80718 E+00 \\
4.91651 E-01 \\
2.40272 E+00 \\
3.68703 E+01 \\
1.82655 E+00 \\
6.38961 E-02 \\
0.00000 E+00 \\
0.00000 E+00 \\
0.00000 E+00 \\
0.00000 E+00 \\
0.00000 E+00 \\
0.00000 E+00 \\
0.00000 E+00 \\
0.00000 E+00 \\
0.00000 E+00 \\
0.00000 E+00 \\
0.00000 E+00\end{array}$ & $\begin{array}{l}4.41754 E-01 \\
1.98879 E-01 \\
1.19378 E-01 \\
4.00203 E-01 \\
5.10485 E-01 \\
2.46220 E-01 \\
6.24690 E-01 \\
1.84105 E-03 \\
9.59616 E-03 \\
1.83101 E-02 \\
3.25974 E-02 \\
1.26396 E-01 \\
3.09935 E-03 \\
5.67397 E-02 \\
3.92852 E-03 \\
2.41718 E+00 \\
7.89477 E-01\end{array}$ & $\begin{array}{l}2.96392 E+00 \\
3.03829 E+00 \\
2.98706 E+00 \\
3.06501 E+00 \\
2.47719 E+00 \\
2.76930 E+00 \\
0.00000 E+00 \\
0.00000 E+00 \\
0.00000 E+00 \\
0.00000 E+00 \\
0.00000 E+00 \\
0.00000 E+00 \\
0.00000 E+00 \\
0.00000 E+00 \\
0.00000 E+00 \\
0.00000 E+00 \\
0.00000 E+00\end{array}$ & 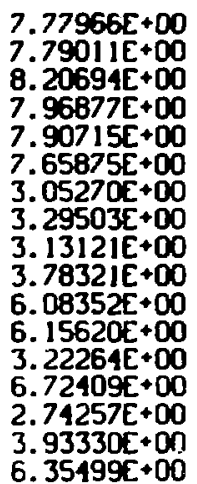 \\
\hline
\end{tabular}

TABLE B-LXXXV

ZEBRA-3 CENTRAL AVERAGE CROSS SECTIONS

\begin{tabular}{|c|c|c|c|c|}
\hline 然 & Fireion & Co & $\mathrm{Nu}$ & port \\
\hline $\begin{array}{l}J-239 \\
j-240 \\
j-241 \\
-235 \\
-238 \\
0 \\
\\
-55 \\
-27 \\
\\
-233 \\
231 \\
236 \\
-237 \\
10 \\
11 \\
-181 \\
-6 \\
-197 \\
-23\end{array}$ & $\begin{array}{l}1.63351 E+00 \\
5.52264 E-01 \\
1.80423 E+00 \\
1.36022 E+00 \\
6.45435 E-02 \\
0.00000 E+00 \\
0.00000 E+00 \\
0.00000 E+00 \\
0.00000 E+00 \\
0.00000 E+00 \\
0.00000 E+00 \\
0.00000 E+00 \\
0.00000 E+00 \\
0.00000 E+00 \\
0.00000 E+00 \\
0.00000 E+00 \\
2.11973 E+00 \\
5.28053 E-01 \\
1.5455 \xi E-01 \\
5.36589 \varepsilon-01 \\
0.00000 E+00 \\
0.00000 E+00 \\
0.00000 E+00 \\
0.00000 E+00 \\
0.00000 E+00 \\
0.00000 E+00 \\
0.00000 E+00\end{array}$ & $\begin{array}{l}1.69067 E-01 \\
2.31504 \mathrm{E}-01 \\
2.13927 \mathrm{E}-01 \\
2.65201 \mathrm{E}-01 \\
1.42383 \mathrm{E}-01 \\
2.16539 \mathrm{E}-02 \\
7.69080 \mathrm{E}-03 \\
8.84478 \mathrm{E}-04 \\
1.07012 \mathrm{E}-02 \\
5.73911 \mathrm{E}-02 \\
7.83096 \mathrm{E}-03 \\
2.64248 \mathrm{E}-02 \\
2.28799 \mathrm{E}-03 \\
7.04830 \mathrm{E}-03 \\
4.24768 \mathrm{E}-03 \\
1.82935 \mathrm{E}-03 \\
1.58079 \mathrm{E}-01 \\
3.10756 \mathrm{E}-01 \\
3.09210 \mathrm{E}-01 \\
6.62612 \mathrm{E}-01 \\
1.14729 \mathrm{E}+00 \\
1.58217 \mathrm{E}-04 \\
2.56079 \mathrm{E}-01 \\
8.08623 \mathrm{E}-01 \\
2.05339 \mathrm{E}-01 \\
1.05255 \mathrm{E}-03 \\
2.62052 \mathrm{E}-03 \\
7.47691 \mathrm{E}-\mathrm{L}\end{array}$ & 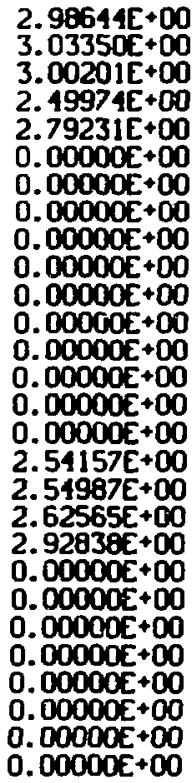 & 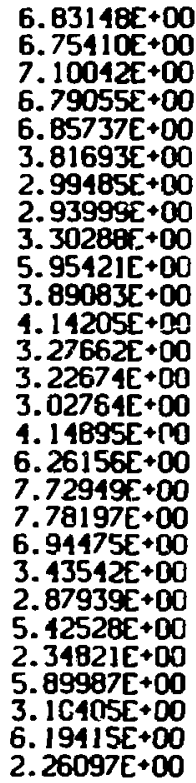 \\
\hline
\end{tabular}


IfBLE B-LXXXYI

GOOIVA CENTRFL AVERAEE CROSS SECTIONS

\begin{tabular}{|c|c|c|c|c|}
\hline theriol & finsion & Conture & Nu & Tronsport \\
\hline $\begin{array}{l}U-235 \\
U-239 \\
U-234 \\
U-233 \\
P U-239 \\
N P-237 \\
T N-55 \\
C O-59 \\
C U \\
A U-197 \\
N B-93 \\
H-1 \\
B E-9 \\
B-10 \\
C \\
A L-27 \\
F E \\
N I \\
T H-232 \\
P U-240\end{array}$ & $\begin{array}{l}1.24818 E+00 \\
2.13002 E+01 \\
1.02730 E+00 \\
1.95613 E+00 \\
1.73995 E+00 \\
1.11165 E+00 \\
0.00000 E+00 \\
0.00000 E+00 \\
0.00000 E+00 \\
0.00000 E+00 \\
0.00000 E+00 \\
0.00000 E+00 \\
0.00000 E+00 \\
0.00000 E+00 \\
0.00000 E+00 \\
0.00000 E+00 \\
0.00000 E+00 \\
0.00000 E+00 \\
5.21682 E-02 \\
1.11530 E+00\end{array}$ & $\begin{array}{r}1.29960 E-01 \\
7.69486 E-02 \\
2.15468 E-01 \\
7.99282 E-02 \\
6.63661 E-02 \\
2.67483 E-01 \\
3.75148 E-03 \\
7.32704 E-03 \\
1.09715 E-02 \\
1.06729 E-01 \\
3.69218 E-02 \\
4.64817 E-05 \\
-3.16129 E-02 \\
5.85682 E-01 \\
1.10358 E-03 \\
3.84452 E-03 \\
7.89684 E-03 \\
5.70077 \mathrm{E}-02 \\
1.02470 E-01 \\
1.17906 \mathrm{E}-01\end{array}$ & 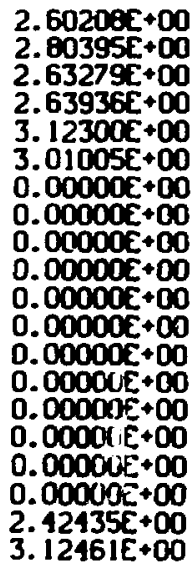 & $\begin{array}{l}5.35282 E+00 \\
5.47722 E+00 \\
6.33196 E+00 \\
5.02313 E+00 \\
5.54783 E+00 \\
5.76166 E+00 \\
2.90205 E+00 \\
2.86690 E+00 \\
2.90733 E+00 \\
1.69411 E+00 \\
4.36046 E+00 \\
1.44862 E+00 \\
2.39517 E+00 \\
2.49574 E+00 \\
2.28417 E+00 \\
2.43458 E+00 \\
2.53210 E+00 \\
2.95382 E+00 \\
5.54526 E+00 \\
5.48241 E+00\end{array}$ \\
\hline
\end{tabular}

TABLE B-LXXXYII

VERA-IG CENTRAL AVERFGE CROSS SECTIONS

\begin{tabular}{|c|c|c|c|c|}
\hline aterio & Finion & Cootuce & $\mathrm{Nu}$ & Ironuport \\
\hline $\begin{array}{l}-235 \\
-236 \\
-234 \\
-238 \\
-1 \\
5 \\
i \\
-233 \\
j-239 \\
j-240 \\
-237 \\
-10 \\
j-197 \\
-27 \\
-23\end{array}$ & $\begin{array}{l}1.49833 E+00 \\
2.68976 E-01 \\
6.84500 E-01 \\
1.22653 E-01 \\
0.00000 E+00 \\
3.00000 E+00 \\
0.00000 E+00 \\
0.00000 E+00 \\
0.00000 E+00 \\
2.26245 E+00 \\
1.73351 E+00 \\
7.51556 E-01 \\
7.31526 E-01 \\
0.00000 E+00 \\
0.00000 E+00 \\
0.00000 E+00 \\
0.00000 E+00\end{array}$ & $\begin{array}{l}3.10784 E-01 \\
3 .+9788 E-01 \\
3.63959 E-01 \\
1.85241 E-01 \\
8.05152 E-04 \\
1.05510 E-04 \\
8.20346 E-03 \\
1.43318 E-02 \\
3.88959 E-02 \\
1.63897 E-01 \\
2.37535 E-01 \\
2.90811 E-01 \\
8.63559 E-01 \\
1.41151 E+00 \\
2.88650 E-01 \\
3.03371 E-03 \\
1.97305 E-03\end{array}$ & $\begin{array}{l}2.51806 \varepsilon+00 \\
2.62807 \varepsilon+00 \\
2.59478 \varepsilon+00 \\
2.76776 \varepsilon+00 \\
0.00000 \varepsilon+00 \\
0.00000 \varepsilon+00 \\
0.00000 \varepsilon+00 \\
0.00000 \varepsilon+00 \\
0.00000 \varepsilon+00 \\
2.56519 \varepsilon+00 \\
3.02587 \varepsilon+00 \\
3.07796 \varepsilon+00 \\
2.97091 \varepsilon+00 \\
0.00000 \varepsilon+00 \\
0.00000 E+00 \\
0.0000 c E+00 \\
0.00000 \varepsilon+00\end{array}$ & $\begin{array}{l}7 . \\
6 . \\
2 . \\
1 \\
2 . \\
3 \\
4 \\
5 . \\
6 . \\
6 .\end{array}$ \\
\hline
\end{tabular}


TFBLE B-LXXXY1II

ZPR-3-6F CENTRPL AVERAGE CROSS SECTIONS

\begin{tabular}{|c|c|c|c|c|}
\hline laterial & Fireion & ure & $N_{4}$ & ore \\
\hline 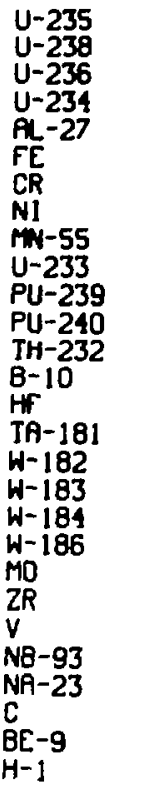 & $\begin{array}{l}1.31783 E+00 \\
1.06307 z-01 \\
2.42602 E-01 \\
6.96796 E-01 \\
0.00000 E+00 \\
0.00000 E+00 \\
0.00000 E+00 \\
0.00 \times 0 E+00 \\
0.00000 E+00 \\
2.06240 E+00 \\
1.66947 E+00 \\
7.43094 E-01 \\
2.55576 E-02 \\
0.00000 E+00 \\
0.00000 E+00 \\
0.00000 E+00 \\
0.00000 E+00 \\
0.00000 E+00 \\
0.00000 E+00 \\
0.00000 E+00 \\
0.00000 E+00 \\
0.00000 E+00 \\
0.00000 E+00 \\
0.00000 E+00 \\
0.00000 E+00 \\
0.00000 E+00 \\
0.00000 E+00 \\
0.00000 E+00\end{array}$ & $\begin{array}{l}2.18659 E-01 \\
1.26883 E-01 \\
2.79384 E-01 \\
2.81203 E-01 \\
2.21392 E-03 \\
7.08471 E-03 \\
1.07060 E-02 \\
3.21112 E-02 \\
6.57262 E-03 \\
1.31920 E-01 \\
1.33393 E-01 \\
1.91512 E-01 \\
1.53073 E-01 \\
9.37860 E-01 \\
2.22216 E-01 \\
2.14431 E-01 \\
8.67683 E-02 \\
9.54036 E-02 \\
7.10429 E-02 \\
6.04691 E-02 \\
4.92009 E-02 \\
1.24151 E-02 \\
3.95017 E-03 \\
6.16111 E-02 \\
1.10498 E-03 \\
9.56222 E-01\end{array}$ & 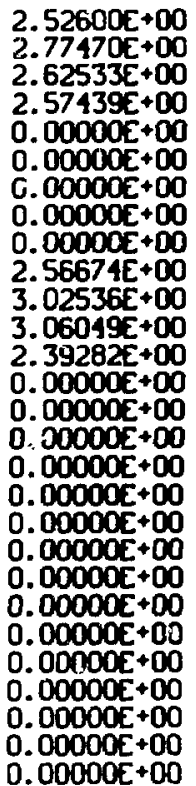 & 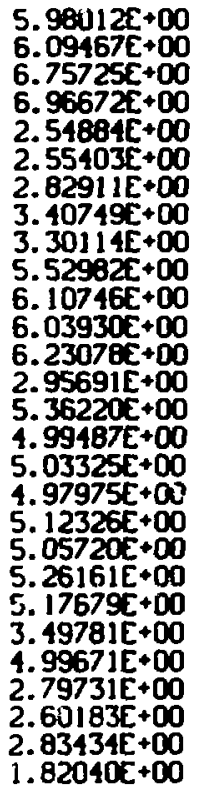 \\
\hline
\end{tabular}

TABLE $B-L \times X X I X$

ZPR-3-11 CENTRAL AVERAGE CROSS SECTIONS

\begin{tabular}{|c|c|c|c|c|}
\hline ateriol & Fiseion & Copture & $\mathrm{Mu}_{4}$ & Ironsport \\
\hline $\begin{array}{l}U-235 \\
U-236 \\
U-238 \\
U-234 \\
F E \\
C R \\
N I \\
N-55 \\
J-233 \\
U-239 \\
U-240 \\
P-237 \\
3-10 \\
P A-181 \\
10 \\
A-23 \\
H-27 \\
U-16\end{array}$ & $\begin{array}{l}1.36537 E+00 \\
1.36884 E-01 \\
5.57476 E-02 \\
4.93518 E-01 \\
0.00000 E+00 \\
0.00000 E+00 \\
0.00000 E+00 \\
0.00000 E+00 \\
2.12676 E+00 \\
1.62595 E+00 \\
5.14129 E-01 \\
1.97217 E-01 \\
0.00000 E+00 \\
0.00000 E+00 \\
0.00000 E+00 \\
0.00000 E+00 \\
0.00000 E+00 \\
0.00000 E+00\end{array}$ & $\begin{array}{l}2.72433 E-01 \\
3.13406 E-01 \\
1.48215 E-01 \\
3.14410 E-01 \\
7.65443 E-03 \\
1.04575 E-02 \\
2.41682 E-02 \\
7.83065 E-03 \\
1.62596 E-01 \\
1.74076 E-01 \\
2.36905 E-01 \\
6.80213 E-01 \\
1.17623 E-00 \\
2.60103 E-01 \\
5.83262 E-02 \\
9.82072 E-04 \\
2.15711 E-03 \\
1.3455 F-03\end{array}$ & 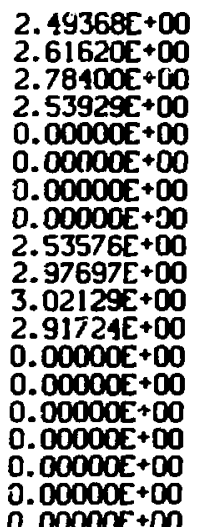 & $\begin{array}{l}+\infty \\
+\infty 0 \\
+\infty \\
+\infty \\
+\infty 0 \\
+\infty 0 \\
+\infty 0 \\
+\infty \\
+\infty \\
+\infty \\
+\infty \\
+\infty \\
+\infty \\
+\infty \\
+\infty \\
+\infty \\
+\infty \\
+\infty\end{array}$ \\
\hline
\end{tabular}


TABLE B-XC

ZPR-3-12 CENTRAL AYEKAGE CROSS SECTIONS

\begin{tabular}{|c|c|}
\hline Holerigh & Fistion. \\
\hline $\begin{array}{l}U-235 \\
U-238 \\
U-234 \\
C \\
F E \\
C R \\
N I \\
N N-55 \\
S I \\
U-233 \\
P U-239 \\
P U-240 \\
T A-181 \\
N B-93 \\
M O \\
A L-27\end{array}$ & $\begin{array}{l}1.47080 E+00 \\
7.54600 E-02 \\
5.23721 E-01 \\
0.00000 E+00 \\
0.00000 E+00 \\
0.00000 E+00 \\
0.00000 E+00 \\
0.00000 E+00 \\
0.00000 E+00 \\
2.23671 E+00 \\
1.66850 E+00 \\
5.64813 E-01 \\
0.00000 E+00 \\
0.00000 E+00 \\
0.00000 E+00 \\
0.00000 E+00\end{array}$ \\
\hline
\end{tabular}

\begin{tabular}{l} 
Copture \\
\hline $3.24017 \mathrm{E}-01$ \\
$1.74709 \mathrm{E}-01$ \\
$3.61285 \mathrm{E}-01$ \\
$7.63476 \mathrm{E}-04$ \\
B.13856E-03 \\
$1.31696 \mathrm{E}-02$ \\
$3.11305 \mathrm{E}-02$ \\
$1.2294 \mathrm{E}-02$ \\
$4.28861 \mathrm{E}-03$ \\
$1.75957 \mathrm{E}-01$ \\
$2.29775 \mathrm{E}-01$ \\
$2.92794 \mathrm{E}-01$ \\
$3.55591 \mathrm{E}-01$ \\
$1.01889 \mathrm{E}-01$ \\
$7.42952 \mathrm{E}-02$ \\
$2.70030 \mathrm{E}-03$
\end{tabular}

\begin{tabular}{c}
$M U$ \\
\hline $2.49667 E+00$ \\
$2.77751 E+00$ \\
$2.56612 E+00$ \\
$0.00000++\infty 0$ \\
$0.00000 E+\infty 0$ \\
$0.00000 E+\infty 0$ \\
$0.00000 E+\infty 0$ \\
$0.00000 E+\infty 0$ \\
$0.00000 E+00$ \\
$2.51259 E+00$ \\
$2.98820 E+00$ \\
$3.04740 E+00$ \\
$0.00000 E+00$ \\
$0.00000 E+00$ \\
$0.00000 E+00$ \\
$0.00000 E+00$
\end{tabular}

Ironsport

$7.06850 E+00$

$7.06842 E+00$

$7.89222 \tau+00$

$2.91862 \mathrm{E}+00$

$3.10207 \mathrm{E}+\infty 0$

$3.49291 \mathrm{E}+00$

$4.89519 E+00$

$4.73035 E+00$

$2.81000 E+C D$

$6.62821 E+\infty 0$

$7.09992 E+00$

$7.05364 E+00$

$5.68181 E+00$

$5.52959 E+00$

$5.91875 E+00$

$3.21665 \bar{C}+00$

TABLE $\mathrm{B}-\mathrm{XC1}$

ZEBRA-2 CENTRAL AVERACE CROSS SECTIONS

\begin{tabular}{|c|c|c|c|c|}
\hline Material & Fiseion & Copture & Mu & Ironsport \\
\hline $\begin{array}{l}U-235 \\
U-238 \\
H-1 \\
0-16 \\
F E \\
C R \\
C U \\
M 0 \\
N N-55 \\
N I \\
\text { RL-27 } \\
T I \\
S I \\
V \\
C \\
U-233 \\
U-234 \\
U-236 \\
P U-239 \\
P U-240 \\
N P-237 \\
A U-197 \\
T A-181 \\
N A-23 \\
B-10 \\
B-11 \\
L I-6 \\
L I-7 \\
\text { HF }\end{array}$ & 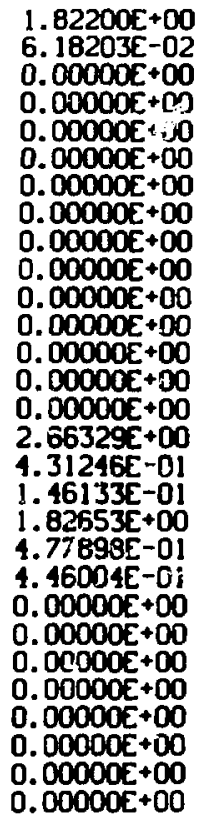 & $\begin{array}{l}5.06840 E-01 \\
2.36585 E-01 \\
1.83367 \mathrm{E}-041 \\
1.40347 \mathrm{E}-03 \\
1.02935 \mathrm{E}-02 \\
1.83360 \mathrm{E}-02 \\
6.06311 \mathrm{E}-02 \\
1.33042 \mathrm{E}-01 \\
5.22785 \mathrm{E}-02 \\
3.27824 \mathrm{E}-02 \\
3.17766 \mathrm{E}-03 \\
1.32061 \mathrm{E}-02 \\
3.96612 \mathrm{E}-03 \\
1.32976 \mathrm{E}-02 \\
6.16632 \mathrm{E}-04 \\
2.50321 \mathrm{E}-01 \\
5.62999 \mathrm{E}-0 \mathrm{1} \\
5.19800 \mathrm{E}-01 \\
4.73230 \mathrm{E}-01 \\
5.12581 \mathrm{E}-01 \\
1.52683 \mathrm{E}+00 \\
5.81715 \mathrm{E}-01 \\
7.77696 \mathrm{E}-01 \\
3.02310 \mathrm{E}-03 \\
2.42691 \mathrm{E}+\mathrm{CO} \\
1.22183 \mathrm{E}-04 \\
9.94745-01 \\
1.12518 \mathrm{E}-05 \\
6.66588 \mathrm{E}-01\end{array}$ & 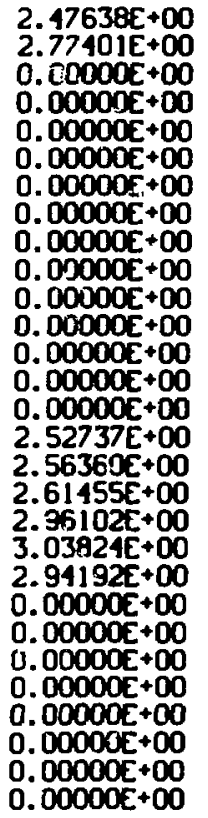 & 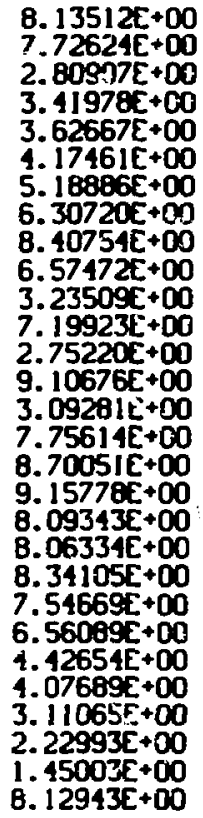 \\
\hline
\end{tabular}




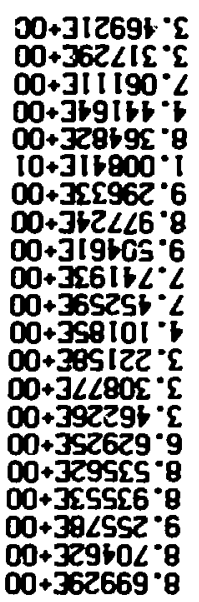

TJodswoJ
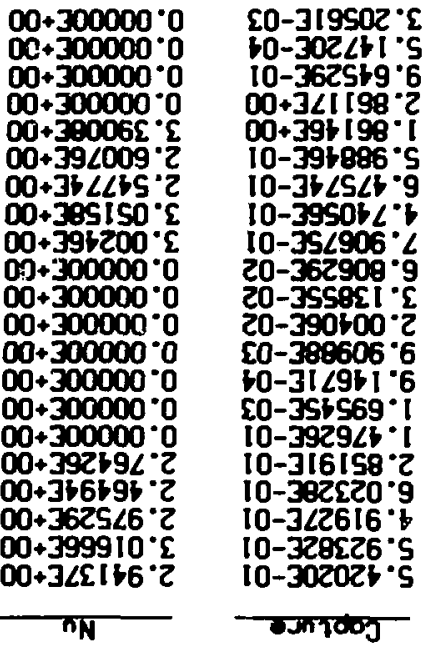

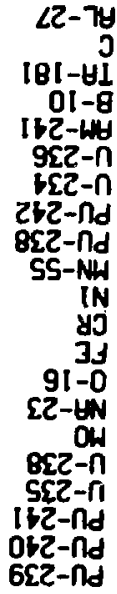

Wo'?

$00+300000^{\circ} 0$

$00+300000^{\circ} 0$

$00+300000^{\circ} 0$

$00+300000^{\circ} 0$

$\left[0-3 \Sigma \angle 680^{\circ} \varepsilon\right.$

10-319660 I

10-356196' 1

$10-30 \angle 4 S \angle L^{\circ} 2$

$00+360551 \cdot 1$

$00+300000^{\circ} 0$

$00+300000 \cdot 0$

$00+300000^{\circ} 0$

$00+300000$ ' 0

$00+300000^{\circ} 0$

$00+300000 \cdot 0$

$00+300000$

20-3SLEOS'

$00+361<\angle 6{ }^{\circ} 1$

$00+36286 S^{\circ} \mathrm{C}$

$10-366288^{\circ} \varepsilon$

$00+39 I 5 S 8$ I

Jo? Jintop

\section{SNOILJJS SSOYJ 30483A甘 7H\&LN3J L-9-8dZ}

\section{I IJX-8 3784I}

\begin{abstract}
$00+3 \angle 69 Z \varepsilon^{\cdot} \cdot 9$ $00+328568 \cdot 8$ $00+361666 \cdot 6$ $00+3$ ESTE6 8 $00+3027 \angle \angle{ }^{\circ} 8$ $00+3 \angle S 26 \angle{ }^{\circ} \cdot$ $00+305966^{\circ}<$ $10+309590 * 1$ $00+32251 \angle{ }^{\circ} 6$ $00+32 \angle 620 \cdot 6$ $00+31 \varepsilon \angle 6 \varepsilon \cdot \varepsilon$ $00+30616<\cdot 2$ $00+3 \angle 8138 \cdot 9$ $00+318061 \cdot 9$ $00+362618.8$ $00+362891 \cdot 8$ $00+36161 \varepsilon: 8$ $00+36200 \varepsilon \cdot \varepsilon$ $00+366915 \cdot \varepsilon$ $00+390919 \cdot \varepsilon$ $00+352 Z 06-\varepsilon$ $00+358 \angle 2\}-\varepsilon$ $00+366068 \cdot 8$ $00+359998-6$ $00+386868^{\circ} 8$ $10+302220 \cdot 1$ $00+366896-6$ $00+319908^{\circ} 6$ $00+3516 \angle 1-6$ $00+321091^{\circ} 6$
\end{abstract}

Troow00J

\begin{abstract}
$00+300000^{\circ} 0$ $00+300000 * 0$ $00+300000^{\circ} 0$ $00+300000 \cdot 0$ $00+300000^{\circ} 0$ $00+300000^{\circ} 0$ $00+300000^{\circ} 0$ $00+3$ LC66S $^{\circ} \mathrm{C}$ $00+39996 S^{-} \cdot 2$ $00+368915^{\circ} 2$ $00+300000^{\circ} 0$ $00+300000^{\circ} 0$ $00+300000^{\circ} 0$ $00+300000^{\circ} 0$ $00+300000=0$ $00+300000^{\circ} 0$ $00+300000$ $00+300000^{\circ} 0$ $00+300000^{\circ} 0$ $00+300000^{\circ} 0$ $00+300000^{\circ} 0$ $00+300000=0$ $00+329 \varepsilon 9 L^{\circ} \mathrm{Z}$ $00+3$ St98 -2 $00+35988 \varepsilon^{\circ} \varepsilon$ $00+322100^{\circ} \varepsilon$ $00+306050^{-\varepsilon} \varepsilon$ $00+398 b \angle 6^{\circ} \mathrm{C}$ $00+3$ K2S10 $\varepsilon$ $00+35 S_{06} 6^{\circ} \mathrm{C}$
\end{abstract}

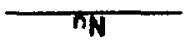

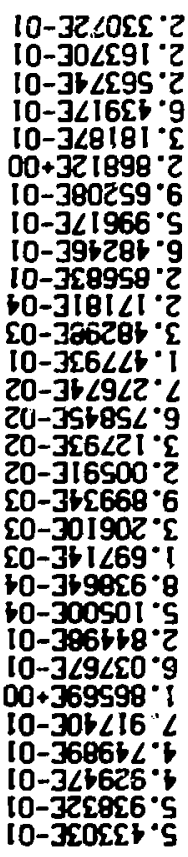

singor

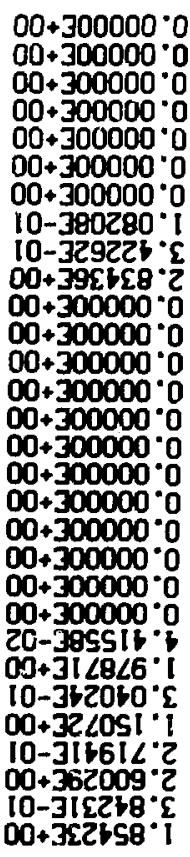

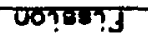

E6-8N 98I-M เ8I-M E8I-M Z8I-M $01-8$ $|B|-H I$ 9६2-ก เEZ-ก EEZ-ก I-H IS OW กอ SS-NH IN d) <2- ไป 乏Z- UN 9I-0

8c2-ก SE2-n Ib2-1uy Bez-nd ZbZ-nd It 2 -nd obz-nd 6Ez-nd Tण!JरणW

SNOLIJ35 SSO\&D 39643AH THIN3J Z-8ddZ

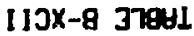


TABLE B-XCIV

2PR-3-568 CENTRAL AVERREE CROSS SECTIONS

\begin{tabular}{|c|c|c|c|c|}
\hline Molerial & Fistion & Cooture & Ev & Ironeporl \\
\hline $\begin{array}{l}U-235 \\
U-238 \\
P U-239 \\
P U-240 \\
0-16 \\
C \\
C A-23 \\
C R \\
F E \\
N I \\
M 0 \\
M-55 \\
U-233 \\
U-234 \\
U-236 \\
B-10 \\
T A-181\end{array}$ & 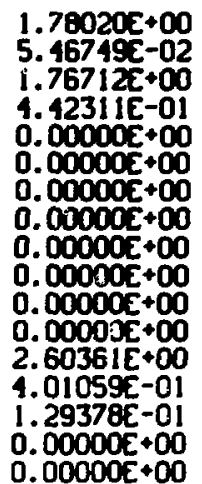 & $\begin{array}{l}4.97516 E-01 \\
2.16260 E-01 \\
4.17264 E-01 \\
4.74509-01 \\
1.14367 E-03 \\
5.90356 E-04 \\
1.50519 E-03 \\
378634 E-02 \\
9.01172 z-03 \\
3.13030 E-02 \\
1.17822 E-01 \\
4.26558 E-02 \\
2.45225 E-01 \\
5.32939 E-01 \\
5.02209 E-01 \\
2.33732 E+00 \\
7.18004 E-01\end{array}$ & 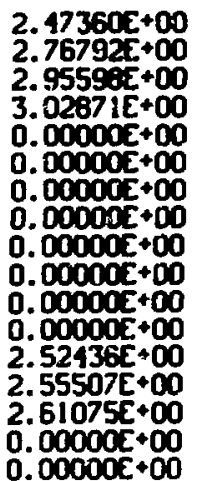 & 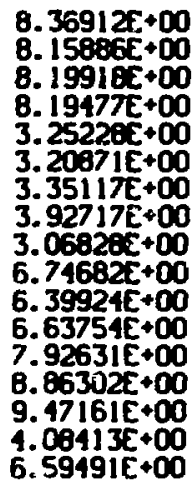 \\
\hline
\end{tabular}

TRBLE B-XCV

2PR-6-6A CENTRAL AVERPEE CPOSS SECTIONS

\begin{tabular}{|c|c|}
\hline aterial & Fingion \\
\hline $\begin{array}{l}U-235 \\
U-235 \\
M A-23 \\
0-16 \\
F E \\
N I \\
C R \\
M-55 \\
U-234 \\
U-236 \\
M O \\
P U-239 \\
B-10 \\
T A-181 \\
C\end{array}$ & 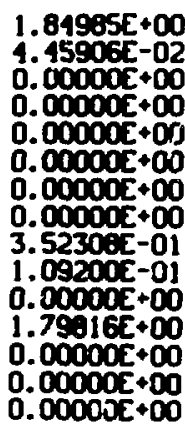 \\
\hline
\end{tabular}

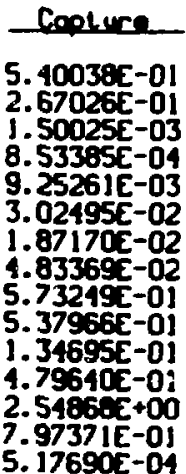

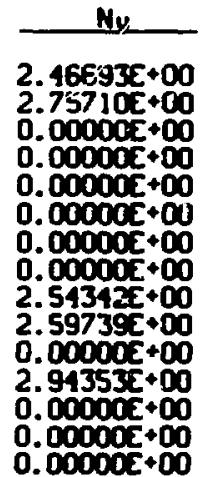

Ironspore

8. 72841E+CO

0. $467695+00$

$3.42697 \mathrm{D}+00$

$3.322055+00$

$3.14317 \mathrm{E}+00$

$7.175025+00$

$4.02193 \mathrm{0}+00$

$7.14602 \mathrm{I}+00$

9. $15936 \pi+\infty 0$

$9.8919 .5+00$

$6.70358 \div+00$

$8.57957 E+00$

$4.28114 E+\infty 0$

$6.855365+00$

3. $30644 E+\infty 0$ 


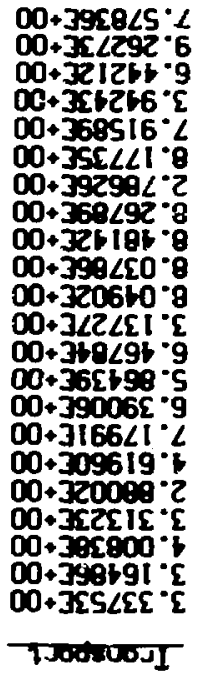

$00+3 \angle+15+<$ 00 306511. 6 $00+366602 \cdot 9$ $00+39562 \mathrm{~B}-\varepsilon$ $00+3$ B6ELS ${ }^{\circ} \mathrm{L}$ $00+35958 L \cdot L$ $00+30$ sSS9. 2 $00+311908^{\circ} \mathrm{L}$ $00+3 \angle 2180 \cdot 8$ $00+311999^{\circ} \mathrm{L}$ $00+3 \angle 50 \angle 9^{\circ} \angle$ $00+318820^{\circ} \mathrm{E}$ $00+3 \angle 8 Z 50 \cdot 9$ $00+39$ ISS $^{\circ} \mathrm{s}$ $00+3<6910.9$ $00+36969 L^{\circ} L$ $00+369<9 z-\varepsilon$ $00+3 x=1 \varepsilon 6-\varepsilon$ $00+3 \angle 2+56^{\circ} 2$ $00+392660 \cdot \varepsilon$

TJodud

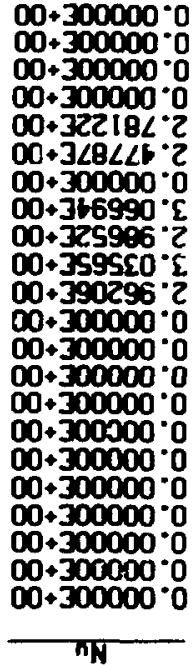

00+3bE89L: $00+3 \angle 266<\cdot 2$ $10-30980<-5$ $00+3528 \angle 0^{\circ} 2$ $10-3$ SESS2 2 I0-39S2E! 20-359956 ' $10-326 b b b^{\circ} \varepsilon$

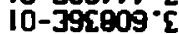
$10-\left.31629\right|^{\circ}$ 10-3I953s $\varepsilon$ EO-3 $\angle 1,05 \cdot 1$ $20-304961^{\circ} \mathrm{c}$ $10-3669 \angle 5 \cdot 1$ $10-3682 \angle 0^{\circ} 1$ $20-3526+9^{\circ} 2$ ع0-3EQSS $\cdot 1$ to-3itios i ع0-39LC96. $20-359699^{\circ} 1$ 60-32SSL.' 9

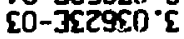

$00+300000 \times 0$ 00.300000 $00+300000^{\circ} 0$ $00+300000^{\circ} 0$ 20-3IStIL'S $00+3196 \angle 9 \cdot 1$ $00+300000^{\circ} 0$ $10-36 \angle 982^{\circ} \varepsilon$ $00+322612 \cdot 2$ 10-3962 IS" $00+3051 \mathrm{~L} \cdot \mathrm{I}$ $00+300000^{-} 0$ $00+300000 * 0$ $00+300000^{\circ} 0$ $00+300000^{\circ} 0$ $00+300000 * 0$ $00+300000 * 0$ $00+300000$ $00+300000 * 0$ $00+300000-0$ $00+300000 * 0$ $00+300000=0$

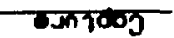

ESI - n3

ISI-ก3

IB! -4$]$
$0 !-8$

BE2-ก

S\&2-ก

2bz-nd

is $2-n$ A

otz-nd

6Ez-nd

9I-0

IN

E6-8N

OW

SS-NW

SW

I-H

3」

ขอ

LZ- W

रण? गुण

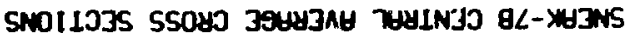

\section{1 $13 x-8$ 3า 941}

\begin{abstract}
$00+300000^{\circ} 0$ $00+300000 \cdot 0$ $00+300000 \cdot 0$ $00+300000 \cdot 0$ $00+3 \angle 2 \angle L L^{\cdot} \cdot 2$ $00+366586 \cdot 2$ $00+300000^{\circ} 0$ $00+3 \angle 1680^{\circ} \varepsilon$ $00+318466 \cdot 2$ $00+325 \angle S 0^{-} \varepsilon$ $00+312 \angle \angle 6^{\circ} \cdot 2$ $00+300000^{\circ} 0$ $00+300000 \cdot 0$ $00+300000 \cdot 0$ $00+300000^{-0}$ $00+300000^{\circ} 0$ $00+300000^{\circ} 0$ $00+300000$ $00+300000^{\circ} 0$ $00+300000^{\circ} 0$
\end{abstract}

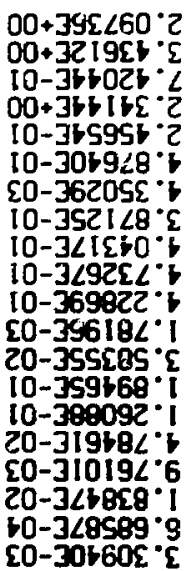

$00+300000 \cdot 0$ $00+300000 \cdot 0$ $00+300000^{\circ} 0$ $00+300000^{\circ} 0$ $20-3106 \angle 8^{\circ} \mathrm{L}$ $00+31<008 \cdot 1$ $00+300000 * 0$ $10-399060^{\circ}$ $00+3 \angle S 69 \varepsilon^{\circ} ?$ I0-3[9608. 5 $00+3$ เ $66 \angle$ - I $00+300000 \cdot 0$ $00+300000^{\circ} 0$ $00+300000^{\circ} 0$ $00+300000 \cdot 0$ $00+300000 \cdot 0$ $00+300000^{\circ} 0$ $00+300000^{\circ} 0$ $00+300000 \cdot 0$ $00+300000^{\circ} 0$

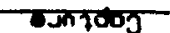

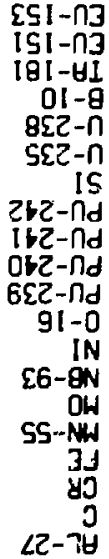

जo?

गणगमण

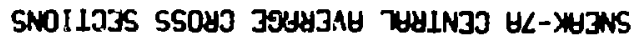

I ควX-8 3า 


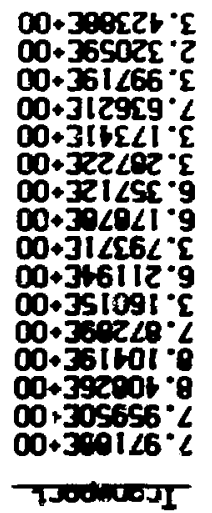

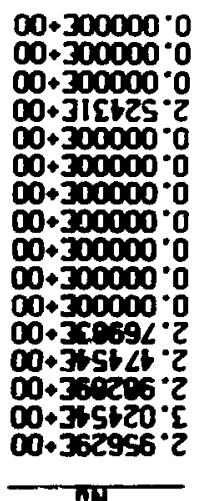

$\mathbf{2 0}-39 \angle \mathbf{5 6} \cdot 2$ $10-305199^{\circ} 6$ $00+3 \angle 0061^{\circ} 2$ I0-360b5E $\cdot 2$ t0-302911' 9 SO-35LOZ6: 1 $10-350.201 \cdot 1$ 20-303159. $\mathrm{C}$ $20-30+069^{\circ} 1$ $20-3$ IE $100^{-\varepsilon}$ 50-345600:6 $10-30$ - $052 \cdot 2$ $10-316269^{\circ}$ 10-300218. io-300<s -1 10-36IBss. 2

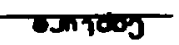

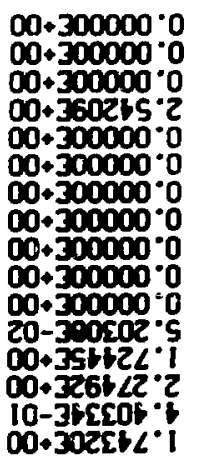

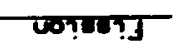

L2- X $9-17$ ot $-\boldsymbol{g}$ Eะट-ก [2SS-MH $\boldsymbol{y}$ IN

$3 \mathrm{~s}$ oxz-n SEZ-ก Itz-nd obz-nd 6ez-nd

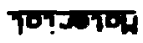

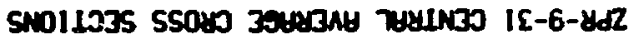

I เ เ AวX-8 3า9y1 